\title{
Aufbau einer \\ Pulslaserdepositions-(PLD)-anlage und \\ Untersuchungen zur PLD in den \\ MAX-Phasen-Systemen Ti-Si-C, Cr-Al-C und Ti-Al-N
}

\author{
Dissertation \\ zur Erlangung des Doktorgrades \\ der Mathematisch-Naturwissenschaftlichen Fakultäten \\ der Georg-August-Universität zu Göttingen
}

vorgelegt von

Christian Lange

aus Dresden

Göttingen 2009 
D 7

Referent:

Korreferent:
Prof. Dr. Peter Schaaf

Prof. Dr. Hans-Ulrich Krebs

Tag der mündlichen Prüfung: 12.06.2009 
Den Menschen dieser Stadt, insbesondere Mara Kastein.

Bien sûr, ce n'est pas la Seine, Ce n'est pas le bois de Vincennes, Mais c'est bien joli tout de même,

A Göttingen, à Göttingen.

Barbara, "Göttingen" (Editions Métropolitaines, 1969) 



\section{Inhaltsverzeichnis}

1 Einleitung 1

2 Grundlagen 3

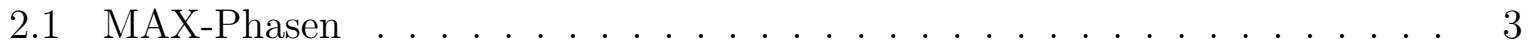

2.1 .1 Allgemeines . . . . . . . . . . . . . . . . . . 3

2.1.2 Das System Ti- Si - C . . . . . . . . . . . . . 9

2.1.3 Das System Cr - Al - C . . . . . . . . . . . . . . . . . 14

2.1.4 Das System Ti - Al - N . . . . . . . . . . . . . . . 17

2.2 Pulslaserdeposition (Pulsed Laser Deposition) . . . . . . . . . . . . . . . . 22

2.2.1 Experimentelle Grundlagen . . . . . . . . . . . . . . . . . . . . 22

2.2.2 Eigenschaften des PLD-Plasmas . . . . . . . . . . . . . . 27

2.2 .3 Reaktive PLD . . . . . . . . . . . . . . . . . . . . . 28

2.2.4 Ionenstrahlunterstützte PLD . . . . . . . . . . . . . . 31

3 Methoden $\quad 36$

3.1 Röntgendiffraktometrie . . . . . . . . . . . . . . . . 36

3.2 Glimmentladungsemissionsspektroskopie . . . . . . . . . . . . . . 38

3.3 Röntgen-Photoelektronenspektroskopie . . . . . . . . . . . . . . . . 43

3.4 Mikrohärtemessungen . . . . . . . . . . . . . . . . 45

3.5 Elektronenmikroskopie . . . . . . . . . . . . . . . . 46

3.5.1 Rasterelektronenmikroskopie . . . . . . . . . . . . . . . . . 46

3.5.2 Transmissionselektronenmikroskopie . . . . . . . . . . . . . . 46

3.6 Messung elektrischer Eigenschaften . . . . . . . . . . . . . . . . 47

4 Aufbau der PLD-Anlage $\quad 49$

4.1 Rohbau . . . . . . . . . . . . . . . . . . . . . . . . 49

4.2 Substratheizung . . . . . . . . . . . . . . . . . . . 52

4.3 Ionenquelle . . . . . . . . . . . . . . . . . . 54

4.4 Vakuumpumpen . . . . . . . . . . . . . . . . . . . 55

4.5 Targethalter . . . . . . . . . . . . . . . . . 57

4.6 Laser . . . . . . . . . . . . . . . . . . . . . . . . . . 59 
5 Herstellung der Proben $\quad 62$

6 Ergebnisse der Deposition von MAX-Phasen-Targets 66

$6.1 \mathrm{Ti}_{3} \mathrm{SiC}_{2}$-Target . . . . . . . . . . . . . . . . . 66

6.1.1 $\mathrm{MgO}(100)-$ Substrat . . . . . . . . . . . . . . . 66

6.1.1.1 GDOES-Messungen . . . . . . . . . . 66

6.1.1.2 Röntgendiffraktometrie und TEM-Messungen . . . . . . . 70

6.1.1.3 Härtemessungen . . . . . . . . . . . . . . . . . . 75

$6.1 .2 \mathrm{Si}(100)-$ Substrat . . . . . . . . . . . . . . . . . 78

6.1.2.1 GDOES-Messungen . . . . . . . . . . . 78

6.1.2.2 Röntgendiffraktometrie . . . . . . . . . . . . 78

6.1.2.3 Härtemessungen . . . . . . . . . . . . . . . . 80

6.1.2.4 XPS-Messungen .................. . . 82

6.1.3 Elektrische Eigenschaften . . . . . . . . . . . . . . 85

6.1.4 Edelstahlsubstrat . . . . . . . . . . . . . . . . . 86

6.1.4.1 GDOES-Messungen . . . . . . . . . . 87

6.1.4.2 Röntgendiffraktometrie . . . . . . . . . . . . . . 89

6.1.4.3 Härtemessungen . . . . . . . . . . . . . . . . . . . 91

6.1.5 Zusammenfassung - $\mathrm{Ti}_{3} \mathrm{SiC}_{2}$-System . . . . . . . . . . . . . . 92

$6.2 \mathrm{Cr}_{2}$ AlC-Target . . . . . . . . . . . . . . . 96

6.2.1 $\mathrm{MgO}(100)-$ Substrat . . . . . . . . . . . . . . . 96

6.2.1.1 GDOES-Messungen . . . . . . . . . . . . . 96

6.2.1.2 Röntgendiffraktometrie und TEM-Messungen . . . . . . . 100

6.2.1.3 Härtemessungen . . . . . . . . . . . . . . . . . 104

$6.2 .2 \quad \mathrm{SiO}_{2}$-Substrat . . . . . . . . . . . . . . . . . 105

6.2.2.1 GDOES-Messungen . . . . . . . . . . . 105

6.2.2.2 Röntgendiffraktometrie . . . . . . . . . . . . . 107

6.2.2.3 Härtemessungen . . . . . . . . . . . . . . . . . . . . . . . 109

6.2.3 Elektrische Eigenschaften . . . . . . . . . . . . . . . . . . 110

6.2.4 Edelstahlsubstrat . . . . . . . . . . . . . . . . . 111

6.2.4.1 GDOES-Messungen . . . . . . . . . . . . 111

6.2.4.2 Röntgendiffraktometrie . . . . . . . . . . . . . . 113

6.2.4.3 Härtemessungen . . . . . . . . . . . . . . . 116

6.2.5 Zusammenfassung $-\mathrm{Cr}_{2}$ AlC-System . . . . . . . . . . . . 117

7 Ergebnisse der reaktiven PLD $\quad 119$

7.1 GDOES-Ergebnisse . . . . . . . . . . . . . . . 120

7.1.1 Mittlere Elementkonzentrationen $C_{i} \ldots \ldots$. . . . . . . 120

7.1 .2 Elementprofile . . . . . . . . . . . . . . . . . . . . 121 
8 Zusammenfassung und Ausblick $\quad 126$

$\begin{array}{lr}\text { Abbildungsverzeichnis } & 129\end{array}$

$\begin{array}{ll}\text { Tabellenverzeichnis } & 133\end{array}$

$\begin{array}{ll}\text { Literaturverzeichnis } & 134\end{array}$ 



\section{Einleitung}

Sowohl in der naturwissenschaftlichen Forschung als auch bei industriellen Anwendungen spielt die Materialklasse der Keramiken ein wichtige Rolle. Die Bezeichnung Keramik stammt vom altgriechischen $\kappa \epsilon \rho \alpha \mu \iota \kappa o ́ \varsigma$ (keramikos), das so viel wie Töpferware bedeutet, ab. Heutzutage versteht man unter Keramiken anorganische, nichtmetallische Verbindungen, die bei hohen Temperaturen synthetisiert werden. Dazu gehören im Wesentlichen Oxide, Nitride, Carbide, Boride, Silicide und Sulfide.

Keramiken können grob in zwei Gruppen eingeteilt werden. Zum einen sind dies traditionelle Keramiken wie Ziegel, Kacheln, Porzellan und Steinzeug. Die andere Gruppe umfasst moderne Funktionskeramiken. Durch angepasste chemische Zusammensetzungen werden diesen Funktionskeramiken besondere Eigenschaften verliehen, die sie für technische Anwendungen sehr wertvoll machen. $\mathrm{Zu}$ diesen besonderen Eigenschaften und ihren Anwendungen gehören unter anderem die besonders große Härte von Siliziumcarbid ( $\mathrm{SiC}$ ), Wolframcarbid (WC) oder Titannitrid (TiN) in Beschichtungen für Schneidwerkzeuge oder in Verschleißschutzschichten, die Piezoelektrizität von Bariumtitanat $\left(\mathrm{BaTiO}_{3}\right)$ in $\mathrm{Mi}$ kroaktuatoren oder Ultraschallanwendungen, die Sauerstoffionenleitfähigkeit von ZirkonMetalloxid-Mischkeramiken $\left(\mathrm{ZrO}_{2}-\mathrm{M}_{2} \mathrm{O}_{3}, \mathrm{M}=\mathrm{Yb}, \mathrm{Gd}, \mathrm{Nd}, \mathrm{Y}\right)$ in Festoxidbrennstoffzellen und Sauerstoffsensoren ( $\lambda$-Sonden), Supraleitung in zum Beispiel Y-Ba-Cu-O oder Bi-Sr-Cu-O-Keramiken und die stimulierte Strahlungsemission in dotierten $\mathrm{Y}_{3} \mathrm{Al}_{5} \mathrm{O}_{12^{-}}$ Granatkeramiken (Nd:YAG-Laser). Eine ausführliche Übersicht zu modernen Funktionskeramiken findet sich in [1].

Eine relativ neue Klasse von Funktionskeramiken stellen die so genannten MAX-Phasen dar. Bei MAX-Phasen handelt es sich um ternäre Carbid- bzw. Nitridverbindungen der chemischen Zusammensetzung $\mathrm{M}_{n+1} \mathrm{AX}_{n}$, wobei $\mathrm{M}$ ein Übergangsmetall der Gruppen 3 bis 6, A ein Hauptgruppenelement der Gruppen 13 bis 16, sowie X Kohlenstoff bzw. Stickstoff sind. MAX-Phasen vereinen sowohl keramische als auch metallische Eigenschaften in sich. Wie Keramiken verfügen sie über eine hohe thermische Stabilität und Oxidationsbeständigkeit bei hohen Temperaturen. Gleichzeitig sind sie wie Metalle relativ gute elektrische und thermische Leiter, sowie resistent gegen thermische Schockbelastungen. Aufgrund ihrer besonderen nano-laminaren Kristallstruktur sind sie außerdem sehr gut spanend zu bearbeiten und verfügen über einen außergewöhnlich hohe Toleranz gegenüber mechanischer Belastung. Diese Eigenschaften ermöglichen eine Anwendung von 
MAX-Phasen als elektrisch leitende Verschleißschutzschichten.

Zur Deposition von dünnen Schichten existiert mittlerweile eine Vielzahl von experimentellen Techniken. Zu den wichtigsten gehören hierbei thermisches Verdampfen, chemische Gasphasenabscheidung, Magnetronsputtern und die Pulslaserdeposition (PLD). Die Vorteile der PLD liegen im relativ einfachen experimentellen Aufbau und der Möglichkeit -bei entsprechender Laserleistung- jedes Material in die Gasphase zu überführen. Aufgrund der schnellen Prozessführung außerhalb eines Gleichgewichtes ist diese Überführung in die Gasphase weitestgehend kongruent, das heißt dass die Elementzusammensetzung des Targets zunächst im Plasma erhalten wird.

Die Ziele dieser Arbeit bestehen im Aufbau einer solchen Anlage zur Pulslaserdeposition, sowie in Untersuchungen zur PLD in den drei MAX-Phasen-relevanten Elementsystemen Ti-Si-C, Cr-Al-C und Ti-Al-N. Die PLD-Anlage verfügt zusätzlich über eine Ionenquelle, mit deren Hilfe ein Ionenstrahl mit Beschleunigungsspannungen von 100-5000 V während der Deposition auf das Substrat gerichtet werden kann. Auf diese Weise wird es möglich, das Schichtwachstum mit einem Beschuss von inerten Argonionen zu beeinflussen, beziehungsweise MAX-Nitride durch Verwendung eines Stickstoff-Ionenstrahls reaktiv herzustellen. Die Untersuchungen in den beiden Carbidsystemen erfolgten durch die PLD von vorsynthetisierten MAX-Phasentargets der Zusammensetzungen $\mathrm{Ti}_{3} \mathrm{SiC}_{2}$ und $\mathrm{Cr}_{2} \mathrm{AlC}$. Zur Herstellung der Nitridphasen wurde die PLD von Titan- und AluminiumElementtargets unter Verwendung des reaktiven Stickstoff-Ionenstrahls durchgeführt.

In Kapitel 2 dieser Arbeit werden die theoretischen Grundlagen zu MAX-Phasen und der Pulslaserdeposition näher erläutert. Kapitel 3 beschreibt die im Rahmen dieser Arbeit verwendeten Charakterisierungsmethoden. Eine detaillierte Zusammenfassung des Aufbaus der PLD-Anlage ist in Kapitel 4 dargestellt. Die Herstellung der Proben wird in Kapitel 5 erklärt. Die experimentellen Ergebnisse und ihre Diskussion finden sich in den Kapiteln 6 ( $\mathrm{Ti}_{3} \mathrm{SiC}_{2^{-}}$und $\mathrm{Cr}_{2} \mathrm{AlC}$-Target) und 7 (reaktive PLD von Ti/Al). Diese Arbeit stellt den Anfang der Arbeiten an einem DFG-Projekt zur PLD von MAX-Phasen dar. Aus diesem Grund liefern die Ergebnisse zunächst einen groben Überblick über grundsätzliche Zusammenhänge bei der Verwendung der hier neu aufgebauten Anlage. Eine Zusammenfassung der Arbeit und ein Ausblick auf zukünftig notwendige Arbeiten gibt das Kapitel 8. 


\section{Grundlagen}

\subsection{MAX-Phasen}

\subsubsection{Allgemeines}

Als MAX-Phasen werden ternäre Carbid- bzw. Nitridverbindungen der Zusammensetzung $\mathrm{M}_{n+1} \mathrm{AX}_{n}$ mit $\mathrm{M}=$ Übergangsmetall der Gruppen 3 bis $6, \mathrm{~A}=$ Hauptgruppenelement der Gruppen 13 bis 16 und $\mathrm{X}=\mathrm{C}$ bzw. $\mathrm{N}$ bezeichnet [2]. MAX-Phasen kristallisieren in einer hexagonalen Struktur $\left(P 6_{3} / m m c\right)$. In ihr bilden die Elemente M und X Schichten aus kantenverknüpften $\mathrm{M}_{6} \mathrm{X}$-Oktaedern, zwischen denen einzelatomare Schichten des AElements eingelagert sind. Die kovalenten Bindungen in den $\mathrm{M}_{6} \mathrm{X}$-Oktaedern sind dabei deutlich stärker als die Bindungskräfte zwischen dem M-Metall der Oktaeder und dem A-Metall in den interkalierten Schichten. Auf diese Weise entsteht eine nano-laminare Struktur mit anisotropen Eigenschaften. Diese Schichtstruktur und ihr Verhalten unter Scher- bzw. Druckbelastung sind der Grund für die bemerkenswerten mechanischen Eigenschaften der MAX-Phasen [3]. Bisher wurden über $50 \mathrm{M}_{n+1} \mathrm{AX}_{n}$-Verbindungen für $\mathrm{n}=1,2,3-\operatorname{mit} M=\{\mathrm{Sc}, \mathrm{Ti}, \mathrm{V}, \mathrm{Cr}, \mathrm{Zr}, \mathrm{Nb}, \mathrm{Mo}, \mathrm{Hf}, \mathrm{Ta}\}, A=\{\mathrm{Al}, \mathrm{Si}, \mathrm{P}, \mathrm{S}, \mathrm{Ga}$, Ge, As, Cd, In, Sn, Tl, Pb $\}$ und $X=\mathrm{C}$ oder N synthetisiert und beschrieben. Aufgrund der Vielzahl der Veröffentlichungen zu diesem Thema sei hier auf einen ausführlichen Übersichtsartikel verwiesen [4]. Ausgehend von der Zahl der M-, A- und X-Elemente im Molekül, entsprechend $n=1,2$, oder 3, ergeben sich drei Gruppen von MAX-Phasen, die als 211, 312 und 413 bezeichnet werden. Diese Gruppierung ist in Abb. 2.1 mit den bisher synthetisierten MAX-Phasen aufgeführt.

Trotz der unterschiedlichen stöchiometrischen Zusammensetzung bleibt die nano-laminare Kristallstruktur in allen drei Gruppen erhalten. Lediglich die Stapelfolge von $\mathrm{M}_{6} \mathrm{X}$-Oktaedern und A-Zwischenschichten ändert sich. In den 211-Phasen gibt es zwei aufeinander folgende M-Atomlagen, in den 311-Phasen entsprechend drei und in den 413-Phasen vier M-Atomlagen, die durch eine monoatomare Schicht des A-Elementes getrennt werden. Die Illustration in Abbildung 2.2 verdeutlich dieses.

Die ursprüngliche Synthese und die erste kristallographische Charakterisierung von MAXPhasen erfolgten bereits in den 1960er Jahren durch Nowotny et al. [5-9]. Allerdings erst 


\section{1}

\begin{tabular}{|c|c|c|c|c|c|}
\hline $\mathrm{Ti}_{2} \mathrm{AlC}^{*}$ & $\mathrm{Ti}_{2} \mathrm{AIN}^{*}$ & $\mathrm{Hf}_{2} \mathrm{PbC}^{*}$ & $\mathrm{Cr}_{2} \mathrm{GaC}$ & $\mathrm{V}_{2} \mathrm{AsC}$ & $\mathrm{Ti}_{2} \operatorname{lnN}$ \\
\hline $\mathrm{Nb}_{2} \mathrm{AlC}^{*}$ & $(\mathrm{Nb}, \mathrm{Ti})_{2} \mathrm{AlC} *$ & $\mathrm{Ti}_{2} \mathrm{AIN}_{0.5} \mathrm{C}_{0.5}{ }^{*}$ & $\mathrm{Nb}_{2} \mathrm{GaC}$ & $\mathrm{Nb}_{2} \mathrm{AsC}$ & $\mathrm{Zr}_{2} \operatorname{lnN}$ \\
\hline $\mathrm{Ti}_{2} \mathrm{GeC}^{*}$ & $\mathrm{Cr}_{2} \mathrm{AlC}$ & $\mathrm{Zr}_{2} \mathrm{SC}$ & $\mathrm{Mo}_{2} \mathrm{GaC}$ & $\mathrm{Ti}_{2} \mathrm{CdC}$ & $\mathrm{Hf}_{2} \operatorname{lnN}$ \\
\hline $\mathrm{Zr}_{2} \mathrm{SnC}^{*}$ & $\mathrm{Ta}_{2} \mathrm{AlC}$ & $\mathrm{Ti}_{2} \mathrm{SC}$ & $\mathrm{Ta}_{2} \mathrm{GaC}^{*}$ & $\mathrm{Sc}_{2} \operatorname{lnC}$ & $\mathrm{Hf}_{2} \mathrm{SnN}$ \\
\hline $\mathrm{Hf}_{2} \mathrm{SnC}^{*}$ & $\mathrm{~V}_{2} \mathrm{AIC}$ & $\mathrm{Nb}_{2} \mathrm{SC}$ & $\mathrm{Ti}_{2} \mathrm{GaN}$ & $\mathrm{Ti}_{2} \ln \mathrm{C}$ & $\mathrm{Ti}_{2} \mathrm{TIC}$ \\
\hline $\mathrm{I}_{2} \mathrm{SnC}^{*}$ & $\mathrm{~V}_{2} \mathrm{PC}$ & $\mathrm{Hf}_{2} \mathrm{SC}$ & $\mathrm{Cr}_{2} \mathrm{GaN}$ & $\mathrm{Zr}_{2} \ln \mathrm{C}$ & $\mathrm{Zr}_{2} \mathrm{TIC}$ \\
\hline $\mathrm{b}_{2} \mathrm{SnC}^{*}$ & $\mathrm{Nb}_{2} \mathrm{PC}$ & $\mathrm{Ti}_{2} \mathrm{GaC}$ & $\mathrm{V}_{2} \mathrm{GaN}$ & $\mathrm{Nb}_{2} \operatorname{lnC}$ & $\mathrm{Hf}_{2} \mathrm{TIC}$ \\
\hline $\mathrm{r}_{2} \mathrm{PbC}^{*}$ & $\mathrm{Ti}_{2} \mathrm{PbC}^{*}$ & $\mathrm{~V}_{2} \mathrm{GaC}$ & $\mathrm{V}_{2} \mathrm{GeC}$ & $\mathrm{Hf}_{2} \operatorname{lnC}$ & $\mathrm{Zr}_{2} \mathrm{TIN}$ \\
\hline
\end{tabular}

\section{2}

$\mathrm{Ti}_{3} \mathrm{AlC}_{2}{ }^{*}$

413

$\mathrm{Ti}_{4} \mathrm{AlN}_{3}{ }^{*}$

Abbildung 2.1: In Abhängigkeit von $n$ ergeben sich für die $\mathrm{M}_{n+1} \mathrm{AX}_{n}$-Phasen drei Gruppen, die als 211, 312 und 413 bezeichnet werden. In diesen Gruppen gibt es mittlerweile zahlreiche Verbindungen, die bisher synthetisiert wurden. [2].
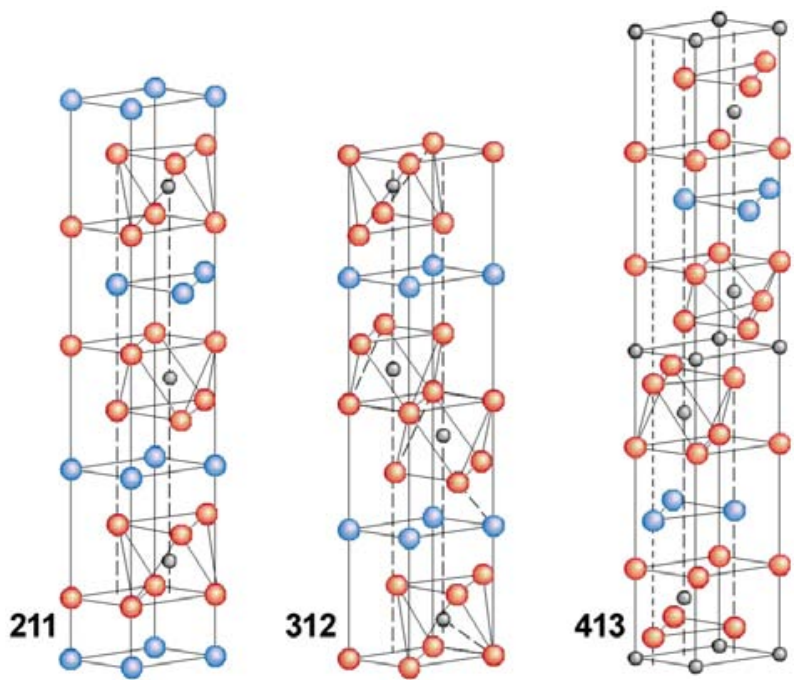

Abbildung 2.2: Aufgrund der unterschiedlichen Zusammensetzung in den 211-, 312- und 413-Gruppen ergeben sich unterschiedliche Stapelfolgen von $\mathrm{M}_{6} \mathrm{X}$-Oktaedern und AElementschichten (rote Atome: M-Element, schwarze Atome: X-Element, blaue Atome: A-Element) [2].

etwa 30 Jahre später wurden die besonderen Eigenschaften der MAX-Phasen -zuerst am Beispiel von $\mathrm{Ti}_{3} \mathrm{SiC}_{2}-$ entdeckt und beschrieben [10].

Da die $\mathrm{M}_{6} \mathrm{X}$-Oktaeder in MAX-Phasen vergleichbar mit der Struktur der entsprechenden binären MX-Carbid- bzw. -Nitridverbindungen sind, besitzen die ternären MAXVerbindungen in vielen Hinsichten den MX-Verbindungen ähnliche Eigenschaften, die aber durch die Zwischenschichten aus reinen A-Element-Atomen zum Teil erheblich verändert werden. Die strukturelle Verwandschaft zwischen den ternären MAX-Phasen und den 
entsprechenden binären MX-Verbindungen ist sehr eng. Ersetzt man in der Verbindung $\mathrm{M}_{n+1} \mathrm{AX}_{n} A$ durch $X$, erhält man eine hochgradig verzwillingte MX-Struktur. Durch eine Rotationsbewegung entsteht danach eine geordnete MX-Phase. Diese Entmischungsund Umwandlungsreaktion (Abbildung 2.3) ist der bevorzugte Zerfallsmechanismus zum Beispiel in $\mathrm{Ti}_{3} \mathrm{SiC}_{2}$ (s. Abschnitt 2.1.2).

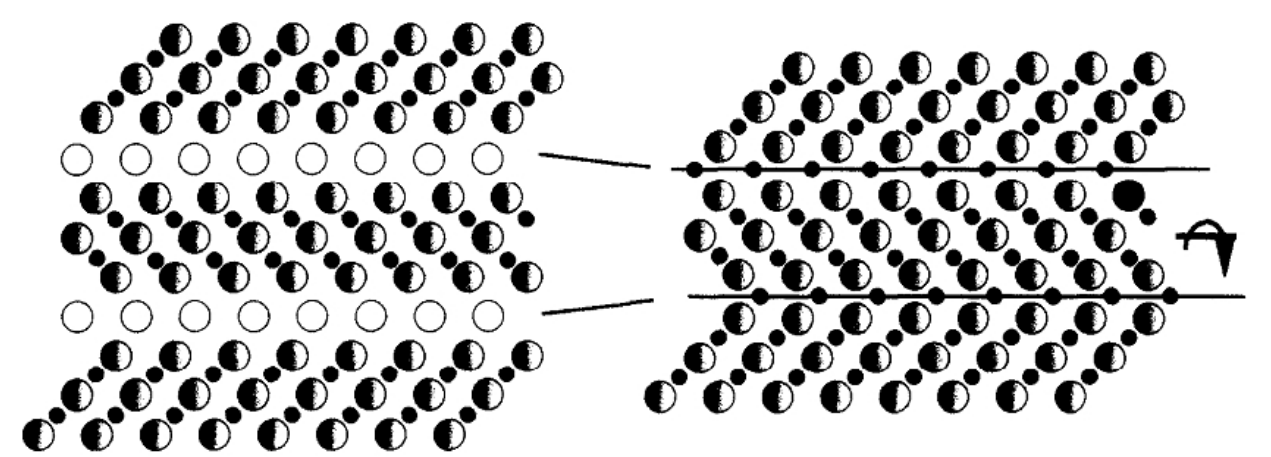

(a)

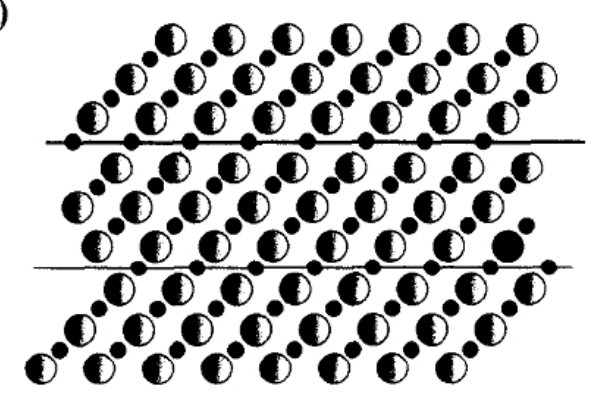

(b)

\footnotetext{
Abbildung 2.3: Durch Ersetzen von $A$ durch $X$ in $\mathrm{M}_{n+1} \mathrm{AX}_{n}$ (a) kommt es zunächst zu Verzwillingungen in der Struktur (b). Diese werden durch eine Rotationsbewegung aufgelöst, und es entsteht die geordnete Struktur der binären MX-Phase (c). (Schwarz-weiß: M-Element, weiß: A-Element, schwarz: x-Element) [4].
}

Des Weiteren ist auffällig, dass ein beinahe 1:1-Verhältnis zwischen dem Gitterparameter $a$ der MAX-Phasen und dem M-M-Abstand in den entsprechenden MX-Verbindungen besteht [11]. Wie bereits erwähnt, sind die Bindungen in den $\mathrm{M}_{6} \mathrm{X}$-Oktaedern sehr viel stärker als die Bindungen zwischen M- und A- Atomen. Die Bindungsstärken zwischen den A-Atomen entlang der monoatomaren Schichten sind noch geringer als die zwischen A- und M-Atomen. Diese -im Vergleich zu MX- veränderte Elektronenverteilung führt zu einer weiteren interessanten Eigenschaft der MAX-Phasen: ihrer vergleichsweise hohen elektrischen Leitfähigkeit. Die Bindung der M-Atome hat in der Nähe der A-Atome einen stärker metallischen Charakter (als in reinen MX-Phasen) und führt damit zu einer erhöhten elektrischen Leitfähigkeit der MAX-Phasen gegenüber den MX-Verbindungen [12, 13]. Tabelle 2.1 zeigt, dass die MAX-Phasen elektrisch vergleichbar gut oder sogar besser als Titan leiten. Bei der Messung der elektrischen Leitfähigkeit von einkristallinen MAX-Proben wurde von Haddad et al. festgestellt, dass die Leitfähigkeit entlang unterschiedlicher Kristallachsen (parallel oder senkrecht zur Schichtstruktur) unterschiedliche 
Tabelle 2.1: Raumtemperaturleitfähigkeiten $\sigma_{300}$ von MAX-Phasen. Die Werte für $\mathrm{TiC}_{x}$ und Ti sind zum Vergleich angegeben.

\begin{tabular}{|c|c|c|}
\hline & $\sigma_{300}\left[(\Omega \cdot \mathrm{m})^{-1}\right]$ & Ref. \\
\hline $\mathrm{Ti}_{3} \mathrm{SiC}_{2}$ & $4,5 \cdot 10^{6}$ & {$[15]$} \\
$\mathrm{Ti}_{3} \mathrm{Al}_{1.1} \mathrm{C}_{1.8}$ & $2,8 \cdot 10^{6}$ & {$[15]$} \\
$\mathrm{TiC}_{0.928}$ & $0,6 \cdot 10^{6}$ & {$[16]$} \\
$\mathrm{TiC}_{>0.969}$ & $1,1 \cdot 10^{6}$ & {$[16]$} \\
$\mathrm{Ti}$ & $2,6 \cdot 10^{6}$ & {$[17]$} \\
\hline
\end{tabular}

Werte annimmt. Allerdings hängt es vom der jeweiligen chemischen Zusammensetzung der MAX-Phasen ab, ob sie parallel oder senkrecht zu den Schichten besser leiten [14].

Verglichen mit den MX-Verbindungen sind MAX-Phasen (mit Härten bis 6 GPa) eher weich [18-20], aber dennoch ziemlich belastungssteif (E-Moduln $E=300-500$ GPa, Schermoduln $G=120-150 \mathrm{GPa}$ ) [21]. Im Gegensatz zu den meisten anderen keramischen Werkstoffen sind MAX-Phasen aber leicht spanend zu bearbeiten und außerdem tolerant gegenüber mechanischer oder thermischer Schockbelastung. Keramische Werkstoffe sind in aller Regel sehr spröde, deswegen schlecht zu bearbeiten und zerspringen bei thermischem Schock - also dem schnellen Abkühlen über große Temperaturbereiche. MAX-Phasen zeigen ein deutlich anderes Verhalten, das durch die nano-laminare Struktur begründet werden kann. Durch diese Schichtstruktur ist es möglich, Schäden in der Kristallstruktur auf einen kleinen Bereich in der Nähe des Schadens zu begrenzen. Risse und Brüche im Material können sich also - im Gegensatz zu normalen Keramiken - nicht weiter vergrößern. Folgende energieabsorbierende Mechanismen spielen dabei ein Rolle: Schichtspaltung und Bruchreflexion, das Ausreißen einzelner Körner und die Stauchung einzelner Körner. Lichtmikroskopische Aufnahmen des Verhaltens der MAX-Nanolaminate sind in Abbildung 2.4 gezeigt. Es ist deutlich erkennbar, welche große Rolle die kristallographische Schichtstruktur spielt. Entlang der Schichten kommt es zu Mikrobrüchen, die ein einfaches Gleiten der Körner gegeneinander ermöglichen. Durch dieses Gleiten und die daraus folgenden Verformungen im Gefüge wird die Energie aus mechanischen Belastungen effizient absorbiert, und die Reichweite von Rissen und Brüchen im Material sehr stark verringert. MAX-Phasen sind deshalb makroskopisch nicht spröde. Den oben genannten Absorptionsmechanismen übergeordnet ist das Entstehen von Knickbändern (engl.: kink bands) in der Gefügestruktur. Aus diesem Grund gehören MAX-Phasen - neben u.a. Graphit, Eis und Glimmer-Schichtsilikaten - in die Gruppe der kinking nonlinear elastic solids [3, 22, 23]. Die Schichtstruktur und das leichte Gleiten entlang der Gitterschichten lassen interessante Eigenschaften bezüglich Reibungskoeefizient und Selbstschmierung bei MAX-Phasen vermuten. Tatsächlich wurden Reibungskraftmessungen am Rasterkraftmikroskop entlang der Basisflächen von $\mathrm{Ti}_{3} \mathrm{SiC}_{2}$ durchgeführt. Diesen ergaben einen Reibungskoeffizienten $\mu$ 
von etwa $2 \cdot 10^{-3}$, also vergleichbar mit den kleinsten Werten, die für Festschmierstoffe wie Graphit und $\mathrm{MoS}_{2}$ gemessen wurden [24, 25]. Diese Werte blieben auch nach einer leichten Zersetzung an Luft über Monate stabil.
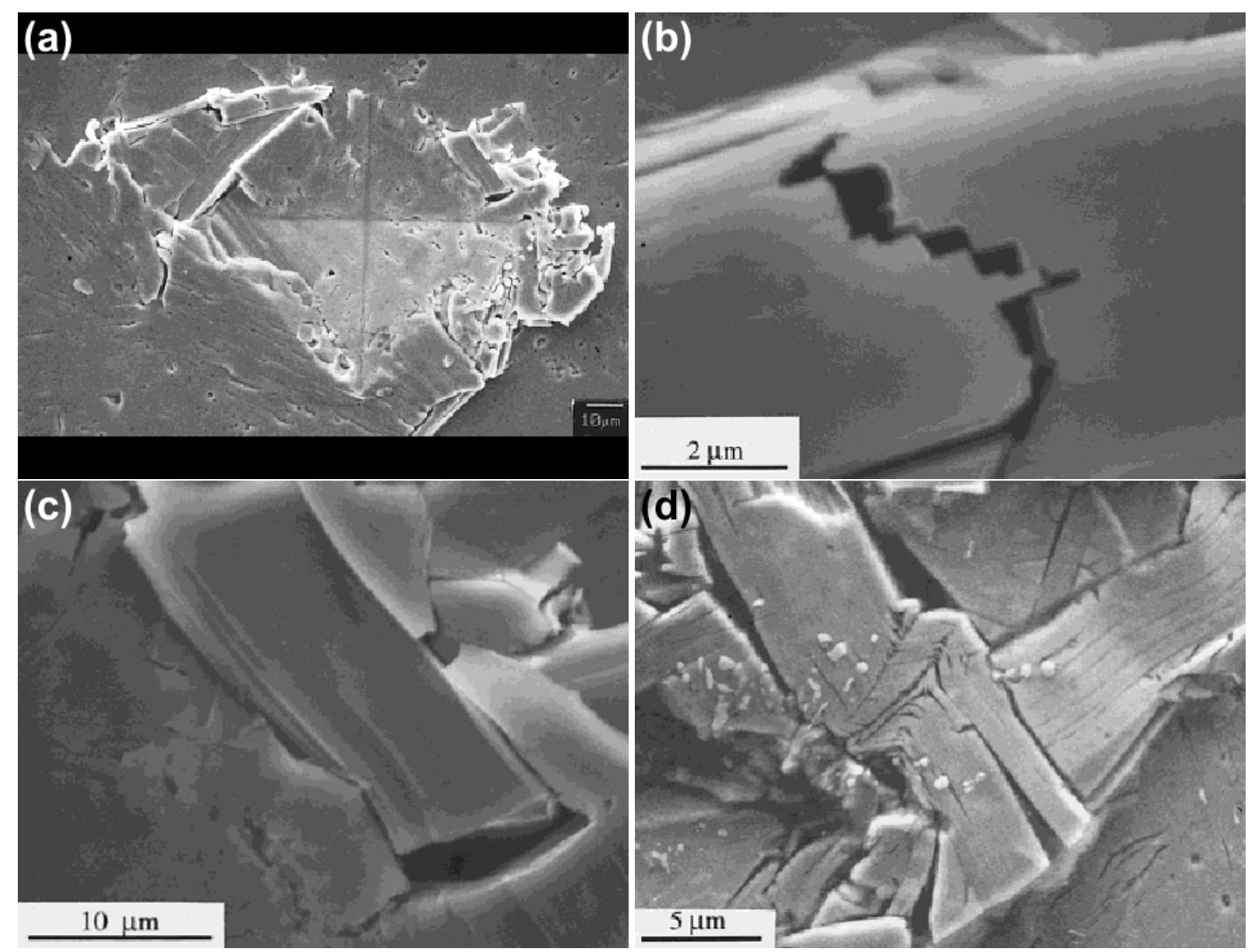

Abbildung 2.4: Das Verhalten der MAX-Nanolaminate unter mechanischer Belastung. Im Gegensatz zu normalen Keramiken ist die strukturelle Schädigung eng auf das Gebiet der Belastung (hier Eindruck eines Vickers-Härteprüfers $F=10 \mathrm{~N}$ ) begrenzt (a). Die Absorption der mechanischen Energie erfolgt durch Brüche entlang der Schichten in der Kristallstruktur (b), Ausreißen einzelner Körner (c) und Stauchung von Körnern mit Schichtspaltung (d) [26].

Eine weitere vorteilhafte Eigenschaft der MAX-Phasen ist ihre hohe thermische und chemische Stabilität. Erst bei Temperaturen oberhalb von $1000{ }^{\circ} \mathrm{C}$ findet eine merkliche Oxidation statt. Die geschwindigkeitsbestimmenden Schritte sind dabei die Diffusion von Sauerstoff in das und von den M- und A-Elementen aus dem Material. Dabei entsteht an der Oberfläche eine passivierende Schicht aus vor allem M- und teilweise A-Oxiden, die eine weitere Oxidation hemmen [27, 28]. MAX-Phasen schmelzen nicht, sondern zerfallen bei entsprechenden Temperaturen peritektisch nach der Gleichung:

$$
\mathrm{M}_{\mathrm{n}+1} \mathrm{AX}_{\mathrm{n}} \rightarrow \mathrm{M}_{\mathrm{n}+1} \mathrm{X}_{\mathrm{n}}+\mathrm{A}
$$


Die Temperaturen, bei denen dieser Zerfall stattfindet, sind abhängig von der chemischen Zusammensetzung der MAX-Verbindungen und reichen von ca. $1000 \mathrm{~K}$ ( $\mathrm{Cr}_{2} \mathrm{GaN}$ ) [29] bis über $2600 \mathrm{~K}\left(\mathrm{Ti}_{3} \mathrm{SiC}_{2}\right)$ [30]. Außerdem führen Verunreinigungen in der chemischen Zusammensetzung - wie z.B. Eisen und Vanadium [31] oder Sauerstoff - [28] zu einer Absenkung der Zerfallstemperaturen.

Aus den hier beschriebenen Eigenschaften ergeben sich zahlreiche mögliche Anwendungen für MAX-Materialien:

- Strukturmaterialien für Hochtemperaturanwendungen $\left(\mathrm{Ti}_{3} \mathrm{SiC}_{2}\right.$ hat bei etwa halber Dichte die doppelte Steifigkeit von Nickelbasislegierungen und behält seine mechanischen Eigenschaften über einen höheren Temperaturbereich.)

- Ersatz für zerspanbare Keramiken (Die meisten Keramiken benötigen einen Sinterschritt nach der mechanischen Bearbeitung, der zu einer Volumenreduktion führt. MAX-Keramiken können im fertigen Zustand bearbeitet werden.)

- Verschleißschutzbeschichtungen $\left(\mathrm{Ti}_{3} \mathrm{SiC}_{2}\right.$ lässt sich nachträglich silizieren bzw. carburisieren [32]. Dies führt zu Erhöhungen der Oberflächenhärte auf 12 GPa bzw. 25 GPa.)

- Elektrische Kontakte in aggressiven Umgebungen (Die relativ hohe elektrische Leitfähigkeit der MAX-Phasen gepaart mit ihrer guten Oxidationsbeständigkeit ermöglicht einen Einsatz als Kontaktschichten in Umgebungen mit z.B. heißem Wasserdampf.)

- Oberflächen mit niedrigem Reibungskoeffizient (Gelingt es, Oberflächen aus hochorientiertem kristallinen MAX-Material herzustellen, kann man den ungewöhlich niedrigen Reibungskoeffizienten entlang der Basisflächen in reibungskritischen Anwendungen ausnutzen.)

Die Festkörpersynthese von MAX-Phasen erfolgt in der Regel aus den Elementen bzw. ihren Carbiden oder Nitriden bei Temperaturen über $1000^{\circ} \mathrm{C}$ und verhältnismäßig hohen Drücken, um kompakte und einphasige Produkte zu erhalten. Als eine Standardsynthesemethode für MAX-Vollmaterial hat sich mittlerweile Heißpressen, bzw. heißes isostatisches Pressen etabliert [10, 20, 33, 34]. Weitere mögliche Synthesewege sind Plasmasintern [35] und Vakuumsintern [36].

Dünne Schichten aus MAX-Phasen wurden ebenfalls bereits synthetisiert. Die dabei verwendete Methode war in allen Fällen das Magnetronsputtern [37-44]. Problematisch ist dabei lediglich, dass sich die MAX-Phasen nur bei sehr hohen Depositionstemperaturen von etwa $700-900{ }^{\circ} \mathrm{C}$ bilden. Im System Cr-Al-C gelang allerdings die Abscheidung 
von Schichten mit $90 \% \mathrm{Cr}_{2} \mathrm{AlC}-\mathrm{MAX}$-Anteil bei $450{ }^{\circ} \mathrm{C}$ [42]. Mittlerweile ist es auch gelungen, $\mathrm{Ti}_{2} \mathrm{AlC}-\mathrm{MAX}$-Schichten mittels Hochgeschwindigkeits-Flammspritzen herzustellen [45]. Zur Synthese dünner MAX-Schichten mit Hilfe der Pulslaserdeposition gibt es bis jetzt nur sehr wenige Veröffentlichungen. Bei der PLD von einem $\mathrm{Ti}_{3} \mathrm{SiC}_{2}$-Target erhielten Lange et al. lediglich Schichten, die aus einer Mischung von kristallinem TiC und Silizium bestanden [46]. Acquaviva et al. zeigten die Entstehung der Phase $\operatorname{Ti}_{3} \mathrm{Al}_{2} \mathrm{~N}_{2}$ bei der reaktiven PLD eines TiAl-Targets in einer Stickstoffatmosphäre [47]. Später wurde dann gezeigt, dass $\mathrm{Ti}_{3} \mathrm{Al}_{2} \mathrm{~N}_{2}$ nicht existiert, sondern diese Phase statt dessen der MAX-Phase $\mathrm{Ti}_{4} \mathrm{AlN}_{3-x}$ entspricht [48-50]. Die erfolgreiche Herstellung von $\mathrm{Ti}_{3} \mathrm{SiC}_{2}$-Schichten durch PLD bei sehr moderaten Temperaturen von $100-300{ }^{\circ} \mathrm{C}$ wurde von $\mathrm{Hu}$ et al. in einer Veröffentlichung beschrieben [51]. Diese war jedoch in der Folge Gegenstand fachlicher Diskussionen $[52,53]$, die das behauptete Entstehen der MAX-Phase anzweifelten.

\subsubsection{Das System Ti - Si - C}

Abbildung 2.5 zeigt das ternäre Phasendiagramm im System Ti-Si-C bei $1250{ }^{\circ} \mathrm{C}$. In diesem System liegen zahlreiche stabile Phasen vor. Neben den reinen Elementen und ihren binären Verbindungen in verschiedenen stöchiometrischen Zusammensetzungen existieren im Gleichgewicht zwei ternäre Phasen: $\mathrm{Ti}_{5} \mathrm{Si}_{3} \mathrm{C}_{1-x}$ - eine feste Lösung von Kohlenstoff in $\mathrm{Ti}_{5} \mathrm{Si}_{3}$ - und die MAX-Phase $\mathrm{Ti}_{3} \mathrm{SiC}_{2}$.

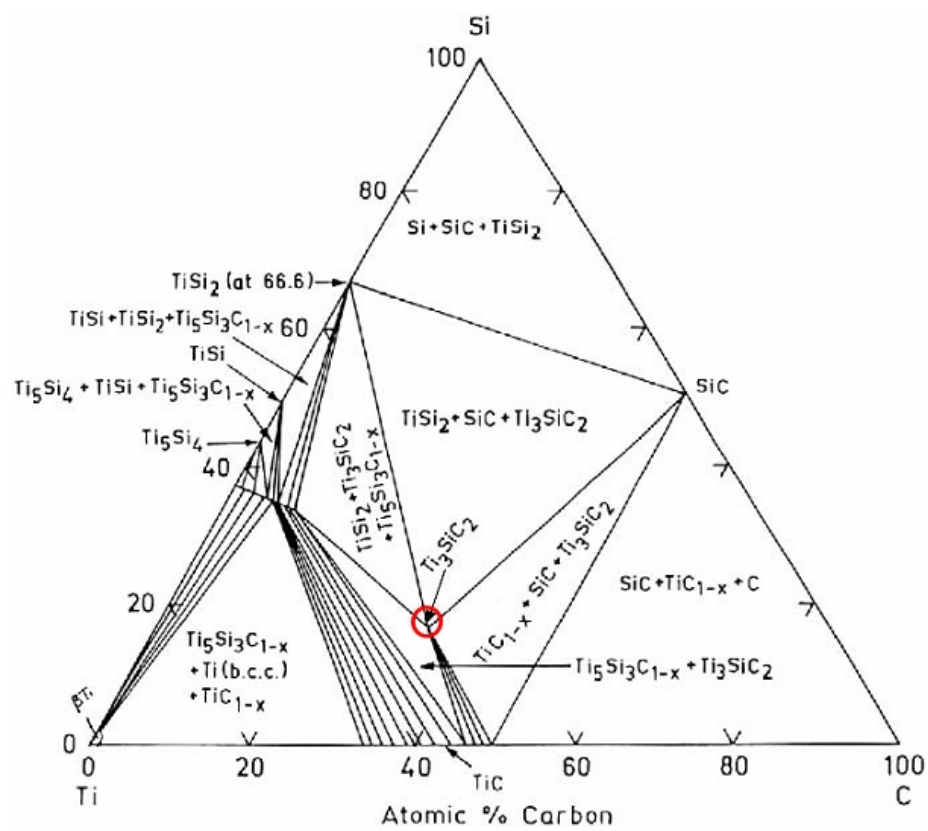

Abbildung 2.5: Der isothermale Schnitt durch das Ti-Si-C-Phasendiagram bei $1250{ }^{\circ} \mathrm{C}$. Die MAX-Phase $\mathrm{Ti}_{3} \mathrm{SiC}_{2}$ ist durch den roten Kreis gekennzeichnet [54].

Die MAX-Phase ist thermodynamisch stabil, verfügt aber über keinen Stabilitätsbereich. 
Außerhalb der stöchiometrischen Zusammensetzung von $\mathrm{Ti}_{3} \mathrm{SiC}_{2}$ (50 at.\% Ti, 17 at.- \% Si, 33 at.-\% C) tritt sie aber im Gemisch mit den entsprechenden anderen Randphasen auf.

$\mathrm{Ti}_{3} \mathrm{SiC}_{2}$ ist als prototypische Verbindung die bisher am besten untersuchte MAX-Phase. Die Gitterparameter der hexagonalen Elementarzelle sind $a=0,306 \mathrm{~nm}$ und $c=1,766 \mathrm{~nm}$. Die Dichte beträgt $\rho=4,54 \mathrm{~g} \cdot \mathrm{cm}^{-3}$. Die Wärmeleitfähigkeit bei Raumtemperatur liegt bei $k=33 \mathrm{~W} / \mathrm{m} \cdot \mathrm{K}$, die elektrische Leitfähigkeit bei $\sigma_{300 K}=4,5 \cdot 10^{6}(\Omega \cdot \mathrm{m})^{-1}$ und die Härte bei etwa $4 \mathrm{GPa}$. Der Elastizitätsmodul beträgt - abhängig von der Korngröße - E $\approx 335 \mathrm{GPa}$, der Schermodul $G \approx 140 \mathrm{GPa}[15]$.

Die Synthese von $\mathrm{Ti}_{3} \mathrm{SiC}_{2}$ als dünne Schicht wurde erfolgreich mit Hilfe des Magnetronsputterns durchgeführt. Palmquist et al. [37] und Emmerlich et al. [38] deponierten $\mathrm{Ti}_{3} \mathrm{SiC}_{2}$-Schichten auf $\mathrm{MgO}(111)$ und $\mathrm{Al}_{2} \mathrm{O}_{3}(0001)$ bei Temperaturen von 800-900 ${ }^{\circ} \mathrm{C}$. Die vorhergehende Deposition einer $\mathrm{TiC}_{x}$-Nukleationsschicht erwies sich dabei als für das Kristallwachstum hilfreich aber nicht zwingend notwendig.

Die in Abschnitt 2.1 beschriebene enge strukturelle Verwandschaft zwischen MAX-Phasen und ihren binären MX-Verbindungen lässt sich am Beispiel $\mathrm{Ti}_{3} \mathrm{SiC}_{2}$ und $\mathrm{TiC}$ sehr gut nachvollziehen. Auch die bereits oben erwähnte 1:1-Relation zwischen Gitterparameter $a$ der MAX-Phasen und dem M-M-Abstand in den binären MX-Verbindungen wird erfüllt. So ist $a\left(\mathrm{Ti}_{3} \mathrm{SiC}_{2}\right)=0,306 \mathrm{~nm}$ und in $\mathrm{TiC} d\left(\mathrm{Ti}_{E c k}-\mathrm{Ti}_{F Z}\right)=0,30597 \mathrm{~nm}$. $\mathrm{Ti}_{3} \mathrm{SiC}_{2}$ lässt sich also auch vereinfacht als kubisches $\mathrm{TiC}$ beschreiben, in das in regelmäßigen Abständen monoatomare Si-Schichten eingebaut sind (Abb. 2.6).

(a)

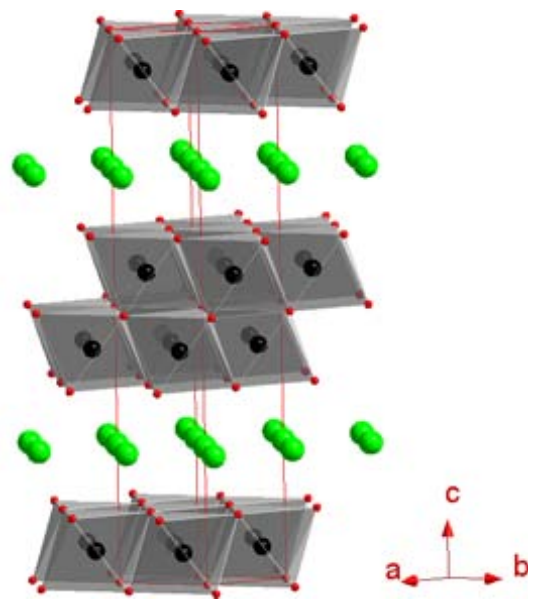

(b)

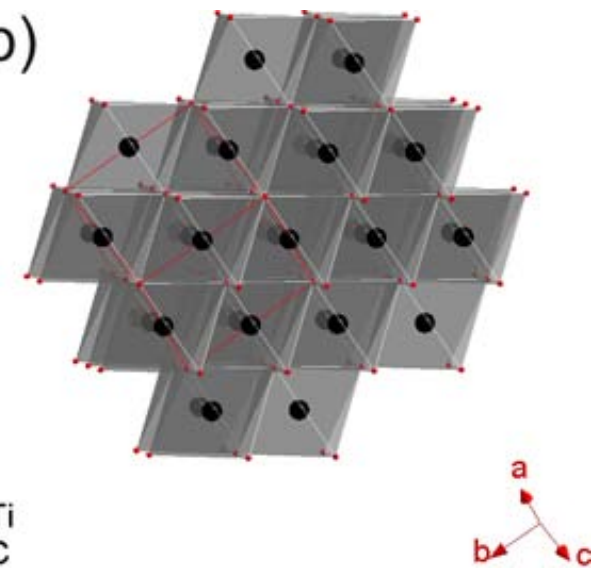

Abbildung 2.6: Vergleich der Kristallstrukturen von $\mathrm{Ti}_{3} \mathrm{SiC}_{2}$ (a) und $\mathrm{TiC}$ (b). Deutlich erkennbar sind die kantenverknüpften $\mathrm{Ti}_{6} \mathrm{C}$-Oktaeder als zentrale Strukturelemente in beiden Kristallen.

Tatsächlich lässt sich dieses Konzept von abwechselnd gestapelten $\mathrm{TiC}_{x^{-}}$und Si-Schichten noch weiter verfolgen. So beobachteten Palmquist et al. beim Deponieren von Ti-Si-CSchichten, dass sich Strukturen mit mehr als zwei $\mathrm{Ti}_{6} \mathrm{C}$-Schichten zwischen den Silizi- 
umschichten bildeten. Es entstanden also Schichtstrukturen mit Stapelfolgen, die in der Formulierung $\mathrm{M}_{n+1} \mathrm{AX}_{n}$ einem $n>2$ entsprechen $\left(\mathrm{Ti}_{3} \mathrm{SiC}_{2}: n=2\right)$ [55]. Außerdem wurden dünne Filme hergestellt, in denen die Anzahlen der $\mathrm{Ti}_{6} \mathrm{C}$-Schichten zwischen den Siliziumschichten unregelmäßig verteilt sind. Abbildung 2.7 zeigt die transmissionselektronenmikroskopische Aufnahme einer Ti-Si-C-Schicht mit unregelmäßiger Stapelfolge. Beobachtet wurden mindestens drei weitere Phasen, die entweder stabil oder metastabil sind. Diese sind: $\mathrm{Ti}_{4} \mathrm{SiC}_{3}\left(\mathrm{M}_{n+1} \mathrm{AX}_{n}\right.$ mit $\left.n=3\right)$, sowie $\mathrm{Ti}_{5} \mathrm{Si}_{2} \mathrm{C}_{3}$ und $\mathrm{Ti}_{7} \mathrm{Si}_{2} \mathrm{C}_{5}$. Die beiden letzteren haben nicht mehr die $\mathrm{M}_{n+1} \mathrm{AX}_{n}$-Zusammensetzung und in Folge dessen auch nicht mehr deren $P 6_{3} / m m c$-Symmetrie. Dennoch zeigen sie eine geordnete kristalline Struktur und lassen sich als Mischstrukturen von 211+312 ( $\left.\mathrm{Ti}_{5} \mathrm{Si}_{2} \mathrm{C}_{3}\right)$, bzw. 312+413 $\left(\mathrm{Ti}_{7} \mathrm{Si}_{2} \mathrm{C}_{5}\right)$ beschreiben. Theoretische Untersuchungen durch ab initio-Rechnungen [55] ergaben allerdings, dass diese beiden Phasen -zumindest bei $0 \mathrm{~K}$ - im Gleichgewicht nicht stabil sind. Die Stabilität dieser Phasen kann durch die freie Enthalpie $\Delta G$ der Zerfallsreaktionen

$$
\begin{gathered}
\mathrm{Ti}_{5} \mathrm{Si}_{2} \mathrm{C}_{3} \rightarrow \mathrm{x} \mathrm{Ti}_{3} \mathrm{SiC}_{2}+\mathrm{y} \mathrm{TiSi} i_{2}+\mathrm{z} \mathrm{Ti}_{5} \mathrm{Si}_{3} \text { bzw. } \\
\mathrm{Ti}_{7} \mathrm{Si}_{2} \mathrm{C}_{5} \rightarrow 2 \mathrm{Ti}_{3} \mathrm{SiC}_{2}+\mathrm{TiC}
\end{gathered}
$$

beschrieben werden. Ist $\Delta G<0$, sind die Verbindungen nicht stabil. Für Betrachtungen bei $T>0$ benötigt man aber nach

$$
\Delta \mathrm{G}=\Delta \mathrm{H}-\mathrm{T} \cdot \Delta \mathrm{S}
$$

Angaben zur Temperaturabhängigkeit der Reaktionsenthalpie $\Delta H$ und zu Entropieeffekten. Beides wurde in die Rechnungen nicht mit eingeschlossen. Insofern lassen sich noch keine endgültigen Aussagen darüber treffen, ob die beobachteten Phasen bei höheren Temperaturen stabil sind, oder ob ihr Zerfall in den dünnen Schichten lediglich kinetisch gehindert ist. Die erfolgreiche Synthese der 211-Phase $\mathrm{Ti}_{2} \mathrm{SiC}$ wurde bisher noch nicht beschrieben.

Auch die thermische Stabilität von dünnen $\mathrm{Ti}_{3} \mathrm{SiC}_{2}$-Schichten war bereits Gegenstand wissenschaftlicher Untersuchungen. Dabei stellten Emmerlich et al. fest, dass sich die Schichten bei Temperaturen von $1100-1200{ }^{\circ} \mathrm{C}$ entsprechend der Gleichung

$$
\mathrm{Ti}_{3} \mathrm{SiC}_{2} \rightarrow \mathrm{Ti}_{0,67} \mathrm{C}+\mathrm{Si}
$$

nach dem in Abb. 2.3 gezeigten Mechanismus zersetzen [56]. Das Silizium wird dabei als flüchtiges Siliziummonoxid $\mathrm{SiO}$ aus der Schicht entfernt. Im zurückbleibenden $\mathrm{Ti}_{0,67} \mathrm{C}$ werden durch die Ausbildung von Leerstellen im Gefüge die Kristallstruktur entspannt 


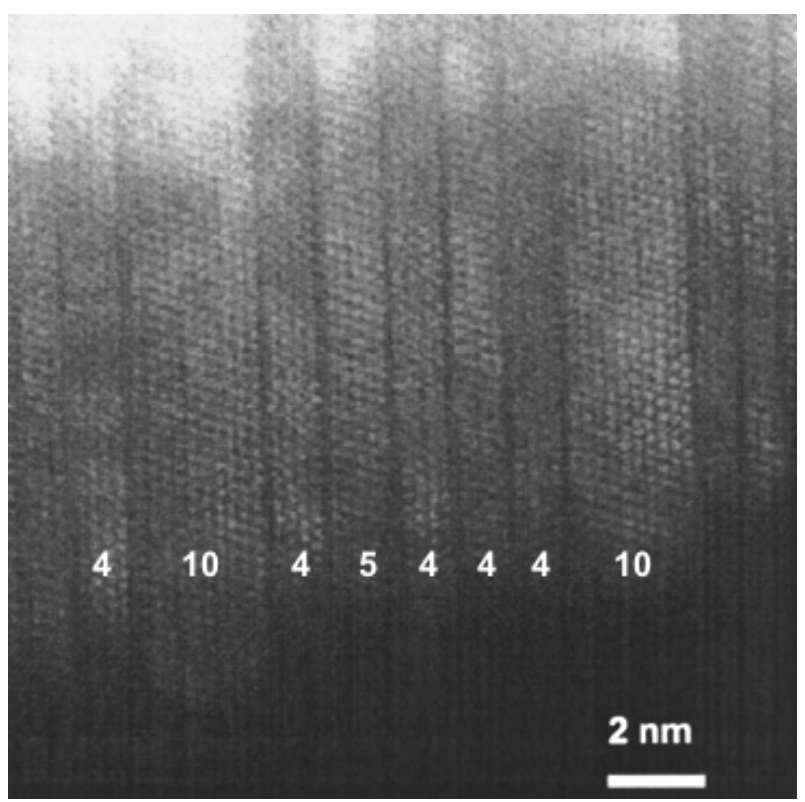

Abbildung 2.7: Transmissionselektronenmikroskopische Aufnahme einer Ti-Si-C-Schicht mit unregelmäßiger Stapelfolge. Die Zahlen geben die Anzahl der jeweiligen Ti-Atomschichten an [55].

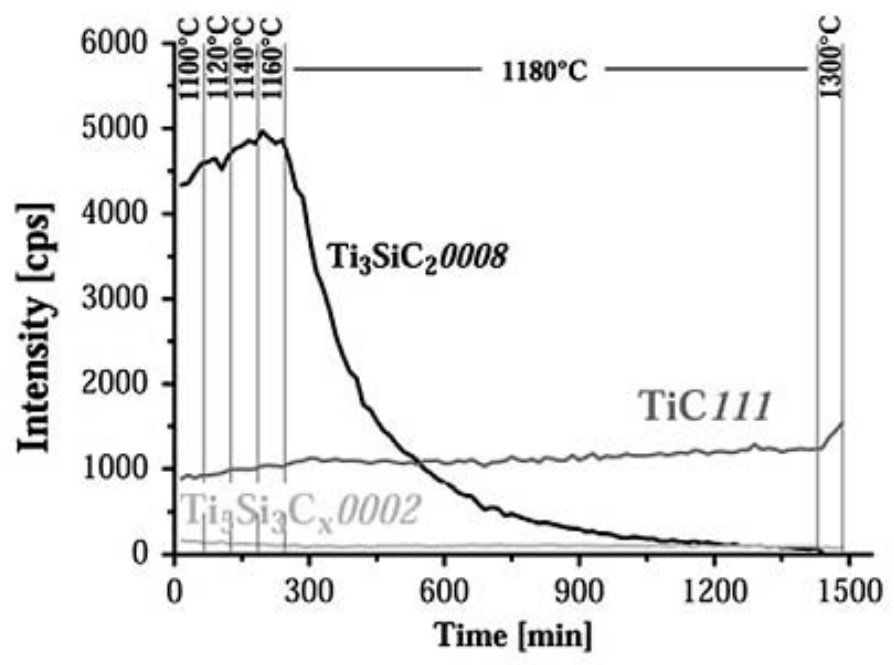

Abbildung 2.8: Die Entwicklung der Reflexintensitäten von $\mathrm{Ti}_{3} \mathrm{SiC}_{2} 0008$ und $\mathrm{TiC} 111$ einer $\mathrm{Ti}_{3} \mathrm{SiC}_{2}$-Schicht in Abhängigkeit der Heizrate von $1100-1300{ }^{\circ} \mathrm{C}$ [56].

und die Verzwillingungen abgebaut. Bei Temperaturen oberhalb von $1300{ }^{\circ} \mathrm{C}$ rekristallisiert es dann in der TiC-Struktur. Anhand der Intensitäten der Röntgenbeugungsreflexe $\mathrm{Ti}_{3} \mathrm{SiC}_{2} 0008$ und $\mathrm{TiC} 111$ (s. Abb. 2.8) konnten diese Vorgänge nachvollzogen und durch hochauflösende TEM-Aufnahmen bestätigt werden.

Beim Magnetronsputtern von einem $\mathrm{Ti}_{3} \mathrm{SiC}_{2}$-Target stellten Eklund et al. fest, dass die hergestellten Schichten bezüglich der $\mathrm{Ti}_{3} \mathrm{SiC}_{2}$-Zusammensetzung einen Kohlenstoffüberschuss von bis zu 50 at.- $\% \mathrm{C}$ hatten, der die Bildung von $\mathrm{Ti}_{3} \mathrm{SiC}_{2}$-MAX-Schichten ver- 
hindert. Um diesen C-Überschuss zu kompensieren wurden deswegen zwei verschiedene Strategien angewandt. Zum einen wurde gleichzeitig $\mathrm{zu} \mathrm{Ti}_{3} \mathrm{SiC}_{2}$ noch reines $\mathrm{Ti}$ abgeschieden. Dieses diente als Kohlenstoffsenke, und in den Schichten bildeten sich anschließend eine Mischung aus den MAX-Phasen $\mathrm{Ti}_{3} \mathrm{SiC}_{2}$ und $\mathrm{Ti}_{4} \mathrm{SiC}_{3}$, sowie $\mathrm{TiC}_{x}(x<1)$, das den überschüssigen Kohlenstoff bindet. Da auf diese Weise aber keine phasenreinen MAXSchichten hergestellt werden konnten, wurde das Konzept einer C-Senke noch verfeinert. Und zwar durch Deposition einer kohlenstoffarmen $\mathrm{TiC}_{x}$-Pufferschicht vor der Abscheidung der MAX-Phase. Tatsächlich konnte nun gezeigt werden, dass der die Bildung von $\mathrm{Ti}_{3} \mathrm{SiC}_{2}$ verhindernde Kohlenstoff in die titanreiche $\mathrm{TiC}_{x}$-Schicht diffundiert und auf diese Weise eine phasenreine $\mathrm{Ti}_{3} \mathrm{SiC}_{2}$-Schicht wachsen kann. Wenn die Dicke dieser $\mathrm{Ti}_{3} \mathrm{SiC}_{2^{-}}$ Schicht allerdings die Diffusionslänge des Kohlenstoffs überschreitet, endet das Wachstum der MAX-Phase. Statt dessen bildet sich auf der $\mathrm{Ti}_{3} \mathrm{SiC}_{2}$-Schicht wiederum TiC. In Abbildung 2.9 ist eine raster-transmissionselektronenmikroskopische (STEM) Aufnahme dieses $\mathrm{TiC}_{x^{-}}-\mathrm{Ti}_{3} \mathrm{SiC}_{2}$-TiC-Schichtpaketes dargestellt.

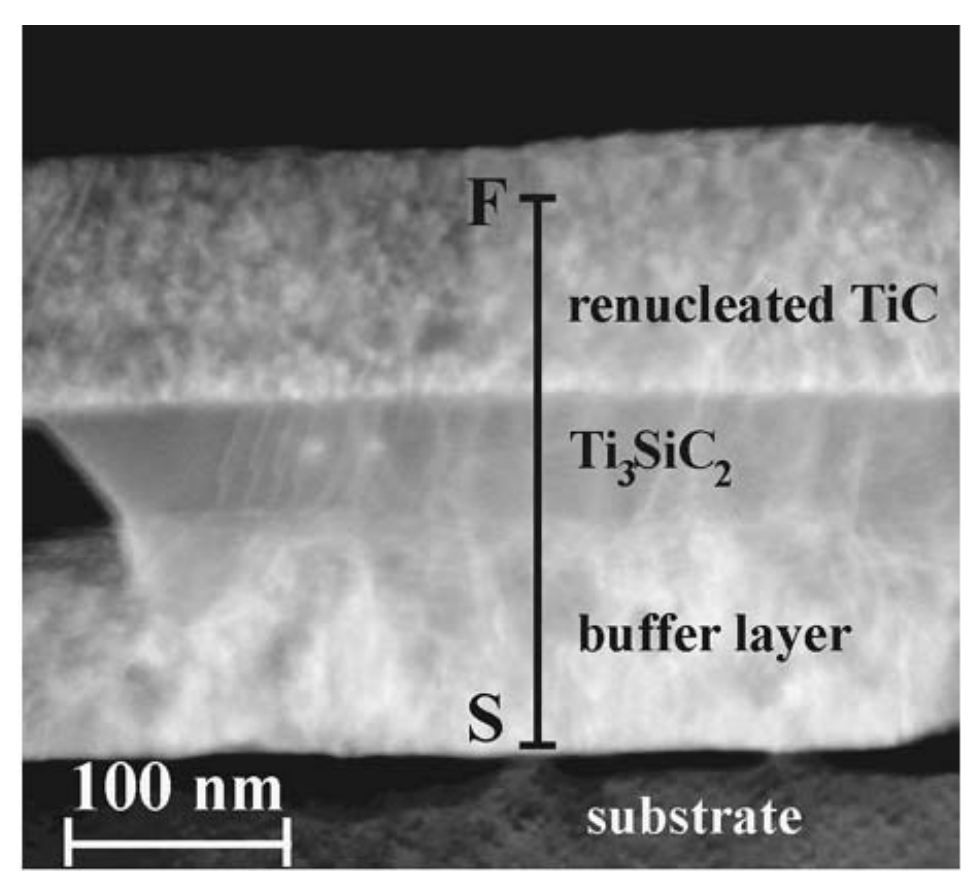

Abbildung 2.9: STEM-Aufnahme einer dünnen $\mathrm{Ti}_{3} \mathrm{SiC}_{2}$-Schicht, die auf einer $\mathrm{TiC}_{x}$-Pufferschicht deponiert wurde. Ab einer bestimmten Dicke des MAX-Phasen-Films kann der überschüssige Kohlenstoff nicht mehr in die Pufferschicht diffundieren, und es bildet sich an der Oberfläche wiederum $\mathrm{TiC}$ [44].

Bezüglich der Anwendung als Funktionsschichten ist interessant, dass im System Ti-Si-C bereits nanokristalline Schichten hergestellt wurden, in denen zwar keine MAX-Phasen gebildet wurden, die aber dennoch hervorragende elektrische und mechanische Eigenschaften aufwiesen. So synthetisierten Eklund et al. durch Magnetronsputtern aus einem $\mathrm{Ti}_{3} \mathrm{SiC}_{2^{-}}$ Target Komposit-Schichten aus nanokristallinem ( $\left.n c^{-}\right)$TiC und amorphem (a-) SiC [57]. 
Diese Schichten waren zum einen mit $H=20 \mathrm{GPa}$ außergewöhnlich hart. (So genannte superharte Nanokompositmaterialien sind bereits seit langem von hohem wissenschaftlichem und wirtschaftlichem Interesse [58].) Zum anderen zeigten sie erstaunlich gute elektrische Eigenschaften. Ein Vergleich des Kontaktwiderstandes - die letztendlich entscheidende Größe bei elektrischen Kontaktverbindungen - zeigte, dass der Widerstand zwischen $n c$ $\mathrm{TiC} / a$-SiC und Silber besonders bei Kontaktkräften oberhalb 100 N nur wenig größer ist als der zwischen Silber und Silber (Abb. 2.10). Gleichzeitig ist die Schicht aber wesentlich härter und somit verschleißbeständiger als eine Silberschicht. Diese Eigenschaften machen $n c$-TiC/a-SiC-Schichten interessant für eine kommerzielle Anwendung, welche von der schwedischen Firma ImpactCoatings (http: \\en.impactcoatings.se) bereits betrieben wird.

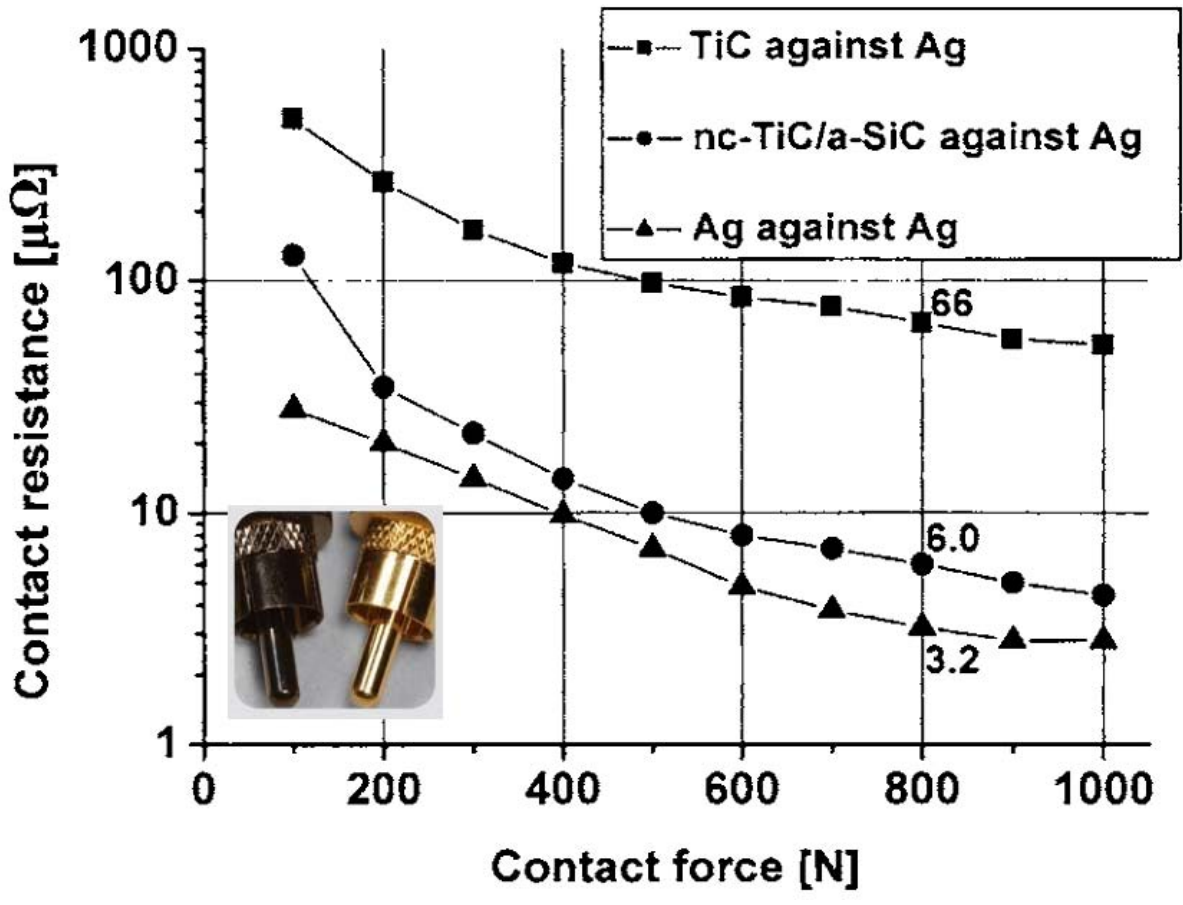

Abbildung 2.10: Vergleich der Kontaktwiderstände zwischen $\mathrm{TiC}, n c-\mathrm{TiC} / a-\mathrm{SiC}$ und Silber gegen Silber als Funktion der Kontaktkraft. Die Zahlenwerte geben den Kontaktwiderstand in $[\mu \Omega]$ bei $F=800 \mathrm{~N}$ an [57]. Unten links eingeklinkt: Audio-Cinchstecker mit Gold(rechts) und (links) Ti/Si/C-Beschichtung (Quelle: http: \\en.impactcoatings.se).

\subsubsection{Das System Cr - Al - C}

Das ternäre Phasendiagramm im System Cr-Al-C bei $1000{ }^{\circ} \mathrm{C}$ ist in Abbildung 2.11 dargestellt. Der obere Teil für Kohlenstoffanteile größer 50 at.-\% ist weggelassen, da in diesem Bereich keine weiteren stabilen Phasen zu finden sind. Chrom, Aluminium und Kohlenstoff entmischen sich in diesem Bereich immer $\mathrm{zu} \mathrm{Al}_{4} \mathrm{C}_{3}, \mathrm{Cr}_{3} \mathrm{C}_{2}$ und $\mathrm{C}$. Thermodynamisch 
stabile Phasen im System sind neben den reinen Elementen die drei Chromcarbide $\mathrm{Cr}_{3} \mathrm{C}_{2}$, $\mathrm{Cr}_{7} \mathrm{C}_{3}$ und $\mathrm{Cr}_{23} \mathrm{C}_{6}$, das einzige stabile Aluminiumcarbid $\mathrm{Al}_{4} \mathrm{C}_{3}$, die drei intermetallischen Al-Cr-Verbindungen $\mathrm{CrAl}_{4}, \mathrm{Cr}_{4} \mathrm{Al}_{9}$ und $\mathrm{Cr}_{5} \mathrm{Al}_{8}$, sowie die MAX-Phase $\mathrm{Cr}_{2} \mathrm{AlC}$. Zu beachten ist dabei, dass unterhalb von $1000{ }^{\circ} \mathrm{C}$ noch weitere $\mathrm{Al}-\mathrm{Cr}$-Legierungen $\left(\mathrm{AlCr}_{2}, \mathrm{Al}_{7} \mathrm{Cr}\right.$, $\mathrm{Al}_{11} \mathrm{Cr}_{2}$ ) existieren, die jedoch bei $1000{ }^{\circ} \mathrm{C}$ nicht mehr stabil sind [59]. Außerdem existiert eine metastabile Phase $\mathrm{CrC}$, die in einer kubisch-flächenzentrierten NaCl-Struktur $(F m \overline{3} m)$ mit dem Gitterparameter $a=0,410 \mathrm{~nm}$ kristallisiert [60]. Diese Phase ist insofern interessant, als dass sie bezüglich der MAX-Phase $\mathrm{Cr}_{2} \mathrm{AlC}$ das Analogon zu den binären kubischen MX-Phasen darstellt. Theoretische Berechnungen zur elektronischen Dichteverteilung (Abb. 2.12) zeigen die starke Ähnlichkeit zwischen den Cr-C-Bindungen in $\mathrm{Cr}_{2} \mathrm{AlC}$ und $\mathrm{CrC}$ [61]. Grundsätzlich ließe sich also auch zwischen $\mathrm{Cr}_{2} \mathrm{AlC}$ und $\mathrm{CrC}$ eine Strukturbeziehung wie die in Abbildung 2.6 zeigen. Auch das Strukturkriterium der 1:1-Relation zwischen Gitterparamater $a$ von $\mathrm{Cr}_{2} \mathrm{AlC}$ und dem M-M-Abstand in $\mathrm{CrC}$ wird erfüllt. So ist $a\left(\mathrm{Cr}_{2} \mathrm{AlC}\right)=0,285 \mathrm{~nm}$ und in $\mathrm{CrC}$ ist $d\left(\mathrm{Cr}_{E c k}-\mathrm{Cr}_{F Z}\right)=0,290 \mathrm{~nm}$.

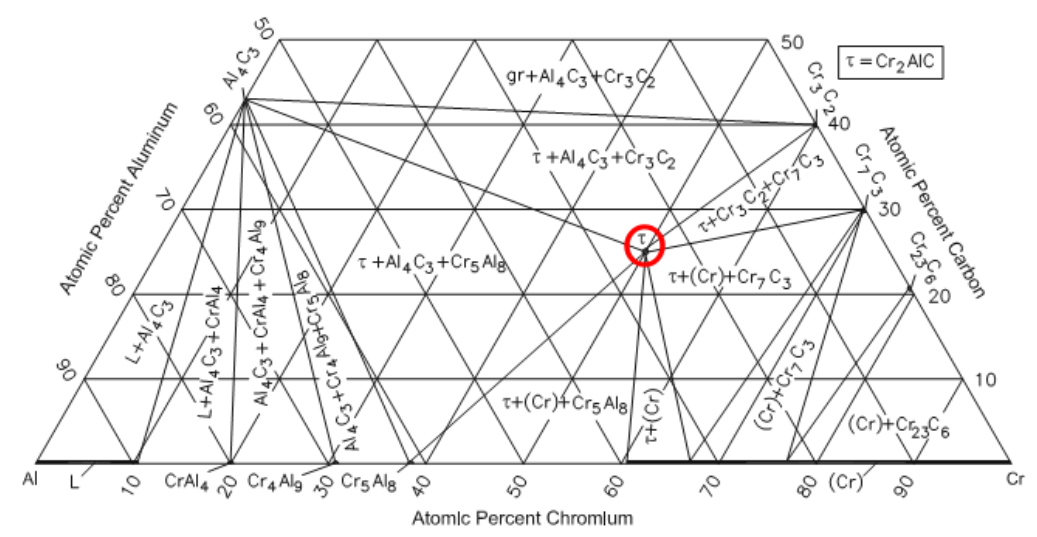

Abbildung 2.11: Der isothermale Schnitt durch das Cr-Al-C-Phasendiagram bei $1000{ }^{\circ} \mathrm{C}$. Die MAX-Phase $\mathrm{Cr}_{2} \mathrm{AlC}$-hier $\tau$ genannt- ist durch den roten Kreis gekennzeichnet [62] nach [63].

Allerdings ist $\mathrm{CrC}$ eben nicht thermodynamisch stabil. An einen Zerfall von $\mathrm{Cr}_{2} \mathrm{AlC}$ nach

$$
\mathrm{M}_{\mathrm{n}+1} \mathrm{AX}_{\mathrm{n}} \rightarrow \mathrm{M}_{\mathrm{n}+1} \mathrm{X}_{\mathrm{n}}+\mathrm{A}
$$

muss sich also eine weitere Umwandlung

$$
\mathrm{Cr}_{2} \mathrm{C} \rightarrow \mathrm{x} \mathrm{Cr}_{3} \mathrm{C}_{2}+\mathrm{y} \mathrm{Cr}_{7} \mathrm{C}_{3}
$$

anschließen.

Die Gitterparameter der $\mathrm{Cr}_{2}$ AlC-Elementarzelle sind $a=0,285 \mathrm{~nm}$ und $c=1,282 \mathrm{~nm}[6]$ bei einer Dichte von $\rho=5,25 \mathrm{~g} \cdot \mathrm{cm}^{-3}$. In Abhängigkeit der Mikrostruktur beträgt die 

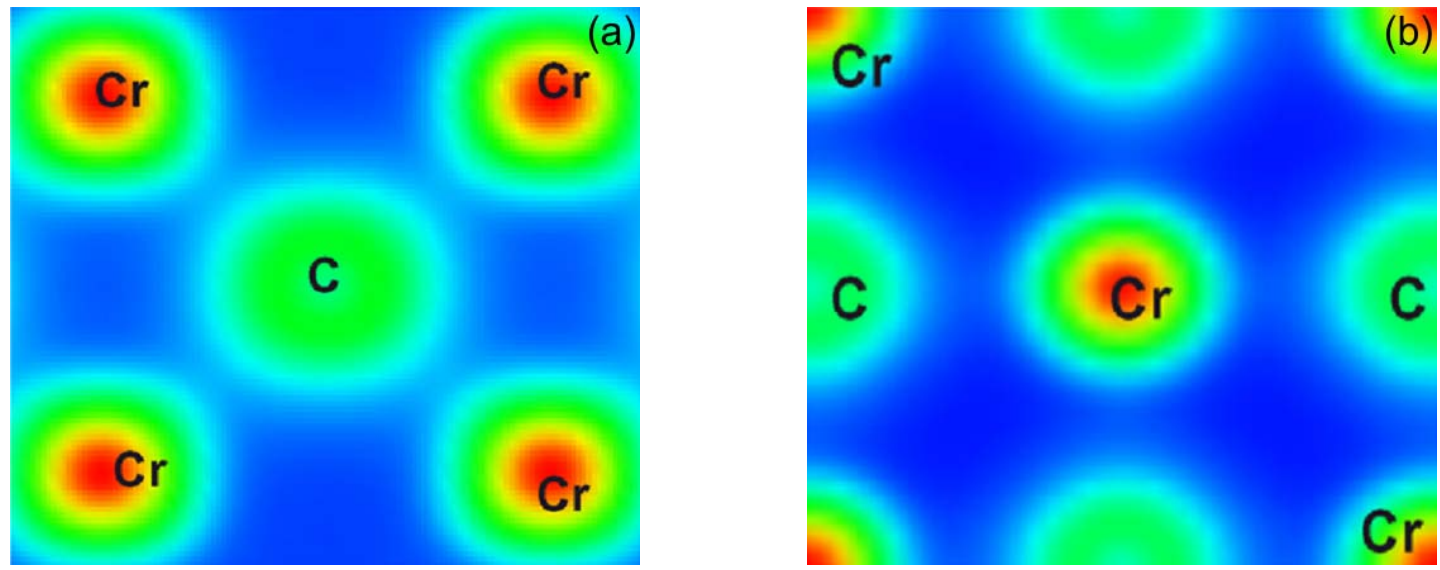

Abbildung 2.12: Vergleich der Elektronendichteverteilungen in $\mathrm{Cr}_{2} \mathrm{AlC}$ (a) und in der metastabilen CrC-Struktur (b). Die Elektronendichte variiert von 0,10 (dunkelblau) bis 3,62 (dunkelrot) Elektronen $/ \AA^{3}[61]$.

Härte von $\mathrm{Cr}_{2} \mathrm{AlC}$ 3,5-5,5 GPa. Die elektrische und die Wärmeleitfähigkeit liegen bei $k=17,5 \mathrm{~W} / \mathrm{m} \cdot \mathrm{K}$ beziehungsweise $\sigma=1,4 \cdot 10^{6}(\Omega \cdot \mathrm{m})^{-1}$ Damit ist $\mathrm{Cr}_{2} \mathrm{AlC}$ etwas härter als $\mathrm{Ti}_{3} \mathrm{SiC}_{2}$, leitet aber Strom und Wärme weniger gut. Der Elastizitätsmodul beträgt - als eine Funktion der Korngröße $-E \approx 280 \mathrm{GPa}$, der Schermodul $G \approx 120 \mathrm{GPa}$ [64].

Die Synthese von dünnen $\mathrm{Cr}_{2}$ AlC-Schichten durch Magnetronsputtern wurde bereits beschrieben. Dabei wurde einerseits aus Aluminium-, Chrom- und Kohlenstoff-Elementtargets auf $\mathrm{Si}(111)$-Substrate [65] und andererseits aus einem $\mathrm{Cr}_{2} \mathrm{AlC}$-Target auf Edelstahl gesputtert [42]. Im ersten Fall handelte es sich um eine so genannte kombinatorische Synthese. Das heißt, dass in der Schicht auf dem Substrat mehrere Bereiche mit unterschiedlicher stöchiometrischer Zusammensetzung vorliegen. Dabei fanden Mertens et al. heraus, dass entgegen den Vorhersagen aus dem Phasendiagramm ein Homogenitätsbereich in Richtung eines erhöhten Al-Anteils für die $\mathrm{Cr}_{2} \mathrm{AlC}-$ Phase existiert. Allerdings wurden diese Untersuchungen lediglich anhand von Röntgenbeugungsmessungen durchgeführt. Insofern ist es auch möglich, dass eine entspechende Kompensationsphase sich dem Nachweis - durch Segregation in Korngrenzen oder als amorphe Phase - entzieht. Im zweiten Fall, dem Sputtern eines $\mathrm{Cr}_{2}$ AlC-Targets auf Edelstahl, gelang Walter et al. die Synthese kristallinen $\mathrm{Cr}_{2} \mathrm{AlC}-$ Filmen bei relativ moderaten Temperaturen von $450{ }^{\circ} \mathrm{C}$. Die Phasenreinheit der Schichten betrug dabei über $90 \% \mathrm{Cr}_{2} \mathrm{AlC}$. Bemerkenswert bei diesen Experimenten ist die relativ ungewöhnliche Geometrie. Die Substratoberfläche war um $40^{\circ}$ gegenüber dem Magnetron geneigt, was darauf hindeutet, dass auch die Inhomogenität des Magnetronplasmas einen Einfluss auf die Phasenbildung während der Abscheidung hat. 


\subsubsection{Das System Ti - Al - N}

Auch im Dreistoffsystem Ti-Al-N existieren stabile Phasen mit der Zusammensetzung $\mathrm{Ti}_{n+1} \mathrm{AlN}_{n}$. Dabei handelt es sich um $\mathrm{Ti}_{2} \mathrm{AlN}_{1-x}$ und $\mathrm{Ti}_{4} \mathrm{AlN}_{3-x}$. Allerdings sind die $\mathrm{Zu}-$ sammenhänge hier etwas komplizierter als in den oben beschriebenen Systemen Ti-Si-C und Cr-Al-C. In Abbildung 2.13 sind isothermale Schnitte durch das Ti-Al-N-Phasendiagramm bei $1325{ }^{\circ} \mathrm{C}$ bzw. $900{ }^{\circ} \mathrm{C}$ dargestellt. Die beiden MAX-Phasen werden darin als $\tau_{2}=\mathrm{Ti}_{2} \mathrm{AlN}_{1-x}$ und $\tau_{3}=\mathrm{Ti}_{4} \mathrm{AlN}_{3-x}$ bezeichnet. Auffällig ist, dass beide Phasen unterstöchiometrische Zusammensetzungen in Bezug auf Stickstoff haben. So ist in $\mathrm{Ti}_{2} \mathrm{AlN}_{1-x} \quad x=0,18$, die Zusammensetzung von $\tau_{2}$ also $\mathrm{Ti}_{2} \mathrm{AlN}_{0,82}$ [66]. Erheblich diskutiert wurde außerdem die Struktur der Phase $\tau_{3}\left(\mathrm{Ti}_{4} \mathrm{AlN}_{3-x}\right)$. Zunächst beschrieben sie Lee und Petuskey als $\mathrm{Ti}_{3} \mathrm{Al}_{1-x} \mathrm{~N}_{2}$, eine aluminiumarmen Phase in der $\mathrm{Ti}_{3} \mathrm{SiC}_{2}$-Struktur [48]. Barsoum und Schuster stellten allerdings fest, dass der in [48] vorgeschlagene Gitterparameter $c=2,33350 \mathrm{~nm}$ mit der Kristallstruktur von $\mathrm{Ti}_{3} \mathrm{SiC}_{2}$ nicht kompatibel ist [67]. Nach erneuter Untersuchung mittels hochaufösender Transmissionelektronenmikroskopie, chemischer Analyse und Neutronenbeugungsexperimenten wurde die Struktur von $\tau_{3}$ von Barsoum et al. letztendlich als $\mathrm{Ti}_{4} \mathrm{AlN}_{3-x}$ mit $x=0,1$ - eine 413-MAX-Phase - angegeben $[49,50]$. Die Phase $\mathrm{Ti}_{4} \mathrm{AlN}_{3-x}$ ist nur in einem relativ kleinen Bereich zwischen $1250{ }^{\circ} \mathrm{C}$ und $1400{ }^{\circ} \mathrm{C}$ thermodynamisch stabil. Außerdem stellt sich das Gleichgewicht im System Ti-Al-N an der Zusammensetzung von $\mathrm{Ti}_{4} \mathrm{AlN}_{3-x}$ nur sehr langsam ein. Zur Synthese von $\mathrm{Ti}_{4} \mathrm{AlN}_{3-x}$ sind deshalb relativ lange Anlass-Zeiten notwendig. Bei diesen Anlass-Experimenten musste außerdem darauf geachtet werden, dass das Material sehr kompakt ist, weil sonst ein Verlust von Aluminium aus dem System beobachtet wurde $[48,68]$. Obwohl $\mathrm{Ti}_{4} \mathrm{AlN}_{3-x}$ nur bei hohen Temperaturen stabil ist, war es möglich die Phase nach der Synthese zu untersuchen. Offensichtlich ist der Zerfall von $\mathrm{Ti}_{4} \mathrm{AlN}_{3-x}$ also kinetisch stark gehemmt.

Tabelle 2.2 gibt einen Überblick über Gitterparameter und ausgewählte physikalische Eigenschaften der beiden MAX-Phasen $\mathrm{Ti}_{2} \mathrm{AlN}_{1-x}$ und $\mathrm{Ti}_{4} \mathrm{AlN}_{3-x}$. Der bei $\mathrm{Ti}_{3} \mathrm{SiC}_{2}$ und $\mathrm{Cr}_{2} \mathrm{AlC}$ erwähnte Zusammenhang zwischen dem Gitterparameter $a$ der MAX-Phase und dem Abstand zwischen dem M-Atom auf der Würfelecke und M-Atom in der Flächenmitte der kubischen MX-Verbindung existiert auch in den Phasen $\mathrm{Ti}_{2} \mathrm{AlN}_{1-x}$ und $\mathrm{Ti}_{4} \mathrm{AlN}_{3-x}$. In beiden MAX-Phasen ist $a=0,299 \mathrm{~nm}$, in TiN beträgt $d\left(\mathrm{Ti}_{E c k}-\mathrm{Ti}_{F Z}\right)=0,300 \mathrm{~nm}$. Bemerkenswert ist die mit $0,5 \cdot 10^{6}(\Omega \cdot \mathrm{m})^{-1}$ vergleichsweise sehr geringe elektrische Leitfähigkeit von $\mathrm{Ti}_{4} \mathrm{AlN}_{3-x}$. Diese ist auf die unterstöchiometrische Zusammensetzung der Phase zurückzuführen. Dabei ist allerdings nicht ein Mangel an Ladungsträgern der Grund für die schlechte elektrische Leitung. Statt dessen ist die Mobilität der Ladungsträger - vermutlich durch Streuung der Elektronen an den Stickstoff-Fehlstellen im Gitter - stark eingeschränkt [70]. Zumindest ist die effiziente Streuung von Elektronen an Gitterfehlstellen in Übergangsmetallcarbiden und -nitriden schon seit langem bekannt [71]. 


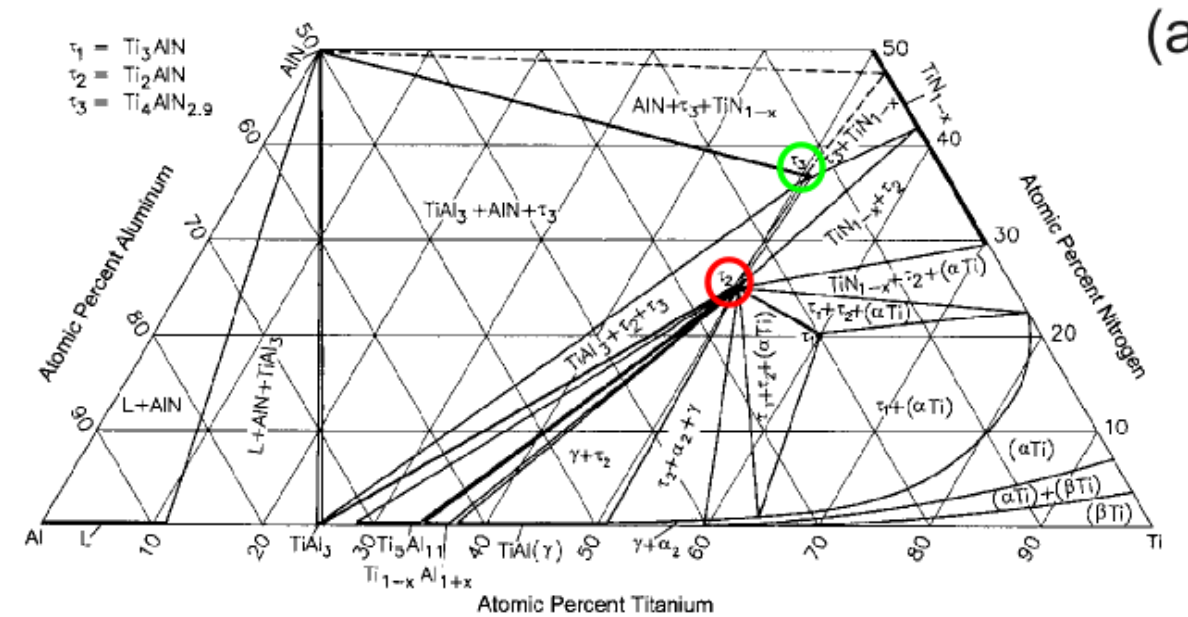

(a)

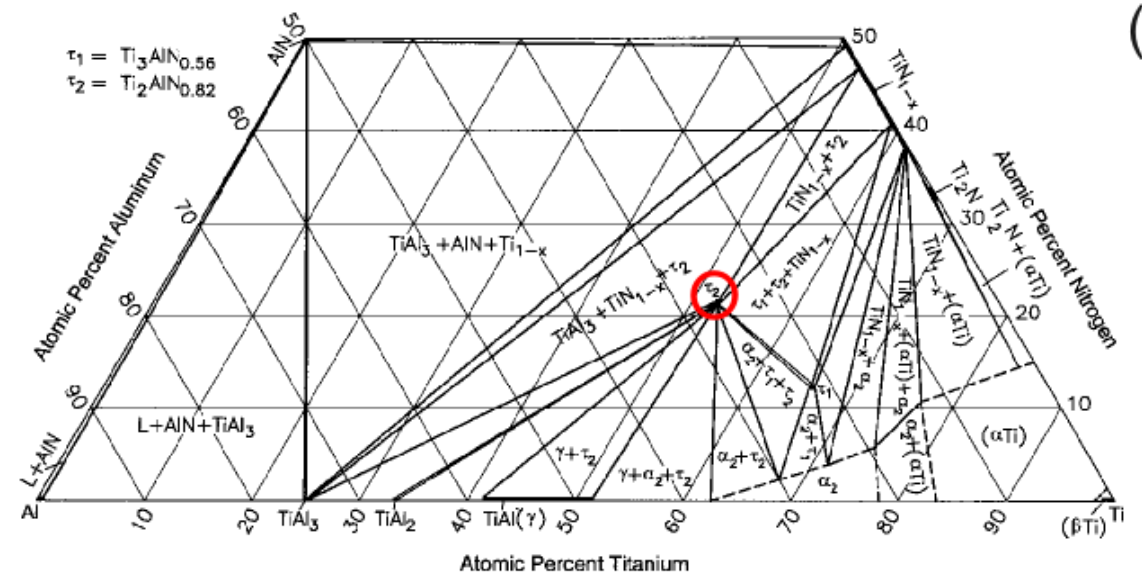

(b)

Abbildung 2.13: Isothermale Schnitte durch das Ti-Al-N-Phasendiagramm bei $1325^{\circ} \mathrm{C}$ (a) und $900{ }^{\circ} \mathrm{C}(\mathrm{b})$ mit den Phasen $\mathrm{Ti}_{2} \mathrm{AlN}_{1-x}=\tau_{2}$ (rote Kreise) und $\mathrm{Ti}_{4} \mathrm{AlN}_{3-x}=\tau_{3}$ (grüner Kreis). Die Phase $\tau_{3}$ ist bei $900{ }^{\circ} \mathrm{C}$ thermodynamisch nicht mehr stabil [69].

Tabelle 2.2: Gitterparameter und ausgewählte physikalische Eigenschaften von $\mathrm{Ti}_{2} \mathrm{AlN}_{1-x}$ und $\mathrm{Ti}_{4} \mathrm{AlN}_{3-x}$ nach [4]. Die Werte für TiN und $\mathrm{Ti}_{3} \mathrm{SiC}_{2}$ sind zum Vergleich mit angegeben.

\begin{tabular}{|c|c|c|c|c|c|}
\hline Verbindung & $\begin{array}{c}\text { Gitter- } \\
\text { parameter } \\
{[\AA]}\end{array}$ & $\begin{array}{c}\text { Theoretische } \\
\text { Dichte } \\
{\left[\mathrm{g} \cdot \mathrm{cm}^{-3}\right]}\end{array}$ & $\begin{array}{c}\text { Elektrische } \\
\text { Leitfähigkeit } \\
{\left[(\Omega \cdot \mathrm{m})^{-1}\right]}\end{array}$ & $\begin{array}{l}\text { Härte } \\
{[\mathrm{GPa}]}\end{array}$ & $\begin{array}{c}\text { Elastizitäts- } \\
\text { modul } \\
{[\mathrm{GPa}]}\end{array}$ \\
\hline $\mathrm{Ti}_{2} \mathrm{AlN}_{1-x}$ & $\begin{array}{c}a=2,99 \\
c=13,61\end{array}$ & 4,31 & $3,2-4 \cdot 10^{6}$ & 3,5 & - \\
\hline $\mathrm{Ti}_{4} \mathrm{AlN}_{3-x}$ & $\begin{array}{c}a=2,99 \\
c=23,34\end{array}$ & 4,61 & $0,5 \cdot 10^{6}$ & 2,5 & $310 \pm 2$ \\
\hline $\mathrm{Ti}_{3} \mathrm{SiC}_{2}$ & $\begin{array}{c}a=3,07 \\
c=17,67\end{array}$ & 4,53 & $4,5 \cdot 10^{6}$ & 4 & 333 \\
\hline $\mathrm{TiN}$ & $a=4,24$ & 5,4 & $4 \cdot 10^{6}$ & $18-20$ & 251 \\
\hline
\end{tabular}


Erfolgreiche Synthesen dünner Schichten im System Ti-Al-N gelangen bisher nur mit der Phase $\mathrm{Ti}_{2} \mathrm{AlN}_{1-x}$. Joelsson et al. deponierten einkristalline $\mathrm{Ti}_{2} \mathrm{AlN}_{1-x}$-Schichten auf $\mathrm{MgO}(111)$ durch reaktives Magnetronsputtern eines 2Ti:Al-Targets in $\mathrm{Ar} / \mathrm{N}_{2}$-Atmosphäre bei einer Substrattemperatur von $830{ }^{\circ} \mathrm{C}$ [41]. Persson et al. synthetisierten epitaktische $\mathrm{Ti}_{2} \mathrm{AlN}_{1-x}(0001)$-Schichten durch reaktives $\mathrm{Ti} / \mathrm{Al}$-Doppeltarget-Magnetronsputtern in $\mathrm{Ar} / \mathrm{N}_{2}$-Atmosphäre auf $\mathrm{Al}_{2} \mathrm{O}_{3}(0001)$-Substraten bei $1050{ }^{\circ} \mathrm{C}$ [72]. Beckers et al. gelang die epitaktische Synthese von $\mathrm{Ti}_{2} \mathrm{AlN}_{1-x}$ Schichten auf einer $\left(\mathrm{Ti}_{0,63} \mathrm{Al}_{0,37}\right) \mathrm{N}-$ Nukleationsschicht auf $\mathrm{MgO}(111)$ - und $\mathrm{Al}_{2} \mathrm{O}_{3}(0001)$-Substraten durch reaktives Magnetronsputtern von Ti- und Al-Elementtargets in $\mathrm{Ar} / \mathrm{N}_{2}$-Atmosphäre bei $690{ }^{\circ} \mathrm{C}$ [43]. Bei anschließenden Untersuchungen zur thermischen Stabilität stellten sie fest, dass sich die $\mathrm{Ti}_{2} \mathrm{AlN}_{1-x^{-}}$ Schichten - im Gegensatz zur hohen thermischen Beständigkeit des $\mathrm{Ti}_{2} \mathrm{AlN}_{1-x^{-}}$Vollmaterials bereits bei Temperaturen von $800{ }^{\circ} \mathrm{C}$ zersetzen. Bei dieser Zersetzung wird den Schichten Aluminium durch Diffusion an die Oberfläche und anschließendes Verdampfen nach

$$
\mathrm{Ti}_{2} \mathrm{AlN}_{1-\mathrm{x}} \rightarrow \mathrm{Ti}_{2} \mathrm{~N}_{1-\mathrm{x}}+\mathrm{Al} \uparrow
$$

entzogen. Das verbleibende $\mathrm{Ti}_{2} \mathrm{~N}_{1-x}$ rekristallisiert anschließend wiederum zu unterstöchiometrisch zusammengesetztem kubischen $\operatorname{TiN}_{x}$. Ein bei $800{ }^{\circ} \mathrm{C}$ ebenfalls auftretender sehr breiter Reflex an der $\mathrm{Ti}_{4} \mathrm{AlN}_{3-x}$ (0004)-Position deutet darauf hin, dass im geringen Umfang ein nur partieller Verlust jeder zweiten Al-Monolage aus $\mathrm{Ti}_{2} \mathrm{AlN}_{1-x}$ zur Bildung einer stark stickstoffarmen $\mathrm{Ti}_{4} \mathrm{AlN}_{3-x}$-Phase mit $x=1$ nach

$$
2 \mathrm{Ti}_{2} \mathrm{AlN}_{1-\mathrm{x}} \rightarrow \mathrm{Ti}_{4} \mathrm{AlN}_{2}+\mathrm{Al} \uparrow
$$

führen kann [73]. Die Röntgendiffraktogramme dieser Untersuchungen sind in Abbildung 2.14 dargestellt. Dabei ist anzumerken, dass die Zerfallstemperatur der $\mathrm{Ti}_{2} \mathrm{AlN}_{1-x^{-}}$ Schichten aus [73] auf dem gleichen Substrat mit $800{ }^{\circ} \mathrm{C}$ deutlich unterhalb der $\mathrm{Ti}_{2} \mathrm{AlN}_{1-x^{-}}$ Synthesetemperatur von $1050{ }^{\circ} \mathrm{C}$ aus [72] liegt. Eine Erklärung für dieses Phänomen steht bisher noch aus.

Für die Nukleation und das Wachstum von $\mathrm{Ti}_{2} \mathrm{AlN}_{1-x}$ auf $\mathrm{MgO}(100)$-Substraten beim reaktiven Magnetronsputtern wurde von Beckers et al. ein Wachstumsmodell vorgeschlagen. Dieses ist in Abbildung 2.15 dargerstellt und besteht im Wesentlichen aus vier Schritten [74]:

- Titan, Aluminum und Stickstoff aus dem Magnetronplasma bilden auf der MgOOberfläche zunächst die metastabile kubische Mischphase $\left(\mathrm{Ti}_{1-x} \mathrm{Al}_{x}\right) \mathrm{N}_{y}$. Diese Phase entmischt sich im Folgenden spinodal zu kubischem $\mathrm{TiN}_{y^{\prime}}$ und wurtzitischem $\mathrm{AlN}_{y^{\prime \prime}}$. Spinodale Entmischungen von $(\mathrm{Ti}, \mathrm{Al}) \mathrm{N}-\mathrm{Mischphasen}$ wurden bereits in früheren Experimenten beobachtet und beschrieben [75]. 


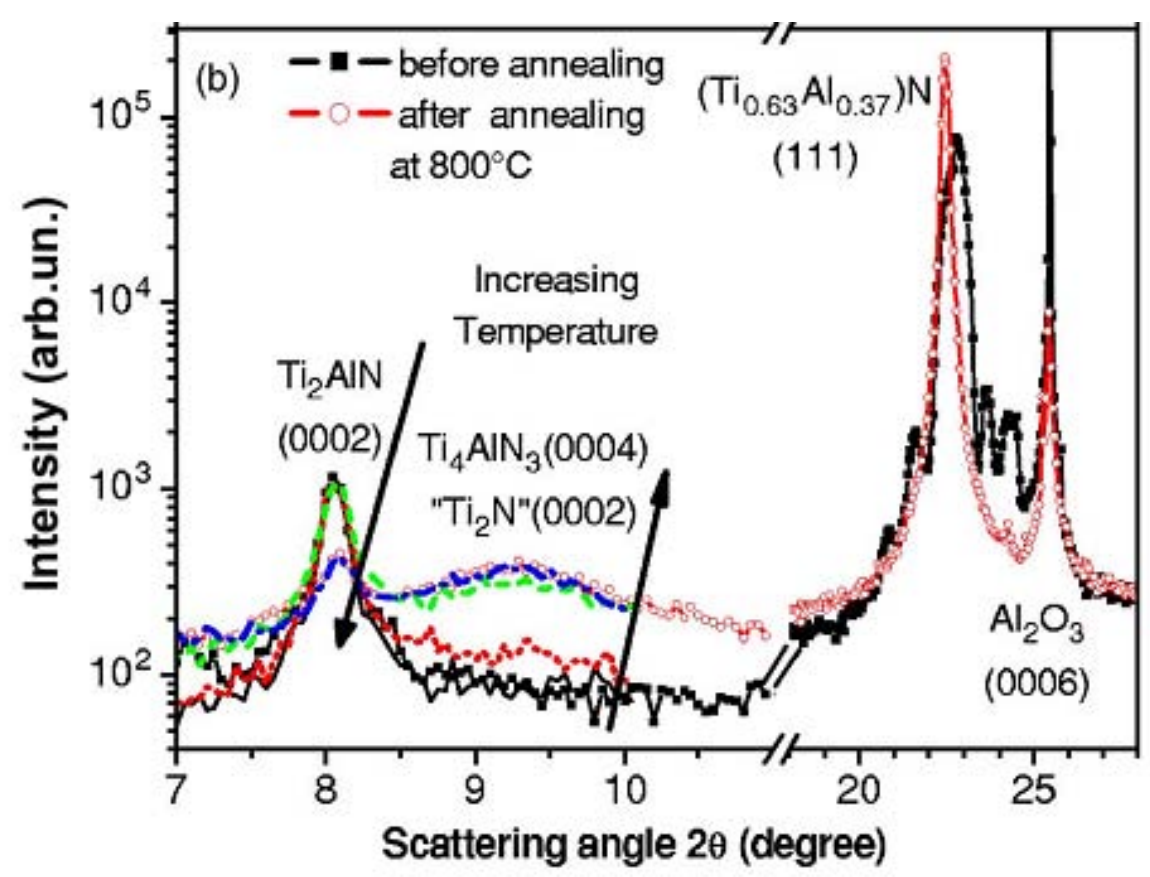

Abbildung 2.14: In situ Röntgendiffraktogramme einer $50 \mathrm{~nm}$ dicken $\mathrm{Ti}_{2} \mathrm{AlN}_{1-x}$-Schicht auf $\mathrm{Al}_{2} \mathrm{O}_{3}(0001)$ nach der Deposition bei $675{ }^{\circ} \mathrm{C}$ (schwarze Quadrate) und nach $1 \mathrm{~h}$ Anlassen bei $800^{\circ} \mathrm{C}$ (rote Kreise). Zusätzlich sind beim zwischenzeitlich gemessene Beugungsaufnahmen bei $700{ }^{\circ} \mathrm{C}$ (rote Punkte), $750{ }^{\circ} \mathrm{C}$ (grüne Striche) und $800{ }^{\circ} \mathrm{C}$ (blaue Strich-Punkte) angegeben. Grafik nach [73].

- An der Grenzfläche zum Substrat diffundieren $\mathrm{Ti}$ und $\mathrm{Al}$ in das, $\mathrm{Mg}$ dagegen aus dem Substrat und bilden dabei ein $\mathrm{Mg}_{2}(\mathrm{Al}: \mathrm{Ti}) \mathrm{O}_{4}$-Spinell. Durch die Diffusion von Stickstoff nach innen kommt es auf den $\mathrm{AlN}_{y^{\prime \prime}}$-Domänen zur Keimbildung von $\mathrm{Ti}_{2} \mathrm{AlN}_{1-x}$. Dabei dienen Al-Atomschichten des $\mathrm{AlN}_{y^{\prime \prime}}$ als Grundlage für eine $\mathrm{Ti}_{2} \mathrm{AlN}_{1-x}$-Elementarzelle.

- Durch kontinuierliches Wachstum des Spinells und der $\mathrm{Ti}_{2} \mathrm{AlN}_{1-x}$-Phase wird die Dicke der anfänglichen $\left(\mathrm{Ti}_{1-x} \mathrm{Al}_{x}\right) \mathrm{N}_{y}$-Schicht weiter verringert. Beim Wachstum der $\mathrm{Ti}_{2} \mathrm{AlN}_{1-x}$-Schicht dient das darunter liegende $\left(\mathrm{Ti}_{1-x} \mathrm{Al}_{x}\right) \mathrm{N}_{y}$ als Senke für den überschüssigen Stickstoff aus dem Magnetronplasma. (Ein ähnlicher Zusammenhang zwischen Diffusion des X-Elements und Wachstum der MAX-Phase wurde bereits in Abschnitt 2.1.2 geschildert.)

- Das Spinell-Wachstum kommt durch Abbau der Konzentrationsgradienten zum Erliegen, während die polykristalline $\mathrm{Ti}_{2} \mathrm{AlN}_{1-x}$-Schicht unter weiterhin statt findenden Diffusionsprozessen an der $\mathrm{Ti}_{2} \mathrm{AlN}_{1-x}-\mathrm{TiN}_{y^{\prime}} / \mathrm{AlN}_{y^{\prime \prime}}$-Grenzschicht weiter wächst.

Eine Möglichkeit zur Herstellung von $\mathrm{Ti}_{2} \mathrm{AlN}_{1-x}$-Schichten bei Temperaturen von $500{ }^{\circ} \mathrm{C}$ beschrieben Höglund et al. Dabei wurden mit einem Magnetron zunächst Pakete von 
(a)

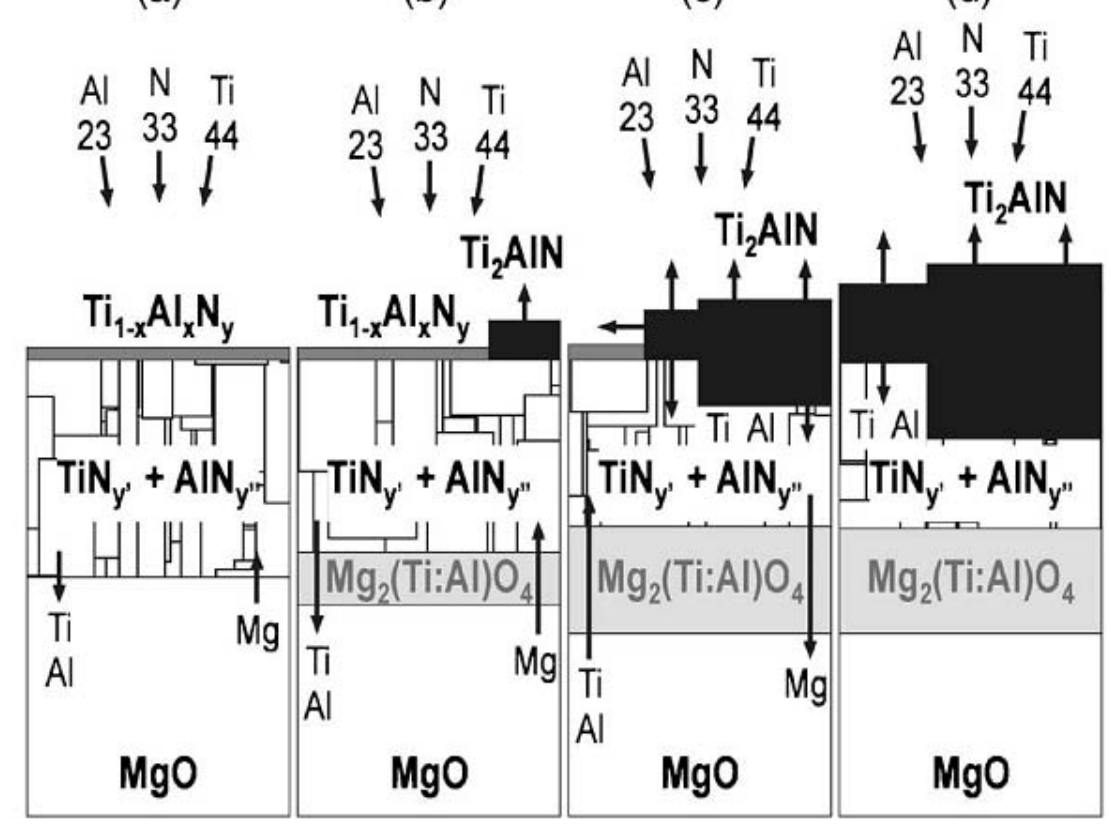

Abbildung 2.15: Modell des Schichtwachstums von $\mathrm{Ti}_{2} \mathrm{AlN}_{1-x}$ auf $\mathrm{MgO}(111)$. (a) Bildung und spinodaler Zerfall von $\left(\mathrm{Ti}_{1-x} \mathrm{Al}_{x}\right) \mathrm{N}_{y}$, (b) Formation des $\mathrm{Mg}_{2}(\mathrm{Al}: \mathrm{Ti}) \mathrm{O}_{4}$-Spinells und $\mathrm{Nu}$ kleation von $\mathrm{Ti}_{2} \mathrm{AlN}_{1-x}$, (c) weiteres Wachstum der $\mathrm{Ti}_{2} \mathrm{AlN}_{1-x}$-Schicht (durch Stickstoffdiffusion nach innen) und der Spinell-Grenzschicht, (d) Halt der Spinellbildung und weiteres Wachstum der polykristallinen $\mathrm{Ti}_{2} \mathrm{AlN}_{1-x^{-}}$Schicht durch Diffusionsprozesse an der $\mathrm{Ti}_{2} \mathrm{AlN}_{1-x^{-}}$ $\mathrm{TiN}_{y^{\prime}} / \mathrm{AlN}_{y^{\prime \prime}}$-Grenzschicht. Die Zahlen geben die Zusammensetzung der Gasphase in Atomprozent an [74].

dünnen Schichten aus abwechselnd Titan und wurtzitischem AlN bei $200{ }^{\circ} \mathrm{C}$ auf $\mathrm{Al}_{2} \mathrm{O}_{3}(0001)$ gesputtert. Aus diesen Schichtpaketen formiert sich dann in sehr kurzer Zeit bei $500{ }^{\circ} \mathrm{C}$ $\mathrm{Ti}_{2} \mathrm{AlN}_{1-x}$ durch eine Festkörperreaktion. Bei diesem Prozess spielen wiederum Diffusionprozesse - hier von $\mathrm{Al}$ und N - eine entscheidende Rolle. Vor allem die Dicke der zunächst deponierten Ti- und AlN-Schichten ist von besonderer Bedeutung [76].

Bei der reaktiven Pulslaserabscheidung (PLD) eines TiAl-Targets in Stickstoffatmosphäre beobachteten Acquaviva et al. neben der Formation von kubischem TiN auch die Entstehung einer Phase, die von ihnen als $\mathrm{Ti}_{3} \mathrm{Al}_{2} \mathrm{~N}_{2}$ identifiziert wurde. Spätere Untersuchungen ergaben dann allerdings, dass die Verbindung $\mathrm{Ti}_{3} \mathrm{Al}_{2} \mathrm{~N}_{2}$ nicht existiert. Statt dessen handelt es sich in diesem Fall um die MAX-Phase $\mathrm{Ti}_{4} \mathrm{AlN}_{3-x}[49,50]$. Als Grundlage für diese Identifizierung dienten allerdings nur Röntgendiffraktogramme, in denen die Reflexe für $\mathrm{Ti}_{3} \mathrm{Al}_{2} \mathrm{~N}_{2}$ indiziert wurden. Da sich die Raumgruppen von $\mathrm{Ti}_{3} \mathrm{Al}_{2} \mathrm{~N}_{2}\left(P 6_{3} m c\right)$ und $\mathrm{Ti}_{4} \mathrm{AlN}_{3-x}\left(P 6_{3} / m m c\right)$ aber unterscheiden, stimmen auch die Indizierungen nicht mehr überein. Insofern ist es nicht ganz klar, ob es sich hier mit Sicherheit um die MAX-Phase $\mathrm{Ti}_{4} \mathrm{AlN}_{3-x}$ handelte. 


\subsection{Pulslaserdeposition (Pulsed Laser Deposition)}

\subsubsection{Experimentelle Grundlagen}

Eine sehr ausführliche Darstellung zu den Grundlagen der Pulslaserdeposition findet sich in [77]. Hier sollen einige wichtige Teilaspekte daraus kurz dargestellt werden.

Im Jahr 1965 veröffentlichten H.M. Smith und A.F. Turner eine Publikation, in der sie die Möglichkeit beschrieben, verschiedene Materialen durch Laserverdampfung im Vakuum als dünne Schichten abzuscheiden [78]. Sie benutzten dazu einen gepulsten Rubinlaser mit einer Pulsenergie von $3 \mathrm{~J}$, einer Pulsdauer von etwa $1 \mathrm{~ms}$ und einer Wiederholrate von 1 Puls/min. Gegenüber bis dahin gebräuchlichen Vakuumverdampfungsmethoden hatte die Benutzung eines Lasers vor allem den Vorteil, ohne ein Heizen des zu verdampfenden Materials - und den damit verbundenen Nebenreaktionen wie thermische Zersetzung oder chemische Reaktionen mit dem Tiegelmaterial - auszukommen. Dadurch wurde es möglich, Vakuumabscheidungen wesentlich sauberer durchzuführen. Am Ende der Veröffentlichung kamen sie zu dem Schluss, dass die Vakuumverdampfung mit Hilfe eines Lasers durch Weiterentwicklung der Methode einige einzigartige Vorteile bieten kann. Dies war der Beginn der Entwicklung der Pulslaserdeposition (engl.: pulsed laser deposition, $P L D)$.

Grundsätzlich handelt es sich bei der PLD um eine konzeptionell und experimentell recht einfache Abscheidungsmethode. Eine Illustration des einfachsten PLD-Aufbaus ist in Abbildung 2.16 gezeigt. Der gepulste Laserstrahl wird fokussiert und auf ein Target in der Vakuumkammer gerichtet. Die sehr hohe Energiedichte des Laserstrahls bewirkt eine Verdampfung des Targetmaterials und die Bildung eines Ablationsplasmas. Die Bestandteile des Plasmas (Ionen, Atome, Moleküle, Atomcluster und größere feste Agglomerate) scheiden sich dann auf der Substratoberfläche als dünne Schicht ab. Der PLD-Prozess zeigt dabei Vor- und Nachteils gegenüber anderen Dünnschichttechniken wie Magnetronsputtern und Molekularstrahlepitaxie (MBE). Die Vorteile sind Flexibilität, schnelle Prozessführung und kongruente Verdampfung (siehe unten). Außerdem werden für die PLD keinerlei Heizelemente oder Entladungselektroden zur Verdampfung des Targetmaterials benötigt. Aus diesem Grund kann die PLD-Kammer mit beliebigen reaktiven Hintergrundgasen gefüllt werden; und dieses in einem größeren Druckbereich als bei anderen Beschichtungstungsverfahren (s. Abschnitt 2.2.3). Zu den Nachteilen gehören die Anwesenheit größerer $(\mu \mathrm{m})$ Agglomerate im Ablationsplasma und die relativ kleine räumliche Ausdehnung der Plasmafackel, die eine Beschichtung von größeren Substraten erschwert.

Obwohl die praktische Anwendung bereits 1965 gezeigt wurde, dauerte es bis in die 1980er Jahre, bis die Pulslaserabscheidung stärker ins Blickfeld der Forschung gelangte. Dies lag 


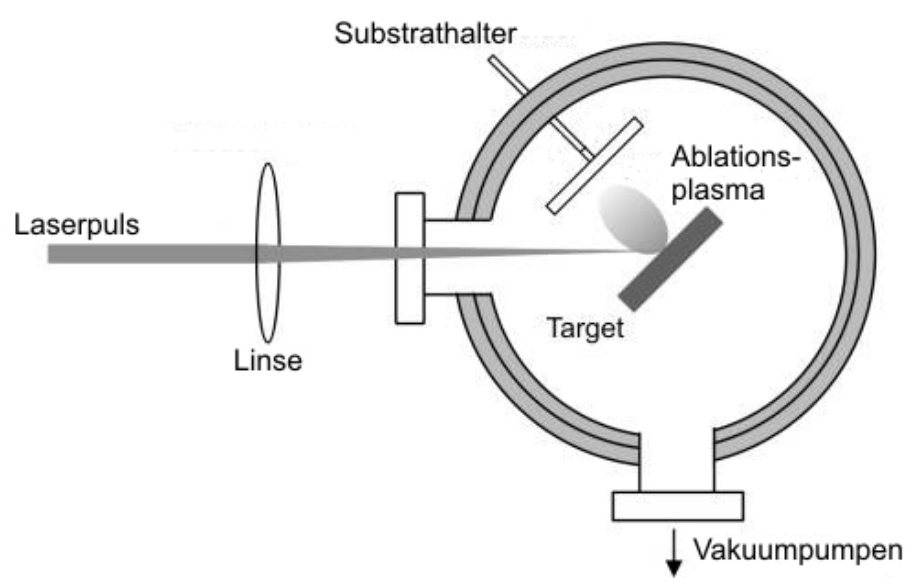

Abbildung 2.16: Schematische Zeichnung einer einfachen PLD-Anlage mit Strahlengang, Verdampfungstarget, Ablationsplasma und Substrat(halter).

zum einen daran, dass MBE als Konkurrenztechnik bereits stark etabliert war, und zum anderen daran, dass zu viele verschiedene Gruppen an zu vielen verschiedenen Materialien zur Laserverdampfung arbeiteten, ohne sich zusammenzuschließen und gemeinsam theoretische und praktische Untersuchungen zur PLD durchzuführen. Erst die erfolgreiche Synthese dünner Schichten aus dem Hochtemperatursupraleiter $\mathrm{YBa}_{2} \mathrm{Cu}_{3} \mathrm{O}_{7-x}[79,80]$ Ende der 1980er konnte von den Möglichkeiten der PLD überzeugen und damit eine erheblich gesteigerte Forschungsaktivität auslösen.

Die Attraktivität der PLD beruht im Wesentlichen auf zwei Eigenschaften: erstens, die Flexibilität bei der Anwendung. Seit den 1970er Jahren standen die ersten zuverlässigen elektronischen Güteschaltungen ( $Q$-switches) zur Verfügung, mit denen es möglich wurde, sehr kurze Laserpulse mit hohen Energiedichten bis zu $10^{8} \mathrm{~W} / \mathrm{cm}^{2}$ zu erzeugen. Betrachtet man die Formel

$$
E=\sqrt{\frac{2 \Phi}{c \epsilon_{0} n}}
$$

mit $E$ als dem elektrischen Feld des Laserlichtes in $[\mathrm{V} / \mathrm{cm}], \Phi$ als der Leistungsdichte in $\left[\mathrm{W} / \mathrm{cm}^{2}\right]$, der Dielektrizitätskonstante im Vakuum $\epsilon_{0}$, dem Brechungsindex $n$ des bestrahlten Materials und der Lichtgeschwindigkeit $c$, so erhält man für ein Material mit $n=1,5$ und einer Leistungsdichte $\Phi=5 \cdot 10^{8} \mathrm{~W} / \mathrm{cm}^{2}$ im bestrahlten Material eine elektrische Feldstärke von $5 \cdot 10^{5} \mathrm{~V} / \mathrm{cm}$. Bei der Absorption von Laserstrahlung dieser Stärke kommt es somit zur Bildung eines Plasmas im Target. Dadurch ist es möglich, mit Hilfe der PLD jede Substanz zu verdampfen, wenn die eingestrahlte - genauer: die absorbierte Laserleistung entsprechend hoch ist. Zweitens kommt es bei der PLD zur so genannten kongruenten Verdampfung. Das heißt, dass das Targetmaterial unter Erhaltung der chemischen Zusammensetzung in das Plasma überführt wird. Voraussetzung dafür ist, dass das 
erhitzte Volumen im Target - gegeben durch die thermische Diffusionslänge $L$ während des Laserpulses $L=2 \cdot \sqrt{D \cdot \tau}$ mit $D$ als der Wärmeleitzahl und $\tau$ der Laserpulslänge kleiner oder gleich der Dicke der ablatierten Schicht pro Puls ist. Erst diese kongruente Verdampfung ermöglicht es, so komplexe Verbindungen wie Y-Ba-Cu-O-Supraleiter als dünne Schichten aus dem entsprechenden Vollmaterial wie in [79] beschrieben zu synthetisieren. In diesem Zusammenhang ist es wichtig, ebenfalls zu erwähnen, dass kürzere Laser-Wellenlängen eine geringere Absorptionstiefe aufweisen. Auf diese Weise lassen sich durch die Verwendung von Frequenzvervielfachern das kongruente Verdampfen und die Aufrauung der Oberfläche durch die Ablation positiv beeinflussen.

Die Aufrauung der Targetoberfläche während der Laserablation stellt tatsächlich ein großes Problem dar, da aus dieser Aufrauung der größte Nachteil der PLD entsteht: das Auftreten größerer Partikel im $\mu$ m-Bereich im Ablationsplasma, in der Literatur als engl.: splashing bezeichnet. In den dünnen Schichten treten dadurch Inhomogenitäten durch kleine Partikel, die so genannten droplets, auf, die in Abbildung 2.17 gezeigt sind. Durch das splashing werden die Dünnschichten in ihrer Qualität erheblich vermindert. So kommt es bei Halbleiter-Filmen zur Bildung von Defekten und Streuzentren, die die Ladungsträgermobilität absenken und bei optischen Schichten zur Verringerung der Schadenstoleranz. Das Auftreten von splashing hat verschiedene Ursachen:

- Verdampfung unterhalb der Oberfläche (subsurface boiling)

- Flüssigkeitsausstoß durch den Plasma-Rückstoßdruck und

- Abblättern der Targetoberfläche

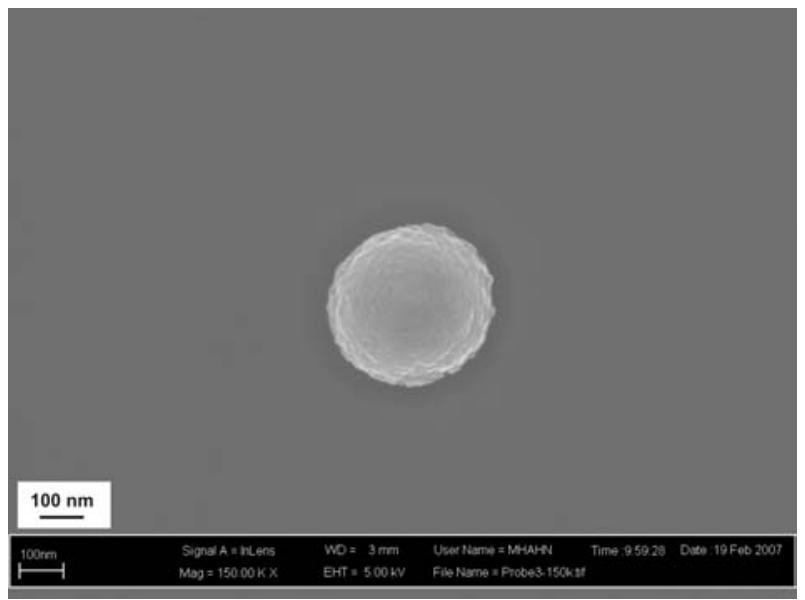

Abbildung 2.17: Droplet in einer TiC/Si-PLD-Schicht. Die Größe des Droplets ist hier unterhalb des $\mu$ m-Bereiches, da während der Deposition bereits Maßnahmen zur Verringerung der Dropletbildung getroffen wurden (Quelle: C. Lange, unveröffentlichte Ergebnisse). 
Die Verdampfung unterhalb der Oberfläche tritt auf, wenn die Zeit zur Umwandlung der Laserenergie in Wärme kürzer ist als die Zeit bis zur Verdampfung der Oberfläche. Dadurch wird das Material unterhalb der Oberfläche stark überhitzt, und es kommt zum Ausstoß kleiner Schmelzkugeln in die Gasphase, die sich dann in der dünnen Schicht niederschlagen. Dieser Effekt wird mit zunehmender Laserleistung stärker. Nach Schwarz und Tourtellotte [81] ist die maximale Laserleistungsdichte $\Phi_{\text {max }}$, die eine Materialoberfläche absorbieren kann, ohne dass es zum splashing kommt, in etwa

$$
\Phi_{\max }=\frac{\alpha \rho H_{e v}}{t_{r}}
$$

Dabei ist $\rho$ die Massendichte des Materials, $H_{e v}$ die Verdampfungswärme und $t_{r}$ die Relaxationszeit zum Transfer der eingestrahlten Energie ins Gitter. Der Faktor $\alpha$ ist eine Materialkonstante und beschreibt die Eindringtiefe des Lichtes in das Material. Er hängt von den elektrischen Eigenschaften des Targetmaterials zusammen und kann aus der elektrischen Leitfähigkeit $\sigma$, relativen Permeabilität $K_{m}$ und der Frequenz des Lichtes $f$ durch

$$
\alpha=\frac{252}{\sqrt{\sigma f K_{m}}}
$$

berechnet werden. Für dielektrische Materialien ist $\sigma$ klein und $\Phi_{\max }$ entprechend groß, so dass die Laserleistungsdichten der meisten PLD-Anwendungen für dielektrische Materialien unterhalb von $\Phi_{\max }$ liegen. In Metallen mit größerem $\sigma$ kann es dagegen bei Bestrahlung mit hoher Leistung zur Verdampfung unterhalb der Oberfläche kommen. Ein vergleichbarer Effekt, nämlich das Auswerfen von kleinen geschmolzenen Kugeln - jedoch aus anderen Gründen - tritt durch den Rückstoß bei der Ausbreitung des Plasmas auf. Keine geschmolzenen Kugeln, sondern zufällig geformte feste Partikel treten beim Abblättern des Targetmaterials auf. Durch die Ablation des Materials erodiert die Oberfläche des Targets zunehmend. Es bilden sich einige Mikrometer große nadelartige Strukturen, die zum Einfallswinkel des Laserlichtes hinzeigen [82]. Diese Dendriten sind sehr zerbrechlich und können daher durch den thermischen Schock der Laserstrahlung aus dem Target herausbrechen. Dann werden sie von der sich schnell ausdehnenden Plasmafackel mitgerissen und scheiden sich auf dem Substrat ab. Abbildung 2.18 zeigt eine elektronenmikroskopische Aufnahme dieser Strukturen in einem $\mathrm{ZrO}_{2}$-PLD-Target.

Eine genauere Untersuchung zur Entstehung und Vermeidung von droplets lieferten Fähler et al.. Sie stellten fest, dass Zahl und Größe der droplets von der Energiedichte des eingestrahlten Laserlichtes abhängen, und sich verschieden große Partikel räumlich unterschiedlich von der Targetoberfläche ausbreiten [83].

Zur Vermeidung des splashing wurden verschiedene Konzepte entwickelt. Eine Möglichkeit ist die Benutzung eines mechanischen Partikelfilters. Da die größeren Agglomerate in der 


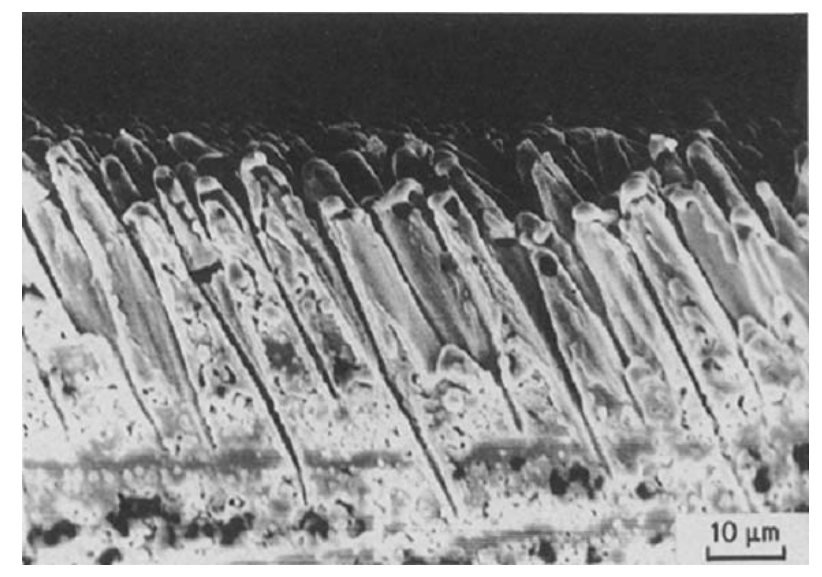

Abbildung 2.18: Querschliff eines $\mathrm{ZrO}_{2}$-PLD-Targets nach der Ablation. Die Dendriten sind zur Einfallsrichtung des Lasers hin geneigt. [82].

Plasmafackel viel langsamer sind, als einzelne Atome oder Atomcluster, kann man die unerwünschten Anteile über ihre Geschwindigkeit herausfiltern [84, 85]. Ein weiterer Ansatz ist die direkte Einflussnahme auf die Plasmafackel durch eine zweite synchronisierte PLD-Plasmafackel. Bei diesem Aufbau - der so genannten cross beam-PLD - werden zwei Plasmafackeln gekreuzt. Im Überschneidungsbereich der beiden Fackeln kommt es zu gasdynamischen Interaktionen, die eine Änderung der Ausbreitungsrichtung des Plasmas bewirken. Die langsamen und schweren $\mu$ m-Partikel durchqueren diesen Kreuzungsbereich weitestgehend ohne Änderung ihrer Flugbahn und werden auf diese Weise effizient aus dem Teilchenfluss zum Substrat entfernt [86]. Der Aufbau einer cross beam-PLD-Anlage ist in Abbildung 2.19 gezeigt. Eine ähnliche Wirkung erzielten Murakami et al., in dem sie die $\mu \mathrm{m}$-Bestandteile durch einen synchronisierten Gasstrahl aus der Plasmafackel bliesen $[87]$.

Der Nachteil dieser Techniken ist der hohe apparative Aufwand. Entweder ist es notwendig, einen motorisierten Aufbau zur mechanischen Teilchenfilterung in die PLD-Anlage einzubauen, oder man braucht einen zusätzlichen Laser, bzw. ein zusätzliches Gasventil, deren Betrieb dann auch noch sehr genau synchronisiert werden muss. Wesentlich einfacher - und ebenfalls recht effektiv in Hinblick auf die Teilchenentfernung aus dem Plasma - ist es, die Targetoberfläche so zu bewegen, dass der Laserstrahl eine großen Fläche abrastern kann. Die Oberfläche wird dabei während der Bestrahlung wesentlich weniger erodiert, und die Anzahl der Droplets auf dem Substrat folglich stark verringert. Diesen Effekt konnten Doughty et al. zeigen, in dem sie ein rotierendes PLD-Target einmal ohne und einmal mit einer zusätzlichen oszillierenden Bewegung bestrahlten [88]. Rasterkraftmikroskopische (AFM) Aufnahmen in 2.20 zeigen die deutliche Verringerung der Dropletzahl und -größe durch die oszillierende Bewegung des Targets während der Deposition. 


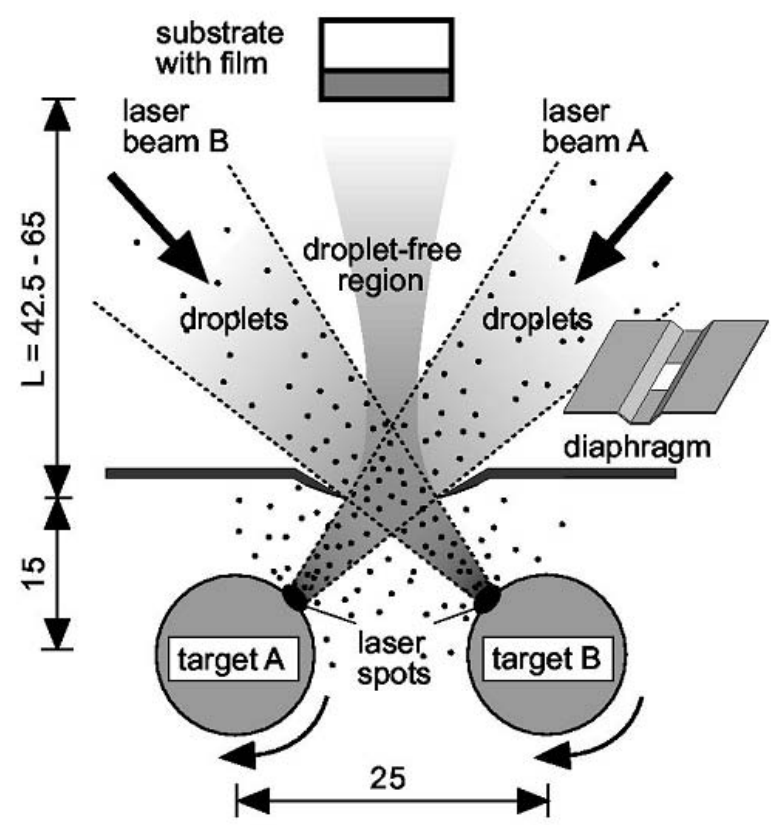

Abbildung 2.19: Aufbau einer cross beam-PLD-Anlage. Durch gasdynamische Interaktion der beiden Plasmafackeln kommt es zu einer Änderung der Ausbreitungsrichtung des Plasmas. Dadurch werden die großen und langsamen Agglomerate aus dem Depositionsfluss entfernt. Die Zahlenangaben sind Längen in [mm] [86].
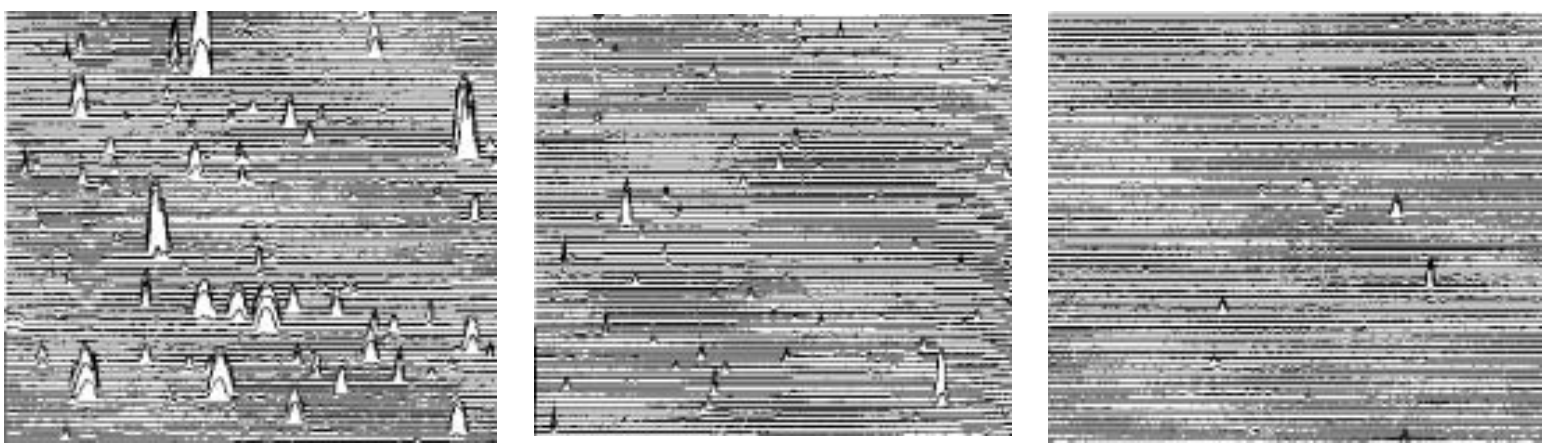

Abbildung 2.20: Vergleich der AFM-Aufnahmen $(50 \times 50 \mu \mathrm{m})$ dreier PLD-Schichten. Mitte: frisch polierte Targetoberfläche, links: nur kreisförmige Targetbewegung (einzelne Laserspur auf dem Target), rechts: oszillierende Targetbewegung (der Laserstrahl rastert eine große Targetoberfläche zufällig ab). Die Höhe der größten Erhebungen im linken Bild entspricht etwa $1 \mu \mathrm{m}[88]$.

\subsubsection{Eigenschaften des PLD-Plasmas}

Nach Fähler und Krebs [89] lässt sich die Verteilung der Geschwindigkeit der Ionen im Laserablationsplasma $f\left(v_{z}\right)$ als eine verschobene Maxwell-Boltzmann-Verteilung wie folgt beschreiben: 


$$
f\left(v_{z}\right) \propto v_{z}^{3} \cdot \exp \left(-\frac{m_{a} \cdot\left(v_{z}-u_{c m}\right)^{2}}{2 \cdot k_{b} \cdot T_{e f f}}\right)
$$

Dabei sind $u_{c m}$ die durchschnittliche Energie der Ionen, $m_{A}$ die Masse der Targetatome und $T_{\text {eff }}$ die effektive Temperatur. Bei der Ablation von Eisen erhielten sie - als lineare Funktion der eingestrahlten Laserfluenz - mittlere kinetische Energien von 70-150 eV bei Fluenzen von 5-10 J/ $\mathrm{cm}^{2}$. Dabei ist festzustellen, dass die kinetischen Energien auch von der Pulsdauer des Lasers abhängen. So verglichen Perrière et al. bei der PLD von Zinkoxid die Geschwindigkeiten in Femtosekunden $(f s)$ - mit denen in Nanosekunden $(n s)$ Ablationsplasmen [90]. Bei der $n s$-Ablation lagen die mittleren kinetischen Energien im Bereich von 5-15 eV, was sich mit den Größenordnungen aus [89] deckt. Bei der Ablation mit dem $f s$-Laser lagen die kinetischen Energien jedoch viel höher, nämlich 800-1000 eV, bei einer eingestrahlten Fluenz von etwa $9 \mathrm{~J} / \mathrm{cm}^{2}$. Offensichtlich spielt also auch die Zeitskala bei der Laser-Material-Wechselwirkung eine entscheidende Rolle in Hinblick auf die Energieverteilung der Ionen im Plasma. Des Weiteren ist zu beachten, dass die Verteilung der Teilchen im Plasma und ihre kinetische Energie winkelabhängig ist. Senkrecht zur Targetoberfläche sind maximale kinetische Energie und Abscheidungsrate an höchsten, für größer werdende Winkel abweichend von der Normalen nehmen beide Größen ab. Grundsätzlich lässt sich die Teilchendichte $D$ als Funktion des Winkels $\Theta$ durch

$$
D(\Theta)=A \cos ^{p} \Theta
$$

beschreiben. Dabei ist $A$ eine von der Laserleistungsdichte abhängige Proportionalitätskonstante. Der Exponent $p$ hängt von Form und Größe des Laserstrahlflecks auf dem Target ab. Mit größeren Fleckdurchmessern vergrößert sich auch $p$ [91, 92]. Die Abhängigkeiten von maximaler kinetischer Energie der Ionen im Plasma und der Abscheidungsrate vom Winkel $\Theta$ bei der PLD von Silber sind in Abbildung 2.21 dargestellt. Auch hier haben die kinetischen Energien der Ionen für Nanosekunden-Pulse typische Werte im Bereich von 10-100 eV.

\subsubsection{Reaktive PLD}

Unter reaktiver PLD soll hier im Folgenden verstanden werden, dass die während der Deposition in der Kammer befindliche Hintergrundgasatmosphäre eine chemische Reaktion mit dem ablatierten Targetmaterial eingeht. Als reaktive Hintergrundgase werden dabei vorwiegend Sauerstoff, Stickstoff, Ammoniak sowie Kohlenwasserstoffe wie Methan und Ethin verwendet. Tabelle 2.3 gibt eine Auswahl an Verbindungen an, die mit Hilfe der reaktiven PLD synthetisiert wurden. 

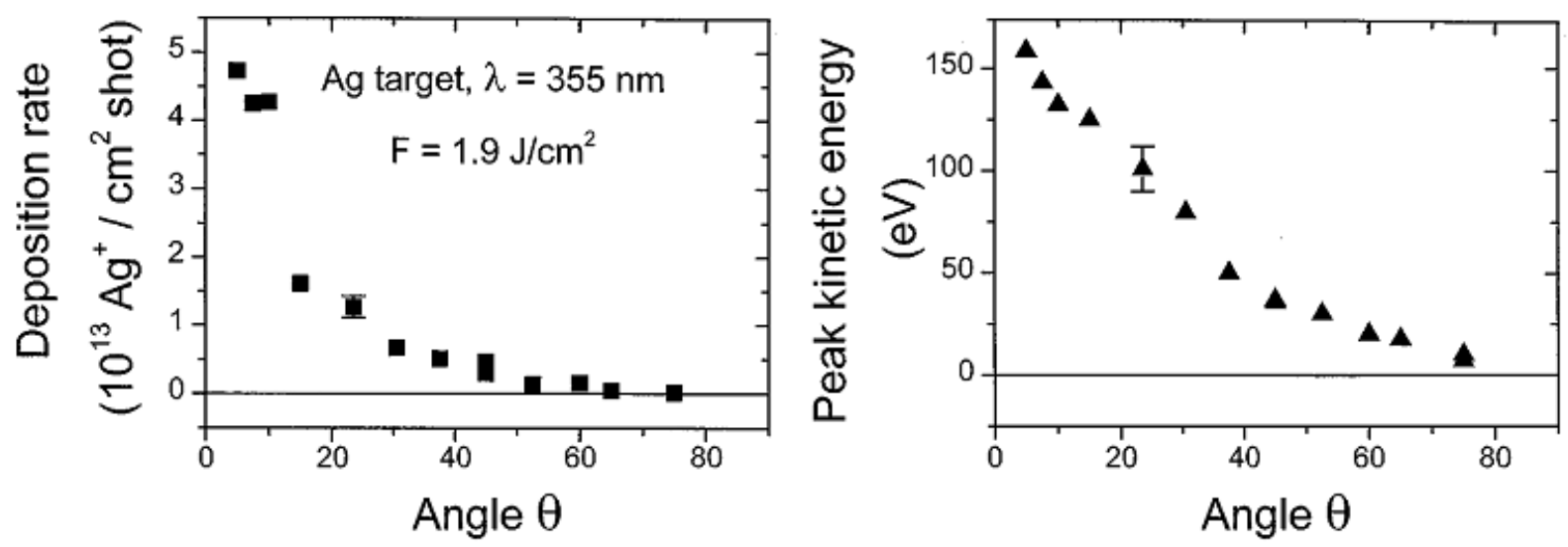

Abbildung 2.21: Winkelabhängige Verteilung der Abscheidungsrate (links) und der maximalen kinetischen Energie der Ionen (rechts) bei der PLD von Silber. $\lambda=355 \mathrm{~nm}$, Pulslänge $\tau=6 \mathrm{~ns}$ [93].

Tabelle 2.3: Eine Auswahl verschiedener Materialklassen und Verbindungen, die mit reaktiver Pulslaserdeposition hergestellt wurden.

\begin{tabular}{|c|c|c|}
\hline Materialklasse & Verbindungen & Referenz \\
\hline Hartwerkstoffe & Siliziumcarbid $\left(\mathrm{SiC}_{x}\right)$ & {$[94,95]$} \\
& Titancarbid $\left(\mathrm{TiC}_{x}\right)$ & {$[96]$} \\
& Kohlenstoffnitrid $\left(\mathrm{CN}_{x}\right)$ & {$[97-100]$} \\
& Siliziumnitrid $\left(\mathrm{SiN}_{x}\right)$ & {$[95,101]$} \\
& Titannitrid $\left(\mathrm{TiN}_{x}\right)$ & {$[102-104]$} \\
III-V-Halbleiter & Aluminiumnitrid $(\mathrm{AlN})$ & {$[105]$} \\
Verschleißschutzschichten & Titan-Aluminium-Nitrid & {$[47]$} \\
& $($ TiAlN $)$ & {$[106]$} \\
& Titan-Aluminium- & \\
Hochtemperatursupraleiter & Carbonitrid & {$[107]$} \\
& $\left((\mathrm{Ti}, \mathrm{Al}) \mathrm{C}_{x} \mathrm{~N}_{1-x}\right)$ & {$[108]$} \\
\hline
\end{tabular}

Untersuchungen zu den Reaktionsmechanismen während der reaktiven PLD wurden in größerem Umfang durchgeführt. Dabei ist von Interesse, wo genau die chemischen Reaktionen stattfinden - in der Schmelze auf dem Target, im Laserplasma bzw. der Gasphase oder erst auf dem Substrat. Bei der Herstellung von $\mathrm{CN}_{x}$-Schichten durch die Ablation von Kohlenstoff in Stickstoff oder Ammoniak ist die Entstehung von CN-Molekülspezies für eine erfolgreiche Synthese notwendig. Massen- und emissionsspektroskopische Methoden haben dabei ergeben, dass diese CN-Moleküle in der Gasphase entstehen. Dies ist in 
Abbildung 2.22 gezeigt. Dargestellt sind die optischen Emissionsintensitäten von CN und $\mathrm{C}_{2}$ entlang der Plasmaachse als Funktion des Abstands zum Graphit-Ablationstarget. Es ist deutlich zu erkennen, dass das benötigte $\mathrm{CN}$ erst in einigem Abstand vom Target in der Gasphase gebildet wird.

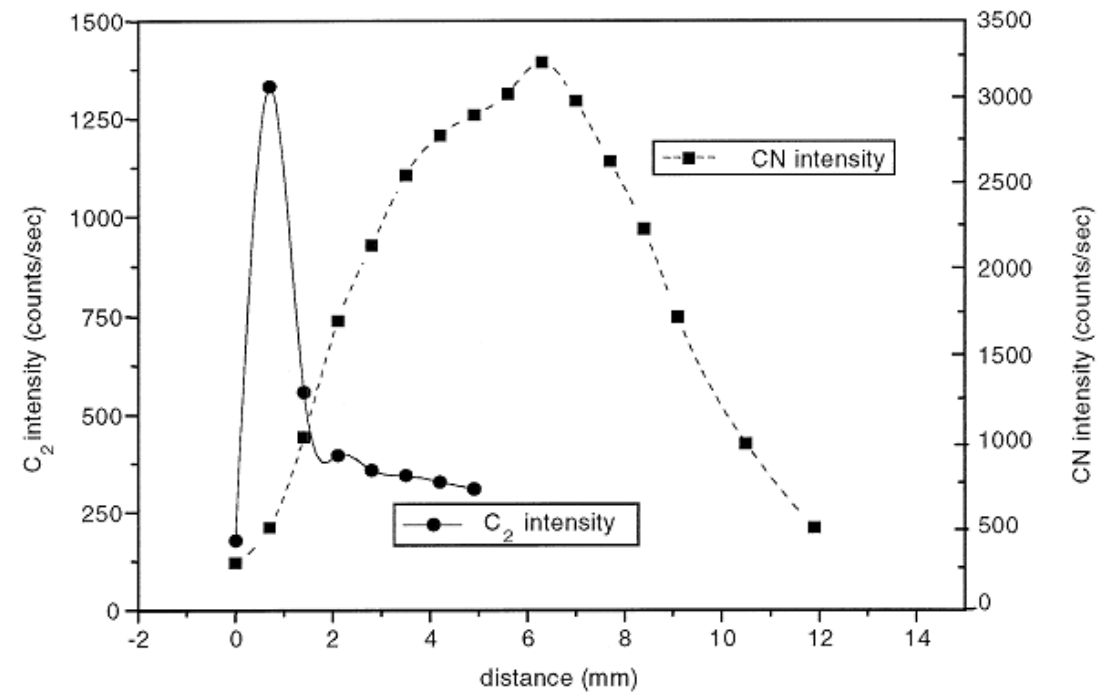

Abbildung 2.22: Verlauf der optischen Emissionsintensitäten von $\mathrm{CN}$ und $\mathrm{C}_{2}$ entlang der Plasmaachse bei der reaktiven PLD von Graphit in Stickstoffatmosphäre [109].

Ähnliches gilt für die reaktive PLD von Aluminiumnitrid. Bei der Ablation von Al in einer Ammoniak $\left(\mathrm{NH}_{3}\right)$-Atmosphäre beobachteten Giardini-Guidoni et al. die Entstehung von $\mathrm{Al}\left(\mathrm{NH}_{3}\right)_{x}$-Clustern in der Gasphase [105]. Sie schlugen vor, dass diese Cluster durch Stoßprozesse auf dem Substrat zerfallen und dadurch AlN-Moleküle gebildet werden. Bei der Ablation von Titan in Stickstoff konnten dagegen keine TiN-Spezies im Plasma nachgewiesen werden [110]. Es ist zwar bekannt, dass Titan bei der Laserbestrahlung in Stickstoffatmosphäre zu TiN reagiert [111, 112]. Allerdings wurden dabei sehr viel größere $\mathrm{N}_{2}$-Hintergrunddrücke verwendet (1 bar und mehr) als bei der Pulslaserdeposition. Bei geringeren $\mathrm{N}_{2}$-Drücken konnten Teodorescu et al. nur Spuren von TiN auf dem Ablationstarget beobachten [113]. Des Weiteren stellten Hermann et al. fest, dass vom Target ablatierte TiN-Moleküle aufgrund der hohen Plasmatemperatur während der Deposition wieder dissoziieren [110]. Diese Ergebnisse deuten darauf hin, dass das Titannitrid außerhalb des Plasmas bzw. erst auf dem Substrat entsteht. Gu et al. stellten dazu fest, dass ein bestimmter Depositionsparameter - nämlich das Produkt aus $\mathrm{N}_{2}$-Druck und Abstand zwischen Target und Substrat - die Güte und Reinheit der TiN-Filme beeinflusst [104]. Eine umfangreiche Untersuchung über einen weiten $\mathrm{N}_{2}$-Druckbereich konnte außerdem zeigen, dass Kristallinität und Reinheit der TiN-Filme stark vom Stickstoffdruck abhängt [102]. Bei geringen $\mathrm{N}_{2}$-Drücken entstehen lediglich geringe Mengen von amorphem TiN. Bei Erhöhung des Stickstoffdrucks werden die Filme zunehmend kristal- 
liner. Ist der $\mathrm{N}_{2}$-Druck jedoch zu hoch, beginnt eine teilweise Oxidation zu TiO und $\mathrm{TiO}_{2}$, welche wiederum eine Kristallisation in den Filmen stark behindert.

\subsection{4 lonenstrahlunterstützte PLD}

Bei der ionenstrahlunterstützten Pulslaserdeposition (engl.: ion beam assisted pulsed laser deposition, IBA-PLD) wird während der Laserablation ein Ionenstrahl auf das Substrat gerichtet, um dadurch auf das Aufwachsen des Filmes und damit seinen Eigenschaften gezielt Einfluss zu nehmen. Grundsätzlich kann man zwischen zwei Arten der IBA-PLD unterscheiden: einerseits kann ein inertes Gas (z.B. Ar) mit entsprechenden Ionenenergien verwendet werden, um die Deposition von Schichten in der chemischen Zusammensetzung des Ablationstargets zu beeinflussen. Auf der anderen Seite kann auch ein reaktives Gas verwendet und dadurch eine verbesserte reaktive PLD durchgeführt werden. Während der konventionellen reaktiven PLD laufen die chemischen Reaktionen nur über relativ unkontrollierte Plasma- und Oberflächenprozesse ab. Bei der IBA-PLD dagegen wird des reaktive Gas direkt auf die Substratoberfläche gerichtet, und es lassen sich mit Gesamtionenstrom und vor allem der Beschleunigungsspannung zwei weitere kontrollierbare experimentelle Größen einführen. Verschiedene Materialien, die mit IBA-PLD deponiert wurden, sind mit den entsprechenden Parametern in Tabelle 2.4 aufgeführt.

Der Vorteil der IBA-PLD liegt darin, dass man mit ihr offensichtlich eine gute Kontrolle über die kristallographischen Eigenschaften der deponierten Schichten hat. Außerdem gibt es Hinweise darauf, dass die hochenergetischen Ionen des reaktiven Gases besser chemisch in den Schichten gebunden werden als bei der konventionellen reaktiven PLD. Dabei ist allerdings zu beachten, dass der Ionenbeschuss während der Deposition auch zu einem Absputtern der Schicht beiträgt, und es deswegen eine obere Energiegrenze für die Ionen gibt, ab der kein Schichtwachstum mehr stattfindet. Bei der IBA-PLD von Bornitrid (BN) beobachteten Angleraud et al. Ionenenergieschwellen bei der die kristalline Phase in der Schicht von hexagonalem h-BN ( $\mathrm{sp}^{2}$-Hybridisierung) in überwiegend kubisches cBN ( $\mathrm{sp}^{3}$-Hybridisierung) übergeht [114]. Diese Schwellenenergie ist dabei vom Verhältnis zwischen ablatierten Material und Zahl der eingestrahlten Ionen abhängig. Abbildung 2.23 stellt dieses Übergangsverhalten von h-BN zu c-BN dar. Bei diesen Untersuchungen wurde weiterhin festgestellt, dass das molare Verhältnis von Stickstoff zu Bor (N/B) bei der IBA-PLD mit Stickstoff $(B / N=0,91)$ größer ist als bei der PLD in Stickstoff ohne Ionenstrahl $(B / N=0,80)$.

Vergleichbare Ergebnisse wurden bei der IBA-PLD von Graphit mit einem StickstoffIonenstrahl von Zhao et al. beschrieben [115]. In den synthetisierten $\mathrm{C}_{3} \mathrm{~N}_{4}$-Schichten sind sowohl das Anteilverhältnis von $\alpha-\mathrm{C}_{3} \mathrm{~N}_{4}$ zu $\beta-\mathrm{C}_{3} \mathrm{~N}_{4}$ (sp ${ }^{3}$-hybridisierte C-Bindungen) als auch der Gesamtstickstoffgehalt von der Energie der eingestrahlten Ionen abhängig. 
Tabelle 2.4: Eine Auswahl verschiedener Materialklassen und Verbindungen, die mit ionenstrahlunterstützter Pulslaserdeposition hergestellt wurden.

\begin{tabular}{|c|c|c|c|}
\hline Materialklasse & Verbindungen & $\begin{array}{c}\text { Hintergrundgas } \\
\text { und } \\
\text { Beschleunigungs- } \\
\text { energien }\end{array}$ & Referenz \\
\hline \multirow[t]{3}{*}{ Hartwerkstoffe } & $\begin{array}{l}\text { kubisches Bornitrid } \\
\left(\mathrm{c}-\mathrm{BN}_{x}\right)\end{array}$ & $\mathrm{Ar} / \mathrm{N}_{2}, 100-1500 \mathrm{eV}$ & {$[114]$} \\
\hline & $\begin{array}{l}\text { Kohlenstoffnitrid } \\
\qquad\left(\mathrm{CN}_{x}\right)\end{array}$ & $\mathrm{N}_{2}, 50-500 \mathrm{eV}$ & {$[115]$} \\
\hline & Titannitrid (TiN) & $\mathrm{N}_{2}, 400-800 \mathrm{eV}$ & [116-118] \\
\hline \multirow[t]{2}{*}{ III-V-Halbleiter } & $\begin{array}{c}\text { kubisches } \\
\text { Aluminiumnitrid } \\
\text { (c-AlN })\end{array}$ & $\mathrm{N}_{2}, 100-400 \mathrm{eV}$ & {$[119]$} \\
\hline & $\begin{array}{l}\text { Aluminiumnitrid } \\
\text { (AlN) }\end{array}$ & $\mathrm{N}_{2}, 200-800 \mathrm{eV}$ & {$[120]$} \\
\hline \multirow[t]{3}{*}{$\begin{array}{l}\text { Pufferschichten für } \\
\text { YBCO }\end{array}$} & $\begin{array}{l}\text { Magnesiumoxid } \\
(\mathrm{MgO})\end{array}$ & $\mathrm{Ar} / \mathrm{O}_{2}, 300-1000 \mathrm{eV}$ & {$[117,121,122]$} \\
\hline & $\begin{array}{c}\text { Yttriumstabilisiertes } \\
\text { Zirkonoxid, YSZ } \\
\left(\mathrm{Y}_{2} \mathrm{O}_{3}: \mathrm{ZrO}_{2}\right)\end{array}$ & Ar, $250 \mathrm{eV}$ & {$[123]$} \\
\hline & $\begin{array}{l}\text { Praseodymoxid } \\
\left(\mathrm{Pr}_{6} \mathrm{O}_{11}\right)\end{array}$ & $\mathrm{Ar} / \mathrm{O}_{2}, 300 \mathrm{eV}$ & {$[124]$} \\
\hline $\begin{array}{l}\text { Hochtemperatur- } \\
\text { supraleiter }\end{array}$ & Niobnitrid (NbN) & $\mathrm{Ar} / \mathrm{N}_{2}, 800 \mathrm{eV}$ & {$[118]$} \\
\hline
\end{tabular}

Mit Hilfe der IBA-PLD gelang Ren et al. die Synthese von Schichten aus kubischem Aluminiumnitrid (c-AlN) mit hoher kristalliner Qualität auf Silizium bereits bei Raumtemperatur. Dazu wurde während der Deposition ein Stickstoffionenstrahl mit Energien bis $400 \mathrm{eV}$ [119] verwendet. Die Kristallinität der Schichten nahm dabei mit zunehmender Ionenenergie zu. Außerdem stieg wiederum der Stickstoffgehalt der Proben mit der Beschleunigungsenergie an. Bei Energien größer als $400 \mathrm{eV}$ war allerdings wiederum das Wachstum der Schichten durch Sputtereffekte stark gehemmt. Six et al. untersuchten bei der IBA-PLD von Aluminiumnitrid mit Stickstoff bzw. Argon den Einfluss des Ionenbeschusses auf die Ausbildung von Spannungen in den Schichten bei einer Depositionstemperatur von $700{ }^{\circ} \mathrm{C}[125,126]$. Sie stellten fest, dass durch den Ionenbeschuss in Abhängigkeit der Ionenenergie und des Einstrahlwinkels die Gitterkonstanten von AlN erheblich verändert werden können. So wurde die kristallografische $c$-Achse von AlN um bis zu 1,8\% verlängert, während die $a$-Achse um bis zu 0,6 \% verkürzt wurde. Bei ei- 


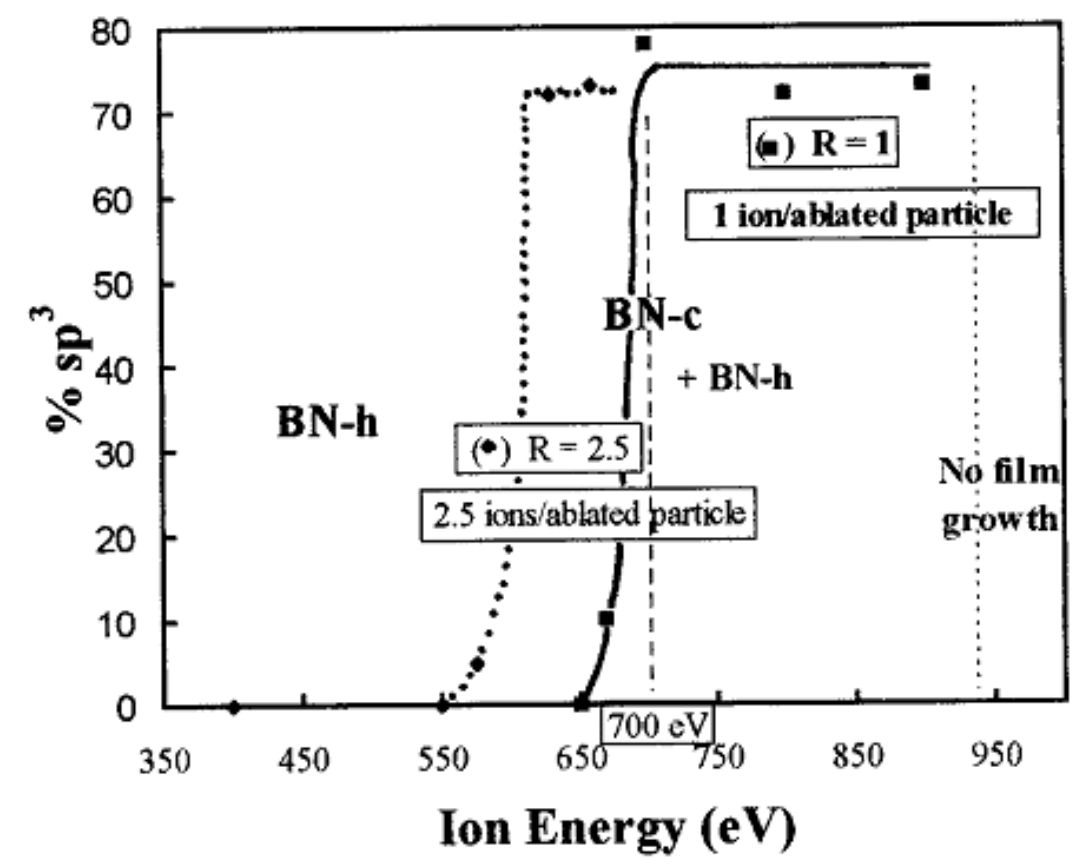

Abbildung 2.23: Anteil der $\mathrm{sp}^{3}$-Bindungen in Bornitrid in Abhängigkeit der Ionenenergie bei der IBA-PLD von BN. Dargestellt sind zwei Kurven verschiedene Verhältnisse von Ionen zu ablatierten Teilchen. Außerdem zu sehen ist die Grenzenergie (ca. $950 \mathrm{eV}$ ), ab der kein Filmwachstum mehr stattfindet [114].

nem Einstrahlwinkel von $30^{\circ}$ zur Substratoberfläche lag das Maximum dieser Gitterparameterverzerrungen bei einer Beschleunigungsenergie von $E_{\text {Ion }}=500 \mathrm{eV}$. Bei Energien größer als 500 eV nahm die kristalline Qualität der Filme wieder stark ab. In Abbildung 2.24 sind die Verschiebungen des AlN(0002)-Röntgenbeugungsreflexes und die prozentuale Verlängerung der AlN-c-Achse durch die Ionenbestrahlung gezeigt.

$\mathrm{Zu}$ beachten ist dabei, dass die Verzerrung der $c$-Achse bei höheren Energien wieder abnimmt. Zur Erklärung dieses Effektes wurde ein einfaches Modell erstellt. Dieses geht davon aus, dass die Gitterverzerrung durch ionenstrahlinduzierte Punktfehler im Gitter - Leerstellen und Zwischengitteratome - hervorgerufen wird. Diese Gitterfehler sind recht mobil und diffundieren in Richtung der Schichtoberfläche. Ihre durchschnittliche Eindringtiefe $\lambda$ ist dabei proportional zur Quadratwurzel der Ionenenergie: $\lambda \propto \sqrt{E_{\text {ion }}}$. Die Dicke der abgesputterten Oberflächenschicht $\xi$ dagegen ist direkt proportional zu $E_{\text {ion }}: \xi \propto E_{\text {ion }}$. Beide Größen sind außerdem winkelabhängig, wobei für kleine Winkel vorwiegend Sputtern auftritt. Die Verzerrungen sind dann eine Funktion der aus Defekterzeugung und Absputtern verbleibenden Defektkonzentration. Aus diesen Überlegungen lässt sich das Maximum der Verzerrungen für eine bestimmte Energie und seine Winkelabhängigkeit leicht erklären.

Eine weitere Anwendungsmöglichkeit der IBA-PLD ist die gezielte Texturierung von 

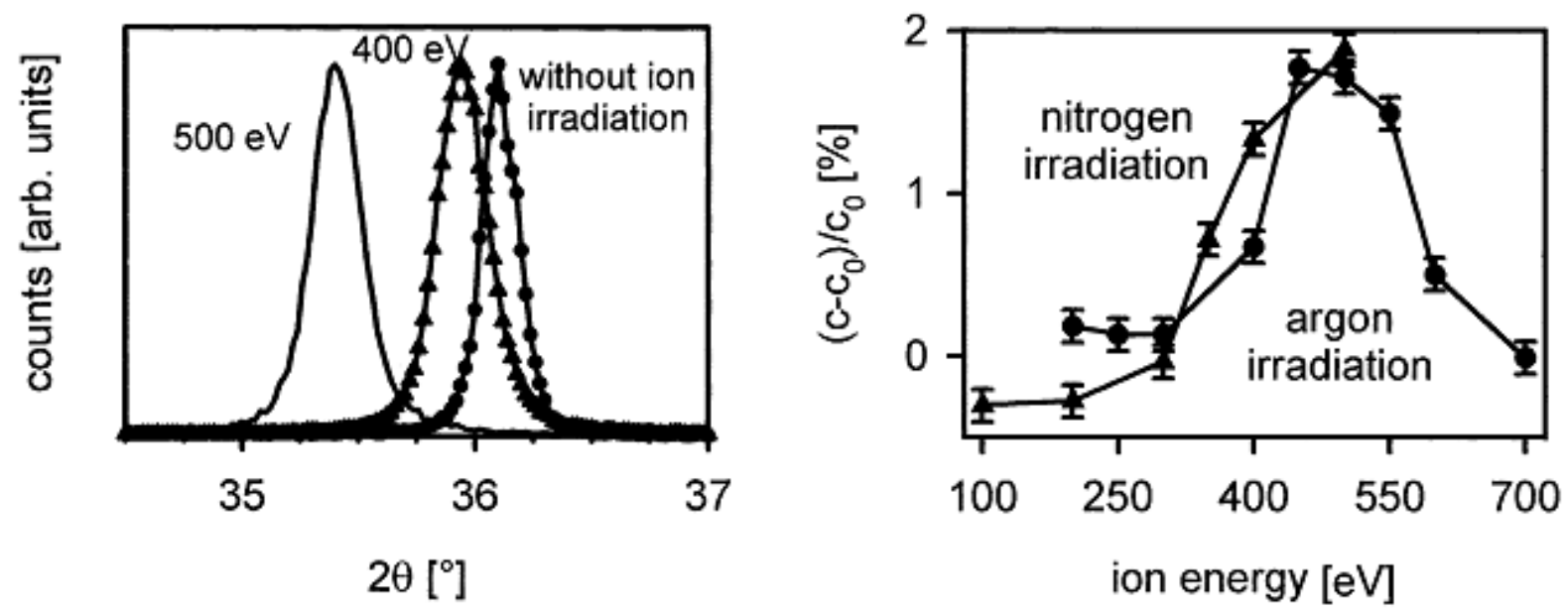

Abbildung 2.24: Verschiebung des $\operatorname{AlN}(0002)$-Reflexes (links) und prozentuale Verlängerug der AlN-c-Achse durch Ionenbeschuss bei der IBA-PLD von AlN [126].

Schichten bei der Deposition. Will man zum Beispiel Hochtemperatursupraleiter wie $\mathrm{YBa}_{2} \mathrm{Cu}_{3} \mathrm{O}_{7-x}$ (YBCO) in größeren Maßstäben anwenden (Hochfeldmagnete oder Übertragungskabel) braucht man YBCO mit entsprechend hohen kritischen Stromdichten. Bei der Deposition auf kristalline Substrate wurde dies bereits erreicht [127]. Auf polykristallinen Metallsubstraten haben die YBCO-Schichten dagegen sehr viele Korngrenzen, die die kritische Stromdichte stark reduzieren. Deswegen ist es notwendig, vororientierte Pufferschichten abzuscheiden, auf denen dann die YBCO-Filme mit der gewünschten Orientierung aufgewachsen werden können. Mögliche Verbindungen für diese Pufferschichten sind, neben yttriumstabilisiertem Zirkonoxid (YSZ), Ceroxid $\mathrm{CeO}_{2}$ und Praseodymoxid $\operatorname{Pr}_{6} \mathrm{O}_{11}$, Magnesiumoxid $(\mathrm{MgO})$ und - wegen seiner relativ hohen elektrischen Leitfähigkeit - Titannitrid (TiN). Hühne et al. gelang die Herstellung solcher dünnen, biaxial texturierten MgO- und TiN-Schichten mit Hilfe der IBA-PLD [116-118, 121, 122]. Aufgrund der großen strukturellen Ähnlichkeit von $\mathrm{MgO}(\mathrm{Fm} \overline{3} \mathrm{~m}, a=0,421 \mathrm{~nm})$ und $\operatorname{TiN}(\mathrm{Fm} \overline{3} \mathrm{~m}$, $a=0,424 \mathrm{~nm}$ ) sind die beobachteten Texturierungen für beide Verbindungen gleich. Zu Beginn der Deposition (wenige Nanometer Schichtdicke) wurde bei Substrattemperaturen oberhalb $250{ }^{\circ} \mathrm{C}$ eine Textur mit der $<200>$-Richtung der Körner parallel zur Substratnormalen beobachtet. Dieses Wachstum ist thermodynamisch begünstigt, da auf diese Weise die Oberflächenenergie minimiert wird, und tritt auch bei der PLD ohne Ionenstrahl auf [121]. Bei fortgesetztem Schichtwachstum wird der Beitrag der Oberflächenenergie zur Gesamtenergie allerdings zunehmend geringer. Statt dessen wird der Einfluss der hochenergetischen Teilchen aus PLD-Plasma und Ionenstrahl größer. Eine Minimierung der Gesamtenergie tritt nun ein, wenn die Körner mit der $<110>$-Richtung parallel zum Ionenstrahl wachsen, wie es auch für TiN diskutiert wurde [128]. Daraus folgt, dass jetzt die $<111>$ - oder $<110>$-Richtungen parallel zur Substratnormalen liegen. Erklärt werden kann dies durch anisotrope Sputterraten von $\mathrm{MgO}$ (bzw. TiN) beim Ionenbeschuss 
[129]. Körner mit der <110>-Richtung parallel zum Ionenstrahl können in der Schicht wachsen. Andere Richtungen werden bevorzugt abgesputtert, oder ihr Wachstum wird aus energetischen Gründen gehindert. 


\section{Methoden}

\subsection{Röntgendiffraktometrie}

Trifft elektromagnetische Strahlung auf periodische Strukturen in der Größe ihrer Wellenlänge treten Beugungseffekte auf. Atomabstände in Kristallen und Molekülen liegen im Bereich von zehntel Nanometern. Dies entspricht der Wellenlänge von Röntgenlicht mit Photonenenergien von etwa 3 bis $8 \mathrm{keV}$. Bei der Bestrahlung kristalliner Materialien mit Röntgenstrahlung dieser Energien kann man folglich konstruktive und destruktive Interferenz beobachten. Die bei der Röntgendiffraktometrie (engl.: X-ray diffractometry, $X R D)$ in Kristallen gemessenen periodischen Strukturen sind die $\langle$ hkl $\rangle$-Netzebenen mit ihrem Ebenenabstand $d$. Konstruktive Interferenz tritt dabei in Abhängigkeit des Abstandes $d$ bei verschiedenen Beugungswinkeln $\theta$ auf. Die notwendige Bedingung wird durch die Bragg-Gleichung beschrieben:

$$
n \cdot \lambda=2 d \sin \theta
$$

Dabei sind $\lambda$ die Wellenlänge der Röntgenstrahlung, $d$ der Gitterebenenabstand, $\theta$ der Beugungswinkel und $n$ die Beugungsordnung des Reflexes. Aus den verschiedenen Netzebenenabständen lassen sich somit - chemische Informationen über das Probenmaterial vorausgesetzt - recht einfach chemische Phasen in der Probe bestimmen. Anhand der Breite der gemessenen Reflexe und eventuell auftretender Verschiebungen des Beugungswinkels kann man weiterhin Aussagen über Korngrößen bzw. Änderungen der Gitterparameter (durch Stress oder Unter-/Überstöchiometrien) treffen. Röntgendiffraktometrie ist eine seit langem bekannte und etablierte Untersuchungsmethode. Die theoretischen Grundlagen allerdings sind relativ umfangreich und komplex und werden an dieser Stelle nicht weiter vertieft. Eine umfassende und detaillierte Beschreibung der Röntgenbeugungsuntersuchung an dünnen Schichten findet sich in [130]. Hier soll im Folgenden nur auf die beiden in dieser Arbeit verwendeten Geometrien und ihre Unterschiede eingegangen werden.

Eine Standardgeometrie zur Messung dünner Schichten ist die so genannte Bragg-Brentanooder auch $\theta / 2 \theta$-Geometrie (s. Abb. 3.1). Dabei verändern sowohl einfallender Strahl als 
auch die Detektorachse kontinuierlich den Winkel $\theta$ bezüglich der Probenoberfläche, wobei die Winkel jederzeit gleich bleiben: $\theta_{\text {ein }}=\theta_{\text {aus }}$. Der Streuvektor $Q$ (die Winkelhalbierende zwischen einfallendem und ausgehendem Strahl) liegt dabei immer parallel zur Substratnormalen $S$. Gemessen werden die Abstände $d$ der $\langle$ hkl $\rangle$-Ebenen, die entlang des Streuvektors $Q$ liegen - bei $\theta / 2 \theta$-Messungen also alle Netzebenen-Scharen, die parallel zur Probenoberfläche ausgerichtet sind. Mit Hilfe der $\theta / 2 \theta$-Geometrie lassen sich dadurch sowohl polykristalline als auch einkristalline, bzw. epitaktische Schichten sehr gut untersuchen.

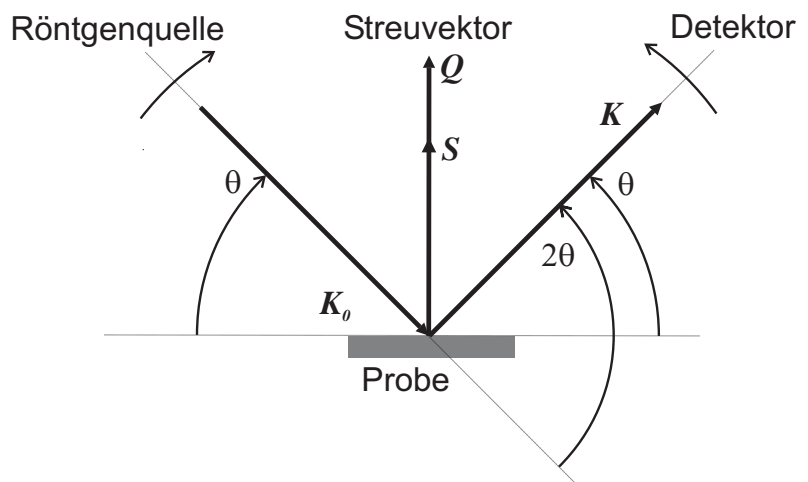

Abbildung 3.1: Strahlengeometrie bei Röntgendiffraktometrie in $\theta / 2 \theta$-Geometrie.

Ein Nachteil der $\theta / 2 \theta$-Geometrie bei der Messung sehr dünner Schichten ist die relativ hohe Eindringtiefe des Röntgenstrahls in das Probenmaterial. Auf diese Weise wird das Messsignal der Schicht stark von Informationen aus dem Substrat überlagert. Um die Eindringtiefe der Röntgenstrahlung in die Probe zu verringern, kann die Probe auch mit streifendem Einfall (engl.: grazing incidence, GI) gemessen werden. In der GI-Geometrie wird ein kleiner $\left(\leq 5^{\circ}\right)$ Winkel $\alpha$ zwischen Röntgenstrahl und Probenoberfläche festgehalten, und nur der Detektor bewegt sich entlang des Winkels $\theta$ (s. Abb. 3.2). Aufgrund des kleinen Einstrahlwinkels werden die Röntgenstrahlen nur von oberflächennahem Material gebeugt und der Anteil der Substratinformation in der Messung folglich verringert.

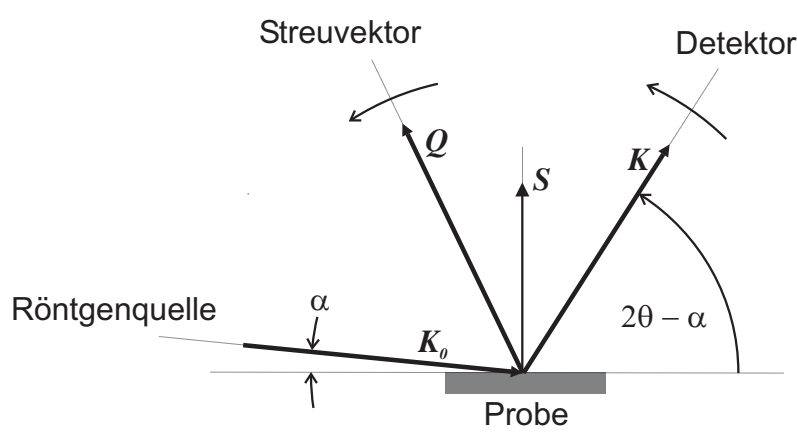

Abbildung 3.2: Strahlengeometrie bei Röntgendiffraktometrie mit streifendem Einfall. 
Allerdings bleibt in der GI-Geometrie der Streuvektor $Q$ nicht konstant parallel zur Substratnormalen $S$. Statt dessen verändert er sich fortwährend mit der Änderung des Detektorwinkels $\theta$. Bei einkristallinen Proben oder epitaktischen Proben mit ausgeprägter Ausrichtung der Netzebenen parallel zur Substratoberfläche lassen sich deswegen keine Beugungsreflexe mehr messen, da $Q$ bei festem $\alpha$ nur für einen Beugungswinkel $\theta$ parallel zu $S$ ist. Dem entsprechend sind nur polykristalline Schichten einer Messung mit streifendem Einfall zugänglich.

Alle Röntgenbeugungsmessungen wurden mit $\mathrm{Cu} \mathrm{K} \mathrm{K}_{\alpha}$-Strahlung $(\lambda=1,5418 \AA)$ am Bruker AXS D8 Advance (Bruker AXS GmbH, Karlsruhe) des II. Physikalischen Instituts der Georg-August-Universität Göttingen, bzw. am Siemens D5000 (Siemens AG, München) des Zentrums für Mikro- und Nanotechnologie der Technischen Universität Ilmenau durchgeführt.

\subsection{Glimmentladungsemissionsspektroskopie}

Bei der Glimmentladungsemissionsspektroskopie (engl.: glow discharge optical emission spectroscopy, GDOES) ermöglicht es die Kombination aus Oberflächenabtragung und Atomemission, sehr schnell Tiefenprofile der chemischen Zusammensetzung von Vollmaterialien, dünnen Schichten oder Grenzschichten zu messen. Dabei kann jede Art von Material gemessen werden.

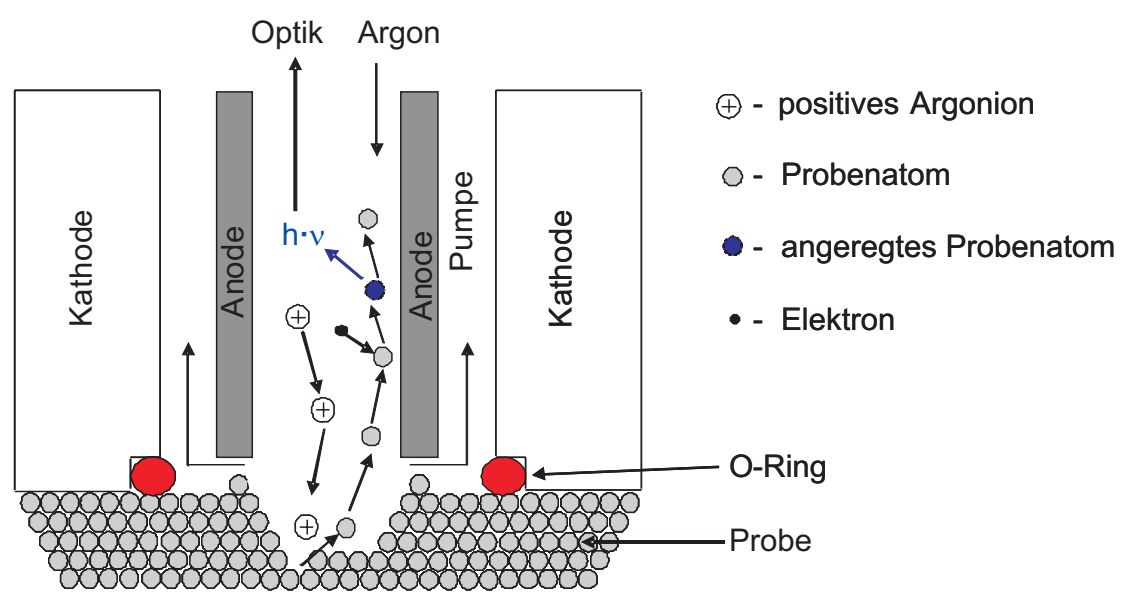

Abbildung 3.3: Prinzipieller Aufbau der GDOES-Hohlanodenquelle. Argongas wird innerhalb der Hohlanode ionisiert und in Richtung Probe beschleunigt. Die aus der Oberfläche geschlagenen Probenatome werden angeregt, und können durch die charakteristische Emissionsstrahlungen gemessen werden. Quelle: Marcus Wilke, TU Ilmenau.

Abbildung 3.3 zeigt die Vorgänge in einer GDOES-Hohlanodenquelle während der Mes- 
sung. Die Anode ist ein hohles Kupferrohr, das nur wenige Zehntelmillimeter oberhalb der Probe, die in diesem Fall als Kathode wirkt, endet. Zunächst wird Argon als Trägergas in den Probenbereich eingeblasen und anschließend eine Spannung zwischen Anode und Kathode aufgebaut. Sind die Betriebsparameter für Ar-Druck und Spannung erreicht, wird das Argon innerhalb der Hohlanode ionisiert und durch den Potentialunterschied in Richtung Probe (=Kathode) beschleunigt. Der geometrische Aufbau der Quelle verhindert dabei Entladungen zwischen Anode und Probe. Durch Optimierung von Spannung, Gasdruck und Ionisationsstrom lässt sich ein schnelles und homogenes Absputtern der Probenoberfläche gewährleisten. Betreibt man die in Abb. 3.3 gezeigte Quelle im Gleichstrommodus, lassen sich selbstverständlich nur elektrisch leitende Proben messen. Es ist allerdings auch möglich, die Glimmentladung über ein Hochfrequenzfeld zu initiieren. Dafür muss die Probe nicht mehr elektrisch leitfähig sein, und alle Materialien werden dadurch für die Messung zugänglich. Die Probenanforderungen für eine GDOES-Messung sind nicht sonderlich hoch. Die Oberfläche muss lediglich glatt genug sein, um die Dichtigkeit am O-Ring zu gewährleisten. Wie bereits erwähnt, laufen die Messungen sehr schnell ab. Übliche Geschwindigkeiten liegen im $n m-\mu \mathrm{m} / \mathrm{s}-$ Bereich. Dabei wird die Elementzusammensetzung der Probe über die Fläche innerhalb der Hohlanode (je nach verwendetem Aufbau 1-8 mm) integriert. Die Tiefenauflösung von GDOES-Messungen liegt unterhalb von $10 \mathrm{~nm}$.

Weil die Proben sinnvollerweise aus mehreren Elementen zusammengesetzt sind, besteht das bei der Emission aus angeregten Zuständen entstehende Licht zwangsläufig aus einer Vielzahl verschiedener Wellenlängen bis hinunter ins ferne Ultraviolett $(\lambda=150 \mathrm{~nm})$. Ein einzelnes Spektrometer ist folglich nicht ausreichend, um alle elementspezifischen Emissionen mit ausreichender Genauigkeit zu messen. Aus diesem Grund wird das Licht aus der Glimmentladung über eine Linse auf ein optisches Gitter geführt, an dem es in seine spektralen Bestandteile zerlegt wird. Die Intensitäten einzelner Wellenlängenbereiche können nun in einer Vielzahl von Photosensoren erfasst werden. In Abbildung 3.4 ist dieser grundsätzliche Aufbau der GDOES-Optik dargestellt. Aus der vorhergehenden Kalibrierung des Gerätes mit Elementstandards lässt sich nun aus den spektrometrischen Ergebnissen eine quantitative Elementanalyse erstellen. Die gemessene Intensität $I$ für eine bestimmte Spektrallinie eines Elements $i$ lässt sich durch

$$
I_{i}=k_{i} \cdot S_{i} \cdot R_{i} \cdot c_{i} \cdot q+b_{i}
$$

beschreiben. Dabei sind

- $k_{i}$ die Detektionseffizienz des Gerätes, abhängig von Transmission durch das Fenster, Effizienz des optischen Gitters, des Photomultipliers und der Detektorelektronik 
- $S_{i}$ der Korrekturterm für die Selbstabsorption; $0<S_{i}<1$, abhängig von der Sputterrate des Elements

- $R_{i}$ die Emissionsausbeute, d.h. die Zahl der Photonen, die von einem gesputtertem Atom im Plasma abgestrahlt werden

$-c_{i}$ die Konzentration des Elements $i$ in der Probe

- $q$ die Gesamtsputterrate und

- $b_{i}$ ein Hintergrundkorrekturterm, der den Dunkelstrom der Photomultiplier, Geräterauschen, gestreutes Licht, Argon-Emission und Überlagerungen von anderen nahen Emissionslinien umfasst.

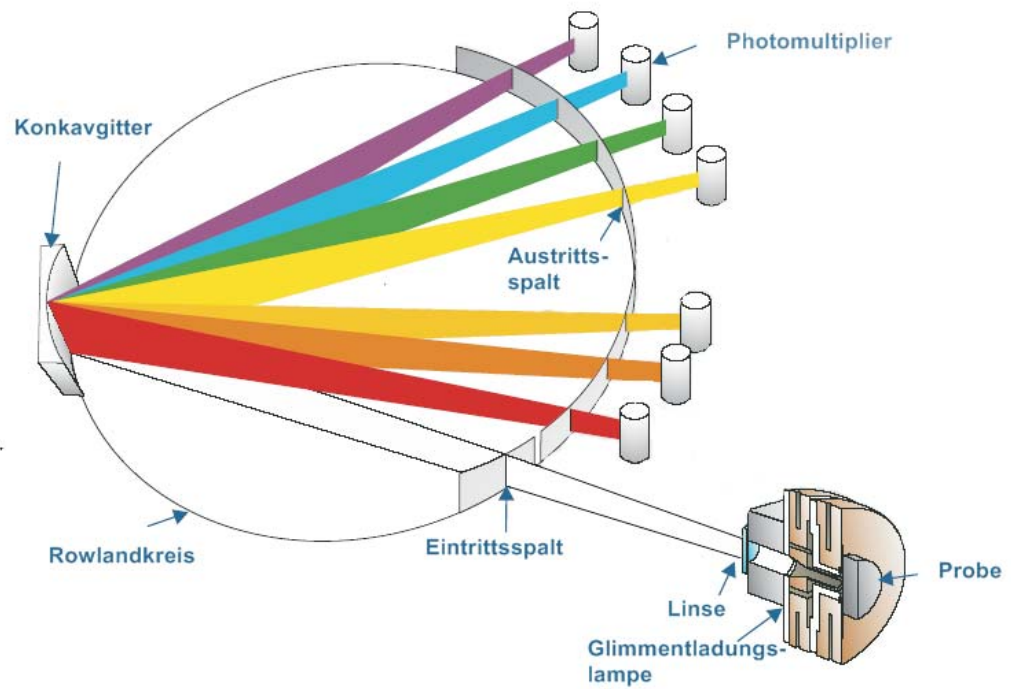

Abbildung 3.4: Schematische Darstellung der Optik eines GDOES-Messgerätes. Das durch Atomemission entstehende Licht wird an einem optischen Gitter zerlegt und über mehrere Photosensoren erfasst. Quelle: LECO Corporation, USA.

Nimmt man $k_{i}, S_{i}, R_{i}$ und $b_{i}$ als konstant an, ändert sich $I_{i}$ linear mit $c_{i} \cdot q$. Gleichzeitig gilt bei einer Analyse, bei der die Signale von allen signifikanten Elementen $i$ aufgenommen werden,

$$
\sum_{i} c_{i}=1
$$

Betrachtet man nun die Gleichungen 3.1 und 3.2 gemeinsam, ergeben sich einerseits die einzelnen Konzentrationen $c_{i}$ aus

$$
c_{i}=\frac{1}{q} \cdot \frac{I_{i}-b_{i}}{k_{i} R_{i} S_{i}}
$$


und andererseits die Gesamtsputterrate $q$ aus

$$
q=\sum_{i} \frac{I_{i}-b_{i}}{k_{i} R_{i} S_{i}}
$$

Die wichtigste Größe in den Gleichungen 3.3 und 3.4 ist die Emissionsausbeute $R_{i}$. Sie hängt auf jeden Fall von den drei experimentellen Parametern Spannung, Strom und Druck ab. Die genauen Zusammenhänge sind immer noch nicht abschließend geklärt und werden deswegen weiterhin eingehend untersucht. Allgemein lässt sich aber sagen, dass wenn Messungen bei den gleichen experimentellen Parametern durchgeführt werden, bei allen Messungen auch $R_{i}$ gleich bleibt. Wichtig ist deswegen die Kalibrierung vor der Messung mit den entsprechenden Standards. Da sich bei der GDOES in der Quelle viele Atome der gleichen Elemente befinden, von denen auch noch ein großer Teil im Grundzustand vorliegt, muss auch das Phänomen der Selbstabsorption beachtet werden. Der entsprechende Korrekturterm kann durch

$$
S_{i} \approx \frac{\exp \left(-s_{S} \cdot c_{i} q\right)}{1+0,412 \cdot s_{E} \cdot c_{i} q}
$$

angenähert werden, wobei $s_{S}$ und $s_{E}$ Konstanten sind [131]. Ist $c_{i} q$, also die Sputterrate des Elementes $i$ klein, dann nähert sich $S_{i} \approx 1$, und die Selbstabsorption kann vernachlässigt werden. Wird aber $c_{i} q$ genügend groß, dann verkleinert sich $S_{i}$, und die Abhängigkeit der gemessenen Intensität $I_{i}$ von der Konzentration $c_{i}$ nach Gl. 3.1 ändert sich signifikant.

Um aus den Gleichungen 3.3 und 3.4 ein quantitatives Tiefenprofil zu gewinnen, ist es nötig, die gemessene Elementkonzentration als Funktion der Schichttiefe darzustellen. Dazu muss der Wert der Gesamtsputterrate $q$ in $[\mu \mathrm{g} / \mathrm{s}]$ - durch geeigenete Annahmen über die Dichte der dünnen Schicht - in eine Eindringrate $w$ in $[\mu \mathrm{m} / \mathrm{s}]$ umgewandelt werden. Danach kann die Eindringrate über die Zeit $t$ integriert werden, um die Schichttiefe $z$ zu erhalten:

$$
z=\sum_{t} w(t) \Delta t
$$

Diese Umwandlung beinhaltet im Wesentlichen drei Schritte. Die gemessenen Rohintensitäten $I_{0}(i, t)$ für alle Elemente $i$ als Funktion der Zeit $t$ werden zunächst mit der relativen Empfindlichkeit bezüglich eines Hauptelements $i_{H}$ auf dieses Hauptelement normalisiert.

$$
I(i, t)=I_{0}(i, t) \cdot \frac{\text { Empfindlichkeit }(\mathrm{i})}{\text { Empfindlichkeit }\left(\mathrm{i}_{\mathrm{H}}\right)}
$$

Die Summe aus den Intensitäten $I(i, t)$ ergibt eine Kurve, die proportional zur Gesamtsputterrate $q$ ist. Nun werden die einzelnen $I(i, t)$ durch diese zu $q$ proportionalen Werte 
geteilt und so normalisiert, dass die Summe aller $I(i, t)$ zu jedem Zeitpunkt $t 100 \%$ ergibt. Man erhält also ein Funktion der Elementkonzentrationen $c_{i}$ (aus der Normalisierung) in Abhängigkeit des Massenverlustes (aus Gesamtsputterrate $q$ und Anteil der jeweiligen Elemente $c_{i}$ ). Durch Betrachtungen zur Dichte der dünnen Schicht (aus elementarer Zusammensetzung und Dichte der einzelnen Elemente) kann nun noch der Massenverlust in eine Tiefe $z$ umgewandelt werden. Das Ergebnis ist dann die gewünschte Tiefeninformation nach der Gleichung

$$
c_{i}=f(z)
$$

Diese Umwandlung wird normalerweise von der Auswertungssoftware des GDOES-Gerätes übernommen. Dabei muss von der Benutzerin allerdings das Hauptelement $i_{H}$ vorgegeben werden.

Neben den oben genannten Vorteilen wie Schnelligkeit und wenig aufwändiger Probenpräparation hat GDOES allerdings auch den Nachteil, nicht zerstörungsfrei zu sein. Misst man die Zusammensetzung einer dünnen Schicht, ist diese nach der Messung am Messort abgesputtert. Zurück bleibt ein kreisrunder Krater mit dem Durchmesser der benutzten Hohlanode. Eine elektronenmikroskopische Aufnahme solch eines Kraters zeigt Abbildung 3.5. In der Aufnahme gut zu erkennen ist die homogene Abtragung des Probenmaterials. Die Aufhäufungen am Kraterrand entstehen durch Materialablagerungen an der Hohlanode während des Sputterprozesses.

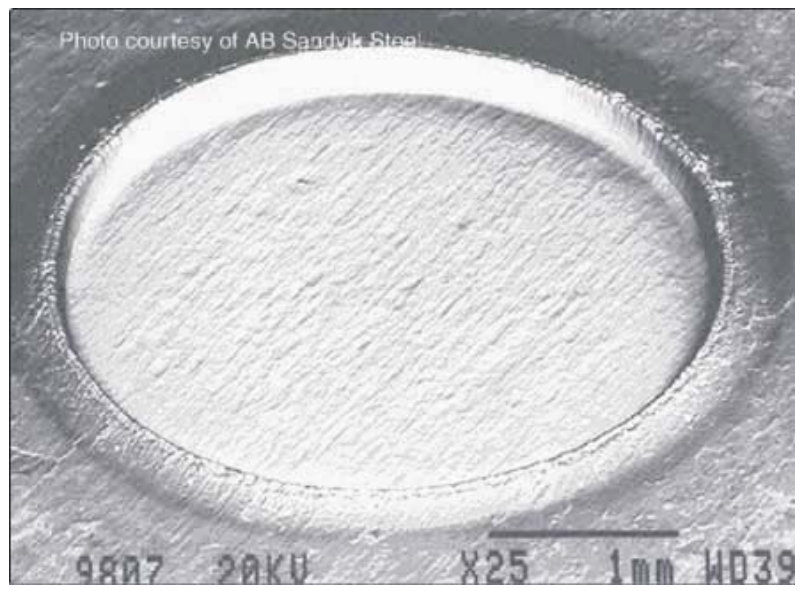

Abbildung 3.5: Elektronenmikroskopische Aufnahme eines GDOES-Messflecks. Gut zu erkennen sind die homogene Abtragung des Probenmaterials und die Ablagerungen am Rand der Hohlanode während des Sputterprozesses. Quelle: LECO Corporation, USA.

Für die GDOES-Analysen in dieser Arbeit wurde das GDA750 Glow Discharge Spectrometer (Spectruma GmbH, Hof) am Institut für Mikro- und Nanotechnologien der Technischen Universität Ilmenau genutzt. 
GDOES ist eine tiefensensitive Messmethode, das heißt die Elementkonzentrationen $c_{i}$ für alle Elemente $i$ in der Schicht werden für jedes Schichtdickenelement $\Delta z$ einzeln gemessen. Zur Angabe des Gesamtgehalts eines Elementes $i$ wurde die Fläche unter der Kurve $y=$ $c_{i}(z)$ numerisch integriert und anschließend durch die Schichtdicke $z$ geteilt. Auf diese Weise erhält man eine mittlere Elementkonzentration $C_{i}$ für jedes Element. Alle totalen Konzentrationsangaben für Schichten aus GDOES-Messungen im Rahmen dieser Arbeit sind diese mittleren Elementkonzentrationen $C_{i}$.

\subsection{Röntgen-Photoelektronenspektroskopie}

Die Photoelektronenspektroskopie mit Röntgenstrahlung (engl.: X-Ray photoelectron spectroscopy, XPS) basiert auf dem Prinzip der Photoemission, bei der Materie durch die Bestrahlung mit elektromagnetischen Wellen (VIS, UV, Röntgen) Elektronen emittiert. Elektronen in der Probe werden durch die Absorption der eingestrahlten Photonen angeregt und können so die Probe verlassen. Ihre kinetische Energie $E_{k i n}$ beim Austritt beträgt

$$
E_{k i n}=\hbar \nu-\phi-\left|E_{B}\right|
$$

Dabei sind $\hbar \nu$ die Energie des absorbierten Photons, $\phi$ die Austrittsarbeit und $E_{B}$ die Bindungsenergie des Elektrons in der Probe. In einem XPS-Experiment (s. Abb. 3.6) werden die emittierten Photoelektronen in einem elektrostatischen Separator entsprechend ihrer kinetischen Energie getrennt und anschließend mit einem Photomultiplier detektiert. Da sowohl $\hbar \nu$ als auch $\phi$ für ein gegebenes Element bekannt sind, lässt sich die Zahl der detektierten Elektronen auch als Funktion der Bindungsenergie $E_{B}$ angeben. Dadurch können die Ergebnisse unabhängig von der verwendeten Anregungsquelle dargestellt werden, und Ergebnisse aus verschiedenen experimentellen Aufbauten werden direkt vergleichbar. Die Werte der gemessenen Bindungsenergien $E_{B}$ liefern dabei Informationen über die elektronische Struktur des Probenmaterials, d.h. Angaben über die chemische Zusammensetzung der Probe und die elektronischen Zustände der untersuchten Elemente. Die genaue Auswertung der gemessenen Spektren erfolgt in aller Regel durch den Vergleich mit entsprechenden Referenzen aus Datenbanken.

Um bei der XPS detektiert zu werden, müssen die Photoelektronen die Probe verlassen können. Aus diesem Grund ist der limitierende Faktor für die Informationstiefe bei der XPS die mittlere freie Weglänge der Elektronen in der Probe. In Abbildung 3.7 sind beispielhaft die mittleren freien Weglängen von Elektronen in Kupfer und Aluminium dargestellt. Abhängig von der kinetischen Energie der Elektronen liegen diese zwischen 5 und $40 \AA$. Das bedeutet, das mit XPS nur sehr oberflächennahe Bereiche der Probe 


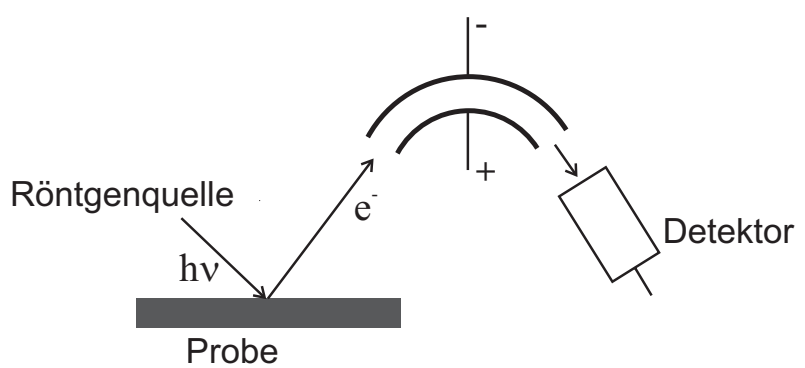

Abbildung 3.6: Schematische Darstellung eines XPS-Experiments. Durch Absorption der Röntgenphotonen angeregte kernnahe Elektronen verlassen die Probe, werden mit einem elektrostatischen Separator getrennt und anschließend mittels eines Photomultipliers detektiert.

gemessen werden können. Für eine sinnvolle Messung von dünnen Schichten müssen also hochreine Oberflächen im Ultrahochvakuum (UHV) präpariert werden. Eine weitere Möglichkeit, tatsächlich Informationen aus der deponierten Schicht zu erhalten ist, vor der XPS-Messung zunächst einige Nanometer der kontaminierten Oberfläche mit einem inerten Ionenstrahl abzusputtern. Bei den Messungen in dieser Arbeit wurde diese Methode angewandt, in dem der zu messende Bereich mit einem $4 \mathrm{kV} \mathrm{Ar}{ }^{+}$-Ionenstrahl auf einer Fläche von $2 \times 2 \mathrm{~mm}^{2}$ für 60 Sekunden vorgesputtert wurde.
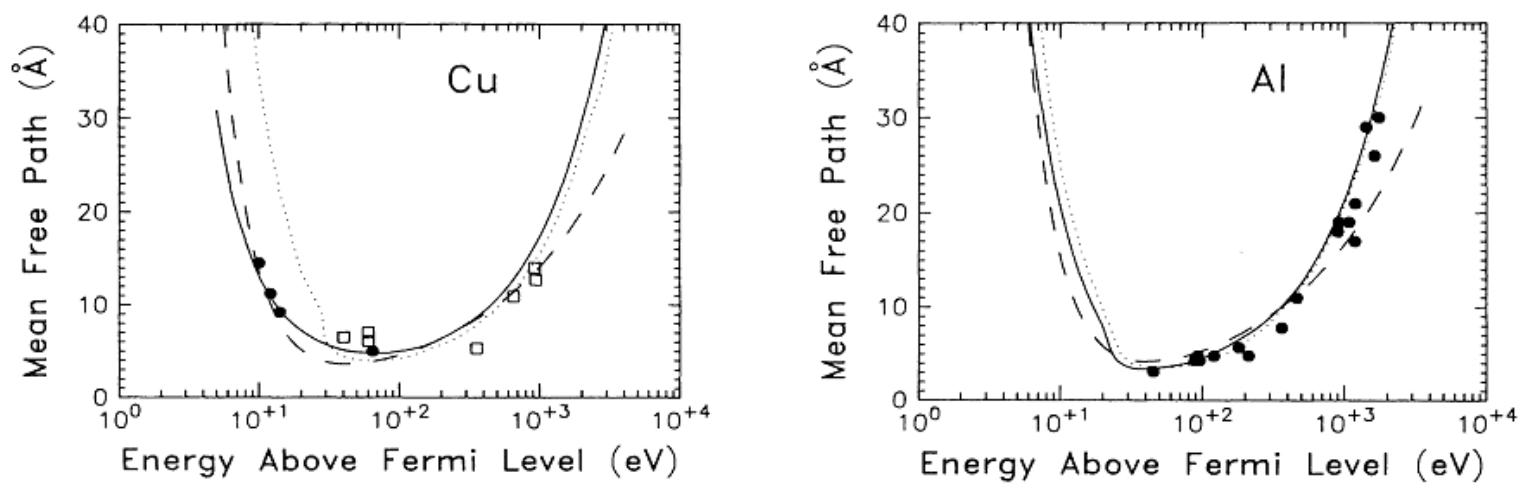

Abbildung 3.7: Mittlere freie Weglängen von Elektronen als Funktion der kinetischen Energie in Kupfer (links) und Aluminium (rechts). Gezeigt sind sowohl theoretisch modellierte Daten (Linien) als auch experimentell bestimmte Werte (Kreise und Quadrate) [132].

Für alle hier gezeigten XPS-Messungen wurde das Physical Electronics (Phi) Quantum 2000 der Abteilung für Materialchemie an der Universität Uppsala, Schweden genutzt. Gemessen wurde mit Al- $\mathrm{K}_{\alpha}$-Strahlung $(\hbar \nu=1487 \mathrm{eV})$ mit einem Messfleckdurchmesser von $150 \mu \mathrm{m}$. 


\subsection{Mikrohärtemessungen}

Die Standardmethode zur Bestimmung der Härte eines Materials ist die Druckbelastung durch einem kleinen, sehr harten (meist Diamant) Prüfkörper mit bekannter Geometrie. Die Härte ist dann als Eindringwiderstand der Probe, also die Eindringtiefe $h$ des Prüfkörpers, gegenüber der Prüfkraft $F$ definiert (Abb. 3.8).

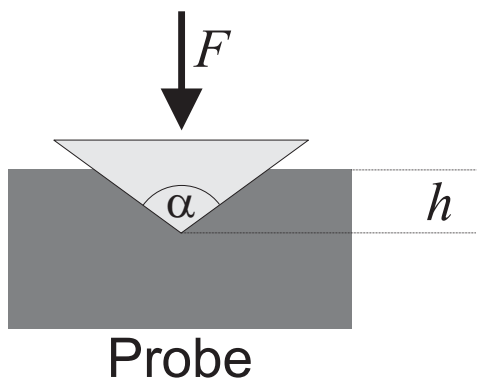

Abbildung 3.8: Schematische Darstellung der Mikrohärtemessung.

Von Mikrohärtemessungen spricht man, wenn die Prüfkraft zwischen 0,01 und $2 \mathrm{~N}$ liegt. Die Martenshärte $H_{M}$ (auch Universalhärte $H_{U}$ ) ist definiert als der Quotient von Prüfkraft $F$ und Eindruckfläche $A(h)$. Im hier vorliegenden Fall ist der Prüfkörper eine quadratische Pyramide aus Diamant mit einem Öffnungswinkel $\alpha$ von $136^{\circ}$. Damit ergibt sich die Martenshärte $H_{M}$ aus folgender Gleichung

$$
H_{M}=\frac{F}{A(h)}=\frac{F}{4 h^{2} \frac{\sin (\alpha / 2)}{\cos (\alpha / 2)}}=\frac{F}{26,43 h^{2}} \quad\left(\alpha=136^{\circ}\right)
$$

Die Anwendung dieser Gleichung setzt eine ideale Geometrie voraus. Da diese im Experiment nicht vorliegt, muss für das Gerät durch die Bestimmung eines Materials bekannter Härte (Silizium) eine Korrektur durchgeführt werden. Detaillierte Beschreibungen dieser Thematik finden sich in [133].

Die Mikrohärtemessungen wurden am Härteprüfgerät Fischerscope H100 des II. Physikalischen Instituts der Georg-August-Universität Göttingen mit einer Prüfkraft von $3 \mathrm{mN}$ durchgeführt. Das Gerät verfügt über einen Mikrokontroller mit Computersteuerung, so dass die Messungen vollautomatisiert ausgeführt werden können. Um Probeninhomogenitäten auszugleichen wurden Felder von 5x5 Prüfpunkten gemessen. 


\subsection{Elektronenmikroskopie}

\subsubsection{Rasterelektronenmikroskopie}

Bei der Rasterelektronenmikroskopie (REM) wird die Probe mit einem hochenergetischen Elektronenstrahl abgerastert. Durch die Interaktion dieser Elektronen mit den Probenatomen können auf diese Weise Informationen zur Probentopographie und zur chemischen Zusammensetzung der Probe gewonnen werden. Die Bildgebung bei REM erfolgt hauptsächlich durch so genannte Sekundärelektronen (SE) oder rückgestreute Elektronen (engl.: backscattered electrons (BSE)). Sekundärelektronen sind Elektronen niedriger Energie $(E<50 \mathrm{eV})$, die aus den K-Schalen der Probenatome durch inelastische Streuung des Elektronenstrahls austreten. Da die Effizienz dieser inelastischen Streuung stark von Einstrahlwinkel des Elektronenstrahls zur Probe abhängt, lässt sich auf diese Weise die Topographie der Probenoberfläche sehr gut darstellen. Abhängig vom verwendeten Gerät und der zugänglichen Beschleunigungsspannung des Elektronenstrahls können Vergrößerungen bis zu 900000:1 erreicht werden. Bei rückgestreuten Elektronen handelt es sich um hochenergetische Elektronen aus dem Elektronenstahl, die von den Atomen in der Probe durch elastische Wechselwirkung reflektiert bzw. rückgestreut werden. Für schwerere Atome (hohe Ordnungszahl) ist der Wirkungsquerschnitt der inelastischen Wechselwirkung sehr viel höher als bei leichten Atomen (niedrige Ordnungszahl). Folglich erscheinen schwere Atome in einer BSE-Aufnahme heller als leichte Atome, d.h. das aufgenommene Bild zeigt Hell/Dunkel-Kontraste zwischen Bereichen unterschiedlicher chemischer Zusammensetzung.

Für die REM-Aufnahmen wurde ein Leo Supra35TM (Carl Zeiss SMT AG, Oberkochen) des Instituts für Materialphysik der Georg-August-Universität Göttingen genutzt. Das Gerät verfügt über eine Gemini-Säule, bestehend aus einer Schottky-Feldemissionskathode $(\mathrm{ZrO} / \mathrm{W})$, Elektronenlinsen und Sekundärelektronen-Ringdetektor (in-lens). Außerdem stehen ein Everhart-Thornley-Sekundärelektronendetektor und ein Quadrant-Rückstreudetektor (QBSD) zur Verfügung.

\subsubsection{Transmissionselektronenmikroskopie}

Das Prinzip der Transmissionselektronenmikroskopie (TEM) ist eng mit dem der konventionellen Durchlichtmikroskopie verwandt. Allerdings werden dabei keine Photonen, sondern hochenergetische Elektronen zur Bildgebung verwendet. Betrachtet man ein Elektron als Materiewelle, so ist seine Wellenlänge $\lambda_{e}$ - abhängig von seinem Impuls $p$ und der Planckkonstanten $h$ - entsprechend der de Broglie-Beziehung 


$$
\lambda_{e}=\frac{h}{p}
$$

gegeben. Die Energie eines Elektrons mit der Ruhemasse $m_{0}$ und der Ladung $e$, das einer Beschleunigungsspannung $U_{B}$ ausgesetzt wird, beträgt

$$
E=\sqrt{p^{2} c^{2}+m_{0}^{2} c^{4}}=m_{0} c^{2}+e \cdot U_{B} \Rightarrow p=\sqrt{2 m_{0} e \cdot U_{B}\left(1+\frac{e \cdot U_{B}}{2 m_{0} c^{2}}\right)}
$$

Setzt man Gl. 3.7 in Gl. 3.6 ein, so erhält man für die Wellenlänge des Elektrons als Funktion der Beschleunigungsspannung

$$
\lambda_{e}=\frac{h}{\sqrt{2 m_{0} e \cdot U_{B}\left(1+\frac{e \cdot U_{B}}{2 m_{0} c^{2}}\right)}} \Rightarrow \lambda_{e}[n m] \approx \sqrt{\frac{1,5}{U_{B}[V]}}
$$

Bei der für TEM typischen Beschleunigungsspannung von $200 \mathrm{kV}$ liegt $\lambda_{e}$ also bei etwa 2,7 pm. Damit liegt das theoretische Auflösungslimit also im Picometerbereich. Durch optische Abbildungsfehler wie spherische und chromatische Abberationen können praktisch Auflösungen von etwa 0,1 bis $0,2 \mathrm{~nm}$ erreicht werden.

Für die TEM-Aufnahmen wurden das FEI TECNAI 20 S-TWIN TEM (FEI Company, Hillsboro, USA) mit CCD-Detektor des Zentrums für Mikro- und Nanotechnologien der TU Ilmenau verwendet. Die Beschleunigungsspannung betrug 200 kV.

\subsection{Messung elektrischer Eigenschaften}

Eine wichtige elektrische Eigenschaft dünner Schichten ist ihr elektrischer Schichtwiderstand $R_{s}$. Aus ihm lassen sich im Vergleich zum spezifischen Widerstand des entsprechenden Vollmaterials Rückschlüsse auf die Eigenschaften des Filmes ziehen. Durchfließt ein Strom $I$ einer Leiter der Querfläche $A$ und der Länge $L$, so gilt für den ohmschen Widerstand $R$ des Leiters

$$
R=\rho \cdot \frac{L}{A}
$$

wobei $\rho$ der spezifische Widerstand des Materials ist. Ersetzt man für eine dünne Schicht den Ausdruck für $A$ durch das Produkt aus Schichtbreite $B$ und Schichtdicke $d$ und führt man gleichzeitig den Schichtwiderstand $R_{s}$ als $\rho / d$ ein, so gilt:

$$
R=\frac{\rho}{d} \frac{L}{B}=R_{s} \frac{L}{B}
$$




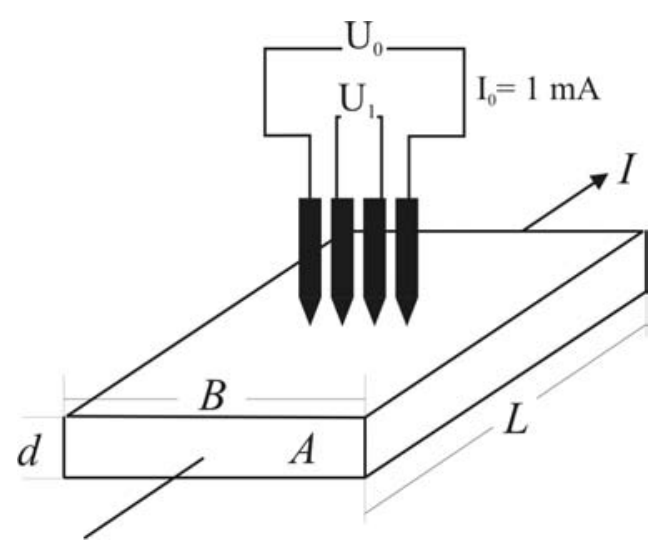

Abbildung 3.9: Bestimmung des Schichtwiderstandes $R_{s}$ einer dünnen Schicht.

Für quadratische Proben mit $L=B$ - wie im Falle dieser Arbeit - ist folglich $R=R_{s}$.

Der Widerstand $R$ einer Schicht lässt sich relativ einfach mit der so genannten VierPunkt-Methode messen. Dabei werden vier Messspitzen mit gleichem Abstand in einer Reihe auf die Probe aufgesetzt. Über die beiden äußeren Spitzen wird ein bekannter Strom $I_{0}$ eingespeist, während zwischen den beiden inneren Spitzen die Potentialdifferenz $U_{1}$ gemessen wird. Durch eine Kompensationsschaltung $\left(I_{1}=0\right)$ wird diese Messung stromlos durchgeführt. Der Flächenwiderstand ergibt sich dann aus $I_{0}$ und $U_{1}$ durch die Gleichung

$$
R_{s}=\frac{\pi}{\ln 2} \frac{U_{1}}{I_{0}}
$$

Zur Messung im Rahmen dieser Arbeit wurde ein Eigenbau-Vier-Punkt-Messgerät des Instituts für Mikro- und Nanotechnologien an der Technischen Universität Ilmenau genutzt. Die eingespeiste Stromstärke $I_{0}$ betrug dabei $I_{0}=1 \mathrm{~mA}$. 


\section{Aufbau der PLD-Anlage}

Die Arbeitsgruppe beschäftigt sich schon seit längerem intensiv mit der Laserbearbeitung von Materialien und Untersuchungen zur Laser-Materie-Wechselwirkungen. Ein Teil der hier vorgelegten Arbeit ist der Aufbau einer Anlage zur Pulslaserdeposition, um entsprechende Forschungen auch auf diesem Gebiet durchführen zu können. Im folgenden Kapitel soll der Aufbau dieses PLD-Experiments detailliert beschrieben werden.

\subsection{Rohbau}

Wie schon in Abschitt 2.2 beschrieben, besteht eine PLD-Anlage im Wesentlichen aus einer Vakuumkammer, in die das Laserlicht eingestrahlt wird, und in der bewegliche Targethalter und heizbare Substrathalter eingebaut sind. Systeme zur Probeneinschleusung und zum kontrollierten Gaseinlass sind ebenfalls notwendig. Außerdem musste in diesem Fall bei der Konstruktion noch der Einbau der Ionenstrahlquelle berücksichtigt werden. Der Grundkörper der Vakuumkammer ist ein Edelstahlzylinder (Durchmesser 400 mm), in den alle benötigten Flansche eingeschweißt wurden. Das Fenster, durch das Laserlicht eintritt, besteht aus Quarzglas (Durchmesser $50 \mathrm{~mm}$, Dicke $10 \mathrm{~mm}$ ), und ist in den Lasereintrittsflansch (CF, Durchmesser $200 \mathrm{~mm}$ ) eingelassen. Abbildung 4.1 zeigt die entsprechende Konstruktionszeichnung mit der Bezeichnung der derzeit genutzten Flansche. Die Zeichnung stellt die Ansicht von unten dar. Da die Probenaufnahme etwa in der Mitte der Kammer positioniert werden muss, und außerdem am Probenhalter elektrische Kontakte für Heizung, Schwingquarz und Gegenspannung angebracht sind, muss die Probenaufnahme entsprechend weit entfernt vom Flansch untergebracht werden, und der Flansch selbst mit den entsprechenden elektrischen Durchführungen versehen werden. 


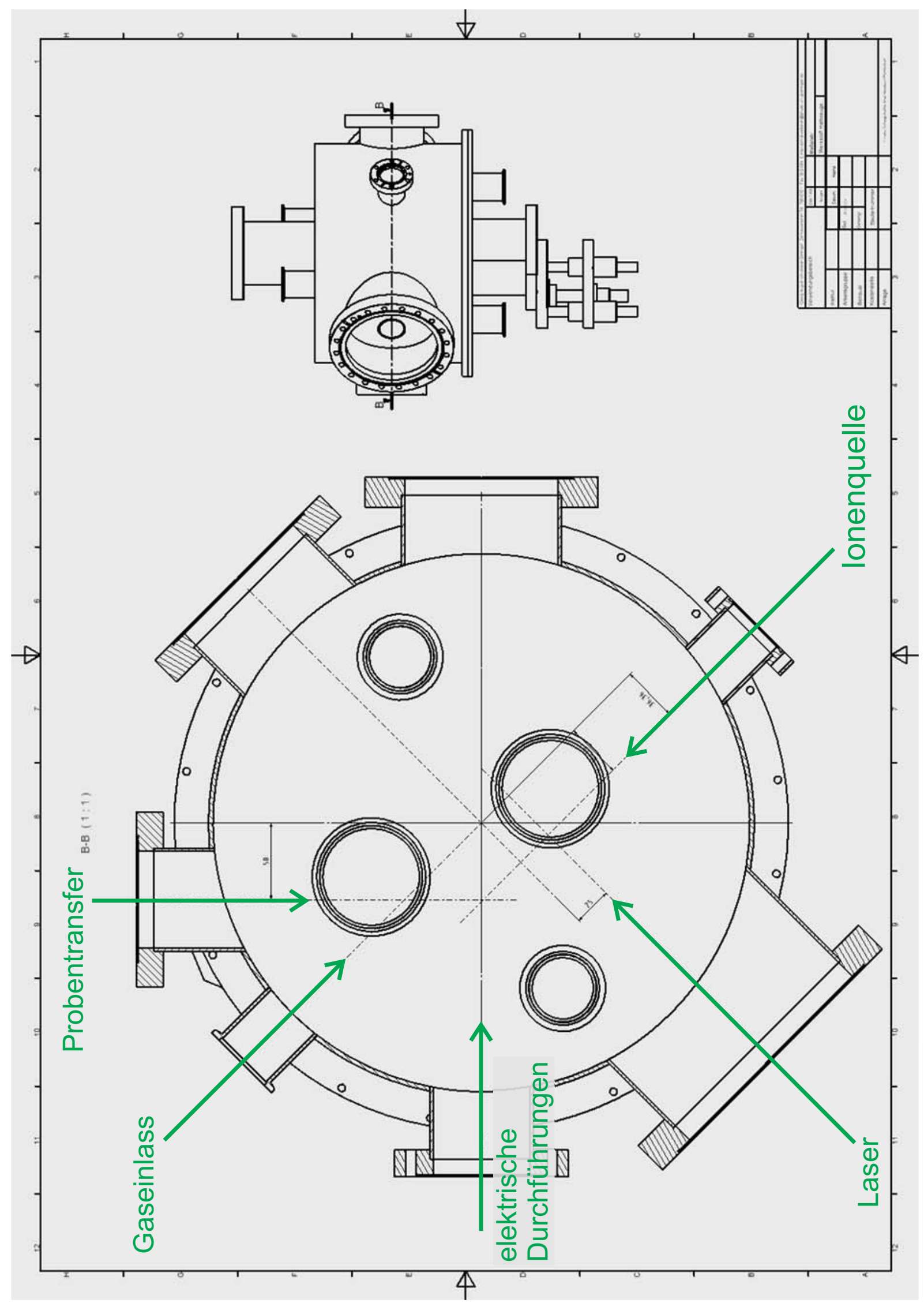

Abbildung 4.1: Konstruktionszeichnung der aufgebauten PLD-Anlage mit Bezeichnung der genutzten Flansche (Ansicht von unten). Zeichnung: Zentralwerkstatt der Fakultät für Physik, Uni Göttingen. 
Das dafür notwendige Konstruktionsteil zeigt die CAD-Zeichnung in Abb. 4.2. Zu sehen ist die eigentliche Probenaufnahme, in die der Probenhalter mit dem Substrat eingeschoben wird. Der rechteckige Halter der Probenaufnahme (grau) ist von der Dreiecksstrebe durch ein 10x10x25 mm³ großes Stück Glaskeramik (Macor ${ }^{\circledR}$, Corning Incorporated, Corning, USA) isoliert (in der Zeichnung nicht zu sehen). Links und rechts von der Dreiecksstrebe sind im Flansch die Öffnungen für die elektrischen Durchführungen zu erkennen. Zur besseren Kontrolle der Depositionen wurde unterhalb der Probenaufnahme zusätzlich ein Schwingquarz angebracht. Die Temperaturkontrolle erfolgt über ein NiCr-Ni (Typ K)Thermoelement, dessen Messspitze in einer entsprechenden Bohrung in dem Kupferbauteil versenkt ist.

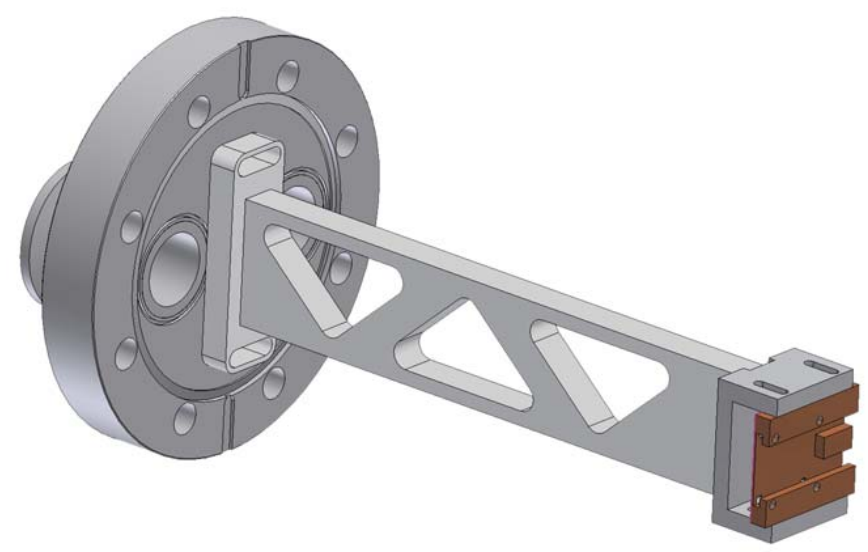

Abbildung 4.2: CAD-Zeichnung des Probenaufnahmeflansches mit Probenaufnahme aus Kupfer (braun) und Öffungen für die elektrischen Durchführungen. (Zeichnung: Zentralwerkstatt der Fakultät für Physik, Uni Göttingen.)

Die Abbildung 4.3 zeigt die Probenaufnahme in der tatsächlich realisierten Form mit Schwingquarz, Heizelement, Thermoelement und allen benötigten Anschlüssen. Dazu gehören die Gleichstromversorgung für das Heizelement, ein elektrischer Anschluss zum Anlegen der Gegenspannung sowie Spannungsversorgung und Kühlwasseranschluss für den Schwingquarz. Der Probentransfer erfolgt über eine Probentransferstange, die aus der Kammer gezogen werden kann. Außerhalb der Kammer wird der Substrathalter in einer Schleuse auf diese Stange geschraubt und nach dem Einschleusen seitlich in die Probenaufnahme eingeschoben.

Der Substrathalter selbst ist eine $2 \mathrm{~mm}$ dicke Platte aus Kupfer (etwa 32x20 mm²), auf die das Substrat unter einen $1 \mathrm{~mm}$ dicken Halterahmen geschraubt wird. Die Fensteröffnung in diesem Rahmen ist quadratisch mit einer Seitenlänge von neun Millimetern. Das heißt, das in der Anlage dünne Schichten mit quadratischer Form der Größe 9x9 mm² deponiert werden können. An einem Ende des Substrathalters befindet sich ein Gewinde M6, mit dem der Substrathalter in die Probentransferstange eingeschraubt wird. Die Konstruktionszeichnung des Substrathalters mit den genauen Maßen ist in Abbildung 4.4 dargestellt. 


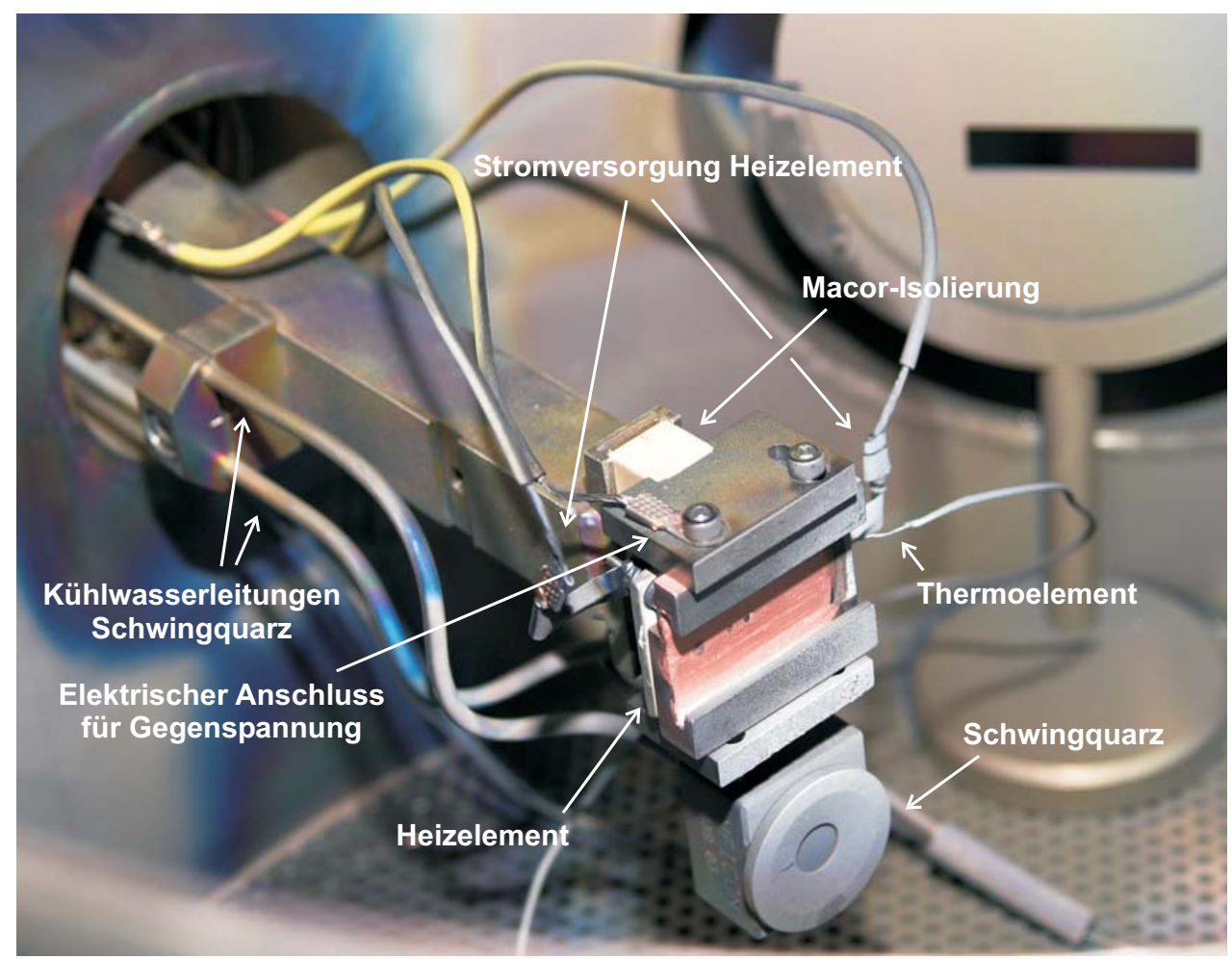

Abbildung 4.3: Tatsächliche Ausführung der Probenaufnahme in der PLD-Kammer mit Thermoelement, Schwingquarz und elektrischen Anschlüssen.

Der Substrathalter ist aus einem Stück gearbeitet, das bedeutet, dass das Gewinde nicht extra gefertigt und eingelötet ist. Die möglichen hohen Temperaturen während der Deposition würden die Lötstelle zerstören.

\subsection{Substratheizung}

Zur Heizung des Substrates dient ein rechteckiges $\left(35 \times 25 \mathrm{~mm}^{2}\right)$ Boralectric ${ }^{\circledR}$-Heizelement (Tectra GmbH, Frankfurt/Main), das direkt von hinten auf die Probenaufnahme geschraubt ist. Dieses Heizelement besteht aus pyrolytischem Bornitrid $(p \mathrm{BN})$, in dessen Innerem elektrische Leiterbahnen aus Graphit eingelassen sind, mit denen sich das Element als Widerstandsheizung betreiben lässt. An zwei Ecken des Heizelements sind offene Graphitkontaktstellen, die mit Tantalgewindestiften und Graphitunterlegscheiben elektrisch kontaktiert werden (s. Abb. 4.5). Die maximale Heizstromstärke beträgt $8 \mathrm{~A}$, die maximale Heiztemperatur liegt bei etwa $1000{ }^{\circ} \mathrm{C}$. Bei Temperaturen über $1000{ }^{\circ} \mathrm{C}$ besteht die Gefahr, das Heizelement zu beschädigen. Insbesondere die Graphitkontakte können dann durch Restsauerstoff im Vakuum angegriffen werden. Ein Betrieb ohne vorheriges Abpumpen der Vakuumkammer schließt sich folglich aus. Da die den Heizer umgebenden Bauteile aus Kupfer (Schmelztemperatur $1084{ }^{\circ} \mathrm{C}$ ) gefertigt sind, empfiehlt es sich, die 


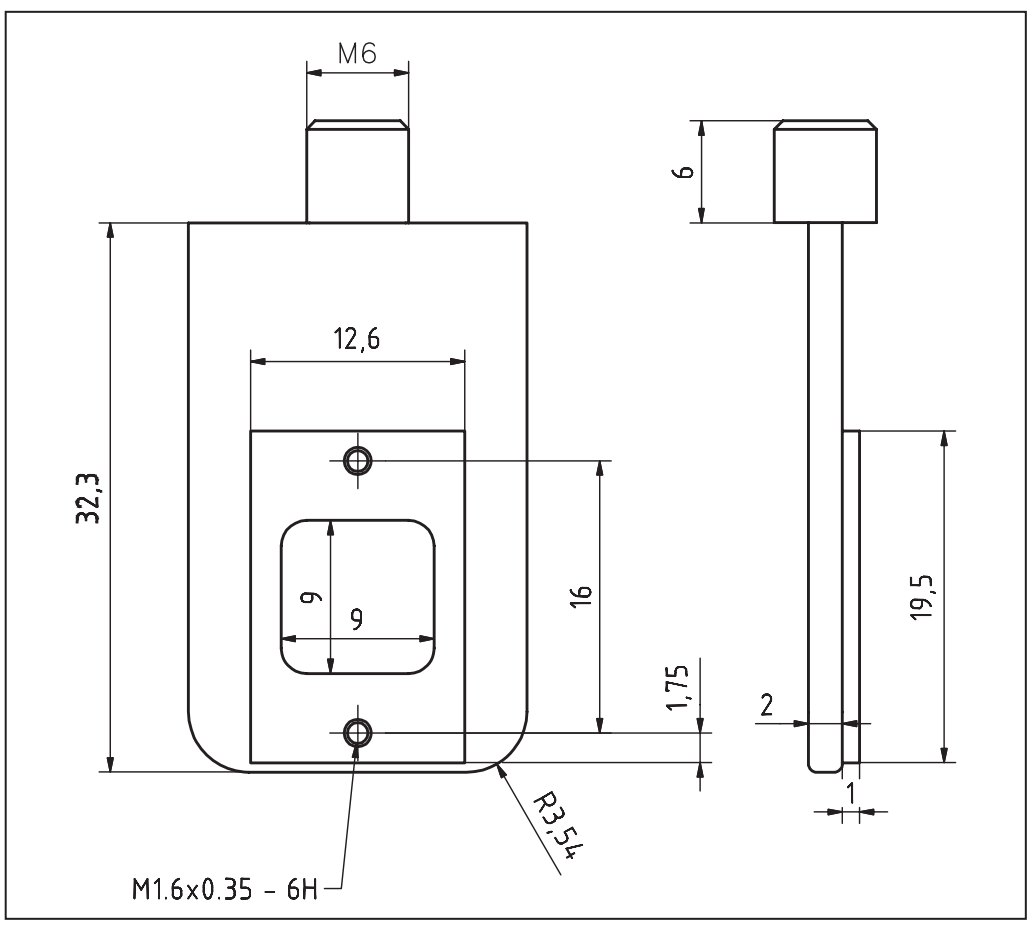

Abbildung 4.4: Konstruktionszeichnung des Substrathalters mit dem Rahmen zur Probenhalterung (alle Maße in $[\mathrm{mm}]$ ). Zeichnung: Feinmechanikwerkstatt des II. Physikalischen Instituts, Uni Göttingen.

Temperatur des Heizers nicht über etwa $850^{\circ} \mathrm{C}$ steigen zu lassen, um die mechanische Stabilität des Geräteaufbaus nicht zu gefährden. Durch Wärmeübergangsverluste vom Heizer zur Kupferprobenaufnahme ergibt sich dadurch eine maximale Substrattemperatur von etwa $800{ }^{\circ} \mathrm{C}$.

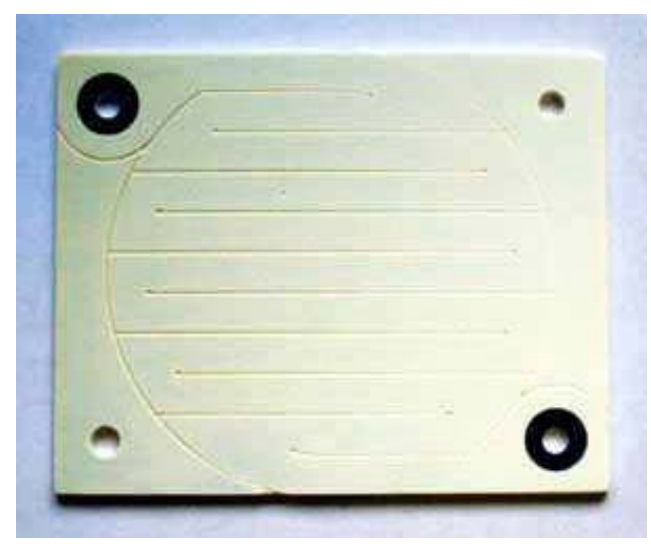

Abbildung 4.5: Boralectric ${ }^{\circledR}$-Heizelement, das zur Substratheizung während der Depositionen verwendet wird. Die dunkel eingefassten Bohrungen sind die Graphitkontakte zur elektrischen Kontaktierung. Durch die beiden anderen Bohrungen wird das Heizelement an der Probenaufnahme festgeschraubt (Foto: Tectra $\mathrm{GmbH}$ ). 


\subsection{Ionenquelle}

Als Ionenstrahlquelle in dieser PLD-Anlage dient ein Tectra IonEtch Ionenstrahl (Tectra GmbH, Frankfurt/Main). Das Gerät verfügt über eine filamentlose Mikrowellenentladungsquelle mit einem Quellenstrom von maximal $1 \mathrm{~mA}$. Die Stromdichte liegt bei einer Entfernung von $100 \mathrm{~mm}$ bei maximal $120 \mu \mathrm{A} / \mathrm{cm}^{2}$. Die Beschleunigungsspannung lässt sich von 25 bis $5000 \mathrm{~V}$ frei regeln. Der Ionenstrahl hat - abhängig von der Ionensorte eine Divergenz von etwa $15^{\circ}$.

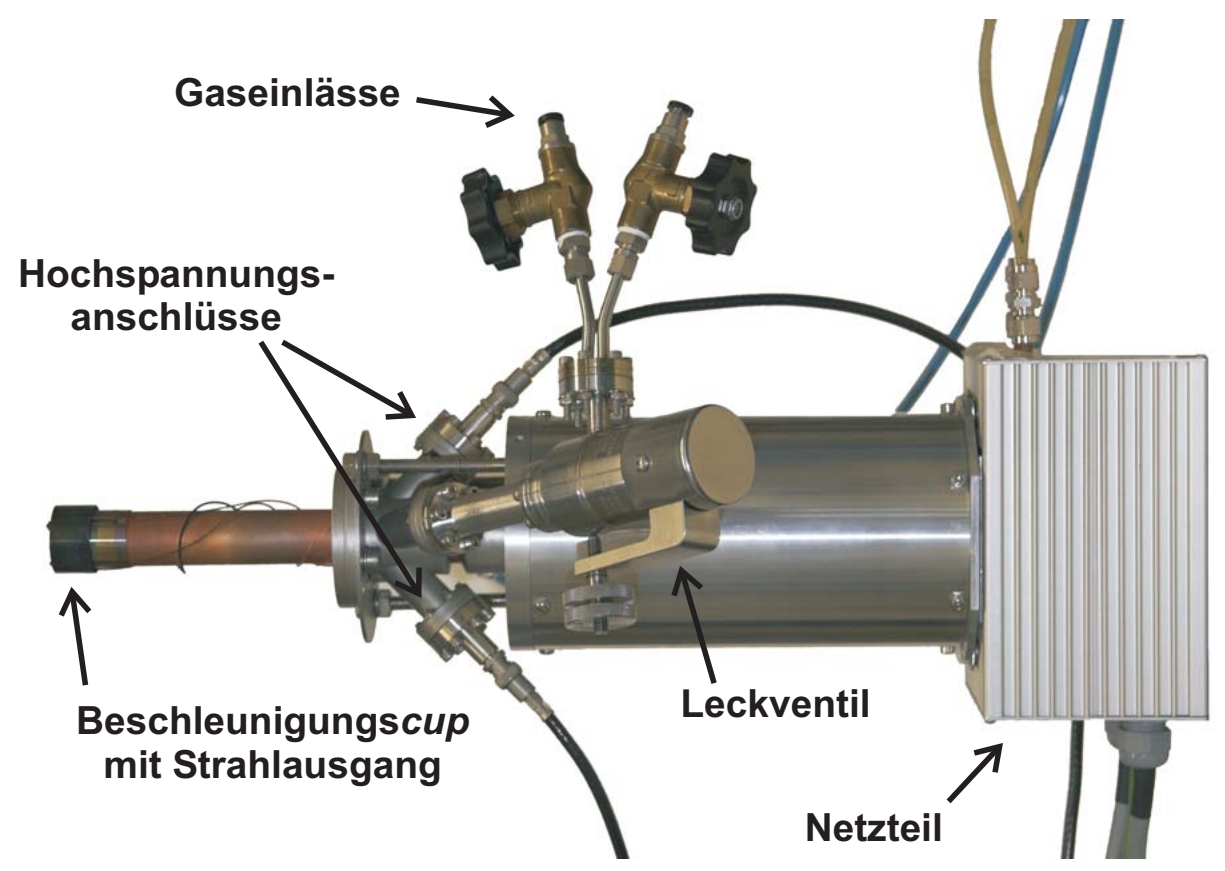

Abbildung 4.6: Abbildung der in der hier beschriebenen Kammer eingebauten IonEtchIonenquelle.

In obiger Abbildung (Abb. 4.6) ist die in der Kammer eingebaute Ionenquelle dargestellt. Am Einlass-Leckventil des Gerätes wurde ein Flansch mit zwei unabhängigen Gaseinlässen angebaut. So ist es möglich verschiedene Gase (z.B. $\mathrm{N}_{2}$ und Ar) gleichzeitig verfügbar zu halten, ohne aufwändig die angeschlossenen Gasleitungen wechseln zu müssen. Die Gase liegen von außen mit einem Druck von etwa 1,5-2 bar an. Zur Benutzung des Ionenstrahls wird in der Regel mit dem Leckventil ein Druck von $5 \cdot 10^{-4}$ mbar eingestellt und anschließend das Plasma in der Mikrowellenquelle gezündet. (Geringere Gasdrücke sind grundsätzlich möglich. Allerdings ist dabei zu beachten, dass in Abhängigkeit von der Ionisierungsfähigkeit des verwendeten Gases das Plasma dann zunehmend instabil wird.) Danach sollte über einen Zeitraum von etwa 15 Minuten der Magnetronstrom von 15 auf maximal $25 \mathrm{~mA}$ langsam erhöht werden, um das Plasma zu stabilisieren. Anschließend kann die gewünschte Beschleunigungsspannung angelegt werden. Genauere Betriebsanweisungen finden sich in der technischen Dokumentation zum Gerät. Das Foto in Abbildung 
4.7 zeigt die in die Kammer eingebaute Ionenquelle, sowie den Probenhalter, auf den die Ionenquelle im $45^{\circ}$-Winkel ausgerichtet ist.

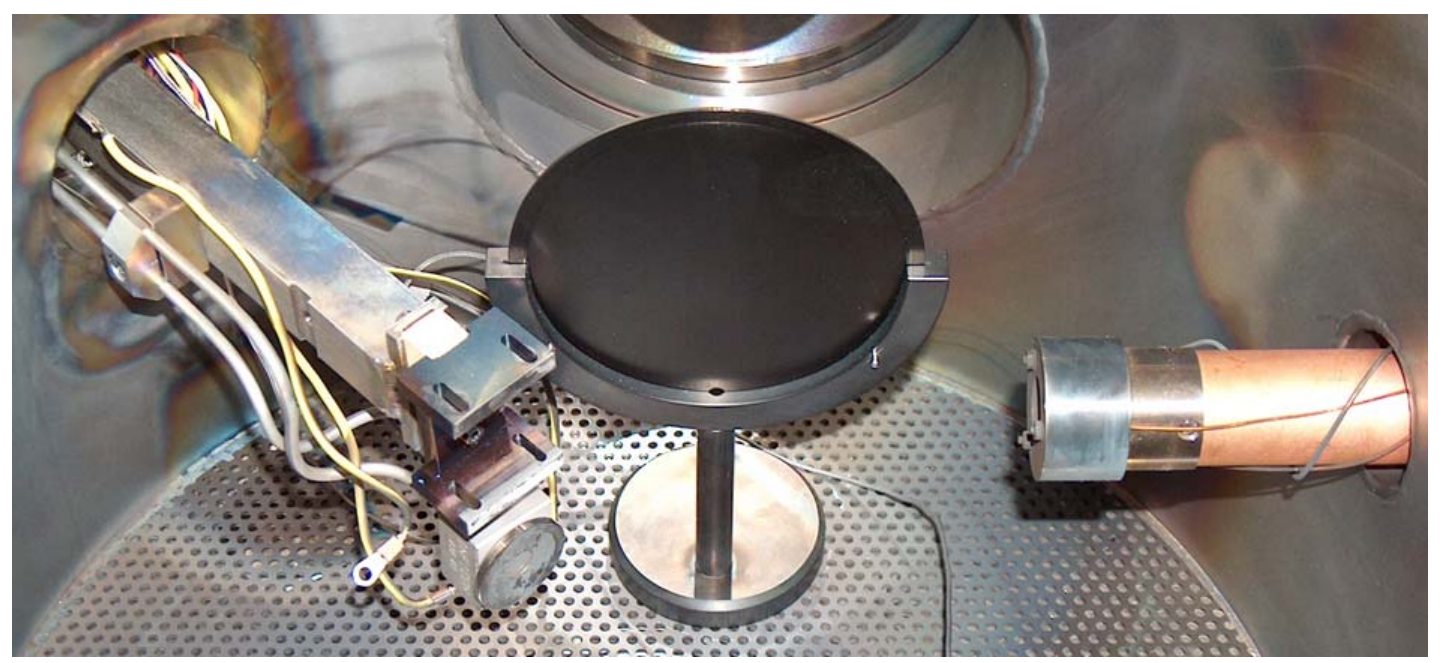

Abbildung 4.7: Abbildung der Kammer mit eingebauter Ionenquelle, die im 45-Winkel auf den Probenhalter ausgerichtet ist.

\subsection{Vakuumpumpen}

Als Vakuumpumpensystem dient die Kombination aus einer luftgekühlten Drehschieberpumpe TRIVAC D4B $\left(4 \mathrm{~m}^{3} / \mathrm{h}\right)$ und einer wassergekühlten Turbomolekularpumpe TURBOVAC 151 (145 l N 2/s), gesteuert durch das Steuergerät TURBOTRONIK NT 151/361 (alles Leybold Vakuum GmbH, Köln). Mit diesen Pumpen lässt sich ein Hochvakuum von $10^{-5} \mathrm{~Pa}\left(=10^{-7} \mathrm{mbar}\right)$ in der Kammer einstellen. Die Druckmessung erfolgt für ein Vakuum bis $1 \cdot 10^{-4}$ mbar durch zwei Pirani-Messzellen THERMOVAC TR211 (Leybold-Heraeus GmbH, Köln). Zur Hochvakuummessung steht ein Kaltkathoden (Penning)-Vakuumsensor PENNINGVAC PR35 (Leybold-Heraeus GmbH, Köln) zur Verfügung. Alle Sensoren sind an ein Steuergerät COMBIVAC CM31 (Leybold-Heraeus GmbH, Köln) angeschlossen. Abbildung 4.8 zeigt den Schaltplan mit allen Pumpen und Ventilen der Anlage.

Beim Evakuieren der Kammer ist darauf zu achten, dass der maximale Vordruck der Turbomolekularpumpe (TMP) bei $5 \cdot 10^{-1}$ mbar liegt. Vor Inbetriebnahme der TMP und Öffnen des Schiebeventils V3 zwischen Vakuumkammer und TMP ist deswegen die gesamte Anlage mit der Vorpumpe über das Schiebeventil V2 auf einen Druck kleiner $10^{-1}$ mbar abzupumpen (Kontrolle über die Pirani-Messzellen). Dabei sollte bei still stehender TMP bereits Ventil V5 geöffnet sein, um den Raum hinter der TMP zu evakuieren. Ist die Kammer entsprechend vorevakuiert, wird die TMP gestartet und nach entsprechender Anlaufzeit durch Schließen von V2 und Öffnen von V3 in das System geschaltet. Das 


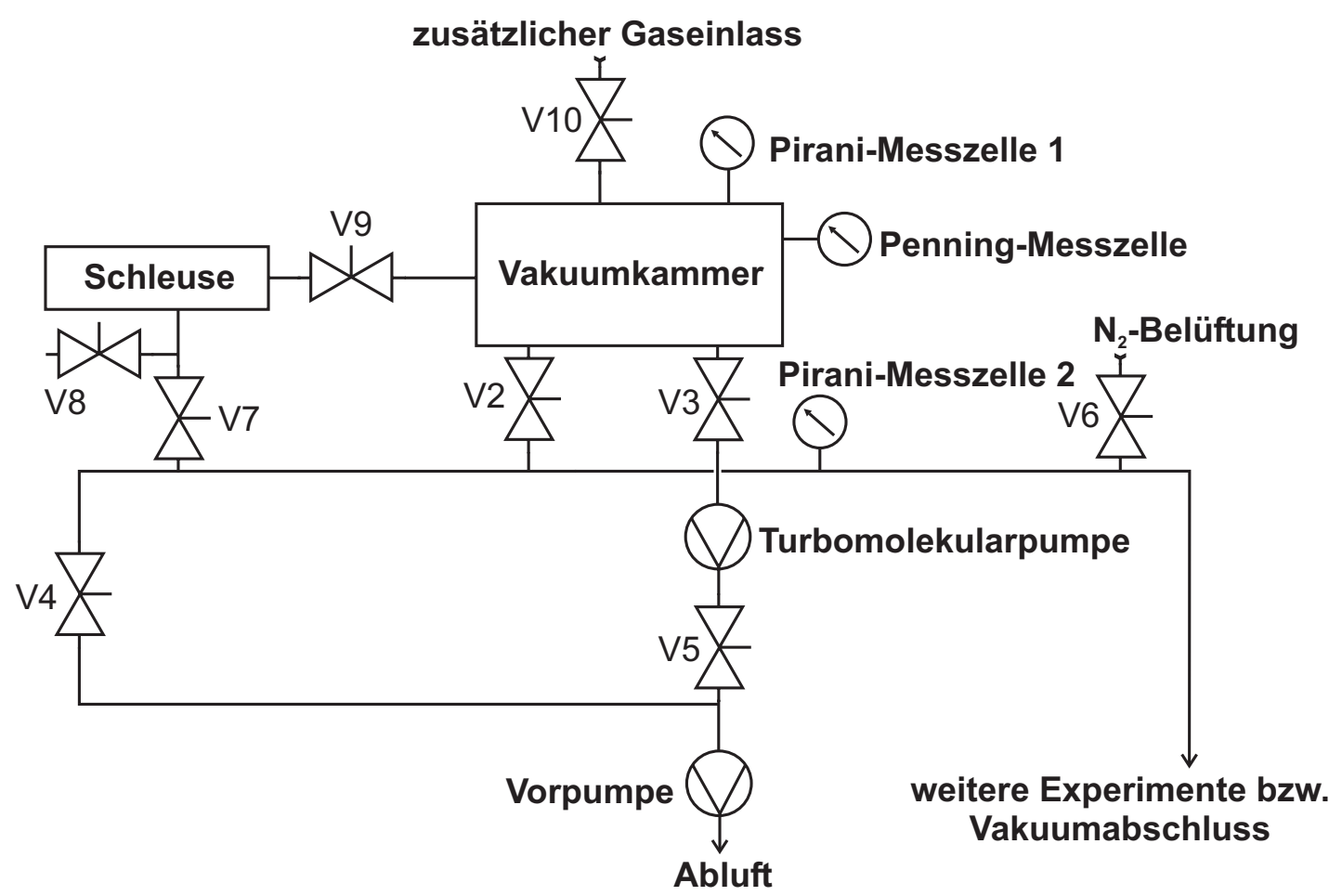

Abbildung 4.8: Schaltplan der Pumpen, Ventile und Mess-Sensoren der PLD-Anlage.

Vakuum von $10^{-7}$ mbar kann dabei nur erreicht werden, wenn das Probentransferventil V9 geschlossen ist. Während der Deposition muss also der Substrathalter von der Transferstange geschraubt und die Stange aus der Kammer zurückgezogen werden, um V9 zu schließen.

Zum Probenwechsel wird die Schleuse bei geschlossenen Ventilen V7 und V9 über das Ventil V8 belüftet. Nach Einbau der Probe wird die Probenschleuse mit Hilfe der Vorpumpe über V7 abgepumpt. Wichtig ist dabei, VOR Öffnen von V7 das Ventil V5 zu schließen, da sonst Umgebungsdruck aus der Schleuse an die TMP gelangt. Ist die Schleuse auf etwa $10^{-2}$ mbar evakuiert, wird V7 geschlossen, V5 wiederum geöffnet und die Probe kann über V9 in die Kammer eingeschleust werden. Weitere Ventile sind das Nadelventil V10 zum Einlass von Hintergrundgasen und das Belüftugsventil V6 zum Fluten der Kammer mit Stickstoff. Bei der Benutzung von Hintergrundgasen ist darauf zu achten, dass der maximale Belastungsdruck der TMP $5 \cdot 10^{-2}$ mbar beträgt. Höhere Hintergrunddrücke führen bei zugeschalteter TMP zur Beschädigung der Pumpe. Das Ventil V4 ist während des PLD-Betriebs ständig offen. Es dient lediglich dazu, beim Anschluss einer kleineren Laserbestrahlungskammer diese über die Vorpumpe zu evakuieren. 


\subsection{Targethalter}

Als Targethalter dient ein drehbar gelagertes Aluminiumsechseck (Seitenlänge 62,5 mm, Höhe $30 \mathrm{~mm}$ ) auf dessen Flächen jeweils ein Target befestigt werden kann. Abbildung 4.9 zeigt die Konstruktionszeichnung dieses Bauteils. Über eine an das Sechseck angeschraubte Welle ist der Halter mit einer außerhalb der Kammer am Kammerdeckel fixierten Aufnahme verbunden. Die Welle ermöglicht gleichzeitig die volle Rotation um $360^{\circ}$ in beide Richtungen. Diese Aufnahme - und damit auch der Targethalter - sind so im Kammerdeckel positioniert, dass bei paralleler Ausrichtung der Sechseckflächen zum Substrat der Laserstrahl genau auf die Flächenmitte in einem Winkel von $45^{\circ}$ auftrifft. Der Abstand zwischen Target und Substrat beträgt etwa $70 \mathrm{~mm}$.

In Abschnitt 2.2 wurde bereits gezeigt, dass es bei der PLD zur Herstellung qualitativ guter Schichten notwendig ist, das Target während der Ablation zu bewegen. Einerseits dient dies dazu, eine homogenere Materialabtragung auf dem Target - und damit eine geringere Zahl von droplets - zu gewährleisten, und andererseits wird durch die Bewegung der Plasmafackel relativ zum Substrat eine homogenere Schichtabscheidung ermöglicht. Aus diesem Grund ist es möglich, den Targethalter dieser Kammer mit zwei verschiedenen Motoren in zwei Richtungen zu bewegen.

Eine Bewegung ist die entlang der z-Achse, d.h. eine auf-und-ab-Bewegung relativ zum feststehenden Laserstrahl. Dazu dient ein 24V-Gleichstrommotor (Crouzet Automatismes SAS, Valence, Frankreich), der die gesamte Halteraufnahme über einen Schneckentrieb nach oben und unten bewegen kann. Die Endpunkte dieser Bewegung werden durch eine Eigenbau-Motorsteuerung der Elektronikwerkstatt des II. Physikalischen Instituts der Universität Göttingen kontrolliert. Dabei können oberer und unterer Umkehrpunkt in einem Bereich von $\pm 15 \mathrm{~mm}$ von der mittleren Motorposition mit einer Genauigkeit von 0,1 mm festgelegt werden. Die Positionskontrolle erfolgt über die Messung des elektrischen Widerstands eines an die Schneckenwelle angeschlossenen Potentiometers. Diese Mechanik erlaubt damit nicht nur die Vergrößerung der vom Laser ablatierten Fläche, sondern auch die Abrasterung eines Multielementtargets in gewünschten Mengenverhältnissen entlang der z-Achse. Diese Möglichkeit zur Verhältniskontrolle bei der Ablation von mehreren Elementen auf einem Target wurde im Rahmen dieser Arbeit bei der Deposition von Titan und Aluminium (siehe Kapitel 5) und des Weiteren auch in [134] und [135] genutzt.

Die zweite mögliche Bewegung ist die Rotationsbewegung um den Sechseckmittelpunkt mit z-Achse als Rotationsachse. Dies geschieht durch die kontrollierte Bewegung der am Targethalter angeschraubten Welle. Zur Bewegung dient ein computergesteuerter Mikroschrittmotor PANdrive PD-109-57 (TRINAMIC Motion Control GmbH \& Co. KG, Hamburg). Dieser Schrittmotor lässt sich über eine serielle RS232-Schnittstelle und der vom Hersteller entwickelten Programmiersprache Trinamic Motion Control Language (TMCL) 


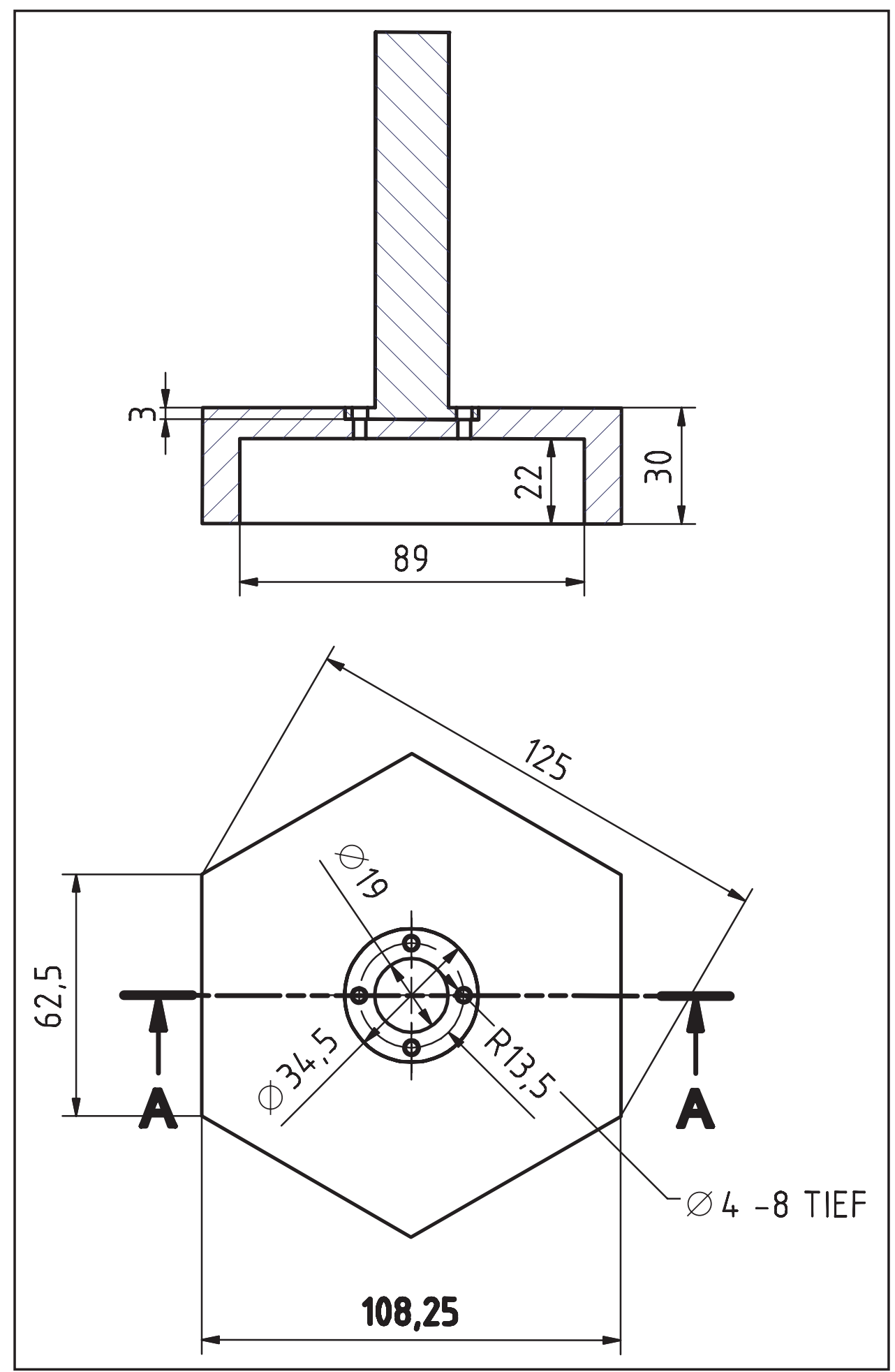

Abbildung 4.9: Konstruktionszeichnung des Targethalters (Zeichnung: Feinmechanikwerkstatt des II. Physikalischen Instituts, Uni Göttingen).

steuern. In den hier beschriebenen Arbeiten wurde damit das Target während der Ablation jeweils $3^{\circ}$ in beide Richtungen aus der parallelen Stellung zwischen Targetoberfläche und Substrat ausgelenkt. Auf diese Weise ist es möglich, den vom Laser bestrahlten Be- 
reich auf mehr als die dreifache Fläche zu vergrößern und eine homogene Schichtdicke auf der gesamten Substratfläche zu erreichen. An der Kammer eingebaut ist bereits eine Kombination aus Photodiode und Photosensor, die durch Reflektion die korrekte Parallelausrichtung des Targethalters erfassen kann. Dadurch kann dem Schrittmotor eine Referenzposition zugewiesen werden, von der aus jede der sechs Targetflächen schnell und reproduzierbar in den Laserstrahl gebracht werden kann. Durch eine entsprechende Synchronisation mit dem ebenfalls über RS232 steuerbaren Laser (s. Abschnitt 4.6) können dann verschiedene Ablationstargets während der Deposition bestrahlt werden, ohne das Experiment unterbrechen zu müssen. Aus Zeitgründen wurde diese Funktion aber softwareseitig noch nicht implementiert. Der komplette Aufbau der PLD-Kammer mit den oben beschriebenen Elementen zur Bewegung des Targethalters ist in Abbildung 4.10 gezeigt.

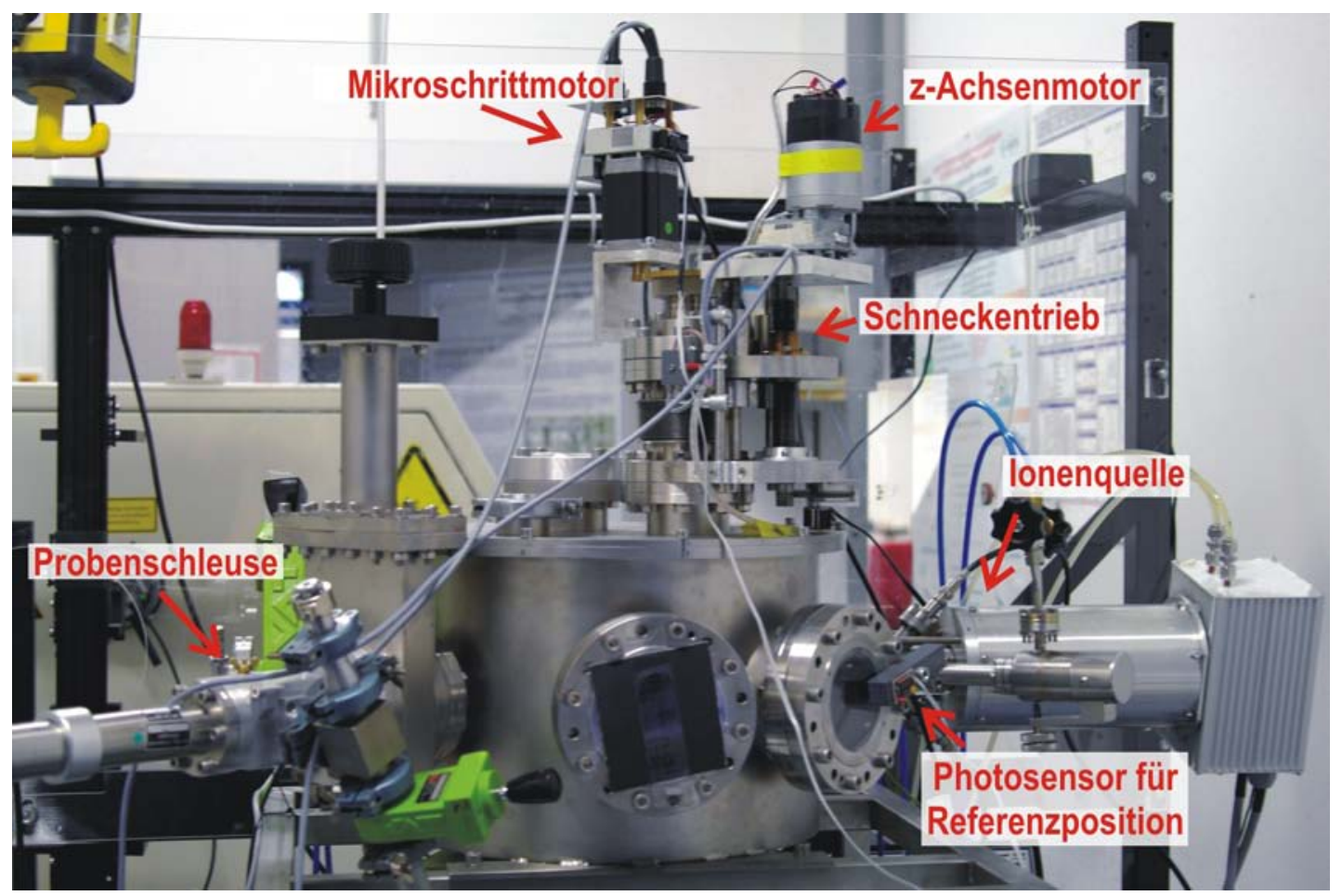

Abbildung 4.10: Aufnahme der PLD-Anlage mit den Elementen zur Targetbewegung. Der Laser befindet sich vom Betrachter aus hinter der Vakuumkammer.

\subsection{Laser}

Grundsätzlich erlaubt der Aufbau der Kammer die Benutzung jedes beliebigen (PLDfähigen) Lasers. Die hier beschriebenen Depositionen wurden mit einem gepulsten Nd:YAGLaser mit Güteschaltung Quantel Brilliant (Quantel S.A., Les Ulis, Frankreich) mit einer Pulsdauer von $\tau=6 \mathrm{~ns}$ und einer Pulsfrequenz von $f=20 \mathrm{~Hz}$ durchgeführt. Der Laser hat bei Betrieb mit der ersten Harmonischen $(\lambda=1064 \mathrm{~nm})$ eine Ausgangsleistung 
von $360 \mathrm{~mJ} /$ Puls. Durch Anbau von Frequenzvervielfachern ist es möglich, den Laser auch mit der zweiten $(\lambda=532 \mathrm{~nm})$ und dritten Harmonischen $(\lambda=355 \mathrm{~nm})$ zu betreiben. Die Ausgangsleistungen verringern sich dann auf $160 \mathrm{~mJ} / \mathrm{Puls}$ (532 nm) bzw. $60 \mathrm{~mJ} /$ Puls (355 nm). Der Rohstrahl des Lasers hat einem Durchmesser von $6 \mathrm{~mm}$. Das ergibt beim Betrieb mit $1064 \mathrm{~nm}$ eine Laserfluenz von 1,24 J/ $\mathrm{cm}^{2}$. Der Laser ist auf einem höhenverstellbaren Gestell installiert, an dem sich die Linse zur Strahlfokussierung frei entlang der optischen Achse verschieben lässt. Auf diese Weise ist es möglich, die Fleckgröße des Laserstrahls - und damit die Laserfluenz - stufenlos einzustellen. Die Linsen sind antireflexbeschichtete (Reflektivität bei der gewählten Wellenlänge $<0,5 \%$ ) Plankonvexlinsen (LINOS AG, Göttingen). Aufgrund der wellenlängenabhängigen Beschichtung ist es notwendig, für jede der drei Betriebswellenlängen des Lasers eine angepasste Linse zu verwenden. Die Linsen sind deswegen einzeln in Halterungen gefasst und können entsprechend an der beweglichen Halterung gewechselt werden. Folgende Linsen stehen an der Anlage zur Verfügung:

- N-BK7 ${ }^{\circledR}$ Schott AG, Mainz ARHS-1064-Beschichtung, f = $500 \mathrm{~mm}$ für $\lambda=1064 \mathrm{~nm}$,

- N-BK7 ${ }^{\circledR}$ ARB2-VIS-Beschichtung, $\mathrm{f}=500 \mathrm{~mm}$ für $\lambda=532 \mathrm{~nm}$ und

- Quarzglas ARB2-UV-Beschichtung, $\mathrm{f}=500 \mathrm{~mm}$ für $\lambda=355 \mathrm{~nm}$

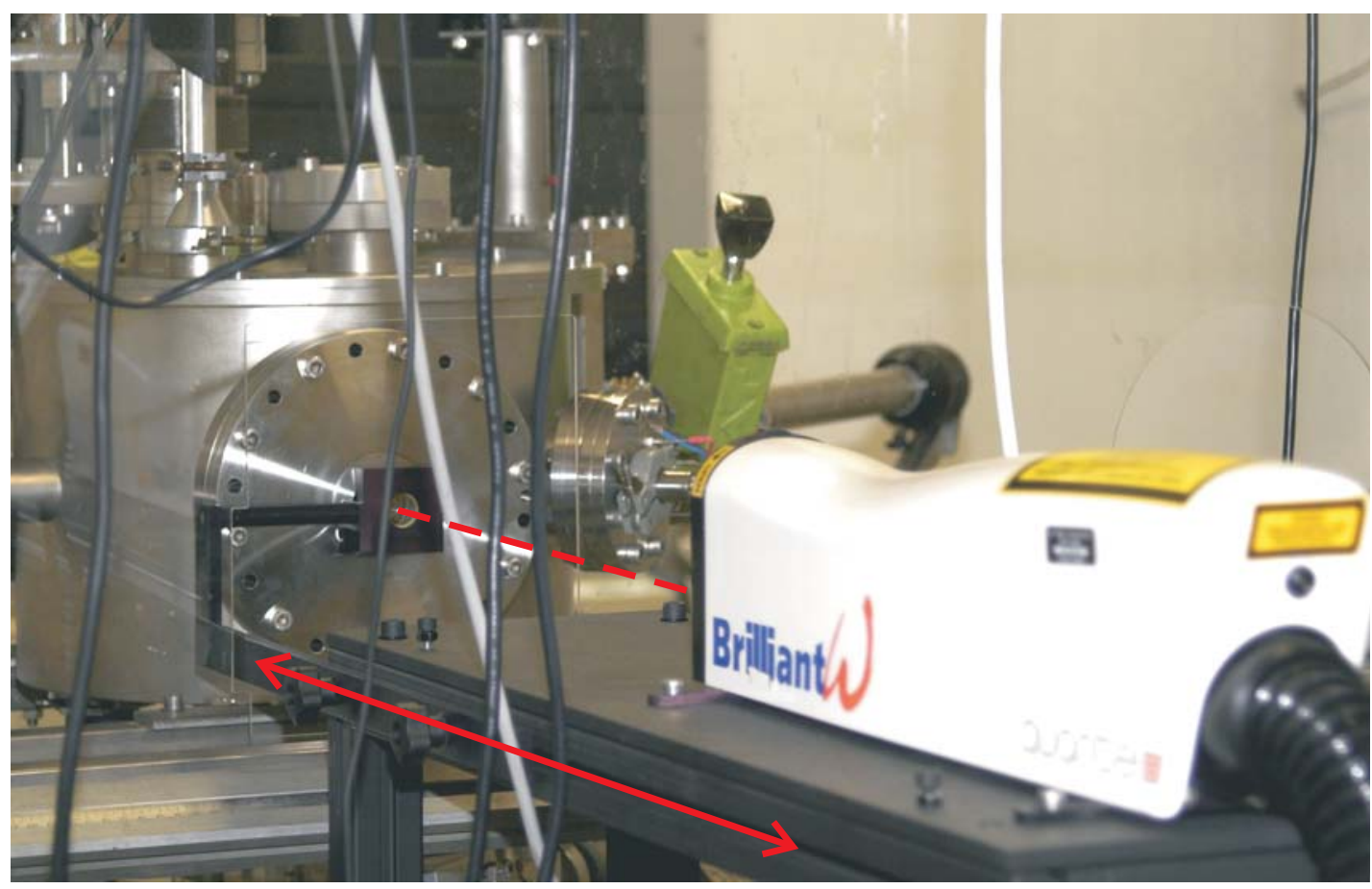

Abbildung 4.11: Aufbau des Lasers und der Fokussierlinse an der PLD-Anlage. Eingezeichnet sind der Strahlengang des Lasers (Strichlinie) und die verschiebbare Linsenhalterung (Pfeile). 
Den Aufbau des Lasers und der Fokussieroptik an der Kammer zeigt das Foto in Abbildung 4.11. Der Laser lässt sich über eine Fernbedienung in allen Funktionen per Hand steuern. Eine Computersteuerung über die serielle Schnittstelle RS232 mit einer Synchronisation des Targethalters (s. Abschnitt 4.5) ist in der Entwicklung, jedoch noch nicht fertiggestellt. 


\section{Herstellung der Proben}

Alle Proben im Rahmen dieser Arbeit wurden mit der in Kapitel 4 beschriebenen PLDAnlage hergestellt. Da es sich hier um erste Untersuchungen zur PLD von MAX-Phasen an diesem Experiment handelt, war das Ziel der Arbeit, zunächst einen großen Parameterraum bei den Depositionen zu bearbeiten. Dabei wurden Untersuchungen in drei verschiedenen MAX-Systemen durchgeführt, nämlich Ti-Si-C, Cr-Al-C und Ti-Al-N.

Abbildung 5.1 illustriert den Aufbau des Experiments zur Synthese der dünnen Schichten. Der Strahl des Pulslasers ist fest positioniert und fällt in einem Winkel von $45^{\circ}$ auf die Targetoberfläche, die parallel zur Substratoberfläche ausgerichtet ist. Der Targethalter bewegt sich während der Ablation in beide Richtungen um $3^{\circ}$ aus dieser $45^{\circ}$-Position hinaus. Dadurch werden (a) das Target homogener ablatiert und (b) durch die Bewegung der Plasmafackel eine homogenere Beschichtung des Substrats erreicht. Bei einem Teil der Experimente wurde zusätzlich ein Ionenstrahl (inert: Ar und reaktiv: $\mathrm{N}_{2}$ ) im Winkel von $45^{\circ}$ auf das Substrat gerichtet.

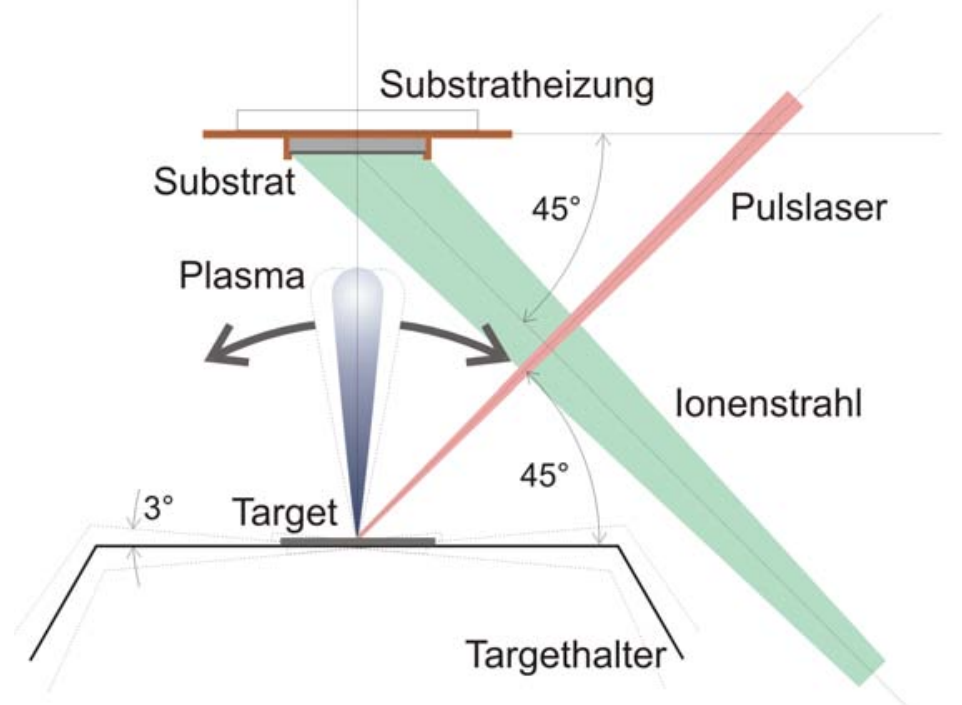

Abbildung 5.1: Zeichnung des zur Schichtdeposition verwendeten Experiments mit Laserstrahlengang und Ionenstrahl.

Für die Systeme Ti-Si-C und Cr-Al-C wurden vorsynthetisierte MAX-Phasen-Ablationstargets der Zusammensetzungen $\mathrm{Ti}_{3} \mathrm{SiC}_{2}$ bzw. $\mathrm{Cr}_{2} \mathrm{AlC}$ verwendet. Diese Targets wur- 
den in der Arbeitsgruppe Barsoum an der Drexel University (Philadelphia, USA) hergestellt. Das $\mathrm{Cr}_{2} \mathrm{AlC}$-Target ist laut $\mathrm{AG}$ Barsoum phasenrein, im $\mathrm{Ti}_{3} \mathrm{SiC}_{2}$-Target liegt zusätzlich ein Anteil von etwa $3 \% \mathrm{TiC}_{x}$ in der $\mathrm{Ti}_{3} \mathrm{SiC}_{2}$-Matrix vor. Die Charakterisierung mit GDOES und XRD der $\mathrm{Ti}_{3} \mathrm{SiC}_{2}$-und $\mathrm{Cr}_{2} \mathrm{AlC}$-Ablationstargets ist in den Abbildungen 5.2 und 5.3 dargestellt. Die GDOES-Aufnahmen zeigen, dass beide MAX-Targets in der gewünschten chemischen Zusammensetzung vorliegen. Das $\mathrm{Cr}_{2} \mathrm{AlC}$-Target ist auch kristallographisch phasenrein, wie die indizierten $\mathrm{Cr}_{2}$ AlC-Reflexe (ICSD-Datenblatt 01089-2275) zeigen. Im $\mathrm{Ti}_{3} \mathrm{SiC}_{2}$-Target findet man ebenfalls vorwiegend die Reflexe der MAX-Phase (ICSD-Datenblatt 40-1132). Die zusätzlichen, nicht indizierten Reflexe lassen sich einerseits $\mathrm{TiC}_{x}$ und andererseits oxidischen Verunreinigungen an der Oberfläche zuordnen.
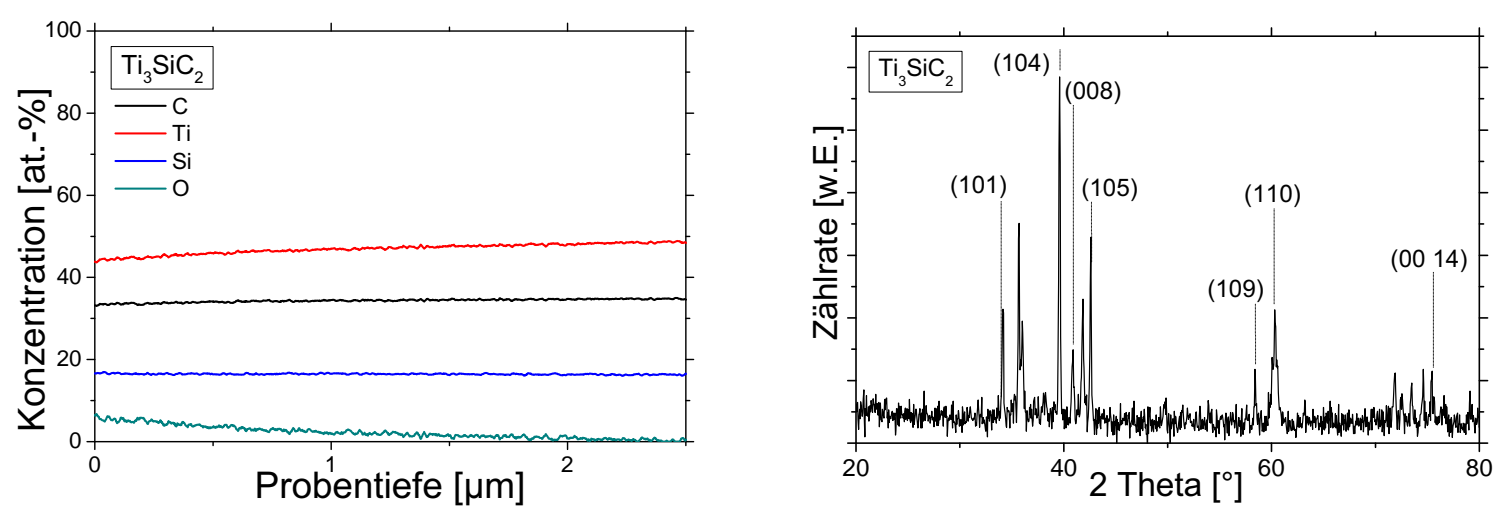

Abbildung 5.2: GDOES-Aufnahme (links) und XRD-Aufnahme (rechts) des $\mathrm{Ti}_{3} \mathrm{SiC}_{2}$-Ablationstargets. Die GDOES-Aufnahme zeigt die gewünschte Zusammensetzung von 50 at.-\% Ti, 33 at.- $\% \mathrm{C}$ und 17 at.- $\% \mathrm{Si}$, sowie einen geringen Anteil von Oxiden an der Oberfläche. Im Röntgendiffraktogramm sind die Millerschen Indizes der $\mathrm{Ti}_{3} \mathrm{SiC}_{2}$-Reflexe angegeben. Nicht indizierte Reflexe stammen von einem geringen Anteil $\mathrm{TiC}_{x}$ (ca. $3 \%$ ) in der Targetmatrix, bzw. den oxidischen Verunreinigungen an der Oberfläche.

Die Synthese der Schichten im System Ti-Al-N erfolgte nach einem anderen Prinzip. Dabei wurden die reinen Elemente Aluminium (99,0 \%) und Titan (99,6\%, beide Goodfellow GmbH, Bad Nauheim) im Verhältnis 1:2 in einer Stickstoffatmosphäre ablatiert.

Zur Synthese der Schichten wurde ein gepulster Nd:YAG-Laser Quantel Brilliant (Quantel S.A., Les Ulis, Frankreich) mit einer Pulsdauer von $\tau=6$ ns und einer Pulsfrequenz von $f=20 \mathrm{~Hz}$ genutzt. Zur Ablation wurden die erste Harmonische $(\lambda=1064 \mathrm{~nm})$ verwendet. Die Laserfluenz betrug immer $4,5 \mathrm{~J} / \mathrm{cm}^{2}$ und die Depositionszeit 30 Minuten. Als Substratmaterialien dienten $\mathrm{Si}(100)$ (Si-Mat, Landsberg am Lech), amorphes $\mathrm{SiO}_{2}$ (oxidiertes $\mathrm{Si}(100)$ ), $\mathrm{MgO}(100)$ (CrysTec GmbH, Berlin) und polierter Edelstahl (Goodfellow GmbH, Bad Nauheim). Vor der Deposition wurden die Si(100)-Substrate mit einem 2 kV 

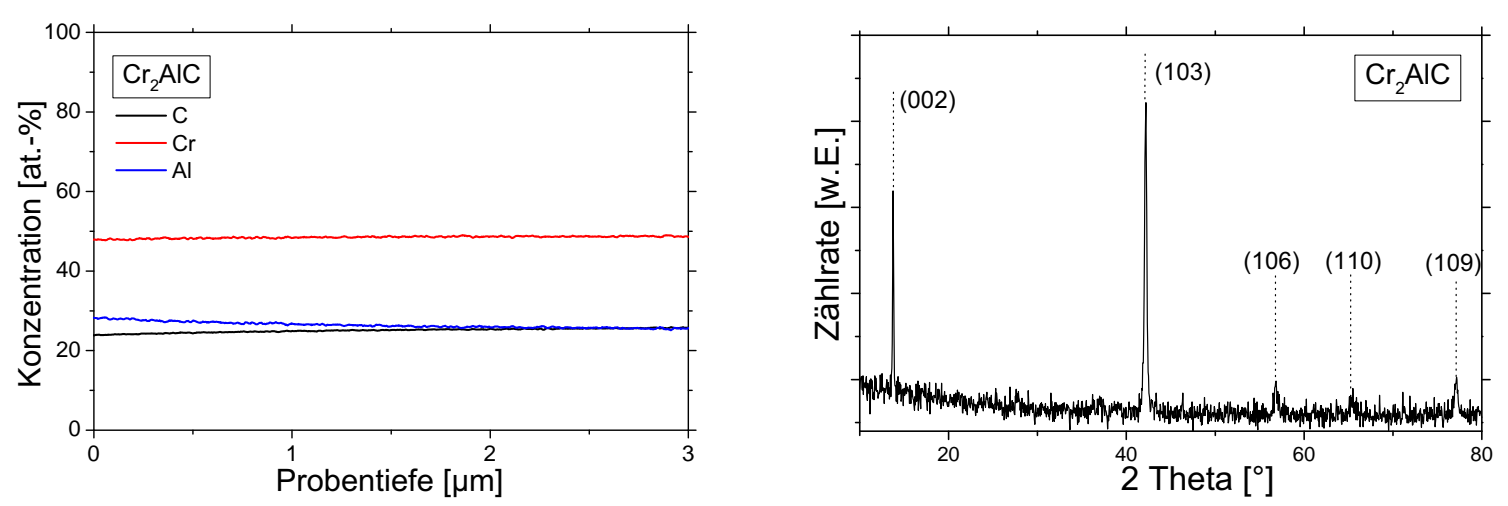

Abbildung 5.3: GDOES-Aufnahme (links) und XRD-Aufnahme (rechts) des $\mathrm{Ti}_{3} \mathrm{SiC}_{2}{ }^{-}$ Ablationstargets. Die GDOES-Aufnahme zeigt die gewünschte Zusammensetzung von 50 at.-\% Cr, 25 at.- \% C und 25 at.-\% Al und eine leichte Kohlenstoffverarmung an der Oberfläche. Im Röntgendiffraktogramm sind die Millerschen Indizes der $\mathrm{Cr}_{2} \mathrm{AlC}-$ Reflexe angegeben.

Ar-Ionenstrahl für 15 Minuten im Vakuum gesputtert, um die anhaftende dünne Oxidschicht zu entfernen. Zur Herstellung der $\mathrm{SiO}_{2}$-Substrate wurden die Siliziumwafer $24 \mathrm{~h}$ bei $1100{ }^{\circ} \mathrm{C}$ in Umgebungsatmosphäre oxidiert. Für die Präparation der Edelsubstrate wurden $1 \mathrm{~mm}$ dicke und 10x10 $\mathrm{mm}^{2}$ große Edelstahlblättchen (AISI 314) zunächst mit SiC-Schleifpapier (Körnung 500, 1200, 2400, 4000) geschliffen und anschließend mit Diamantsuspension $(6 \mu \mathrm{m}$ und $3 \mu \mathrm{m})$ poliert. Die Endrauigkeit der Substrate betrug $R_{a} \approx 15 \mathrm{~nm}$.

Die Depositionen wurden entweder im Hochvakuum $\left(10^{-5} \mathrm{~Pa}\right)$ oder mit Hintergrundgasen (auch bei Anwendung des Ionenstrahls) bei einem Druck von 5·10-2 Pa durchgeführt. Folgende Gase wurden dabei verwendet: Argon (99,998 \%, Air Liquide GmbH, Kassel) und Stickstoff (99,999 \%, Messer Griesheim GmbH Krefeld). Da die verwendeten Targets aufgrund der Lagerung im Labor oberflächlich oxidiert waren, wurden sie nach dem Einbau ins Vakuum und vor den Depositionen zunächst 15 Minuten unter Versuchsbedingungen ablatiert, um die Oberflächen von den Verunreinigungen zu befreien.

Die Tabellen 5.1, 5.2 und 5.3 enthalten Übersichten aller hergestellten Proben in den Systemen Ti-Si-C, Cr-Al-C bzw. Ti-Al-N mit den entsprechenden experimentellen Parametern. Im den Systemen Ti-Si-C und Cr-Al-C wurden Schichten bei verschiedenen Substrattemperaturen auf verschiedenen Substratmaterialien abgeschieden. Diese Versuchsreihen wurde dann mit zugeschaltetem Ar-Ionenstrahl wiederholt, um Aufschlüsse über einen möglichen Einfluss des Ionenbeschusses zu erlangen. Bei der reaktiven PLD im System Ti-Al-N wurde nur ein Substratmaterial $\mathrm{MgO}(100)$ verwendet. Bei Substrattemperaturen von $450{ }^{\circ} \mathrm{C}$ und $650{ }^{\circ} \mathrm{C}$ wurde dabei immer der Stickstoff-Ionenstrahl mit Beschleunigungsspannungen von 0 bis $1000 \mathrm{~V}$ auf das Substrat gerichtet. 
Tabelle 5.1: Übersicht aller in dieser Arbeit betrachteten Ti-Si-C-Proben mit den entsprechenden experimentellen Parametern. T bezeichnet die Substrattemperatur während der Deposition.

\begin{tabular}{|c|c|c|}
\hline Substrat & Feste Parameter & Veränderliche Parameter \\
\hline $\mathrm{MgO}(100)$ & $\cdot \mathrm{UHV}, \lambda=1064 \mathrm{~nm}$ & $\mathrm{~T}=\mathrm{RT}-650^{\circ} \mathrm{C}$ \\
& $200 \mathrm{~V}$ Ar-Ionenstrahl, & $\mathrm{T}=\mathrm{RT}-650^{\circ} \mathrm{C}$ \\
& $\lambda=1064 \mathrm{~nm}$ & \\
\hline $\mathrm{Si}(100)$ & $\cdot \mathrm{UHV}, \lambda=1064 \mathrm{~nm}$ & $\mathrm{~T}=\mathrm{RT}-650{ }^{\circ} \mathrm{C}$ \\
& $\cdot 200 \mathrm{~V}$ Ar-Ionenstrahl, & $\mathrm{T}=\mathrm{RT}-650{ }^{\circ} \mathrm{C}$ \\
& $\lambda=1064 \mathrm{~nm}$ & \\
\hline Edelstahl & $\cdot \mathrm{UHV}, \lambda=1064 \mathrm{~nm}$ & $\mathrm{~T}=\mathrm{RT}-650^{\circ} \mathrm{C}$ \\
& $\cdot 200 \mathrm{~V}$ Ar-Ionenstrahl, & $\mathrm{T}=\mathrm{RT}-650^{\circ} \mathrm{C}$ \\
& $\lambda=1064 \mathrm{~nm}$ & \\
\hline
\end{tabular}

Tabelle 5.2: Übersicht aller in dieser Arbeit betrachteten Cr-Al-C-Proben mit den entsprechenden experimentellen Parametern. T bezeichnet die Substrattemperatur während der Deposition.

\begin{tabular}{|c|c|c|}
\hline Substrat & Feste Parameter & Veränderliche Parameter \\
\hline $\mathrm{MgO}(100)$ & $\cdot \mathrm{UHV}, \lambda=1064 \mathrm{~nm}$ & $\mathrm{~T}=\mathrm{RT}-650^{\circ} \mathrm{C}$ \\
& $\cdot 200 \mathrm{~V}$ Ar-Ionenstrahl, & $\mathrm{T}=\mathrm{RT}-650^{\circ} \mathrm{C}$ \\
& $\lambda=1064 \mathrm{~nm}$ & \\
\hline $\mathrm{SiO}_{2}$ & $\cdot \mathrm{UHV}, \lambda=1064 \mathrm{~nm}$ & $\mathrm{~T}=\mathrm{RT}-650^{\circ} \mathrm{C}$ \\
& $\cdot 200 \mathrm{~V}$ Ar-Ionenstrahl, & $\mathrm{T}=\mathrm{RT}-650^{\circ} \mathrm{C}$ \\
& $\lambda=1064 \mathrm{~nm}$ & \\
\hline Edelstahl & $\cdot \mathrm{UHV}, \lambda=1064 \mathrm{~nm}$ & $\mathrm{~T}=\mathrm{RT}-650^{\circ} \mathrm{C}$ \\
& $\cdot 200 \mathrm{~V}$ Ar-Ionenstrahl, & $\mathrm{T}=\mathrm{RT}-650^{\circ} \mathrm{C}$ \\
& $\lambda=1064 \mathrm{~nm}$ & \\
\hline
\end{tabular}

Tabelle 5.3: Übersicht aller in dieser Arbeit betrachteten Ti-Al-N-Proben mit den entsprechenden experimentellen Parametern. T bezeichnet die Substrattemperatur während der Deposition, U die Beschleunigungsspannung des Ionenstrahls.

\begin{tabular}{|c|c|c|}
\hline Substrat & Feste Parameter & Veränderliche Parameter \\
\hline $\mathrm{MgO}(100)$ & $\cdot \mathrm{N}_{2}$-Ionenstrahl, & $\mathrm{T}=450{ }^{\circ} \mathrm{C}, 650{ }^{\circ} \mathrm{C}$, \\
& $\lambda=1064 \mathrm{~nm}$ & $\mathrm{U}=0-1000 \mathrm{~V}$ \\
\hline
\end{tabular}




\section{Ergebnisse der Deposition von MAX-Phasen-Targets}

\section{1 $\mathrm{Ti}_{3} \mathrm{SiC}_{2}$-Target}

Abbildung 6.1 zeigt die REM-Aufnahme des Querschnittes einer Schicht, die bei Raumtemperatur vom $\mathrm{Ti}_{3} \mathrm{SiC}_{2}$-Target auf $\mathrm{MgO}(100)$ deponiert wurde. $\mathrm{Zu}$ erkennen ist ein etwa $750 \mathrm{~nm}$ dicker homogener Film. Bis auf eine Ausnahme $\left(\mathrm{Si}(100)\right.$-Substrat, $\mathrm{T}=650{ }^{\circ} \mathrm{C}$, 200 V Ar-Ionenstrahl) gelang in allen Fällen die Deposition solch homogener Filme. Bei der erwähnten Ausnahme kam es jedoch zu einem Abblättern der Schicht vom Substrat noch während der Deposition.

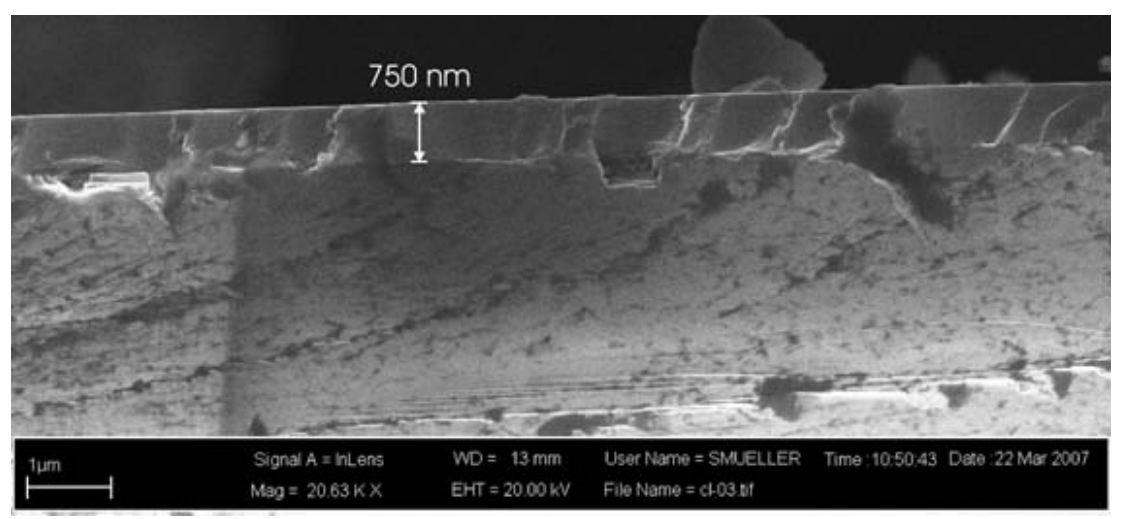

Abbildung 6.1: REM-Aufnahme des Querschnitts durch eine bei Raumtemperatur auf $\mathrm{MgO}(100)$ deponierte $\mathrm{Ti}_{3} \mathrm{SiC}_{2}$-Schicht.

\subsection{1 $\mathrm{MgO}(100)-$ Substrat}

\subsubsection{GDOES-Messungen}

Allen Proben gemeinsam ist, dass sich die chemische Zusammensetzung des $\mathrm{Ti}_{3} \mathrm{SiC}_{2}{ }^{-}$ Targets (50 at.-\% Ti, 17 at.-\% Si und 33 at.-\% C) nicht in den Schichten wieder findet. Statt dessen enthalten die Filme vor allem weniger Silizium als das Target und zeigen 
außerdem einen zum Teil deutlichen Anteil an Sauerstoff. Die mittleren Elementkonzentrationen $C_{i}$ der vom $\mathrm{Ti}_{3} \mathrm{SiC}_{2}$-Target deponierten Schichten ohne Ionenstrahl (offene Symbole) und mit dem zugeschaltetem Argonionenstrahl mit $200 \mathrm{~V}$ Beschleunigungsspannung (geschlossene Symbole) sind als Funktion der Substrattemperatur in Abbildung 6.2 gezeigt. Abhängig von den Depositionsparametern schwankt der Titangehalt zwischen 49 und 56 at.-\%. Der Kohlenstoffanteil beträgt 28-34 at.-\%, der Siliziumgehalt liegt bei lediglich 7-12 at.-\%. Der jeweils zu $100 \%$ fehlende Anteil ist Sauerstoff und liegt im Bereich von 3-12 at.-\%. Der Sauerstoffgehalt bleibt bei Raumtemperatur (RT) und $450{ }^{\circ} \mathrm{C}$ in etwa gleich, steigt bei $550{ }^{\circ} \mathrm{C}$ zu einem Maximum an und fällt bei $650{ }^{\circ} \mathrm{C}$ sogar noch unter die ersten beiden Werte ab. Generell enthalten die mit Ionenstrahl hergestellten Schichten mehr Sauerstoff als die Proben, die ohne Ionenstrahl deponiert wurden. Verbunden mit dem lokalen Maximum des Sauerstoffgehalts bei $550{ }^{\circ} \mathrm{C}$ ist - besonders bei der Deposition mit Ionenstrahl - ein lokales Minimum des Titananteils in der Schicht. Die Konzentration des Siliziums nimmt in geringem Maße kontinuierlich mit steigender Substrattemperatur $\mathrm{ab}$, während der Kohlenstoffgehalt bei $\mathrm{T}=650^{\circ} \mathrm{C}$ am größten ist. Die Ursache für das lokale Maximum des Sauerstoffgehaltes bei $550^{\circ} \mathrm{C}$ ist nicht ganz klar. Das lokale Minimum der Titankonzentration bei $550{ }^{\circ} \mathrm{C}$ deutet allerdings auf eine verstärkte Oxidation des Titans hin. Bei $650{ }^{\circ} \mathrm{C}$ erreicht die Kohlenstoffkonzentration der Proben ihr Maximum. Die Kohlenstoffaufnahme in die Schicht durch Carbidbildung wird als Konkurrenzreaktion zur Oxidation bei diesen hohen Temperaturen also effektiver und verringert dadurch die Sauerstoffaufnahme.

Obwohl man, wie in Abschnitt 2.2 beschrieben, davon ausgehen kann, dass das Targetmaterial während der Ablation kongruent verdampft wurde, kam es offensichtlich nicht zum kongruenten Übertrag in die Schicht. Als Gründe für den nichtstöchiometrischen Elementübertrag vom Target in die Schicht kommen mehrere Effekte in Frage. Erreichen die ablatierten Partikel die Oberfläche der gerade wachsenden Schicht, unterliegen sie einer Vielzahl von Wechselwirkungen. Neben der Implantation in das Substratmaterial, bzw. die schon gebildete dünne Schicht, sind dies Oberflächenprozesse nach der Deposition (Diffusion, Nukleation, aber auch Abdampfen) und ein Wiederabsputtern von der Filmoberfläche durch Stöße mit den ankommenden Teilchen hoher kinetischer Energie. Eine übersichtliche Zusammenfassung zu den verschiedenen Effekten während der PLD wurde von H.-U. Krebs in [136] geliefert. Wie Fähler et al. zeigten, kann das Wiederabsputtern bei Metallen die Menge des tatsächlich deponierten Materials durchaus stark beeinflussen [137]. Es finden also konkurrierende Prozesse aus Deposition/Implantation und Abdampfen/Absputtern statt, aus denen sich in einem dynamischen Gleichgewicht letztendlich eine tatsächliche Zusammensetzung der dünnen Schicht ergibt. Da diese Konkurrenzprozesse für unterschiedliche Elemente - wie zum Beispiel hier Titan und Silizium verschieden groß sind, bestimmte Elemente also beispielsweise stärker abgesputtert werden oder abdampfen als andere, kommt es folglich zu Unterschieden in der chemischen 


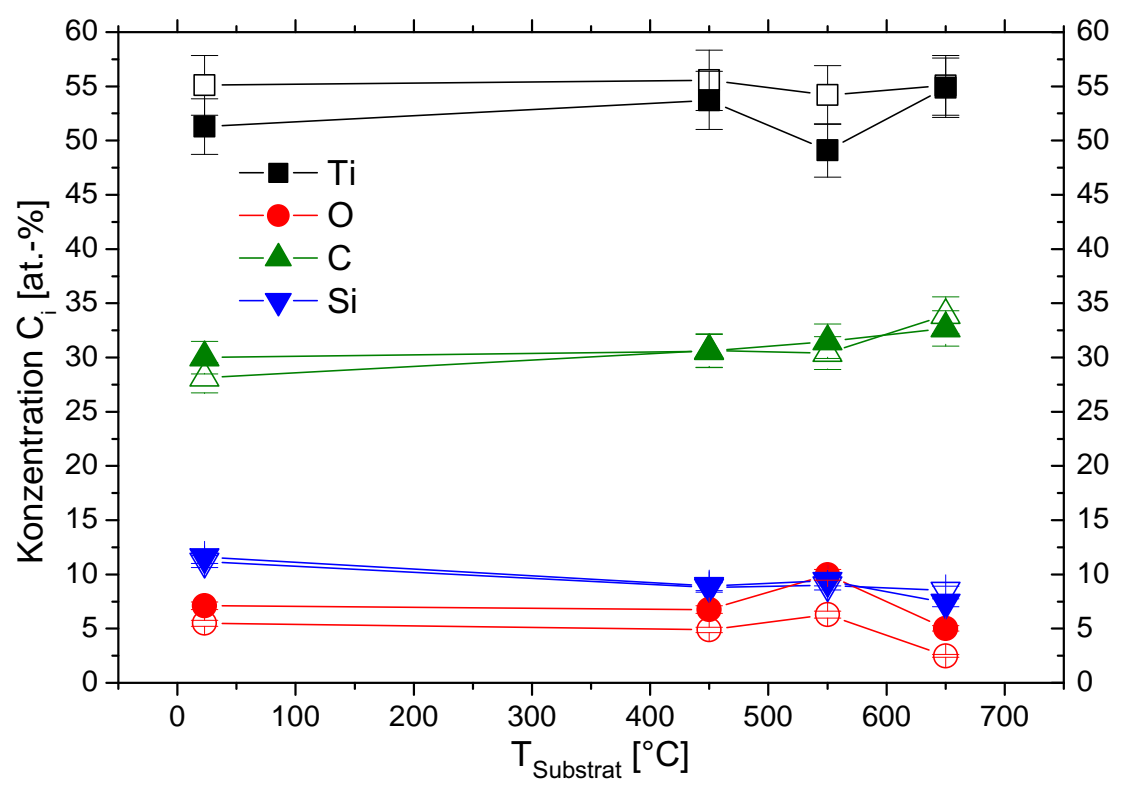

Abbildung 6.2: Mittlere Elementkonzentrationen $C_{i}$ der vom $\mathrm{Ti}_{3} \mathrm{SiC}_{2}$-Target auf $\mathrm{MgO}$ deponierten Schichten in Abhängigkeit der Substrattemperatur während der Ablation. Offene Symbole: ohne Ionenstrahl, geschlossene Symbole: mit zugeschaltetem $200 \mathrm{~V}$ Ar-Ionenstrahl. Die Linien dienen der besseren Orientierung.

Zusammensetzung zwischen Target und Schicht.

Einen weiteren Einfluss auf den tatsächlichen Übertrag der Elemente vom Target in die Schicht haben chemische Reaktionen in der Gasphase oder auf bzw. in der deponierten Schicht. Im hier vorliegenden Fall ist dies speziell die starke Sauerstoffaufnahme in die Schichten durch Oxidation. Wie bereits erwähnt enthalten die auf $\mathrm{MgO}(100)$ abgeschiedenen Schichten 3-12 at.-\% Sauerstoff, dessen Konzentration dabei im einzelnen von der Substrattemperatur und der Verwendung des Argon-Ionenstrahls abhängt. Der relativ hohe Sauerstoffgehalt der Proben deutet darauf hin, dass der in der Kammer bei der Deposition ohne Ionenstrahl erreichte Vakuumdruck von $10^{-5} \mathrm{~Pa}\left(10^{-7} \mathrm{mbar}\right) \mathrm{zu}$ hoch ist, und Restsauerstoff in der Kammer die Proben oxidiert. Ähnliches gilt für die Deposition mit Ar-Ionenstrahl. Dabei herrschte in der Kammer zwar eine Argonatmosphäre mit $5 \cdot 10^{-2} \mathrm{~Pa}$. Allerdings betrug die Reinheit des verwendeten Argons nur 99,998\%, so dass ein in der Größenordnung vergleichbarer Sauerstoffpartialdruck in der Kammer vorlag. Dazu kommt die kinetische Energie des Ionenstrahls von $200 \mathrm{eV}$, die durch oberflächennahe Diffusionsprozesse ebenfalls die Oxidation verstärken kann. Abbildung 6.3 zeigt den Vergleich der GDOES-Messungen der bei $650{ }^{\circ} \mathrm{C}$ ohne Ionenstrahl deponierten mit der bei $550{ }^{\circ} \mathrm{C}$ mit Ionenstrahl deponierten Schicht. Während Silizium und Kohlenstoff relativ 
homogen in der Schicht verteilt sind, ändern sich die Konzentrationen von Titan und Sauerstoffs mit der Schichttiefe. Da eine im Diagramm geringere Probentiefe in der Realität einer späteren Depositionszeit entspricht, kann also im Verlauf der Abscheidung zunehmend mehr Sauerstoff in die Filme inkorporiert werden. Da die Sauerstoffkonzentration zur Schichtoberfläche hin aber wieder abnimmt, erfolgt diese Sauerstoffaufnahme nicht nur direkt beim Wachstum der Schicht, sondern auch leicht verzögert durch Diffusion. Diese stärker werdende Oxidation lässt sich vermutlich durch die kristalline Mikrostruktur der Schichten erklären, und ist in Abschnitt 6.1.4.1 noch einmal ausführlicher dargestellt, da sich dieser Effekt bei den auf Edelstahl deponierten Schichten am deutlichsten zeigt.
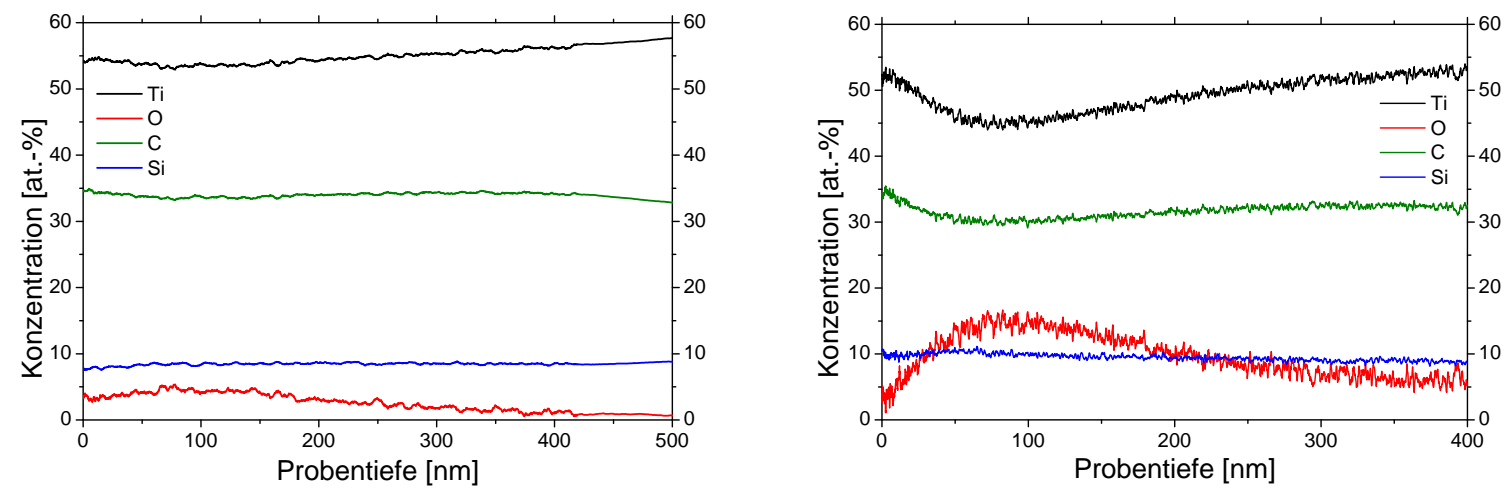

Abbildung 6.3: GDOES-Messungen zweier auf $\mathrm{MgO}(100)$ deponierter $\mathrm{Ti} / \mathrm{Si} / \mathrm{C}-\mathrm{Schichten}$. Links: $\mathrm{T}=650^{\circ} \mathrm{C}$, ohne Ionenstrahl, rechts: $\mathrm{T}=550^{\circ} \mathrm{C}$ mit $200 \mathrm{~V}$ Ar-Ionenstrahl. Die Elemente Si und C sind relativ homogen verteilt. Der Sauerstoff- und der Titananteil verändern sich mit der Probentiefe.

Mögliche chemische Reaktionen sind nicht nur wegen des Einbaus unerwünschter Verunreinigungen von Belang, sondern auch wegen der möglichen Bildung flüchtiger Molekülspezies, durch die es ebenfalls zu einem selektiven Verlust von Elementen während der Deposition kommen kann. Im Fall der Deposition vom $\mathrm{Ti}_{3} \mathrm{SiC}_{2}$-Target wäre eine solche Reaktion die Bildung von flüchtigem Siliziummonoxid SiO. Mögliche Reaktionen zur Entstehung von $\mathrm{SiO}$ wären die Oxidation von Silizium und die Reduktion von Siliziumdioxid $\mathrm{SiO}_{2}$

$$
\begin{aligned}
& 2 \mathrm{Si}+\mathrm{O}_{2} \rightarrow 2 \mathrm{SiO} \uparrow \\
& \mathrm{SiO}_{2}+\mathrm{Si} \rightarrow 2 \mathrm{SiO} \uparrow
\end{aligned}
$$

Diese Reaktionen können sowohl in der Gasphase und auf dem Substrat auftreten, und durch die Bildung von $\mathrm{SiO}$ lässt sich ein selektiver Verlust von Silizium in den Schichten 
ebenfalls erklären. Ein auf diesen Reaktionen zu SiO beruhendes Modell für die Verflüchtigung von Silizium aus der MAX-Phase $\mathrm{Ti}_{3} \mathrm{SiC}_{2}$ beschrieben auch Emmerlich et al. in [56]. Bei dem in den Schichten befindlichen Kohlenstoff können ebenfalls Oxidationen zu Kohlenmonoxid ( $\mathrm{CO})$ und -dioxid $\left(\mathrm{CO}_{2}\right)$ auftreten. Die GDOES-Ergebnisse zeigen jedoch, dass der Kohlenstoffverlust während der Abscheidung insgesamt nicht so hoch ist, wie der des Siliziums. Grund dafür ist die Tatsache, dass der Kohlenstoff schon während des Schichtwachstums durch Carbidbildung gebunden und damit einer möglichen Oxidationsreaktion entzogen wird.

Wie aus Abbildung $6.2 \mathrm{zu}$ erkennen ist, hat auch die Temperatur einen leichten Einfluss auf die Gesamtzusammensetzung der Schichten. Dieser lässt sich in diesem Fall aber gut durch die oben besprochenen chemischen Reaktionen erklären. Der zunehmende Verlust von Silizium kann durch eine zunehmende Oxidation zu SiO erklärt werden, der leicht ansteigende Kohlenstoffgehalt mit einer verstärkten Carbidbildung bei höheren Temperaturen, durch die der Kohlenstoff besser in der Schicht gehalten wird.

\subsubsection{Röntgendiffraktometrie und TEM-Messungen}

Die einzige kristalline Phase, die in den Röntgenbeugungsmessungen identifiziert werden konnte, ist kubisches Titancarbid TiC. Indiziert sind die TiC-Reflexe anhand des ICSD-Datenblattes Nr. 32-1383. Reflexe, die eindeutig der MAX-Phase $\mathrm{Ti}_{3} \mathrm{SiC}_{2}$ zugeordnet werden können, sind nicht zu erkennen. Die Abbildungen 6.4 (ohne Ionenstrahl) und 6.5 (mit Ionenstrahl) zeigen die Röntgendiffraktogramme der hergestellten Schichten bei allen Temperaturen. Gemessen wurde bei $5^{\circ}$ streifendem Einfall (GI), einer Schrittweite von $0,02^{\circ}$ und 1 Sekunde Messdauer pro Schritt. Aussagekräftiger wären Messungen in der $\theta / 2 \theta$-Geometrie. Im vorliegenden Fall sind die Schichten aber so dünn, dass mit dem zur Verfügung stehenden Messgerät auf die deutlich oberflächensensitivere GI-Messung ausgewichen werden musste.

In allen Proben liegt kristallines TiC vor. Die TEM-Aufnahmen (s.unten) zeigen, dass die Schichten dabei aus einem Gefüge von deutlichen kristallinen und amorphen Bereichen bestehen. Mit steigender Depositionstemperatur werden während der Messungen mehr Beugungsreflexe erkennbar. Bei Raumtemperatur (RT) wurde nur der TiC(200)-Reflex gemessen, der auch bei Messungen polykristalliner Pulver die stärkste Intensität besitzt. Bei $450^{\circ} \mathrm{C}$ treten zusätzlich die $\mathrm{TiC}(220)$ - und $\mathrm{TiC}(311)$-Reflexe auf, und erst bei $550^{\circ} \mathrm{C}$ wird auch die Beugung an TiC(111) erkennbar. Bei den Proben ohne Ionenstrahl ändern sich mit der Substrattemperatur die relativen Intensitäten der einzelnen Reflexe. Das Signal von $\mathrm{TiC}(111)$ wird stärker, während jenes von $\mathrm{TiC}(200)$ schwächer wird. Dieses könnte auf eine Änderung der kristallinen Vorzugsrichtung beim Wachstum der Schichten hindeuten. Da lediglich die Substrattemperatur geändert wurde, wäre dies ein thermisch 


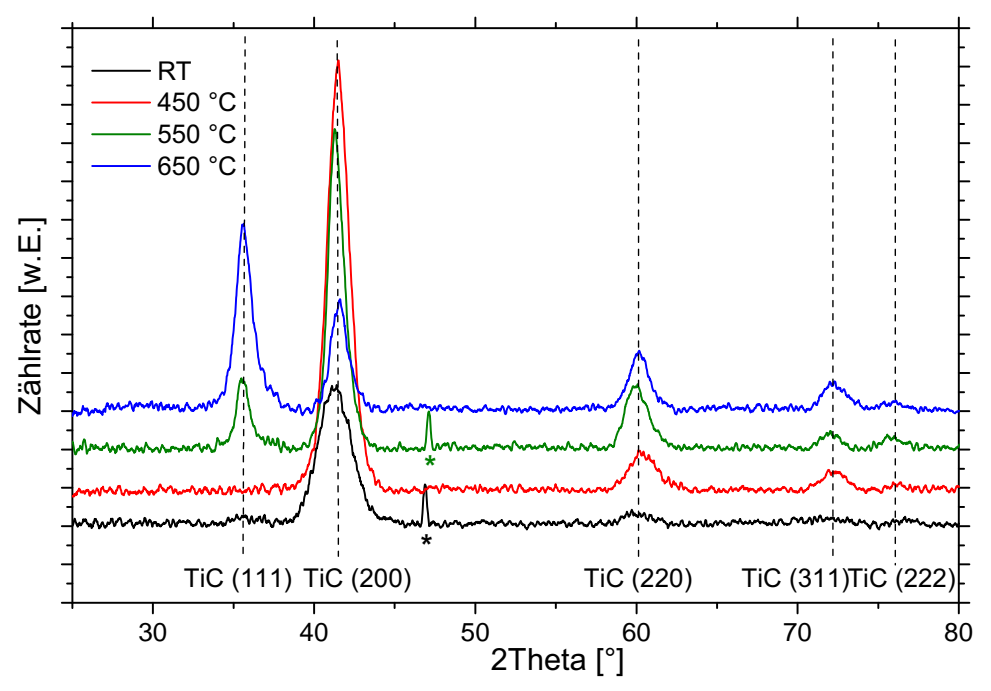

Abbildung 6.4: Röntgendiffraktogramme der Proben auf $\mathrm{MgO}(100)$ ohne Ionenstrahl. Indiziert sind die Reflexe für kubisches TiC. Die scharfen mit einem Stern markierten Reflexe sind Messartefakte, die vermutlich vom einkristallinen MgO-Substrat stammen.

gesteuerter Prozess, wie er für TiN auf $\mathrm{MgO}(100)$ in [121] diskutiert wurde (s. Abschnitt 2.2.4). Auch Wolfe et al. beschrieben bei der reaktiven Elektronenstrahlverdampfung von Titan eine Abhängigkeit der kristallographischen Vorzugsrichtung dünner TiC-Schichten von der Substrattemperatur [138]. Aus den in Abschnitt 3.1 dargestellten Gründen lassen sich aber allein aus GI-Messungen keine eindeutigen Rückschlüsse auf Vorzugsrichtungen oder Texturen ziehen. Die Elektronenbeugungsmessungen am TEM (s. unten) zeigen aber eine leichte kristallographische Vorzugsorientierung der Schichten an.

Die mit Ionenstrahl hergestellten Proben zeigen qualitativ ein vergleichbares Verhalten. Allerdings sind die Reflexe insgesamt weniger deutlich und auch weniger scharf, was auf eine geringere kristalline Qualität, d.h. kleinere Kristallite, der Schichten hindeutet. Eine Veränderung der relativen Intensitäten, wie bei den Proben ohne Ionenstrahl beobachtet, tritt nicht auf. Der Ionenbeschuss während der Deposition scheint also die Gesamtenergie des wachsenden Systems so zu beeinflussen, dass der rein thermische Effekt, der zur oben erwähnten Umkehrung der relativen Beugungsintensitäten führt, durch die kinetische Energie der eingestrahlten Argonionen überlagert wird. Außerdem ist es möglich, dass durch den Ionenstrahl induzierte andere Vorzugsrichtungen im Kristall auftreten als bei der Abscheidung ohne Ionenstrahl. Aufgrund des besonderen Streuvektorverhaltens in GI-Aufnahmen können diese aber wiederum nicht eindeutig erfasst werden.

Transmissionselektronenmikroskopische (TEM) Messungen bestätigen die Ergebnisse der Röntgenbeugungsmessungen. Abbildung 6.6 zeigt die TEM-Aufnahme eines Ausschnitts 


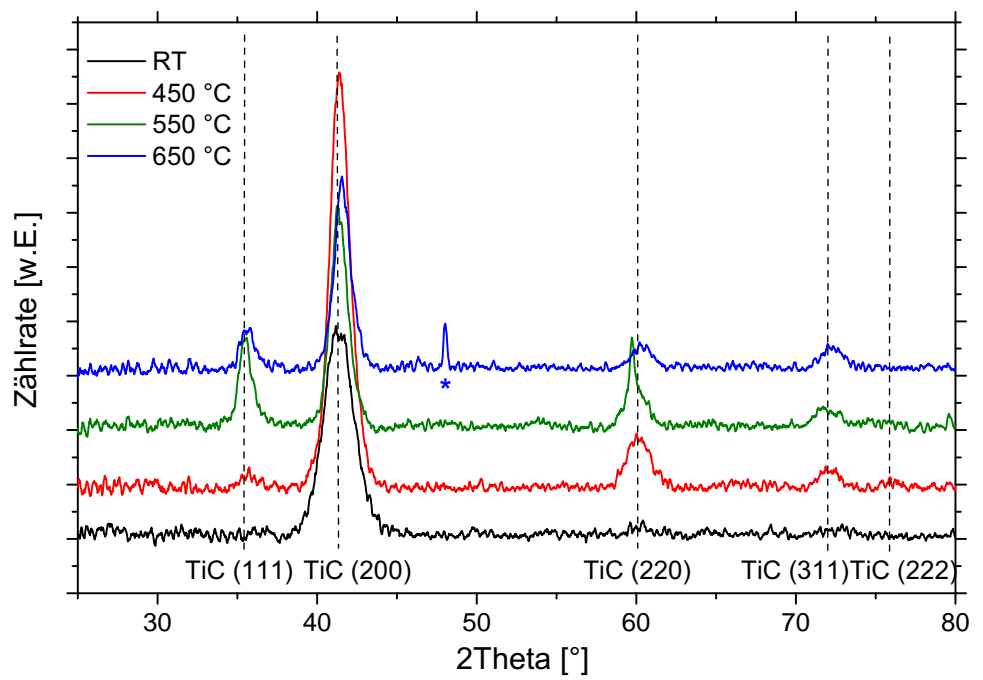

Abbildung 6.5: Röntgendiffraktogramme der Proben auf $\mathrm{MgO}(100)$ mit $200 \mathrm{~V}$ Ar-Ionenstrahl. Indiziert sind die Reflexe für kubisches TiC. Der scharfe mit einem Stern markierten Reflex stammt vermutlich vom einkristallinen MgO-Substrat.

aus der bei $650^{\circ} \mathrm{C}$ ohne Ionenstrahl auf $\mathrm{MgO}$ deponierten Probe. $\mathrm{Zu}$ sehen ist ein Gefüge aus polykristallinen und amorphen Bereichen in der Schicht. Im rechten Teil der Abbildung ist die schnelle Fourier-Transformation (FFT) des Mikroskopbildes dargestellt. In ihr lassen sich einzelne diskrete Punkte erkennen, die entsprechenden Netzebenenabständen zugeordnet werden können. Wie in Tabelle 6.1 beschrieben, entsprechen im Rahmen der Messgenauigkeit die meisten dieser Abstände denen von Titancarbid TiC. Dabei ist ebenfalls zu beachten, dass die relativ kleinen Kristallite in der Schicht erheblichen Spannungen ausgesetzt sein können, und die berechneten Abstände nicht genau den angegebenen theoretischen Werten entsprechen müssen.

Zwei der berechneten Netzebenenabstände können nicht dem TiC zugeordnet werden. Diese Werte entsprechen aber - wieder unter Betrachtung von Messgenauigkeit und intrinsischen Spannungen - dem (011)-Abstand im Titanmonoxid TiO (ICSD 23-1078). Diese Zuordnung wird durch die in Abschnitt 6.1.2.4 dargestellten XPS-Messungen bestätigt, in denen ebenfalls Titanoxid $\mathrm{TiO}_{x}$ nachgewiesen werden konnte.

Im Grenzbereich zwischen Substrat und Schicht wurden an der bei $650{ }^{\circ} \mathrm{C}$ ohne Ionenstrahl hergestellten Schicht außerdem Elektronenbeugungsmessungen (selected area electron diffraction, SAED) durchgeführt. Abbildung 6.7 zeigt die Ergebnisse dieser SAEDMessungen. Um eine bessere Erkennbarkeit zu ermöglichen, wurde das aufgenommene Beugungsbild schwarz/weiß-invertiert. Dunklere Bereiche entsprechen damit höheren Intensitäten. Im linken Teil der Abbildung ist die Beugungsmessung aus einem Bereich 
Tabelle 6.1: Netzebenenabstände aus der TEM-FFT-Rechnung der bei $650{ }^{\circ} \mathrm{C}$ ohne Ionenstrahl deponierten Schicht (siehe Abbildung 6.6).

\begin{tabular}{|c|c|c|}
\hline $\begin{array}{c}\text { gemessene } \\
\text { Netzebenenabstände (FFT) }\end{array}$ & $\begin{array}{c}\text { zugeordnete } \\
\text { Netzebenenschar }\end{array}$ & $\begin{array}{c}\text { theoretischer } \\
\text { Netzebenenabstand }\end{array}$ \\
\hline $0,2134 \mathrm{~nm}$ & $\operatorname{TiC}(200)$ & $0,2165 \mathrm{~nm}$ \\
$0,2138 \mathrm{~nm}$ & $\operatorname{TiC}(200)$ & $0,2165 \mathrm{~nm}$ \\
$0,2147 \mathrm{~nm}$ & $\operatorname{TiC}(200)$ & $0,2165 \mathrm{~nm}$ \\
$0,2489 \mathrm{~nm}$ & $\operatorname{TiC}(111)$ & $0,2500 \mathrm{~nm}$ \\
$0,3113 \mathrm{~nm}$ & $\operatorname{TiC}(110)$ & $0,3061 \mathrm{~nm}$ \\
\hline $0,3532 \mathrm{~nm}$ & $\operatorname{TiO}(011)$ & $0,3750 \mathrm{~nm}$ \\
$0,3650 \mathrm{~nm}$ & $\operatorname{TiO}(011)$ & $0,3750 \mathrm{~nm}$ \\
\hline
\end{tabular}
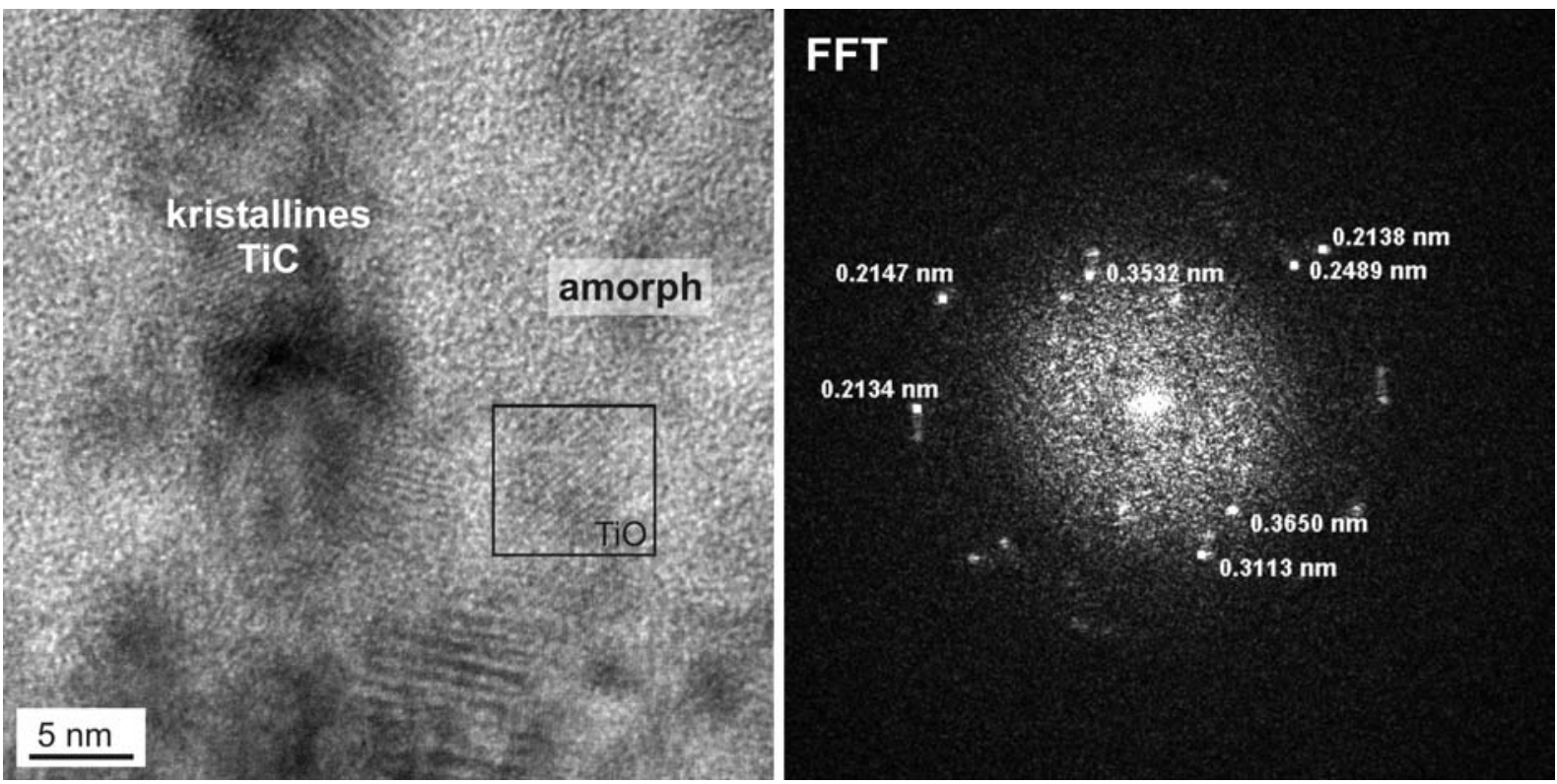

Abbildung 6.6: HR-TEM-Aufnahme der bei $650{ }^{\circ} \mathrm{C}$ ohne Ionenstrahl auf $\mathrm{MgO}$ deponierten Ti/Si/C-Schicht. Links: mikroskopisches Bild, rechts: schnelle Fourier-Transformation (FFT) des links dargestellten Bereiches. In der mikroskopischen Aufnahme sind deutlich sowohl polykristalline als auch amorphe Bereiche zu erkennen. Die aus der FFT berechneten Netzebenenabstände lassen sich $\mathrm{TiC}$ und $\mathrm{TiO}$ zuordnen.

innerhalb der Schicht zu sehen, im rechten Teil wurde dann direkt der Übergangsbereich zwischen Schicht und Substrat gemessen. Alle Beugungsintensitäten lassen sich dabei entweder TiC oder dem MgO-Substrat zuordnen. Die Messung aus dem Innern der Schicht zeigt eine polykristalline Struktur (Ringe) mit einer leichten vierzähligen Vorzugsorientierung (dunklere Bereiche in den Ringen). Im Übergangsbereich zwischen Substrat und Film ist die Vorzugsorientierung stärker ausgeprägt. Auffällig ist, dass die Beugungsintensitäten des TiC eine Art Schweif an den punktförmigen Reflexen des Substrats bilden. 
Die Vorzugsrichtung des $\mathrm{TiC}$ ist also nicht kongruent zu den Kristallrichtungen des $\mathrm{MgO}$ Substrats. Statt dessen kristallisiert das Titancarbid leicht verdreht - um etwa $20^{\circ}$ - auf dem Substrat. Unter den gegeben Depositionsparametern ist diese Form der Wachstumsausrichtung folglich energetisch begünstigt. Außerdem ist zu sehen, dass die Vorzugsrichtung in der Nähe des Substrates stärker ausgeprägt ist als in der Schicht. Es handelt sich also um einen Einfluss des Substrats auf das Schichtwachstum.
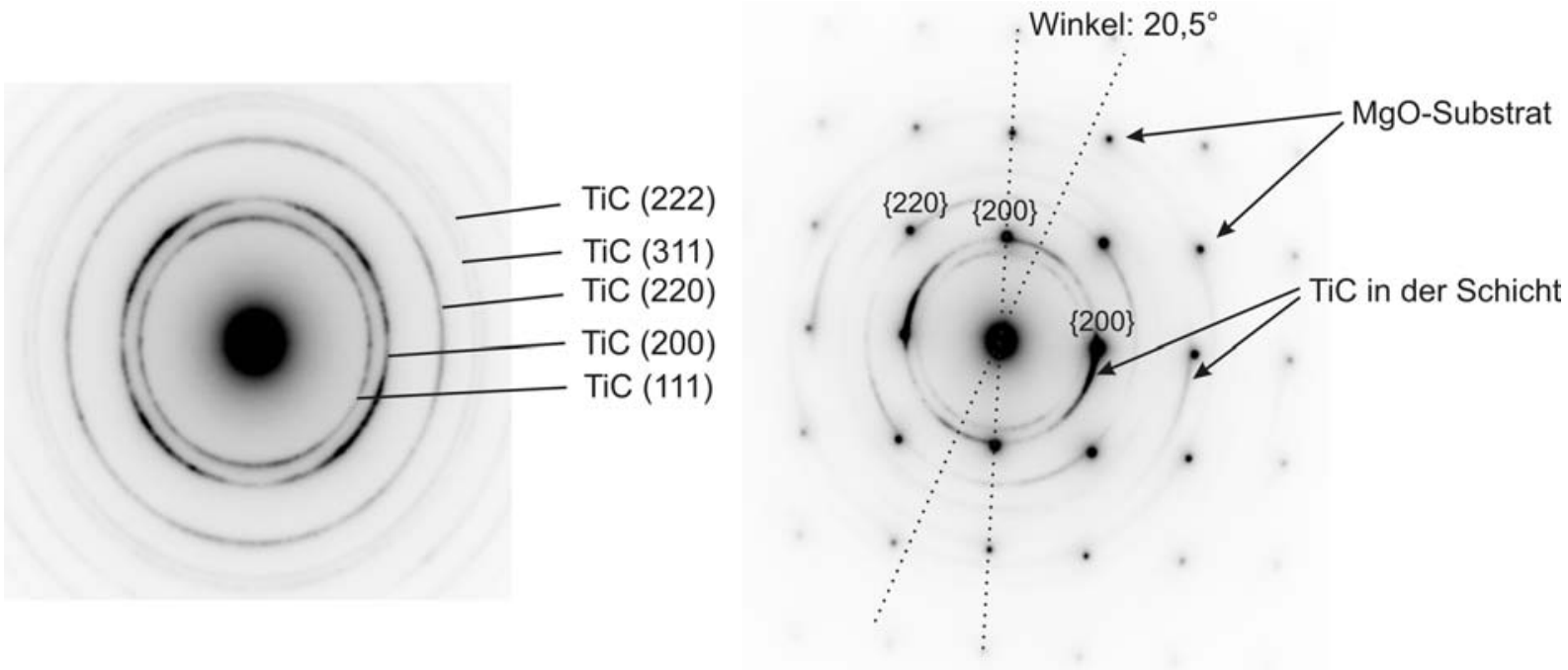

Abbildung 6.7: TEM-SAED-Messung der bei $650{ }^{\circ} \mathrm{C}$ ohne Ionenstrahl hergestellten Ti/Si/CSchicht (schwarz/weiß-invertiert). Links: Bereich aus dem Inneren der Schicht, rechts: Übergangsbereich zwischen Schicht und MgO-Substrat. Eine polykristalline TiC-Struktur mit leichter Vorzugsorientierung ist in der Schicht zu erkennen. Im Übergangsbereich ist die Vorzugsorientierung stärker ausgeprägt. Das $\mathrm{TiC}$ kristallisiert um etwa $20^{\circ}$ verdreht auf dem MgO-Substrat.

Diese Ergebnisse der Röntgenbeugungs- und TEM-Messungen sind in Übereinstimmung mit Ergebnissen aus der $\mathrm{Ti}_{3} \mathrm{SiC}_{2}$-MAX-Filmsynthese durch Magnetronsputtern. Erst bei Substrattemperaturen von $800-900{ }^{\circ} \mathrm{C}$ wurden dabei die MAX-Phasen gebildet [38, 55]. Bei darunter liegenden Temperaturen waren die Proben entweder röntgenamorph, oder es bildete sich - wie auch hier - eine $\mathrm{TiC}_{x}$-Matrix, in die das Silizium eingebettet war. Aufgrund der in Abschnitt 2.1.1 beschriebenen großen strukturellen Ähnlichkeit der MAXPhasen mit den binären MX-Verbindungen ist dies das zu erwartende Ergebnis, wenn sich das A-Element Silizium nicht entsprechend der MAX-Stapelfolge in die $\mathrm{TiC}_{x}$-Matrix einbaut. Eine genauere Aussage über die chemischen Verhältnisse in der Probe - insbesondere über die Umgebungen von Silizium und Sauerstoff - lässt sich durch die Röntgenbeugungsmessungen nicht treffen, da keine weitere Phase außer TiC identifiziert werden konnte. Aus diesem Grund wurden an einigen wenigen auf Silizium deponierten Schichten XPSMessungen durchgeführt, die in Abschnitt 6.1.2.4 beschrieben werden. Deren Ergebnisse zeigen außer $\mathrm{TiC}$ auch noch die Existenz von geringen Mengen des Siliziumcarbid SiC, 
sowie der Oxide $\mathrm{TiO}_{x}$ und $\mathrm{SiO}_{x}$.

\subsubsection{Härtemessungen}

Die gemessenen Härtewerte der Proben (s. Abb. 6.8) liegen - abhängig von den Depositionsparametern - in einem Bereich von 8-13 GPa. Verglichen mit den Härten dünner $\mathrm{Ti}_{3} \mathrm{SiC}_{2^{-}}$und dünner TiC-Schichten ist dies relativ weich. So wurden für dünne TiCSchichten Härtewerte von 15-25 GPa [139] bzw. sogar über 30 GPa [140] beschrieben. Für Filme aus $\mathrm{Ti}_{3} \mathrm{SiC}_{2}$-MAX-Phasen wurden bei kleinen Prüfkräften Härten von 15-30 GPa gemessen $[38,40]$, die bei höheren Prüfkräften und größeren Eindringtiefen allerdings auf die Werte des $\mathrm{Ti}_{3} \mathrm{SiC}_{2}$-Vollmaterials (4-6 GPa) zurück gingen, da in diesem Fall dann die in Abschnitt 2.1.1 beschriebenen besonderen Deformationsmechanismen - insbesondere die Ausbildung von Knickbändern - von MAX-Phasen auftraten.

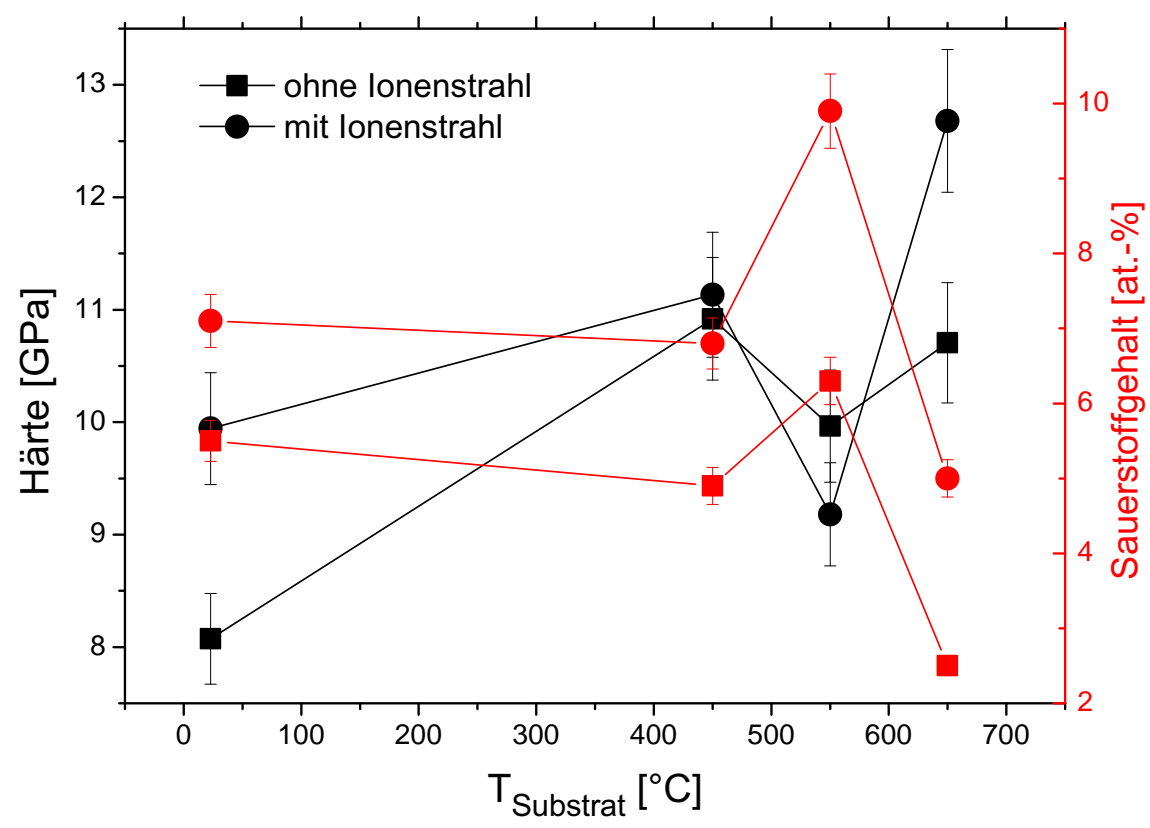

Abbildung 6.8: Schichthärten (schwarz) und Sauerstoffgehalt (rot) der auf $\mathrm{MgO}(100)$ deponierten Proben (Quadrate: ohne Ionenstrahl, Kreise: mit Ionenstrahl). Die Linien dienen der besseren Orientierung.

Bei den ohne Ionenstrahl deponierten Proben nimmt die Schichthärte von etwa 8 GPa bei Raumtemperatur auf 10-11 GPa bei geheiztem Substrat zu. Die mit Ionenstrahl hergestellten Schichten sind allerdings im Vergleich meist härter. Hier liegen die gemessenen Werte zwischen etwa $10 \mathrm{GPa}$ bei Raumtemperatur und fast $13 \mathrm{GPa}$ bei $650{ }^{\circ} \mathrm{C}$. 
Obwohl die Messung der Schichthärten relativ einfach ist, und die Zahlenwerte der Ergebnisse schnell gewonnen sind, ist eine Interpretation dieser Werte nicht trivial. Denn die Härte der Schichten ist abhängig von vielen Parametern. Den größten Einfluss hat dabei die kristalline Mikrostruktur der Schicht. Die hier gemessenen Schichten bestehen wie oben beschrieben aus eine Gefüge von amorphen und kristallinen Bereichen, wobei die Kristallite Größen im unteren Nanometerbereich aufweisen. Dieser Größenbereich ist bei der Betrachtung der Schichthärte insofern interessant, als dass es hier zu einer Erhöhung der Materialhärte durch die nanoskalige Größe der Kristallite kommen kann. Dieser Effekt der Materialhärtung durch Verkleinerung der Kristallitgröße beruht auf der zunehmenden Unterdrückung der Bewegung von Versetzungen durch mehr Korngrenzen. Die unterschiedlichen Körner haben unterschiedliche kristallographische Orientierungen, so dass es mehr Energie erfordert, die Bewegung einer Gitterversetzung von einem Korn in das andere fortzusetzen. Mathematisch beschrieben wird dieser Effekt durch die HallPetch-Beziehung [141, 142]:

$$
\sigma_{y}=\sigma_{0}+k \frac{1}{\sqrt{d}}
$$

Dabei sind $\sigma_{y}$ die so genannte Streckgrenze - d.h. die Grenze, bis zu der der Werkstoff bei mechanischer Belastung keine plastische Verformung zeigt,$- \sigma_{0}$ die Belastung, die notwendig ist, um eine Versetzungsbewegung im Material hervorzurufen, $k$ der Härtungskoeffizient (eine materialabhängige Konstante) und $d$ der mittlere Durchmesser der Kristallite. Diese Beziehung wurde ursprünglich für die mechanischen Belastungsgrenzen von Vollmaterial aufgestellt. Sie lässt sich aber auch auf die Kristallitgrößen in dünnen Schichten anwenden (vgl. [58, 143]). Theoretisch ließe sich nach der Hall-Petch-Gleichung bei unendlichen kleinen Kristalliten ein unendlich hartes Material erreichen. Tatsächlich ist dies aber nicht der Fall, denn unterhalb einer kritischen Kristallitgröße $d_{c}$ tritt ein anderer Mechanismus zum Abbau von mechanischer Belastung auf. Die Korngrenzen gleiten nun gegeneinander ab, und die Härte des Materials verringert sich wieder [144, 145]. Die kritische Kristallitgröße ist dabei eine materialabhängige Größe und liegt typischerweise in Bereich von einigen zehn Nanometern [143, 146, 147].

Liegen in der Schicht mehrere Phasen vor, und treten dadurch Inhomogenitäten im Gefüge auf, beeinflusst dies auch die Schichthärte, und die Schichten werden weicher. Im Fall der hier untersuchten Proben ist dies die Bildung oxidischer Phasen durch den Sauerstoffeinbau in die Schichten, die weiter unten diskutiert wird.

In den hier beschriebenen Proben tritt zunächst eine Zunahme der Schichthärte bei steigender Substrattemperatur auf. Wie die Röntgenbeugungsmessungen zeigen, nimmt dabei die Größe der Kristallite zu. Das Abgleiten der Korngrenzen in Bereichen sehr kleiner Körner nimmt ab, und die Schichten werden härter. Bei Raumtemperatur und $650{ }^{\circ} \mathrm{C}$ sind 
die mit dem Ionenstrahl beschossenen Proben messbar härter, obwohl sich in den Röntgendiffraktogrammen nicht unbedingt eine höhere Kristallinität der Schichten erkennen lässt. Die Kristallitgrößen liegen aber im Bereich von $d_{c}$, so dass in den Proben eine Konkurrenz zwischen hart (Kristallite) und weich (amorphe Bereiche und Kristallite $<d_{c}$ ) auftritt. Im Fall der bestrahlten RT- und $650{ }^{\circ} \mathrm{C}$-Proben liegt dieses Gleichgewicht offensichtlich mehr in Richtung hart als bei den unbestrahlten Proben.

Wie oben bereits angemerkt, tritt bei den Härtewerten für beide Probenarten bei $550{ }^{\circ} \mathrm{C}$ ein lokales Minimum auf, also genau dort, wo der Sauerstoffgehalt jeweils ein lokales Maximum aufweist. In Abbildung 6.8 ist dieser Sachverhalt illustriert. Der Sauerstoffgehalt bildet folglich - neben Substrattemperatur und Ionenbeschuss - eine weitere Einflussgröße auf die Schichthärte. Offensichtlich wird die Struktur der harten carbidischen TiC-Matrix durch den Einbau oxidischer Verbindungen erheblich gestört, so dass es zu einer Verringerung der Schichthärte kommt. Ein vergleichbarer Zusammenhang zwischen steigendem Sauerstoffgehalt und sinkender Schichthärte in dünnen TiC-Schichten wurde zum Beispiel auch von Lopez et al. bei der Ionenstrahldeposition von TiC-Pulver festgestellt [148].

Einen dritten Einfluss auf die hier gemessenen Größen kann auch die Härte des Substrates, auf die die Schichten deponiert wurden, haben. Obwohl die Prüftiefe in keinem Fall etwa $30 \%$ der Schichtdicke übertraf, können die auftretenden Kräfte bei der Härteprüfung durchaus längere Reichweiten haben. Diese Kräfte reichen dann in das Substratmaterial hinein, so dass sich nicht ausschließen lässt, dass auch dessen Härte in die gemessene Gesamthärte mit eingeht. Die Härte der MgO-Substrate wurde in dieser Arbeit mit $H_{\mathrm{MgO}}$ $\approx 10,0 \mathrm{GPa}$ gemessen. 


\subsubsection{Si(100)-Substrat}

Um den relativ geringen Siliziumgehalt der deponierten Proben durch Diffusionsprozesse eventuell anheben zu können, wurden Schichten aus dem $\mathrm{Ti}_{3} \mathrm{SiC}_{2}$-Target ebenfalls auf einkristallines $\operatorname{Si}(100)$ deponiert. Wie bereits oben erwähnt, entstanden dabei wiederum homogene rissfreie Filme. Lediglich bei der Deposition bei $650{ }^{\circ} \mathrm{C}$ mit dem Ionenstrahl riss die Schicht bereits während der Deposition aufgrund von intrinsischen Spannungen. Aus diesem Grund gibt es für diese Probe in den folgenden Abschnitten keine Messwerte.

\subsubsection{GDOES-Messungen}

Wie auch bei der Deposition auf MgO-Substraten wurde die stöchiometrische Zusammensetzung des $\mathrm{Ti}_{3} \mathrm{SiC}_{2}$-Targets in den Proben nicht erreicht. Die mittlere Konzentration von Titan liegt zwischen 50 und 53 at.-\%, die von Kohlenstoff zwischen 28 und 33 at.- $\%$ und die von Silizium zwischen 9 und 11 at.-\%. Die zu $100 \%$ fehlenden Anteile werden wiederum von Sauerstoff gebildet und betragen 8-13 at.-\%. Die Gesamtzusammensetzungen aller Proben zeigt Abbildung 6.9. Sie entsprechen weitestgehend denen, die auch bei der Deposition auf $\mathrm{MgO}$ gemessen wurden. Lediglich der Sauerstoffgehalt ist um wenige Atomprozente höher. Es kommt folglich zu einer stärkeren Oxidation der Schichten. Die anderen qualitativen Merkmale, das heißt das lokale Sauerstoffmaximum und der Abfall des Sauerstoffgehaltes bei $650^{\circ} \mathrm{C}$ sind ebenfalls zu erkennen, allerdings weniger stark ausgeprägt. Die Diskussion zur chemischen Zusammensetzung der Schichten folgt damit der Argumentation, die für die MgO-Proben in Abschnitt 6.1.1.1 geführt wurde.

Abbildung 6.10 zeigt die GDOES-Messungen für die ohne und mit Ionenstrahl deponierten Schichten bei der jeweils höchsten Substrattemperatur. Wiederum sind die Elemente Si und C homogen in den Schichten verteilt, und wiederum ist auch der Sauerstoffanteil über die Probentiefe veränderlich, was in Abschnitt 6.1.4.1 genauer erläutert wird. Eine Diffusion von Silizium in die Schicht findet - auch bei Anwendung des Ionenstrahls - nicht statt. Allerdings ist zu erkennen, dass die ablatierten Atome zum Teil in das Siliziumsubstrat hinein diffundieren.

Insgesamt sind die auf Silizium deponierten Schichten also vergleichbar mit den auf MgO hergestellten Proben. Wie die XRD-Messungen im folgenden Abschnitt zeigen, unterscheiden sie sich lediglich in ihrer kristallinen Qualität. Dies ist vermutlich gleichzeitig auch der Grund für die höhere Sauerstoffaufnahme (s. Abschnitt 6.1.4.1).

\subsubsection{Röntgendiffraktometrie}

Die Ergebnisse der Röntgenbeugungsmessungen der auf Si(100)-Substraten hergestellten Schichten sind ebenfalls mit denen der $\mathrm{MgO}(100)$-Proben vergleichbar. Es handelt sich 


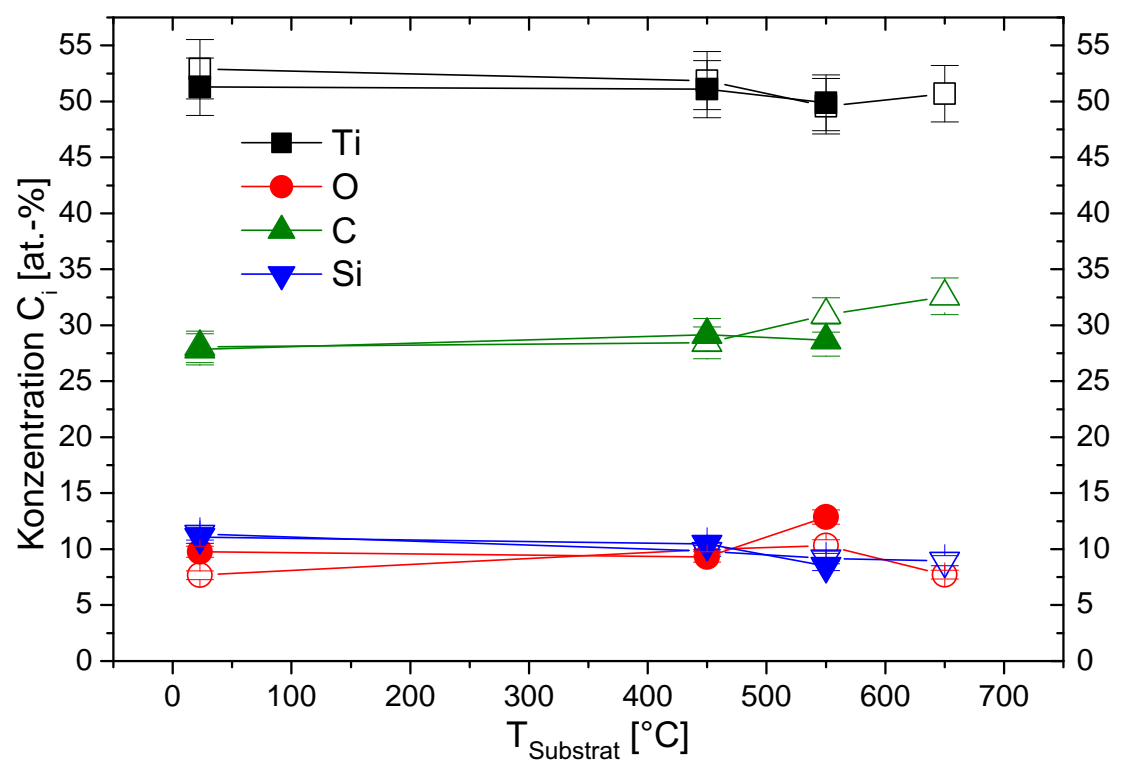

Abbildung 6.9: Mittlere Elementkonzentrationen $C_{i}$ der vom $\mathrm{Ti}_{3} \mathrm{SiC}_{2}$-Target auf $\mathrm{Si}$ deponierten Schichten in Abhängigkeit der Substrattemperatur während der Ablation. Offene Symbole: ohne Ionenstrahl, geschlossene Symbole: mit zugeschaltetem $200 \mathrm{~V}$ Ar-Ionenstrahl. Die Linien dienen der besseren Orientierung.
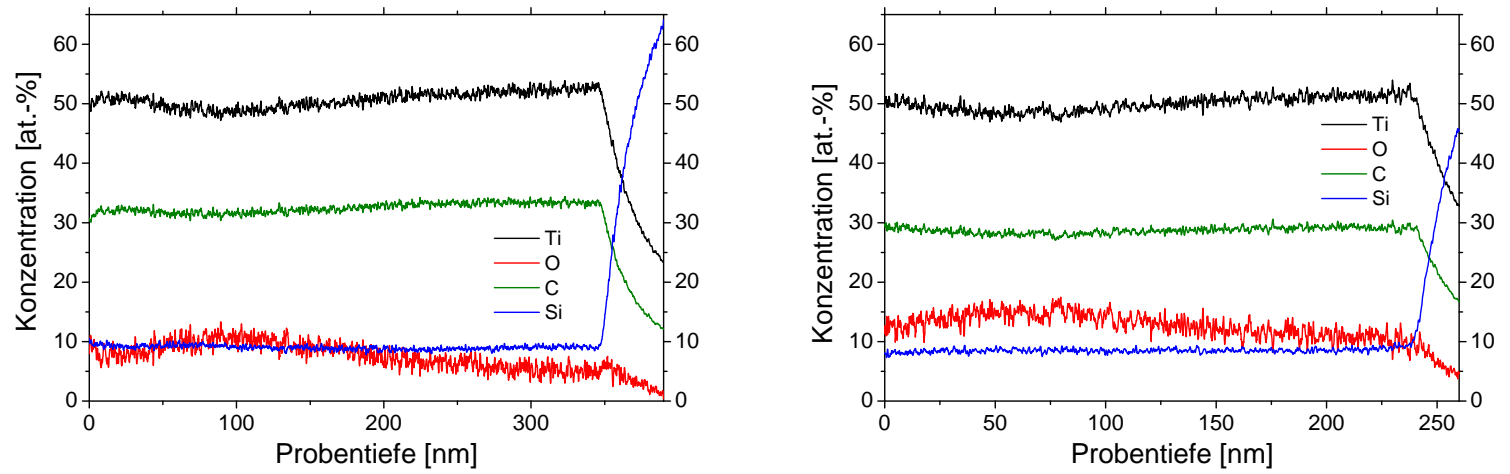

Abbildung 6.10: GDOES-Messungen zweier auf $\mathrm{Si}(100)$ deponierter Ti/Si/C-Schichten. Links: $\mathrm{T}=650^{\circ} \mathrm{C}$, ohne Ionenstrahl, rechts: $\mathrm{T}=550^{\circ} \mathrm{C}$ mit $200 \mathrm{~V}$ Ar-Ionenstrahl. Die Elemente Si und $\mathrm{C}$ sind homogen verteilt, aber die Titan- und Sauerstoffkonzentrationen ändern sich mit der Probentiefe. Eine Diffusion von Silizium in die Schichten findet nicht statt.

dabei um Messungen mit $5^{\circ}$ streifendem Einfall (GI) bei einer Schrittweite von $0,02^{\circ}$ und 1 Sekunde Messdauer pro Schritt. Aufgrund der geringen Schichtdicke war die Verwendung dieser Messgeometrie notwendig. Wiederum ist die einzige kristallographisch 
identifizierbare Phase kubisches Titancarbid TiC (s. Abbildungen 6.11 (ohne Ionenstrahl) und 6.12 (mit Ionenstrahl)). Bei Raumtemperatur ist auch hier nur der TiC(200)-Reflex erkennbar, während bei höheren Temperaturen die anderen Reflexe ( $\mathrm{TiC}(111), \mathrm{TiC}(220)$ und $\mathrm{TiC}(311))$ erscheinen. Die in Abschnitt 6.1.1.2 beschriebene Umkehrung der relativen Intensitäten von $\mathrm{TiC}(200)$ - und $\mathrm{TiC}(111)$-Reflex bei den ohne Ionenstrahl deponierten Schichten tritt auch auf dem Silizium-Substrat auf. Es kommt also wie auf den MgO-Substraten zu einer thermisch kontrollierten bevorzugten Ausrichtung der Kristallorientierungen während des Wachstums.

Die Reflexe der Proben mit Ionenstrahl sind - bis auf $\mathrm{TiC}(200)$ - weniger stark ausgeprägt als bei den Proben, die ohne Ionenstrahl hergestellt wurden. Eine Umkehrung der relativen Reflexintensitäten konnte erneut nicht festgestellt werden. Aufgrund der Ähnlichkeit der Ergebnisse mit denen der MgO-Proben lässt sich die Diskussion genau wie in Abschnitt 6.1.1.2 führen. Dabei gilt es, wie oben beschrieben auch, den für genauere Aussagen unzureichenden Informationsgehalt der verwendeten GI-Geometrie in Betracht zu ziehen.

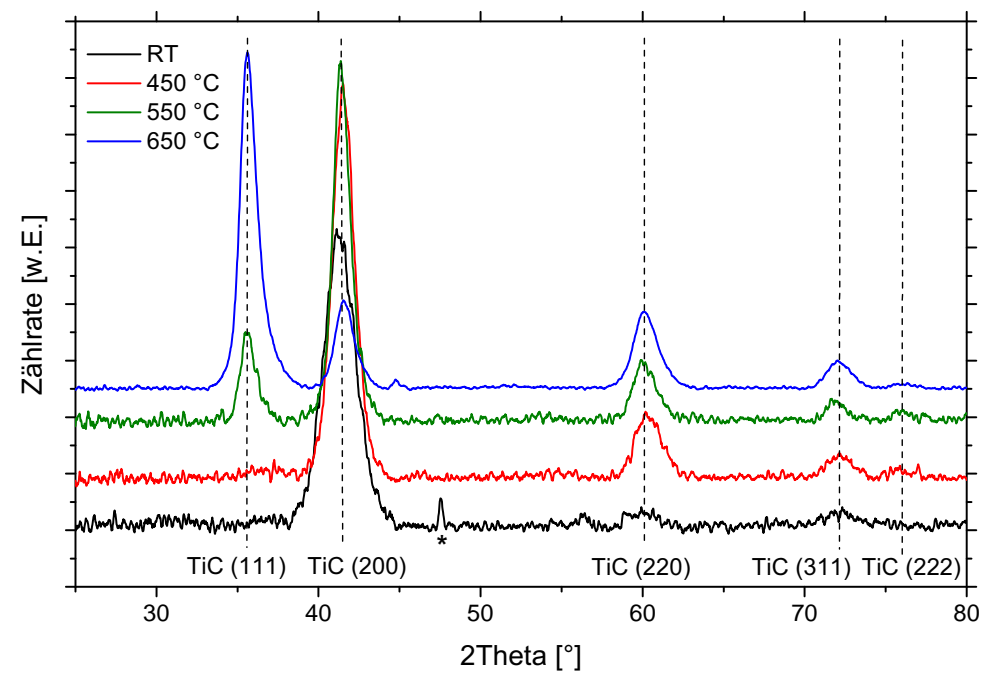

Abbildung 6.11: Röntgendiffraktogramme der Proben auf $\mathrm{Si}(100)$ ohne Ionenstrahl. Indiziert sind die Reflexe für kubisches TiC. Der scharfe mit einem Stern markierte Reflex ist vermutlich ein vom einkristallinen Si-Substrat stammendes Messartefakt.

\subsubsection{Härtemessungen}

Mit gemessenen Härten im Bereich von 7,2-9,6 GPa sind die auf Silizium deponierten Schichten weicher als diejenigen, die auf $\mathrm{MgO}$ abgeschieden wurden. Allerdings ist in den 


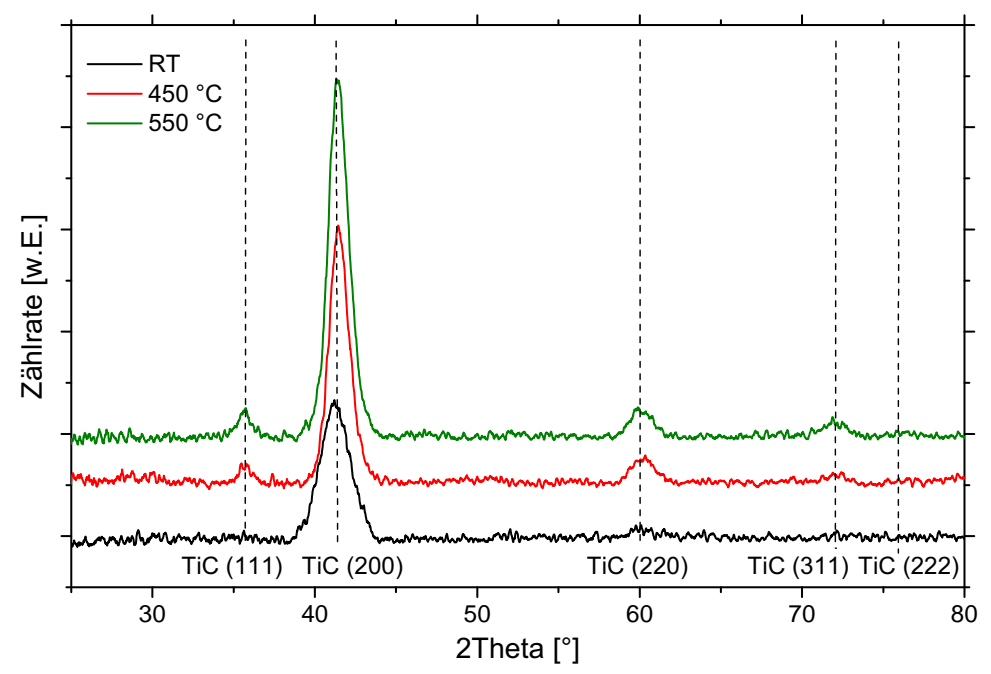

Abbildung 6.12: Röntgendiffraktogramme der Proben auf Si mit 200 V Ar-Ionenstrahl. Indiziert sind die Reflexe für kubisches TiC.

Proben auch die Sauerstoffkonzentration höher, weswegen entsprechend der Diskussion zu den $\mathrm{MgO}-$ Proben auch geringere Schichthärten erwartet werden können. Die entsprechenden Messwerte sind in der Grafik in Abbildung 6.13 dargestellt. Vergleicht man jedoch die Zahlen für Sauerstoffgehalt und Schichthärte mit denen der auf MgO deponierten Filme, stellt man fest, dass der höhere Sauerstoffanteil nicht allein der Grund für die geringeren Härten sein kann. So sind bei einem vergleichbaren Sauerstoffgehalt von etwa 10 at.-\% die auf $\mathrm{Si}(100)$ deponierten Schichten um etwa 2 GPa weicher als die Filme auf $\mathrm{MgO}(100)$. Es muss also noch einen Einfluss des Substrates auf die kristallinen Eigenschaften der Schicht und damit die Schichthärte existieren.

Betrachtet man allein die Si-Proben, so nehmen die Schichthärten bei höheren Substrattemperaturen im Vergleich zu Raumtemperatur zu. Aus den XRD-Messungen wird deutlich, dass dies auf eine größere Zahl kristalliner Bereiche in den Schichten zurück geführt werden kann. Auch sind - wie im Fall der Deposition auf $\mathrm{MgO}$ - die mit Ionenstrahl bei Raumtemperatur und $450{ }^{\circ} \mathrm{C}$ hergestellten Proben härter. Analog zur Deposition auf $\mathrm{MgO}$ kommt es offensichtlich wieder zu einer Beeinflussung der Schichthärte durch das Hall-Petch-Regime, die in den Röntgenbeugungsdiagrammen nicht unbedingt erkennbar ist. Bei $550{ }^{\circ} \mathrm{C}$ allerdings ist die mit Ionenstrahl hergestellte Schicht bedeutend weicher als die ohne Ionenstrahl deponierte Probe. Dies lässt sich durch den bedeutend höheren Sauerstoffanteil der Ionenstrahlprobe erklären (s. Abschnitt 6.1.1.3). Wie im Fall der Deposition auf MgO lässt sich auch ein Einfluss der Substrathärte auf die gemessene Gesamthärte nicht ausschließen. Verglichen mit den MgO-Substraten (ca. $10 \mathrm{GPa}$ ) sind die Siliziumsubstrate weicher $-H_{\mathrm{Si}} \approx 6,5 \mathrm{GPa}$. 


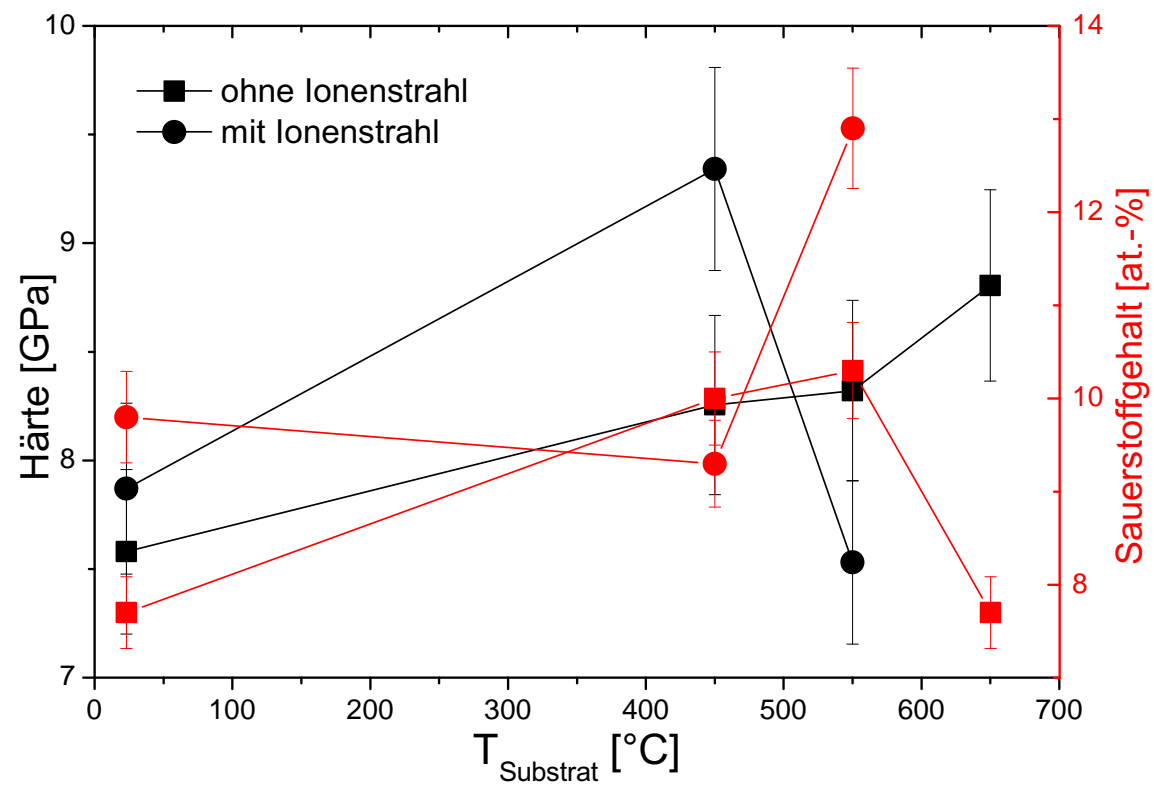

Abbildung 6.13: Schichthärten (schwarz) und Sauerstoffgehalt (rot) der auf $\operatorname{Si}(100)$ deponierten Proben (Quadrate: ohne Ionenstrahl, Kreise: mit Ionenstrahl). Die Linien dienen der besseren Orientierung.

\subsubsection{XPS-Messungen}

Um genauere Erkenntnisse über die chemischen Umgebungen der einzelnen Elemente in der Schicht zu gewinnen, wurden an wenigen Proben XPS-Messungen durchgeführt. Die XPS-Messungen erfolgten in Zusammenarbeit mit Prof. Ulf Jansson an der Universität Uppsala, Schweden. Da XPS eine Informationstiefe von nur wenigen Nanometern hat (s. Abschnitt 3.3), wurde die Oberfläche der Proben vor der Messung mit einem Argon-Ionenstrahl abgesputtert, um tatsächlich Informationen aus der Schicht gewinnen zu können. Im Verlauf der Arbeiten hat sich dabei herausgestellt, dass die XPS-Spektren durch die vom Ioneneinschlag hervorgerufenen Schäden beeinflusst werden können. Insbesondere die spektralen Linien von Kohlenstoff werden erheblich gestört [Prof. Jansson, persönliche Mitteilung]. Dadurch werden sehr viel intensivere Untersuchungen bei verschiedenen Sputterenergien notwendig, die im Rahmen der hier vorgelegten Arbeit nicht mehr erfasst werden können.

Im Folgenden sollen die Ergebnisse der ersten durchgeführten XPS-Messungen an auf $\mathrm{Si}(100)$ abgeschiedenen Ti/Si/C-Schichten vorgestellt werden. Die Deposition der Schichten erfolgte dabei ohne Ar-Ionenstrahl. Gemessen wurden die Bindungsenergiebereiche der Orbitale Ti 2p, Si 2p und C 1s. Vorgesputtert wurde für 60 Sekunden mit einem 4 kV 
$\mathrm{Ar}^{+}$-Strahl. Die Auswertung der einzelnen Peaks erfolgte mit der Software XPS Peak Version 4.1 [149]. Zur Interpretation der gemessenen Bindungsenergien wurde die XPSDatenbank NIST X-ray Photoelectron Spectroscopy Database (Version 3.5) des National Institute of Standards and Technology der USA genutzt [150]. Abbildung 6.14 zeigt die Messung des Ti 2p-Orbitals im Bereich der Bindungsenergien $E_{B}$ von 451-466 eV der bei Raumtemperatur deponierten Probe. Das Messspektrum wurde in fünf Peaks entfaltet, die zur Messung beitragen und folgenden Bindungen zugeordnet wurden:

- (1) $E_{B}=454,0 \mathrm{eV}: \mathrm{Ti} 2 \mathrm{p} \frac{3}{2}$ in $\mathrm{Ti}$

- (2) $E_{B}=454,9 \mathrm{eV}: \mathrm{Ti} 2 \mathrm{p} \frac{3}{2}$ in $\mathrm{TiC}$,

- (3) $E_{B}=456,6 \mathrm{eV}: \mathrm{Ti} 2 \mathrm{p} \frac{3}{2}$ in $\mathrm{TiO}_{1,5}$,

- (4) $E_{B}=460,0 \mathrm{eV}: \mathrm{Ti} 2 \mathrm{p} \frac{3}{2}$ in $\mathrm{Ti} / \mathrm{TiO}_{x}$ und

- (5) $E_{B}=460,5 \mathrm{eV}$ : Ti $2 \mathrm{p} \frac{3}{2}$ in $\mathrm{TiO}$.

In der Probe findet sich folglich Titan als reines Metall, als Titancarbid TiC und als Titanoxid $\mathrm{TiO}_{x}$ in unterschiedlichen Oxidationsstufen. Dabei bilden das metallische Titan und die Titanoxide allerdings keine großen kristallinen Bereiche, die den Röntgenbeugungsmessungen zugänglich sind. In den TEM-Aufnahmen hingegen wurden kleine TiO-Kristallite gefunden.

In Abbildung 6.15 ist die Messung des Si 2p-Orbitals in einem Bindungsenergiebereich von 95-107 eV dargestellt. In diesem Fall wurde die gemessene Kurve in vier Peaks entfaltet. Deren Zuordnung zu bestimmten Bindungen ist allerdings nicht in jeden Fall so eindeutig wie bei der Messung von Titan. Relativ eindeutig zuweisen lässt sich Peak $4 E_{B}=$ $101,0 \mathrm{eV}$ zu Si 2p $\frac{3}{2}$ in Siliziumcarbid SiC. Die Bindungsenergie von Peak $2 E_{B}=98,4 \mathrm{eV}$ entspricht laut Datenbank Si 2p in reinem Silizium. Für Peak $3 E_{B}=99,0 \mathrm{eV}$ findet sich in der Datenbank ein Eintrag für Si 2p von Si in einer $\mathrm{SiO}_{x}$-Matrix. Für die Bindungsenergie von Peak $1 E_{B}=97,9 \mathrm{eV}$ ist in der Datenbank keine Verbindung angegeben, die der chemischen Zusammensetzung der Probe aus Ti, Si, C und O entspricht. Grund dafür könnte der oben beschriebene Verzerrungseffekt durch das Sputtern mit den hochenergetischen Ionenstrahl sein. Dennoch lässt die Messung den Schluss zu, dass das Silizium in der Probe mehrere verschiedene chemische Umgebungen aufweist. Dazu gehören reines Silizium, Siliziumcarbid $\mathrm{SiC}$ und Siliziumoxid $\mathrm{SiO}_{x}$.

Auch die Messungen des $\mathrm{C}$ 1s-Orbitals bestätigen das Vorliegen von $\mathrm{TiC}$ und $\mathrm{SiC}$ in der Probe. Das Ergebnis der XPS-Messung im Bereich von 280-288 eV zeigt Abbildung 6.16. Das Spektrum wurde in drei Peaks mit den Bindungsenergien 281,2 eV, 281,7 eV und $283,8 \mathrm{eV}$ entfaltet. Eindeutig identifiziert werden können dabei Peak $2 E_{B}=281,7 \mathrm{eV}$ 


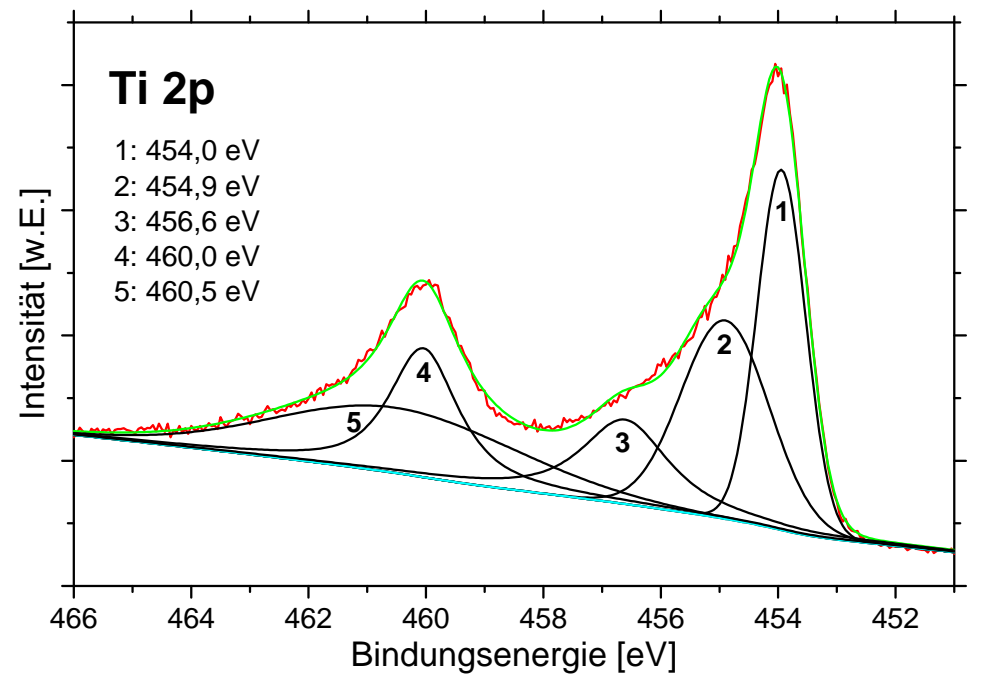

Abbildung 6.14: XPS-Messung des Ti 2p-Orbitals der bei Raumtemperatur ohne Ionenstrahl auf $\mathrm{Si}(100)$ deponierten Ti/Si/C-Schicht. Das Messspektrum (rot) wurde in fünf Peaks entfaltet (1-5, schwarz). Die grüne Linie zeigt die Summe dieser Peaks, die hellblaue Linie das subtrahierte Hintergrundsignal.

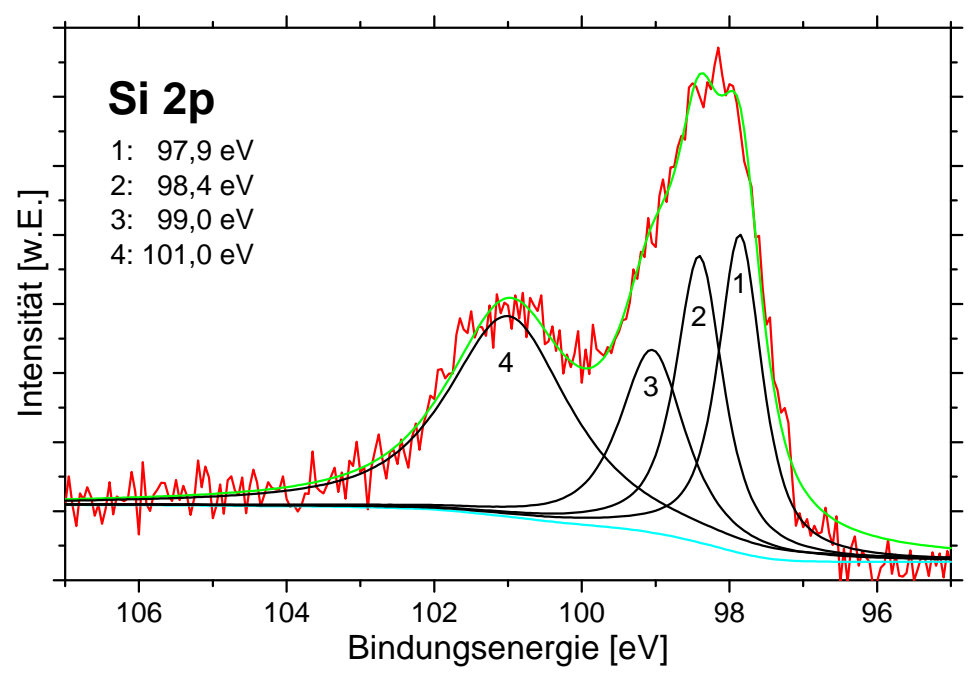

Abbildung 6.15: XPS-Messung des Si 2p-Orbitals der bei Raumtemperatur ohne Ionenstrahl auf $\mathrm{Si}(100)$ deponierten Ti/Si/C-Schicht. Das Messspektrum (rot) wurde in vier Peaks entfaltet (1-4, schwarz). Die grüne Linie zeigt die Summe dieser Peaks, die hellblaue Linie das subtrahierte Hintergrundsignal. 
als $\mathrm{C} 1 \mathrm{~s}$ in Titancarbid TiC und Peak $3 E_{B}=283,8 \mathrm{eV}$ als $\mathrm{C}$ 1s in Siliziumcarbid SiC. Für die Bindungsenergie von Peak $1 E_{B}=281,2 \mathrm{eV}$ findet sich wiederum kein Datenbankeintrag, der der chemischen Komposition der Probe entspricht. Allerdings gibt es zwei Spektrallinien für $\mathrm{SiC}$ und $\mathrm{TiC}$ bei $E_{B}=281,45 \mathrm{eV}$ bzw. $E_{B}=281,5 \mathrm{eV}$. Da die Intensität dieser Spektrallinie in der Messung relativ groß ist, und TiC die hauptsächlich in der Probe gebildete Phase ist, kann es sich auch hier wieder um eine Verzerrung der Linie durch die Schäden des Sputterionenstrahls handeln.

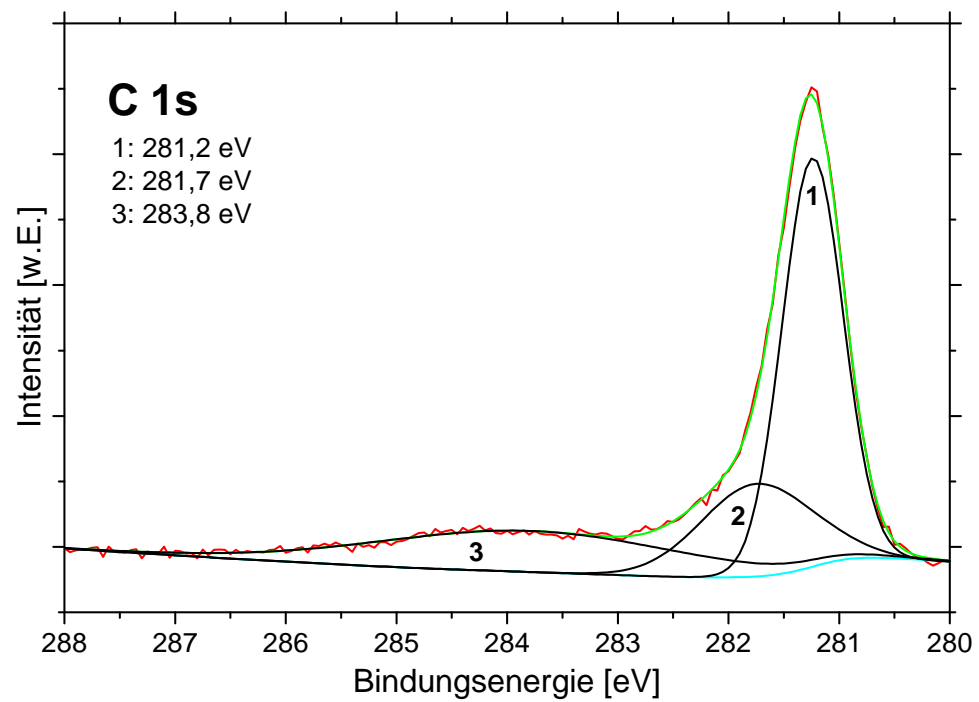

Abbildung 6.16: XPS-Messung des C 1s-Orbitals der bei Raumtemperatur ohne Ionenstrahl auf $\mathrm{Si}(100)$ deponierten Ti/Si/C-Schicht. Das Messspektrum (rot) wurde in drei Peaks entfaltet (1-3, schwarz). Die grüne Linie zeigt die Summe dieser Peaks, die hellblaue Linie das subtrahierte Hintergrundsignal.

Die Auswertung der XPS-Messungen liefert also wichtige zusätzliche Informationen über die chemischen Umgebungen in der Probe. Die Existenz der in den Röntgenbeugungsmessungen identifizierten Phase $\mathrm{TiC}$ wird bestätigt. Des Weiteren liegen auch geringe Mengen von Siliziumcarbid in der Probe vor. Der relativ hohe Sauerstoffgehalt der Schichten lässt sich durch die anteilige Oxidation von sowohl Titan zu $\mathrm{TiO}$ bzw. $\mathrm{TiO}_{x}$ als auch von Silizium zu $\mathrm{SiO}_{x}$ erklären.

\subsubsection{Elektrische Eigenschaften}

Eine wichtige Eigenschaft der MAX-Phasen ist ihre relativ hohe elektrische Leitfähigkeit. Aus diesem Grund wurden von den Proben, die auf MgO- und Si-Substraten hergestellt wurden, auch die Leitfähigkeiten gemessen. Da die Edelstahlsubstrate selbst elektrisch 
leitend sind, konnte auf ihnen die Leitfähigkeit der Schichten nicht bestimmt werden. Abbildung 6.17 zeigt die gemessenen Leitfähigkeiten der einzelnen Proben. Sie liegen alle in einem relativ engen Bereich von etwa $0,4-0,6 \cdot 10^{6}(\Omega \cdot \mathrm{m})^{-1}$. Vergleicht man die bei Raumtemperatur hergestellten Proben, sind die mit Ionenstrahl deponierten Schichten etwas leitfähiger als die ohne Ionenstrahl abgeschiedenen Proben. Ansonsten lassen sich keine eindeutigen Korrelationen aus dem Diagramm bestimmen. Die Leitfähigkeit von TiC liegt bei etwa $1,1 \cdot 10^{6}(\Omega \cdot \mathrm{m})^{-1}[16]$, die von reinem $\mathrm{Ti}_{3} \mathrm{SiC}_{2}$ beträgt $4,5 \cdot 10^{6}(\Omega \cdot \mathrm{m})^{-1}$ [15]. Emmerlich et al. bestimmten an dünnen $\mathrm{Ti}_{3} \mathrm{SiC}_{2}$-Schichten in Abhängigkeit vom Substrat Leitfähigkeiten von $4,0 \cdot 10^{6}(\Omega \cdot \mathrm{m})^{-1}$ auf $\mathrm{MgO}(111)$ bzw. $3,0 \cdot 10^{6}(\Omega \cdot \mathrm{m})^{-1}$ auf $\mathrm{Al}_{2} \mathrm{O}_{3}(0001)$ [38]. Alle hier gemessenen Proben liegen also unterhalb dieser Werte, und ihre Leitfähigkeiten sind eher mit denen von TiC vergleichbar, liegen aber dennoch unterhalb dessen Leitfähigkeit. In Anbetracht der Tatsache, dass die Schichten überwiegend aus einer TiC-Matrix bestehen, die durch verschiedene andere Oxid- und Carbidphasen gestört ist, war dieses Ergebnis zu erwarten.

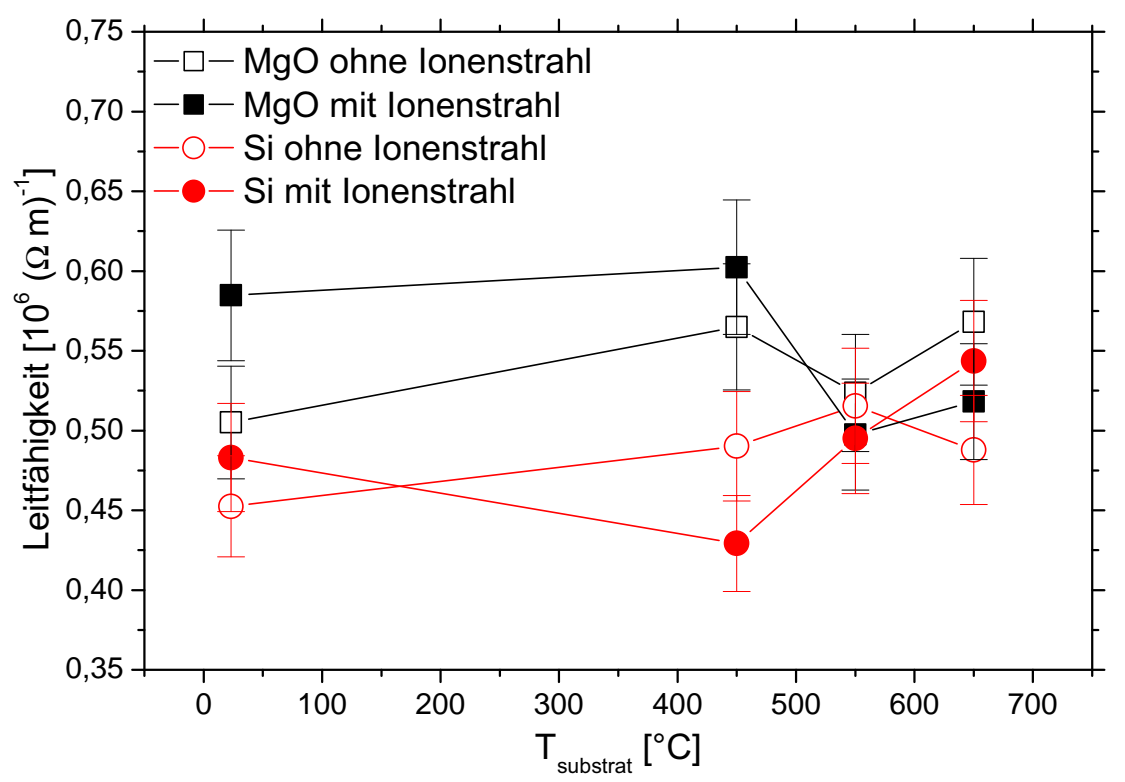

Abbildung 6.17: Leitfähigkeiten der auf $\mathrm{MgO}$ (schwarze Quadrate) und Si (rote Kreise) deponierten Ti/Si/C-Schichten. Offene Symbole: ohne Ionenstrahl, geschlossene Symbole: mit Ionenstrahl.

\subsubsection{Edelstahlsubstrat}

Da für eine technische Anwendung einkristalline Substrate wie $\mathrm{MgO}(100)$ und $\mathrm{Si}(100)$ kaum relevant sind, und außerdem bereits die Synthese dünner $\mathrm{Cr}_{2} \mathrm{AlC}-\mathrm{MAX}$-Schichten 
auf Edelstahl bei moderaten Temperaturen von $450{ }^{\circ} \mathrm{C}$ in der Literatur beschrieben wurde [42], wurden in dieser Arbeit auch Edelstrahlsubstrate für die Deposition verwendet.

\subsubsection{GDOES-Messungen}

Auch bei der Deposition auf Edelstahlsubstraten liegt die gewünschte stöchiometrische Zusammensetzung von $\mathrm{Ti}_{3} \mathrm{SiC}_{2}$ in den Schichten nicht vor. Die Konzentrationen $C_{i}$ von Titan, Silizium und Kohlenstoff sind etwas geringer als in den Schichten auf MgO beziehungsweise Si. Dafür ist der Sauerstoffgehalt noch ein wenig größer. Abbildung 6.18 zeigt die Übersicht der Konzentrationsanteile aller Elemente. Die Konzentrationen von Titan reichen von 40-51 at.-\%, die von Silizium betragen 6-13 at.-\% und die von Kohlenstoff 26-30 at.-\%. Der Sauerstoffanteil hingegen liegt in einem Bereich zwischen 7 und 20 at.- \% . Durch die Rauigkeit des Substrates und zusätzliche Diffusionseffekte enthalten die Schichten auch einen gewissen Anteil an Eisen aus dem Edelstahlsubstrat. Das Eisen ist dabei nicht in der gesamten Schicht verteilt, sondern reicht von der Grenzfläche zwischen Schicht und Substrat etwa $100 \mathrm{~nm}$ in den Film hinein. Insbesondere bei den Proben, die bei höherer Temperatur und mit Ionenstrahl hergestellt wurden, ist die Eisenkonzentration relativ hoch und beträgt in diesen Fällen etwa 9 at.-\%. Bei den anderen Proben liegt der Eisengehalt zwischen etwa 1 und 3 at.- $\%$.

Als wesentlicher Grund für die vergleichsweise hohe Sauerstoffkonzentration können das verwendete Substrat und sein Einfluss auf die kristallinen Eigenschaften der deponierten Schichten genannt werden. Der Stahl wurde nach dem Polieren ohne weitere Bearbeitung direkt beschichtet, und ist deswegen oberflächlich oxidiert. Des Weiteren ist die Oberfläche des Substrates im Vergleich zu den einkristallinen MgO- und Si-Substraten sehr viel rauer. Die Röntgenbeugungsmessungen in Abschnitt 6.1.4.2 zeigen, dass die kristalline Qualität der Filme bedeutend geringer ist, d.h. die Reflexe sind weniger stark ausgeprägt und fehlen - auch bei hohen Depositionstemperaturen - teilweise ganz. Dieses legt die Vermutung nahe, dass auf der schlecht definierten Edelstahloberfläche Titan und Kohlenstoff langsamer als auf den $\mathrm{MgO}$ - oder Si-Substraten kristalline Bereiche von TiC ausbilden. In dieser nur schwach kristallinen TiC-Matrix mit großen amorphen Bereichen ist die Diffusion von Sauerstoff in die Schicht dann stark begünstigt. Dadurch kann mehr Sauerstoff ins Innere der Schicht gelangen, und dieser wird dort durch die Metalle Titan und Silizium als Oxid gebunden. Die vermehrte Bildung der Oxide stört dann wiederum die weitere Kristallisation des $\mathrm{TiC}$, so dass sich beide Effekte während des Wachstums gegenseitig verstärken. Der Sauerstoffgehalt steigt folglich vom Substrat zur Filmoberfläche hin zunächst an, und fällt dann wieder etwas ab, da die Diffusion ins Schichtinnere eine gewisse Zeit benötigt. Abbildung 6.19 zeigt den Vergleich der bei $550{ }^{\circ} \mathrm{C}$ ohne Ionenstrahl deponierten Probe mit der bei $550{ }^{\circ} \mathrm{C}$ mit Ionenstrahl hergestellten Schicht. Die Messkurve für Eisen dient dabei als Indikator für das Edelstahlsubstrat. 


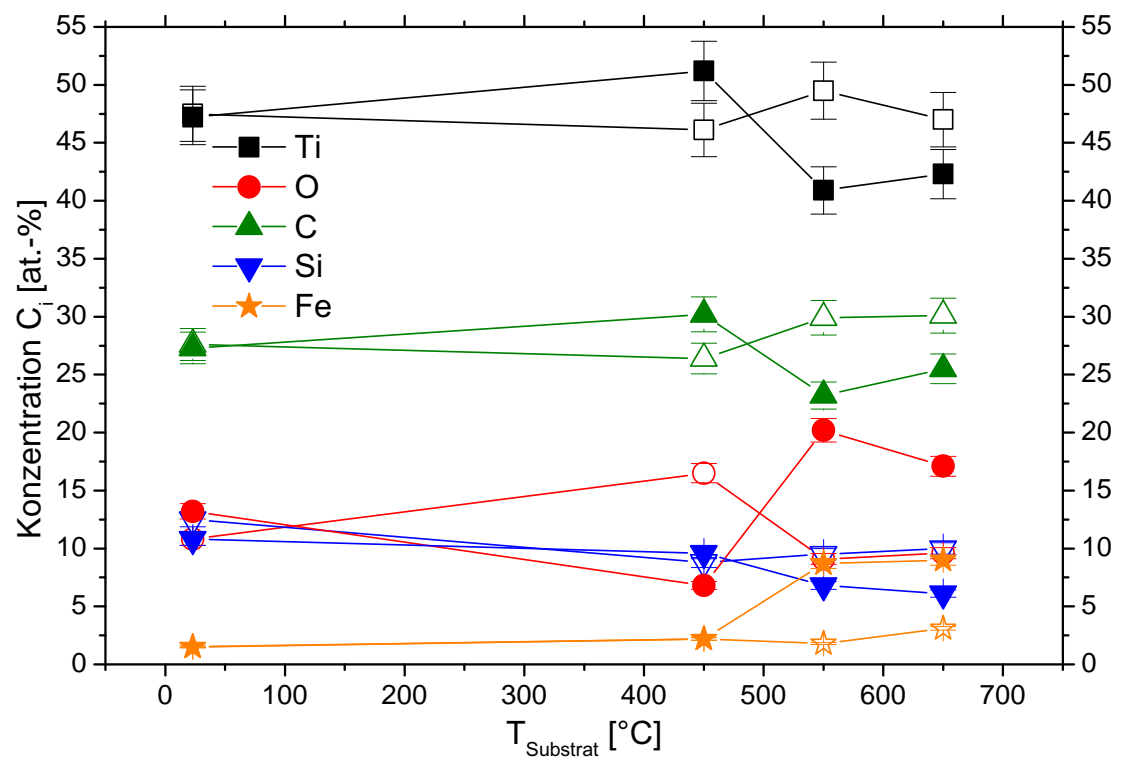

Abbildung 6.18: Mittlere Elementkonzentrationen $C_{i}$ der vom $\mathrm{Ti}_{3} \mathrm{SiC}_{2}$-Target auf Edelstahl deponierten Schichten in Abhängigkeit der Substrattemperatur während der Ablation. Offene Symbole: ohne Ionenstrahl, geschlossene Symbole: mit zugeschaltetem $200 \mathrm{~V}$ ArIonenstrahl. Die Linien dienen der besseren Orientierung.

Bei der Deposition mit Ionenstrahl zeigen sich die Diffusionseffekte noch extremer. Wie bereits in Abschnitt 6.1.1.1 angesprochen, wird durch die ungenügende Reinheit des verwendeten Argongases, sowie die hohe kinetische Energie des Ionenstrahls ohnehin mehr Sauerstoff in die Proben eingebracht. Durch die kinetische Energie der eingestrahlten Ionen werden Gitterschäden in der TiC-Matrix induziert, die die Sauerstoffdiffusion weiter begünstigen. Deswegen verschiebt sich das Maximum das Sauerstoffgehalts zur Substratoberfläche hin, und die lokale Sauerstoffkonzentration wird gleichzeitig noch größer. Zusätzlich kommt es an der Grenzschicht zwischen Substrat und Film zu erheblichen Diffusions- bzw. Vermischungserscheinungen. Die Eisenkurve steigt über einen großen Tiefenbereich (ca. $150 \mathrm{~nm}$ ) flach an, und die ablatierten Elemente Titan, Silizium und Kohlenstoff reichen weit in das Substrat hinein. In den Stahl sind 1,5-3,0 at.-\% Si einlegiert, deswegen fällt die Messkurve für Silizium nicht auf null ab.

Der hier diskutierte Diffusionseffekt mit der charakteristisch ansteigenden und abfallenden Sauerstoffkonzentration lässt sich auch auf die beiden anderen Substrate übertragen, ist dort aber weniger deutlich ausgesprägt (Abbildungen 6.3 und 6.10). Der Gesamtsauerstoffgehalt ist bei den Si-Proben höher, was auf eine allgemein weniger kristalline TiC-Matrix hindeutet. Bei der mit Ionenstrahl auf $\mathrm{MgO}$ hergestellten Probe (Abb. 6.3, rechtes Bild) ist das Sauerstoffmaximum relativ hoch und weniger breit. Dies deutet darauf hin, dass 

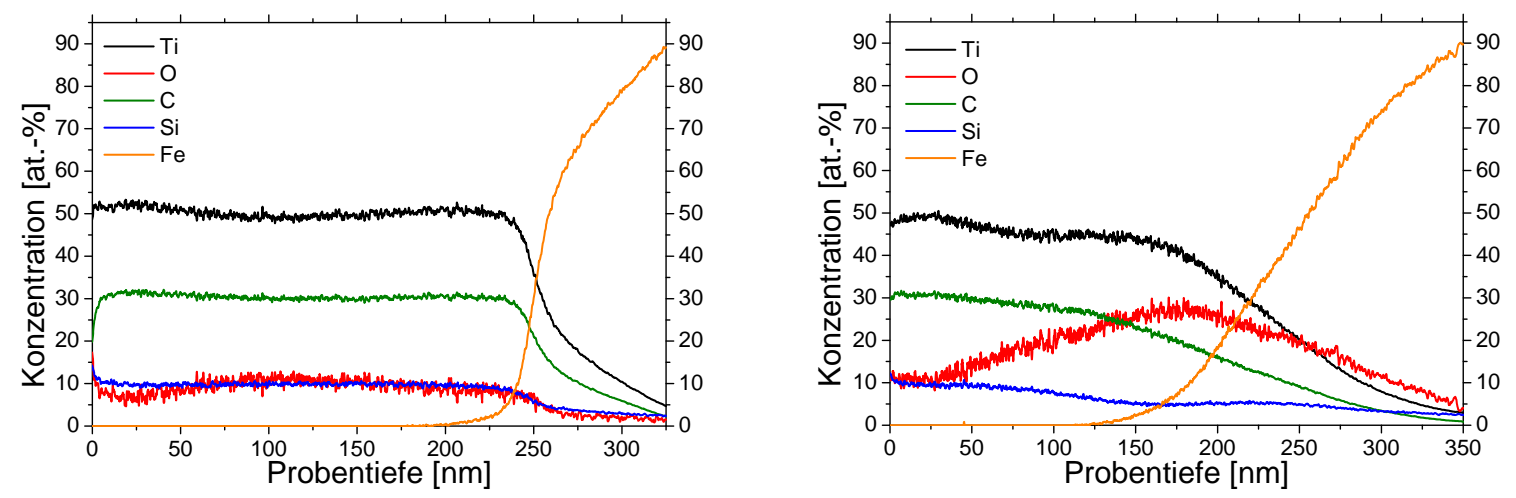

Abbildung 6.19: GDOES-Messungen zweier auf Edelstahl deponierter Ti/Si/C-Schichten. Links: $\mathrm{T}=550^{\circ} \mathrm{C}$, ohne Ionenstrahl, rechts: $\mathrm{T}=550^{\circ} \mathrm{C}$ mit $200 \mathrm{~V}$ Ar-Ionenstrahl. Deutlich zu erkennen ist die inhomogene Verteilung der Elemente bei Ionenbeschuss durch starke Diffusionseffekte.

beim Wachstum Titancarbid zunächst recht gut kristallisiert, bzw. die Schäden durch den Ionenstrahl schneller ausgeheilt werden. Erst später während der Deposition treten durch den Ionenstrahl stärkere Schäden in der Kristallstruktur auf, die eine verstärkte Sauerstoffdiffusion zur Folge haben.

Bei dieser Diskussion bleibt aber festzuhalten, dass bei gleichem Substrat die mit Ionenstrahl deponierten Schichten im Gegensatz zu den anderen Substraten trotz höherem Sauerstoffgehalt härter sind als die ohne Ionenstrahl hergestellten Proben. Hier scheint also der Einfluss des Ionenstrahls auf die Mikrostruktur in der Schicht und die daraus resultierende größere Härte (Hall-Petch-Beziehung) den Einfluss des höheren Sauerstoffgehaltes zu überwiegen.

\subsubsection{Röntgendiffraktometrie}

Auch auf den Edelstahlsubstraten ist kubisches TiC die einzige kristalline Phase. Wie bereits im vorhergehenden Abschnitt erwähnt, sind die Reflexe allerdings sehr viel weniger deutlich als auf den einkristallinen Substraten. Bei der Deposition ohne Ionenstrahl sind - abhängig von der Substrattemperatur - die TiC(200)-, TiC(111)- und TiC(220)Reflexe erkennbar (s. Abb. 6.20). Die weiter oben beschriebene Umkehrung der relativen Intensitäten zwischen dem $\mathrm{TiC}(200)$ - und dem $\mathrm{TiC}(111)$-Reflex wird ebenfalls beobachtet. Das Signal/Rausch-Verhältnis ist - bei vergleichbarer Schichtdicke - allerdings wesentlich schlechter als bei den auf $\mathrm{MgO}(100)$ bzw. Si(100) abgeschiedenen Proben, was auf einen deutlich geringeren Anteil von kristallinem TiC hindeutet. 


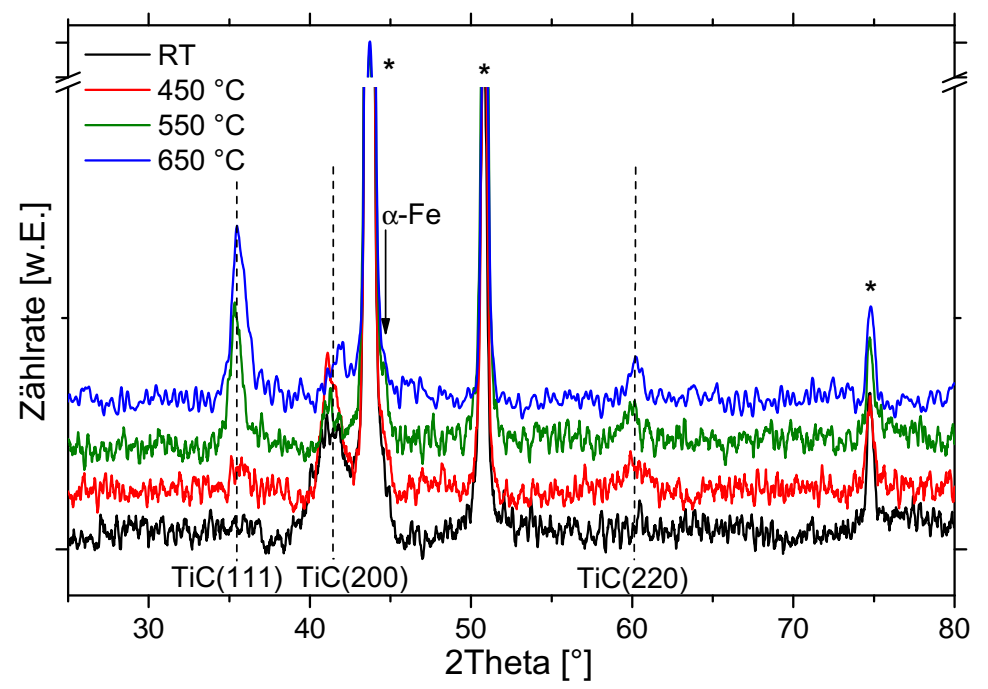

Abbildung 6.20: Röntgendiffraktogramme der Proben auf Edelstahl ohne Ionenstrahl. Indiziert sind die Reflexe für kubisches TiC. Die mit einem Stern markierten Reflexe stammen von $\gamma$-Eisen aus dem Edelstahlsubstrat. Außerdem teilweise zu erkennen ist der (110)-Reflex des $\alpha$-Eisen.

Noch schwächer sind die TiC-Reflexe bei den mit Ionenstrahl deponierten Schichten. Abbildung 6.21 zeigt, dass die Reflexe von $\mathrm{TiC}(200)$ und $\mathrm{TiC}(111)$ sehr klein sind, und die $\mathrm{TiC}(220)$-Reflexe nahezu verschwinden. Lediglich in der Messung der $650{ }^{\circ} \mathrm{C}$-Probe sind die Reflexe relativ gut zu erkennen, da diese Messung mit einer erheblich längeren Messdauer pro Schritt von 14 Sekunden durchgeführt wurde. Dennoch sind auch hier die Intensitäten der Reflexe sehr schwach. Erklärbar wird dies durch den Einfluss des Substrates, wie bereits in Abschnitt 6.1.4.1 erwähnt. Während die einkristallinen Substrate Si(100) und - aufgrund der großen kristallographischen Ähnlichkeit zu TiC - insbesondere $\mathrm{MgO}(100)$ relativ gute, definierte Oberflächen für ein kristallines Wachstum bieten, ist die Oberfläche der Edelstahlsubstrate extrem inhomogen. Obwohl die Oberflächen nach dem Schleifen poliert wurden, liegt die mittlere Rauigkeit $R_{a}$ immer noch bei etwa $15 \mathrm{~nm}$, also einem Bereich, der das Wachstum dünner Schichten schon erheblich beeinflusst. Des Weiteren ist die Oberfläche nicht nur topographisch, sondern auch kristallographisch nicht einheitlich. Neben der austenitischen $\gamma$-Eisenphase entstehen durch das Polieren an der Oberfläche auch Bereiche aus martensitischem $\alpha$-Eisen. Der (110)-Reflex von $\alpha$-Eisen bei $2 \Theta=44,6^{\circ}$ ist in den meisten Röntgendiffraktogrammen zu erkennen - entweder direkt als Peak oder als Schulter im Reflex des $\gamma$-Eisen. Durch diese Inhomogenitäten an der Substratoberfläche wird die Kristallisation der deponierten Atome erheblich gehindert. Weiterhin erschwerend dazu kommt die in den GDOES-Messungen (Abschnitt 6.1.4.1) gut erkennbare Diffusion von Eisen in die Schicht und von Titan, Silizium und Kohlen- 
stoff in das Edelstahlsubstrat.

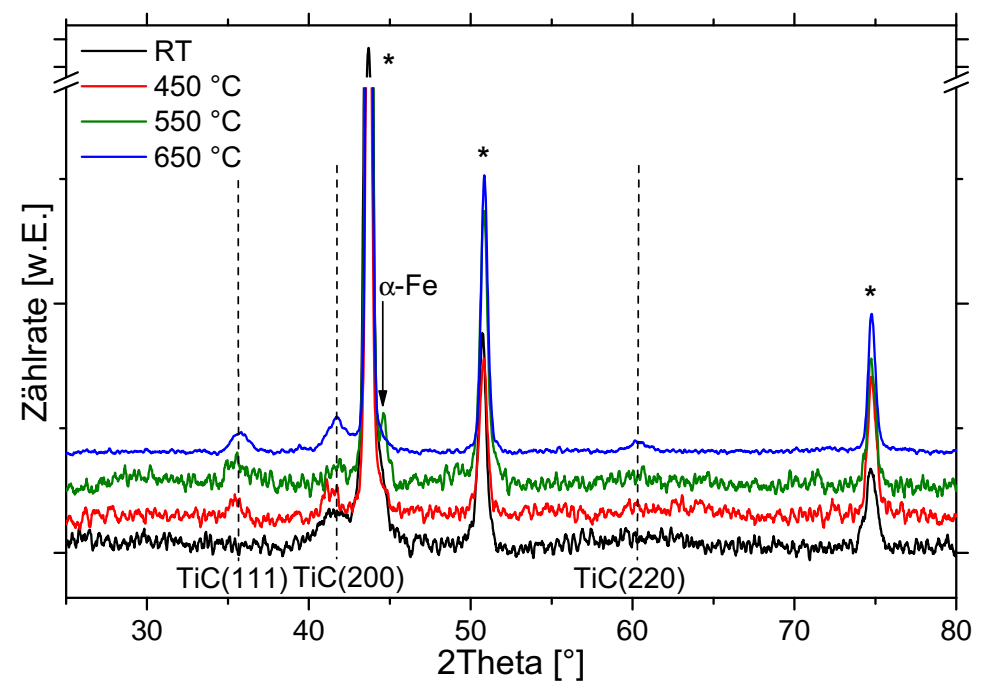

Abbildung 6.21: Röntgendiffraktogramme der Proben auf Edelstahl mit $200 \mathrm{~V}$ Ar-Ionenstrahl. Indiziert sind die Reflexe für kubisches TiC. Die mit einem Stern markierten Reflexe stammen von $\gamma$-Eisen aus dem Edelstahlsubstrat. Außerdem teilweise zu erkennen ist der (110)-Reflex $\operatorname{des} \alpha$-Eisen.

\subsubsection{Härtemessungen}

Entsprechend der obigen Diskussionen zu Kristallinität, Sauerstoffgehalt und Diffusionserscheinungen sind die Schichthärten auf den Edelstahlsubstraten sehr klein. Sie reichen von etwa 3,7 bis 6,3 GPa. Entgegen dem Verhalten auf $\mathrm{MgO}$ und Si nimmt in diesem Fall die Härte der Schicht nicht mit der Depositionstemperatur zu, sondern ab, obwohl auch hier mit zunehmender Substrattemperatur die Röntgenbeugungsreflexe stärker wurden. Eine Erklärung dafür wäre - durch die Diffusionen begünstigt - die Ausbildung kristalliner TiC-Domänen in einer röntgenamorphen Matrix, während im Fall von $\mathrm{MgO}$ und Si eher eine kristalline TiC-Matrix mit weitestgehend amorphen Einschlüssen von Titan, Silizium und deren Oxiden vorliegt. Die einzelnen Messungen der Härtewerte streuen mehr als bei den Messungen auf den einkristallinen Substraten, was durch die angezeigten größeren Fehler erkennbar ist. Die Edelstahl-Proben sind also makroskopisch inhomogener, was ebenfalls auf einzelne härtere TiC-Bereiche hindeutet. Gleichzeitig senkt auch eine inhomogene Zusammensetzung, die angesichts der Diffusionserscheinungen bei höheren Temperaturen zunimmt, die Gesamthärte der Schichten. 


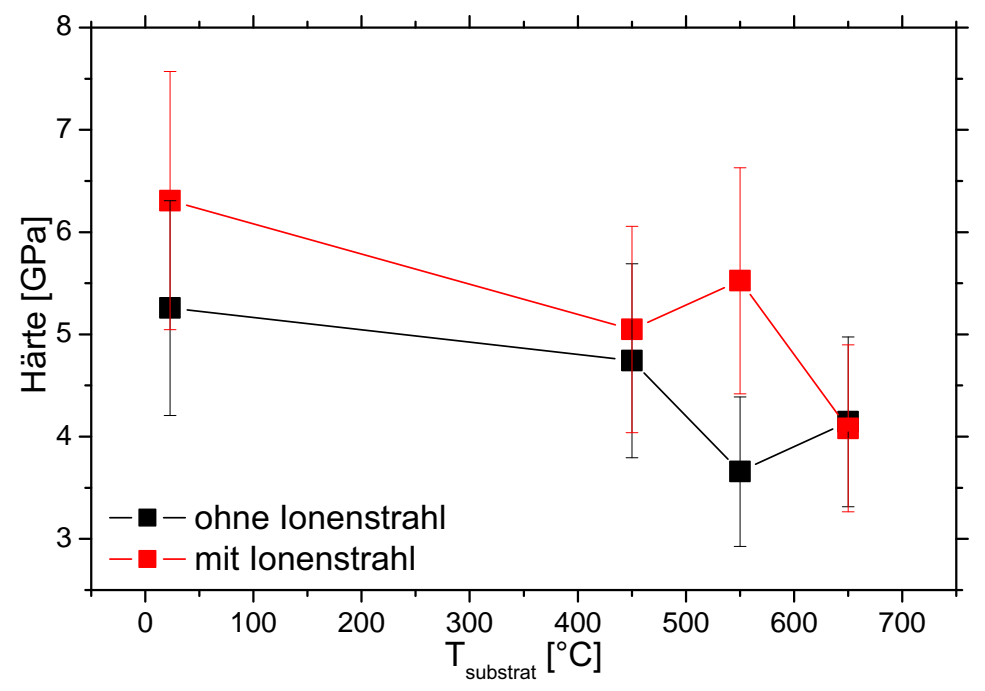

Abbildung 6.22: Schichthärten der auf Edelstahl deponierten Proben als Funktion der Substrattemperatur. Schwarz: ohne Ionenstrahl, rot: mit Ionenstrahl. Die Linien dienen der besseren Orientierung.

\subsubsection{Zusammenfassung $-\mathrm{Ti}_{3} \mathrm{SiC}_{2}$-System}

Es ist gelungen, mit Hilfe der ionenstrahlunterstützten Pulslaserdeposition von einem $\mathrm{Ti}_{3} \mathrm{SiC}_{2}$-MAX-Phasentarget homogene und rissfreie Schichten auf verschiedenen Substraten herzustellen. Dabei lag in keiner der Proben die gewünschte stöchiometrische $\mathrm{Ti}_{3} \mathrm{SiC}_{2}{ }^{-}$ Zusammensetzung vor. Es kommt zu Verlusten vor allem des Siliziums beim Übertrag vom Target in die Schicht. Dies lässt sich vor allem durch ein selektives Absputtern von Silizium aus der Schicht durch die hohe kinetische Energie der ablatierten Teilchen erklären. Titan und Kohlenstoff dagegen bilden relativ schnell das Carbid $\mathrm{TiC}$ und werden dadurch besser im Film gebunden. Außerdem kam es zu einer signifikanten Inkorporation von Sauerstoff durch Diffusion und Oxidation, die sich in erster Linie durch ungenügende Vakuumbedingungen während der Ablation erklären lässt.

Die einzige identifizierbare kristallographische Phase ist in allen Fällen kubisches Titancarbid TiC. Es bildete sich in den Schichten ein Gefüge aus amorphen Bereichen und kleinen Kristalliten. Die Kristallite bestehen dabei überwiegend aus TiC. In den TEMAufnahmen ließen sich aber auch kleine kristalline TiO-Strukturen identifizieren. Wie die Elektronenbeugungsmessungen gezeigt haben, wächst zumindest bei hohen Substrattemperaturen und ohne Ionenstrahl das $\mathrm{TiC}$ auf $\mathrm{MgO}(100)$ mit einer leicht ausgeprägten Vorzugsrichtung auf. Dabei ist das Gitter des $\mathrm{TiC}$ um etwa $20^{\circ}$ gegenüber der Gitteroberfläche des Substrates verdreht. 
Die überwiegende Bildung von $\mathrm{TiC}$ war insofern zu erwarten, als dass $\mathrm{TiC}$ die zur MAXPhase $\mathrm{Ti}_{3} \mathrm{SiC}_{2}$ gehörende binäre MX-Verbindung darstellt, und die Umwandlung beider Phasen ineinander theoretisch nur den geordneten Einbau des A-Elements - in diesem Fall Silizium - voraussetzt, der bisher allerdings nur bei sehr hohen Substrattemperaturen beobachtet wurde. Unterhalb dieser Bildungstemperatur kam es wiederum zum Entstehen einer kristallinen TiC-Matrix, in die das amorphe Silizium eingebettet ist. Die XPS-Messungen zeigen außerdem die Existenz von elementarem Titan und Silizium, sowie Siliziumcarbid und der Oxide $\mathrm{TiO}, \mathrm{TiO}_{x}$ und $\mathrm{SiO}_{x}$ an. Dabei ist allerdings zu beachten, dass es durch das Absputtern der Oberfläche vor den XPS-Messungen zu erheblichen lokalen Störungen in der Probe kommen kann, die die Emissionslinien - insbesondere von Kohlenstoff - zum Teil verzerren.

Sowohl die Substrattemperatur während der Deposition, als auch das gewählte Substrat selbst und die Benutzung des Ionenstrahls haben Einfluss auf die Eigenschaften der dünnen Schichten. Die einkristallinen Substrate mit definierter Oberfläche ermöglichen ein besseres Kristallwachstum von TiC, das durch höheren Substrattemperaturen noch begünstigt wird. Die Schichten werden dadurch ebenfalls härter. Die auf MgO(100) gewachsenen Filme zeigen dabei eine größere Härte als die Schichten auf $\mathrm{Si}(100)$. Insgesamt liegen die Schichthärten mit Werten von 7-13 GPa bei etwa der Hälfte von reinen TiCSchichten, für die Härten von 15 bis über $30 \mathrm{GPa}$ gemessen wurden. Im Vergleich zu MAX-Phasen (4-6 GPa) sind sie jedoch härter. Bei höheren Temperaturen kommt es allerdings zu einer vermehrten Aufnahme von Sauerstoff in die Proben, die sich auf die Schichthärte auswirkt. So sind die Schichten mit einem höheren Sauerstoffgehalt weicher. Vermutlich beeinflussen sich Sauerstoffdiffusion und Kristallisation des TiC dabei gegenseitig. Die Bildung der Oxide behindert das Kristallwachstum des Titancarbid und an den entsprechend zahlreicheren amorphen Bereichen kann wiederum der Sauerstoff besser in die Schicht hinein diffundieren. Diese Sauerstoffdiffusion wird durch die Anwendung des Ar-Ionenstrahls und der dadurch entstehenden Schäden im Film noch begünstigt, wie auch die GDOES-Sauerstoffprofile zeigen. Interessanterweise sind jedoch die mit Ionenstrahl hergestellten Proben härter als die ohne Ionenstrahl deponierten Filme, obwohl sie mehr Sauerstoff enthalten. Es gibt also noch einen weiteren Einfluss der Ionenbestrahlung, der Einfluss auf die Schichthärte hat, und unabhängig von der Sauerstoffkonzentration ist. Die Mikrostruktur der Schichten - insbesondere die mittlere Größe der Kristallite - wird durch den Ionenbeschuss verändert. Entsprechend der Hall-Petch-Beziehung kommt es durch die Verkleinerung der kristallinen Bereiche zunächst zu einer größeren Schichthärte, da die Bewegung von Versetzungen unterdrückt wird. Unterschreiten die Kristallite jedoch eine kritische Größe $d_{c}$ werden die Schichten wieder weicher, da nun das Gleiten von Korngrenzen zum Abbau mechanischen Stresses auftritt. Wie die TEM-Aufnahmen zeigen, liegt die Größe der kristallinen Bereiche in den Proben im Bereich dieser kritischen Größe $d_{c}$. In den Proben kommt es also zu einer Konkurrenz zwischen Effekten, die die 
Schichthärte erhöhen und solchen, die die Schichthärte verringern. Die Benutzung des Ionenstrahls verschiebt in den meisten Fällen dieses Gleichgewicht zugunsten einer höheren Schichthärte.

Bei der Betrachtung der Schichthärten ist noch ein weiterer Einfluss zu beachten: der des Substrats. Obwohl die Eindringtiefe des Prüfkörpers bei keiner Messung $30 \%$ der Filmdicke überschritten hat, die Schichten also nicht durchstoßen wurden, lässt sich ein Einfluss der Härte des darunterliegenden Substrates auf die gemessene Gesamthärte nicht ausschließen, da die einwirkenden Kräfte durchaus längere Reichweiten haben als die Eindringtiefe der Prüfspitze. Die verwendeten Substrate haben aber stark verschiedene Härten: $H_{\mathrm{MgO}} \approx 10,0 \mathrm{GPa}, H_{\mathrm{Si}} \approx 6,5 \mathrm{GPa}$ und $H_{\text {Stahl }} \approx 3,0 \mathrm{GPa}$. Die für die unterschiedlichen Substrate gemessenen Schichthärten sind dadurch möglicherweise ebenfalls mit beeinflusst.

Die elektrische Leitfähigkeiten $\sigma$ der Filme auf $\mathrm{MgO}$ und Si betragen $0,4-0,6 \cdot 10^{6}(\Omega \cdot \mathrm{m})^{-1}$. Sie liegen damit eine Größenordnung unterhalb der Leitfähigkeit von $\mathrm{Ti}_{3} \mathrm{SiC}_{2}$-Vollmaterial $\left(\sigma=4,5 \cdot 10^{6}(\Omega \cdot \mathrm{m})^{-1}\right)$, bzw. $\mathrm{Ti}_{3} \mathrm{SiC}_{2}$-Schichten $\left(\sigma=3,0-4,0 \cdot 10^{6}(\Omega \cdot \mathrm{m})^{-1}\right)$ und auch noch unterhalb der Leitfähigkeit von reinem $\operatorname{TiC}\left(\sigma=1,1 \cdot 10^{6}(\Omega \cdot \mathrm{m})^{-1}\right)$. Die Ergebnisse sind also am ehesten mit denen von $\mathrm{TiC}$ zu vergleichen. Allerdings ist die TiC-Matrix in den Proben auch erheblich durch Oxide gestört, und folglich die Leitfähigkeit herabgesetzt.

Weniger gut definiert sind die auf Edelstahl deponierten Schichten. Aufgrund der topographischen (Oberflächenrauigkeit) und chemischen (Phasengemisch aus austenitischem und martensitischem Stahl) Inhomogenität des Substrates ist die Kristallisation von Titancarbid bereits von Anfang an stark gehindert. Dadurch werden Diffusionsprozesse begünstigt, die sowohl die Diffusion von Sauerstoff in die Schicht als auch den Austausch von Elementen an der Grenzfläche zwischen Schicht und Substrat betreffen. Die Konsequenzen daraus sind ein sehr hoher Sauerstoffgehalt, geringere - in GI-Geometrie gemessene - Kristallinität, eine deutlich Inhomogenität der Proben und folglich niedrigere Schichthärten (3,5-6,5 GPa). Für eine technische Anwendung sind diese Ergebnisse der Deposition auf Edelstahl jedoch nicht uninteressant. Wie in Abschnitt 2.1 dargestellt, finden Komposite aus nano-kristalline $\mathrm{TiC}$ mit amorphen Siliziumeinschlüssen mittlerweile Verwendung als Schutzschicht auf elektrischen Kontakten. In Hinblick auf diese Art der Anwendung muss aber noch das Problem der hohen Sauerstoffaufnahme gelöst werden.

Im Fall der Deposition von $\mathrm{Ti}_{3} \mathrm{SiC}_{2}$ auf $\mathrm{Si}(100)$ bei $650{ }^{\circ} \mathrm{C}$ mit Ionenstrahl riss der Film bereits während der Deposition. Nimmt man an, dass sich durch die hohe Substrattemperatur und den zusätzlichen Einfluss des Ionenstrahls zunächst relativ viel $\mathrm{TiC}$ an der Substratoberfläche gebildet hat, kann man das Reißen der Schicht durch die hohe Gitterfehlanpassung erklären. Sowohl TiC als auch Silizium bilden eine kubische Struktur. Die Gitterparameter $a$ sind in beiden Verbindungen stark verschieden. In TiC beträgt die Kantenlänge der Elementarzellen $a=0,432 \mathrm{~nm}$, in Silizium hingegen $a=0,543 \mathrm{~nm}$. Dies 
entspricht einer Gitterfehlanpassung von etwa $20 \%$. Beim Wachstum von $\mathrm{TiC}$ auf $\mathrm{MgO}$ ( $a=0,421 \mathrm{~nm}$ ) beträgt die Fehlanpassung weniger als $3 \%$. Es tritt also erheblich weniger Stress bei der Deposition auf, und die Schicht reißt bei gleichen Depositionsparametern nicht. 


\section{2 $\mathrm{Cr}_{2}$ AIC-Target}

Die REM-Aufnahme des Querschnittes durch eine bei $550{ }^{\circ} \mathrm{C}$ deponierte $\mathrm{Cr}_{2} \mathrm{AlC}$-Probe ist in Abbildung 6.23 gezeigt. Zu erkennen ist ein etwa $650 \mathrm{~nm}$ dicker kompakter Film. Im Gegensatz zur Ablation vom $\mathrm{Ti}_{3} \mathrm{SiC}_{2}$-Target kam es bei den $\mathrm{Cr}_{2} \mathrm{AlC}$-Proben nicht zum spannungsbedingten Reißen der Beschichtung auf dem Substrat. Alle Proben bestanden aus homogenen und rissfreien Filmen.

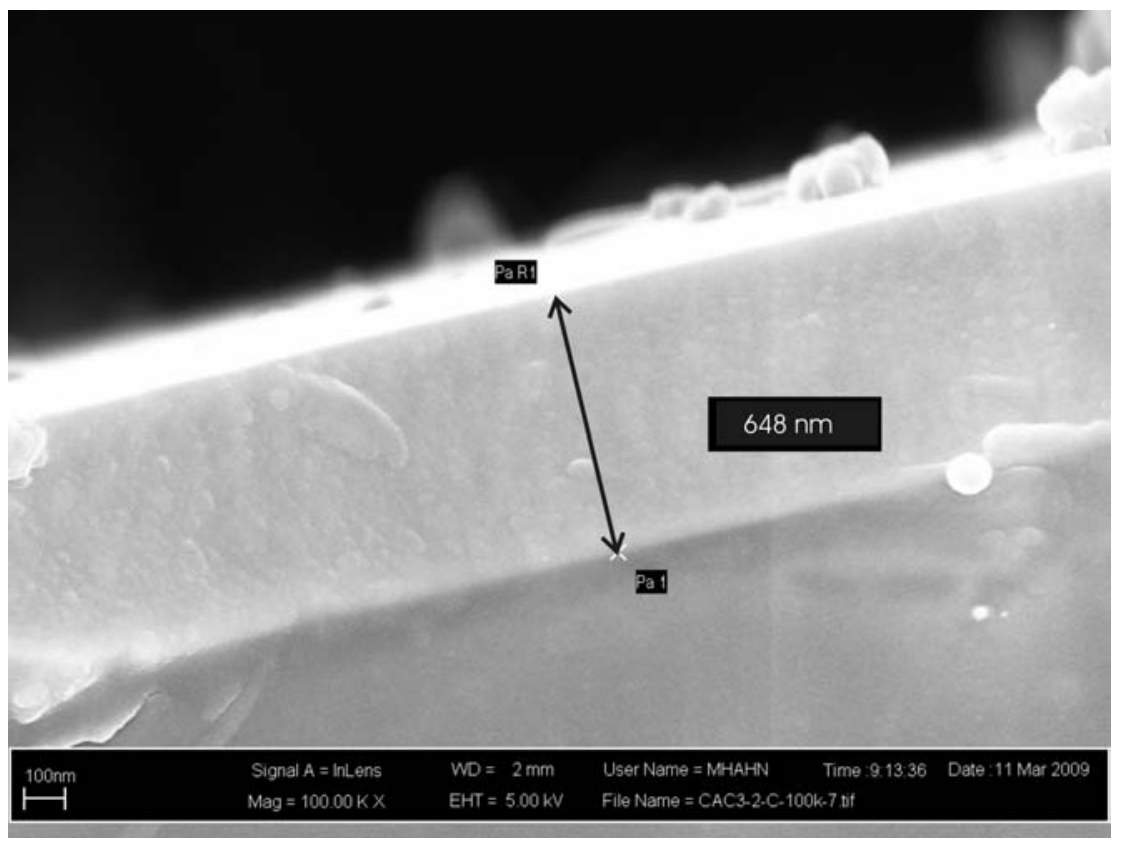

Abbildung 6.23: REM-Aufnahme des Querschnitts durch eine bei $550{ }^{\circ} \mathrm{C}$ auf $\mathrm{MgO}(100)$ deponierte $\mathrm{Cr}_{2} \mathrm{AlC}$-Schicht. Die kugeligen Verunreinigungen an der Oberfläche stammen vom für die Mikroskopie aufgedampften Kohlenstoff.

\subsubsection{MgO(100)-Substrat}

\subsubsection{GDOES-Messungen}

Die mit der Glimmentladungsspektroskopie gemessene Zusammensetzung aller Proben unterscheidet sich deutlich von der stöchiometrischen Zusammensetzung des $\mathrm{Cr}_{2} \mathrm{AlC}$-Targets (50 at.- \% Cr, 25 at.-\% Al und 25 at.-\% C). Die Filme enthalten in aller Regel deutlich mehr Chrom, weniger Kohlenstoff und noch weniger Aluminium als das Targetmaterial. In Abhängigkeit der Depositionsparameter liegen der Chromgehalt zwischen 58 und 71 at.- $\%$, der Aluminiumgehalt zwischen 9 und 26 at.- $\%$ und der Kohlenstoffgehalt zwischen 14 und 20 at.-\%. Die Sauerstoffkonzentration in den Proben ist sehr niedrig und schwankt in einem Bereich von 0,3 bis 1,8 at.-\%. Die mittleren Elementkonzentrationen 
$C_{i}$ aller auf $\mathrm{MgO}$ deponierten Schichten sind als Funktion der Substrattemperatur in Abbildung 6.24 dargestellt. Genau wie bei der Ablation des $\mathrm{Ti}_{3} \mathrm{SiC}_{2}$-Targets kommt es also zu einem selektiven Verlust bestimmter Elemente durch das Wiederabsputtern aufgrund der hohen kinetischen Energien der auftreffenden Teilchen. Der Kohlenstoffgehalt der Schichten nimmt bei steigender Substrattemperatur leicht zu, was auf eine zunehmende Chromcarbidbildung in den Schichten hindeutet. Eine nennenswerte Oxidation der Schichten - wie bei den $\mathrm{Ti}_{3} \mathrm{SiC}_{2}$-Proben - findet dagegen nicht statt. Offensichtlich sind die Elemente Titan und Silizium bei der PLD bedeutend sauerstoffaffiner als die Elemente Chrom und Aluminium.

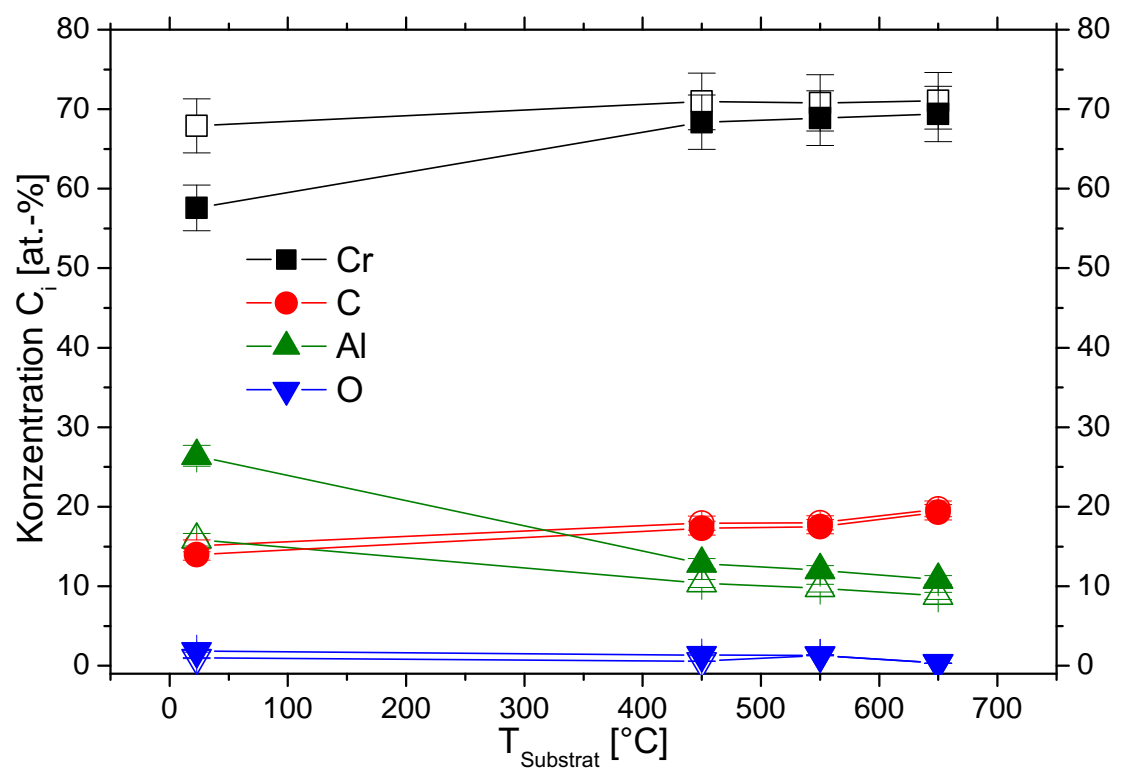

Abbildung 6.24: Mittlere Elementkonzentrationen $C_{i}$ der vom $\mathrm{Cr}_{2} \mathrm{AlC}$-Target auf $\mathrm{MgO}$ deponierten Schichten in Abhängigkeit der Substrattemperatur während der Ablation. Offene Symbole: ohne Ionenstrahl, geschlossene Symbole: mit zugeschaltetem $200 \mathrm{~V}$ ArIonenstrahl. Die Linien dienen der besseren Orientierung.

Es ist zu erkennen, dass - außer in einem Fall - die Benutzung des Argon-Ionenstrahls relativ wenig Einfluss auf die Zusammensetzung der Proben hat. Lediglich der Chromanteil sinkt in geringem Maße bei Zuschaltung des Ionenstrahl zugunsten des Aluminiumanteils. Besonders drastisch ist dieser Effekt jedoch bei der Deposition bei Raumtemperatur. Hier beträgt die Aluminiumkonzentration 26 at.- \%, während sie sonst im Bereich von 10-15 at.-\% liegt. Der Chromanteil schwankt dagegen in allen anderen Fällen in einem engen Bereich zwischen 68 und 71 at.- $\%$ und fällt bei der Raumtemperaturdeposition mit Ionenstrahl auf 58 at.-\% ab. Die Betrachtung des Tiefenprofils der entsprechenden Probe zeigt dabei, dass das Aluminium nicht homogen in der Schicht verteilt ist, son- 
dern die Aluminiumkonzentration zur Grenzfläche zwischen Substrat und Film hin ansteigt (Abbildung 6.25, rechtes Bild). Zum Vergleich ist in der Abbildung ebenfalls die bei Raumtemperatur ohne Ionenstrahl deponierte Probe gezeigt. Auch hier kommt es zu einem Konzentrationsanstieg des Aluminium in einem Bereich von der Substratoberfläche bis etwa $50 \mathrm{~nm}$ (ca. $10 \%$ der Schichtdicke) in die Schicht hinein. Die maximale lokale Konzentration des Aluminium liegt in beiden Fällen oberhalb der 25 at.-\%, die im Target vorliegen. Offensichtlich ist Aluminium relativ mobil im Vergleich zu den anderen Elementen, kann deswegen sehr gut diffundieren und reichert sich zunächst an der Substratoberfläche an. Bei der Deposition ohne Ionenstrahl fällt der Al-Gehalt dann relativ schnell in Richtung Schichtoberfläche zu einem konstanten Wert von etwa 15 at.-\% ab, der dem tatsächlichen Übertrag von Aluminium aus dem Target in die Schicht entspricht. Das Aluminium wird also nicht stöchiometrisch übertragen, sondern es kommt zu einem Gleichgewicht zwischen den Aluminiumdeposition und Absputtern aus der Schicht durch die kinetische Energie der auftreffenden ablatierten Partikel. Wird die Schicht dagegen mit Ionenstrahl deponiert, kommt es zu einer weiteren Erhöhung der Mobilität der AlAtome. Die Aluminiumanreicherung über 25 at.-\% erfolgt über einen größeren Bereich in der Schicht, und das Gleichgewicht zwischen Deposition und Absputtern wird in Richtung einer erhöhten Deposition verschoben. Auffällig bei der Anreicherung des Aluminiums ist das spiegelbildliche Verhalten der Konzentrationsprofile von Chrom und Aluminium. Im beinahe selben Maße, in dem sich die Aluminiumkonzentration erhöht, wird der Chromanteil in der Schicht abgesenkt. Dieses Verhalten lässt sich durch einen Austausch von Chrom- und Aluminiumatomen in der Schicht interpretieren. Tatsächlich löst sich Aluminium in Chrom bis zu einem Anteil von 33 at.- $\%$ Al. Es kommt also zur Formation dieser festen Lösung von Aluminium in Chrom in den Bereichen der erhöhten Al-Konzentration. Bei der Zusammensetzung 33 at.- $\%$ Al/67 at.-\% Cr bildet sich dann die Phase $\mathrm{Cr}_{2} \mathrm{Al}$ [59]. Die Ergebnisse der Röntgenbeugung und der TEM-Mikroskopie im folgenden Abschnitt 6.2.1.2 zeigen in einigen Proben die Existenz dieser Phase, was gleichzeitig das Vorhandensein der festen Lösung impliziert.

Dieser Anreicherungseffekt tritt in dieser Deutlichkeit nur bei den Depositionen bei Raumtemperatur auf. Werden die Schichten bei höheren Temperaturen hergestellt, kommt es zwar auch, wie in Abbildung 6.26 für $\mathrm{T}=650{ }^{\circ} \mathrm{C}$ gezeigt, zu einer Erhöhung der Aluminiumkonzentration in Richtung der Substratoberfläche. Diese ist jedoch nicht so stark ausgeprägt. Hier beträgt der Anstieg des Al-Gehaltes nur etwa 5 at.- $\%$ bei den Depositionen ohne Ionenstrahl und etwa 9 at.-\% bei Benutzung des Ionenstrahls. Durch den Temperatureinfluss scheinen sich also die Mobilitäten der einzelnen Elemente anzunähern, so dass es insgesamt zu einer gleichmäßigeren Verteilung innerhalb der Schichten kommt, die dabei aber immer noch die Anreicherung des Aluminiums in Richtung der Substratoberfläche aufweisen. Gleichzeitig findet bei hohen Temperaturen auch die Carbidbildung schneller statt, die eine Konkurrenzreaktion zur Bildung der festen Lösung darstellt. Das 

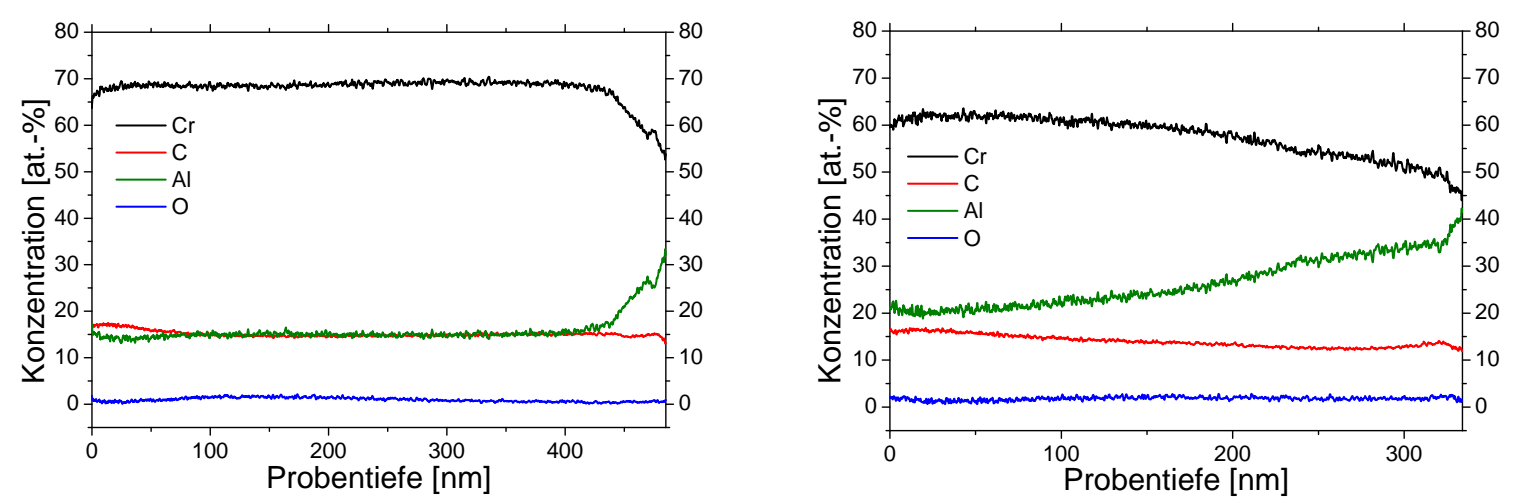

Abbildung 6.25: GDOES-Messungen zweier bei Raumtemperatur auf $\mathrm{MgO}(100)$ deponierter Cr/Al/C-Schichten. Links: $\mathrm{T}=\mathrm{RT}$, ohne Ionenstrahl, rechts: $\mathrm{T}=\mathrm{RT}$, mit $200 \mathrm{~V}$ ArIonenstrahl. Deutlich zu erkennen ist das inhomogene Aluminium-Konzentrationsprofil mit der lokalen Anreicherung in Richtung der Substratoberfläche.

Aluminium kann sich also nicht mehr gut im Chrom lösen. Beide $650{ }^{\circ} \mathrm{C}$-Proben weisen eine Aluminiumkonzentration von etwa 10 at.- $\%$ an der Schichtoberfläche auf, die wiederum dem gleichgewichtsbedingten Übertrag von Al aus dem Target in die Schicht entspricht. Der Einfluss des Ionenstrahls zeigt sich aber auch hier in dem eben erwähnten größeren Konzentrationsunterschied des Aluminiums zwischen Schicht- und Substratoberfläche und in einer Vergrößerung des Bereiches, in dem diese Konzentrationsänderung stattfindet. Ohne Ionenstrahl sind dies etwa $40 \mathrm{~nm}$ (ca. $16 \%$ der Schichtdicke), mit Ionenstrahl etwa $80 \mathrm{~nm}$ (ca. $34 \%$ der Schichtdicke) von der Substratoberfläche in die Schicht.
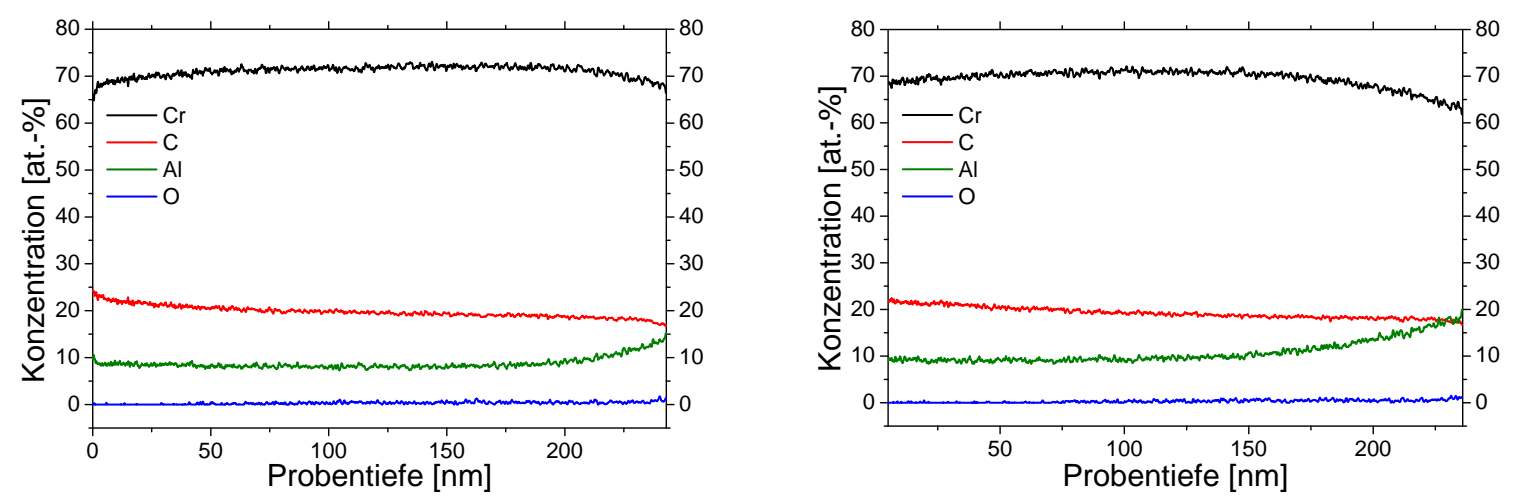

Abbildung 6.26: GDOES-Messungen zweier bei $650{ }^{\circ} \mathrm{C}$ auf $\mathrm{MgO}(100)$ deponierter $\mathrm{Cr} / \mathrm{Al} / \mathrm{C}$ Schichten. Links: $\mathrm{T}=650^{\circ} \mathrm{C}$, ohne Ionenstrahl, rechts: $\mathrm{T}=650^{\circ} \mathrm{C}$, mit $200 \mathrm{~V}$ Ar-Ionenstrahl. Im Vergleich zu den Raumtemperaturproben ist die lokale Anreicherung von Aluminium deutlich weniger ausgeprägt. 
Um genauere Informationen über die Anreicherung des Aluminiums zu erhalten, wurde zum Vergleich eine weitere Schicht bei Raumtemperatur deponiert, bei der der $200 \mathrm{~V}$ Ionenstrahl erst nach der Hälfte der Depositionszeit zugeschaltet wurde. Aus experimentellen Gründen lag der Hintergrunddruck dabei von Anfang an bei $4 \cdot 10^{-2} \mathrm{~Pa}$. Das bedeutet, dass die knietische Energie der ablatierten Partikel durch den höheren Druck geringer ist als im Hochvakuum. Bei dieser Probe tritt keine lokale Konzentrationsänderung im Aluminiumprofil auf (Abbildung nicht gezeigt). Die Anreicherung von Aluminium tritt folglich nur auf, wenn Teilchen von Anfang an mit höherer kinetischer Energie - entweder die Atome aus dem Target im Hochvakuum oder die Argonionen aus dem Ionenstrahl auf das Substrat treffen. Die Abscheidung direkt auf die Substratoberfläche spielt dabei also eine wichtige Rolle. Ist dies nicht der Fall bildet sich von Anfang an eine Matrix aus Chromcarbiden, in denen es nicht zu einer Konzentrationserhöhung des Aluminiums kommt.

\subsubsection{Röntgendiffraktometrie und TEM-Messungen}

Während die bei Raumtemperatur hergestellten Schichten in beiden Fällen röntgenamorph sind, entstehen bei höheren Temperaturen in Abhängigkeit von der Substrattemperatur zwei verschiedene kristalline Phasen in den Proben. Diese sind das Chromcarbid $\mathrm{Cr}_{7} \mathrm{C}_{3}$ (ICSD 36-1482), sowie in zwei Fällen die intermetallische Phase $\mathrm{Cr}_{2} \mathrm{Al}$ (ICSD 29-0016). Die Abbildungen 6.27 und 6.28 zeigen die Diffraktogramme aller Proben in Abhängigkeit der Substrattemperatur während der Deposition. Gemessen wurde mit $5^{\circ}$ streifendem Einfall (GI) bei einer Schrittweite von $0,02^{\circ}$ und einer Messdauer von 1 s pro Schritt. Bei der Deposition ohne Ionenstrahl, ist - wie bereits erwähnt - die Raumtemperaturprobe röntgenamorph. Es ist lediglich ein sehr breiter und flacher Beugungsreflex im Bereich von $2 \Theta=43^{\circ} \mathrm{zu}$ erkennen. Bei $450^{\circ} \mathrm{C}$ erscheinen Reflexe von $\mathrm{Cr}_{7} \mathrm{C}_{3}$ und $\mathrm{Cr}_{2} \mathrm{Al}$. Bei steigender Temperatur verschwindet die Phase $\mathrm{Cr}_{2} \mathrm{Al}$ wieder. Die Beugungsreflexe werden mit zunehmender Temperatur schärfer, was auf eine Vergrößerung der einzelnen Kristallite hindeutet. Wie die TEM-Aufnahme in Abbildung 6.30 zeigt, bestehen die Schichten auch hier wieder aus einem Gefüge von amorphen und kristallinen Bereichen.

Ähnliches gilt für die mit Ionenstrahl hergestellten Schichten. Auch hier ist die Raumtemperaturprobe röntgenamorph, und es kommt bei $450{ }^{\circ} \mathrm{C}$ zur Bildung von $\mathrm{Cr}_{7} \mathrm{C}_{3}$ und $\mathrm{Cr}_{2} \mathrm{Al}$. Die intermetallische Phase ist bei höheren Temperaturen wiederum nicht erkennbar. Als kristalline Phase existiert dann lediglich das Chromcarbid $\mathrm{Cr}_{7} \mathrm{C}_{3}$.

Die Zusammensetzung der auf $\mathrm{MgO}$ deponierten kristallinen Proben liegt bei etwa 6872 at.\% Cr, 18-20 at.-\% C und 8-12 at.-\% Al. Dieser Bereich ist in Abbildung 6.29 mit einem roten Kreuz gekennzeichnet. Im thermischen Gleichgewicht sollten demnach in der Probe das Carbid $\mathrm{Cr}_{7} \mathrm{C}_{3}$, eine feste Lösung von Aluminium in Chrom - hier als (Cr) 


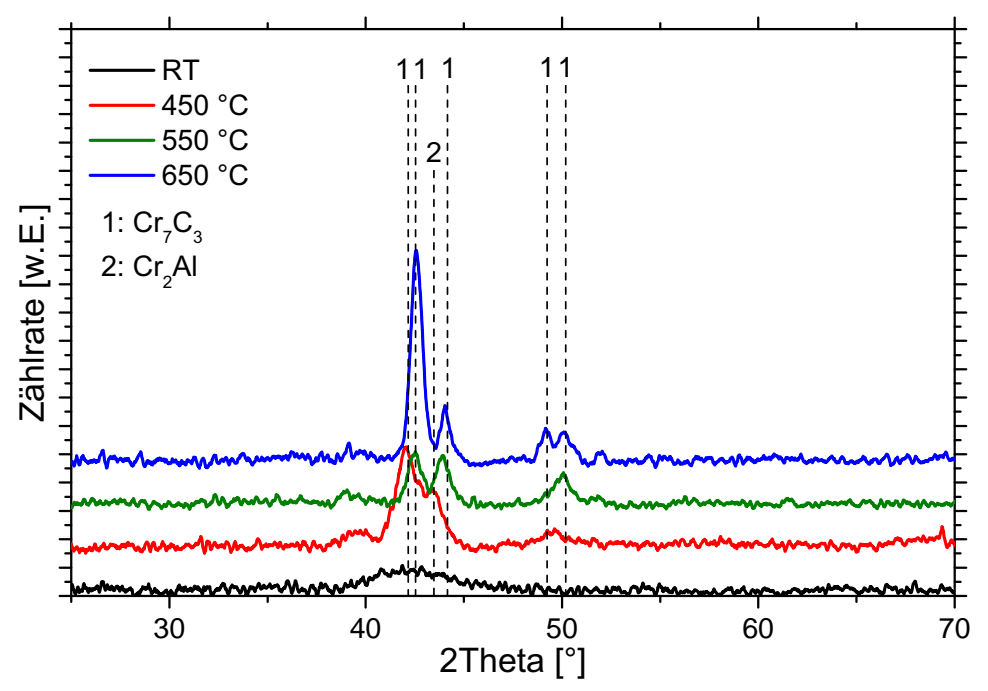

Abbildung 6.27: Röntgendiffraktogramme der $\mathrm{Cr}_{2} \mathrm{AlC}-$ Proben auf $\mathrm{MgO}$ ohne Ionenstrahl. In Abhängigkeit von der Substrattemperatur bilden sich die Phasen $\mathrm{Cr}_{7} \mathrm{C}_{3}$ (1) und $\mathrm{Cr}_{2} \mathrm{Al}$ (2). Bei Raumtemperatur ist die Schicht röntgenamorph.

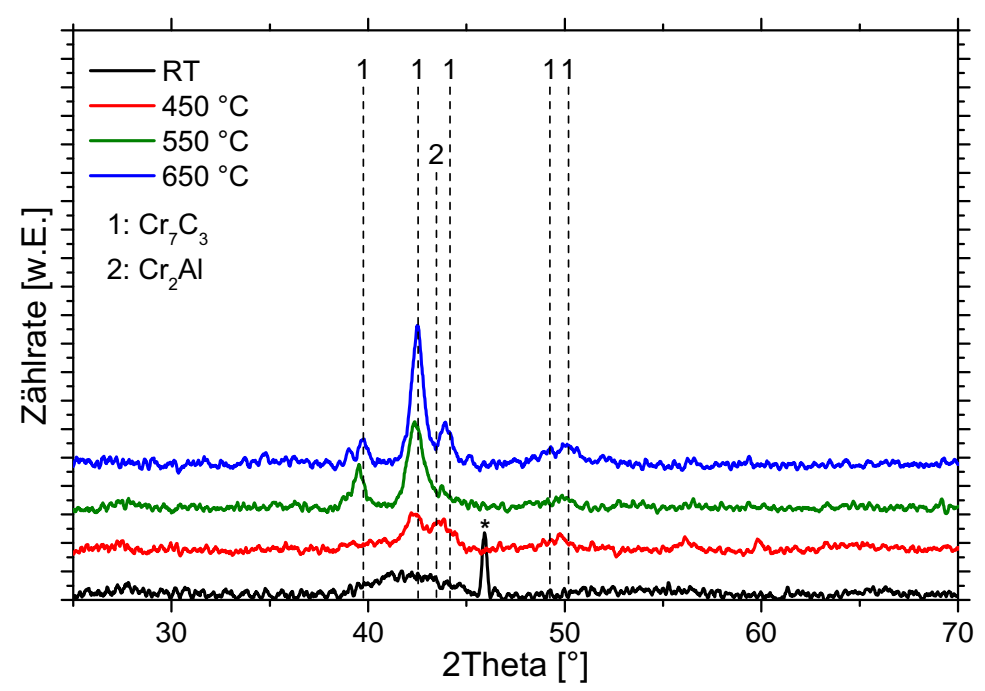

Abbildung 6.28: Röntgendiffraktogramme der $\mathrm{Cr}_{2} \mathrm{AlC}-$ Proben auf $\mathrm{MgO}$ mit Ionenstrahl. Abhängig von der Substrattemperatur bilden sich die $\mathrm{Phasen} \mathrm{Cr}_{7} \mathrm{C}_{3}$ (1) und $\mathrm{Cr}_{2} \mathrm{Al}$ (2). Bei Raumtemperatur ist die Schicht röntgenamorph. Der scharfe mit einem Stern markierte Reflex ist ein Messartefakt, das vom Substrat stammt.

gekennzeichnet - und, je nach konkreter Zusammensetzung, sehr geringe Mengen der MAX-Phase $\mathrm{Cr}_{2} \mathrm{AlC}$ (hier als $\tau$ bezeichnet) vorliegen. Dünne PLD-Schichten stellen aber 
eher Ungleichgewichtszustände dar, so dass die im Phasendiagramm angegebenen Verbindungen nicht notwendigerweise als kristalline Phase im Röntgenbeugungsdiagramm erscheinen. Die hochgradig geordnete Struktur der MAX-Phasen zum Beispiel entsteht in dünnen Schichten nur unter besonderen Bedingungen, was Temperatur und Oberflächenbeschaffenheit des Substrates angeht (vgl. [38, 55]). Betrachtet man die Ergebnisse aus GDOES und XRD zusammen, lässt sich für die Zusammensetzung der Proben eine mehr oder weniger kristalline Matrix aus $\mathrm{Cr}_{7} \mathrm{C}_{3}$ mit einer röntgenamorphen festen Lösung aus Aluminium in Chrom in Zwischenräumen und an Korngrenzen annehmen.

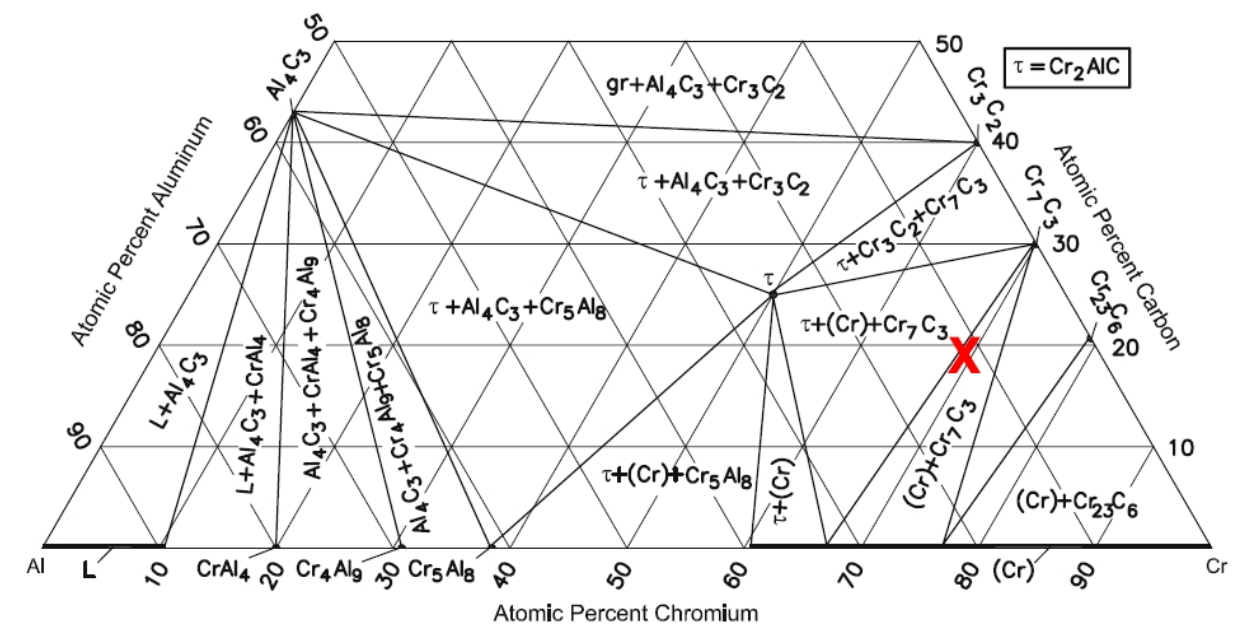

Abbildung 6.29: Ternäres Cr-Al-C-Phasendiagramm. Der Zusammensetzungsbereich der kristallinen $\mathrm{MgO}-$ Proben ist durch das rote Kreuz gekennzeichnet.

Eine Ausnahme bilden dabei die beiden bei einer Substrattemperatur von $450{ }^{\circ} \mathrm{C}$ hergestellten Schichten. Nur hier sind - sowohl bei der Benutzung des Ionenstrahls als auch bei der Abscheidung ohne Ionenstrahl - Reflexe der intermetallischen Phase $\mathrm{Cr}_{2} \mathrm{Al} z u$ sehen. Im binären Phasendiagramm von $\mathrm{Cr}$ und $\mathrm{Al}$ ist $\mathrm{Cr}_{2} \mathrm{Al}$ die Phase, die die feste (Cr,Al)-Lösung in Richtung höhere Aluminiumkonzentration begrenzt [59]. Die Bildung von $\mathrm{Cr}_{2} \mathrm{Al}$ aus der festen Lösung ist also unter bestimmten Umständen auch in dünnen Schichten durchaus möglich. Wie im vorherigen Abschnitt bereits erwähnt, hängt das Auftreten dieser Phase mit der lokalen Konzentrationserhöhung des Aluminiums an der Grenzfläche zwischen Substrat und Schicht zusammen. $\mathrm{Cr}_{2} \mathrm{Al}$ kristallisiert in der tetragonalen Raumgruppe $P 4 / \mathrm{mmm}$. Die quadratische Grundfläche der Elementarzelle von $\mathrm{Cr}_{2} \mathrm{Al}$ hat eine Kantenlänge von $a=0,300 \mathrm{~nm}$. Die Länge der Diagonale durch die Grundfläche beträgt folglich $d=0,300 \mathrm{~nm} \cdot \sqrt{2}=0,424 \mathrm{~nm}$. Das kubische MgO-Substrat hat den Gitterparameter $a=0,421 \mathrm{~nm}$. $\mathrm{Cr}_{2} \mathrm{Al}$ kann also auf $\mathrm{MgO}$ - um $45^{\circ}$ verdreht mit einer Gitterfehlanpassung von lediglich 0,7 \% epitaktisch aufwachsen. Dadurch wird zu Beginn des Filmwachstums die Bildung von $\mathrm{Cr}_{2} \mathrm{Al}$ aus der festen Lösung von $\mathrm{Al}$ in Cr begünstigt. In der festen Lösung und der auftretenden intermetallischen Phase ist das Aluminium stärker gebunden, als später in der Matrix der sich bildenden Chromcarbide. 
Dadurch kommt es zu der beobachteten Konzentrationserhöhung des Aluminiums in der Substrat-Film-Grenzschicht. Bereits nach kurzer Wachstumsdauer (wenige Nanometer) wird die $\mathrm{Cr}_{2} \mathrm{Al}$-Phase polykristallin - und damit in der GI-Röntgenbeugung erkennbar. Im weiteren Verlauf der Deposition bilden sich dann bevorzugt die Chromcarbide. Das Aluminium wird nicht mehr so stark im Film gebunden, und die Al-Konzentration fällt auf den geringeren Gleichgewichtswert aus Deposition und Absputtern ab. Bei Raumtemperatur ist der Al-Gehalt in der Grenzschicht zwar am höchsten, allerdings kommt es nicht zur Bildung einer kristallinen Phase; die Schichten sind röntgenamorph. Eine Temperatur von $450{ }^{\circ} \mathrm{C}$ ist ausreichend, um das soeben beschriebene Wachstumsszenario zu ermöglichen. Bei höheren Temperaturen verhindert dann die erhöhte Beweglichkeit auch der anderen Atome durch Diffusion die Ausbildung einer polykristallinen $\mathrm{Cr}_{2} \mathrm{Al}$-Schicht, weswegen keine Beugungsreflexe mehr beobachtet werden können.

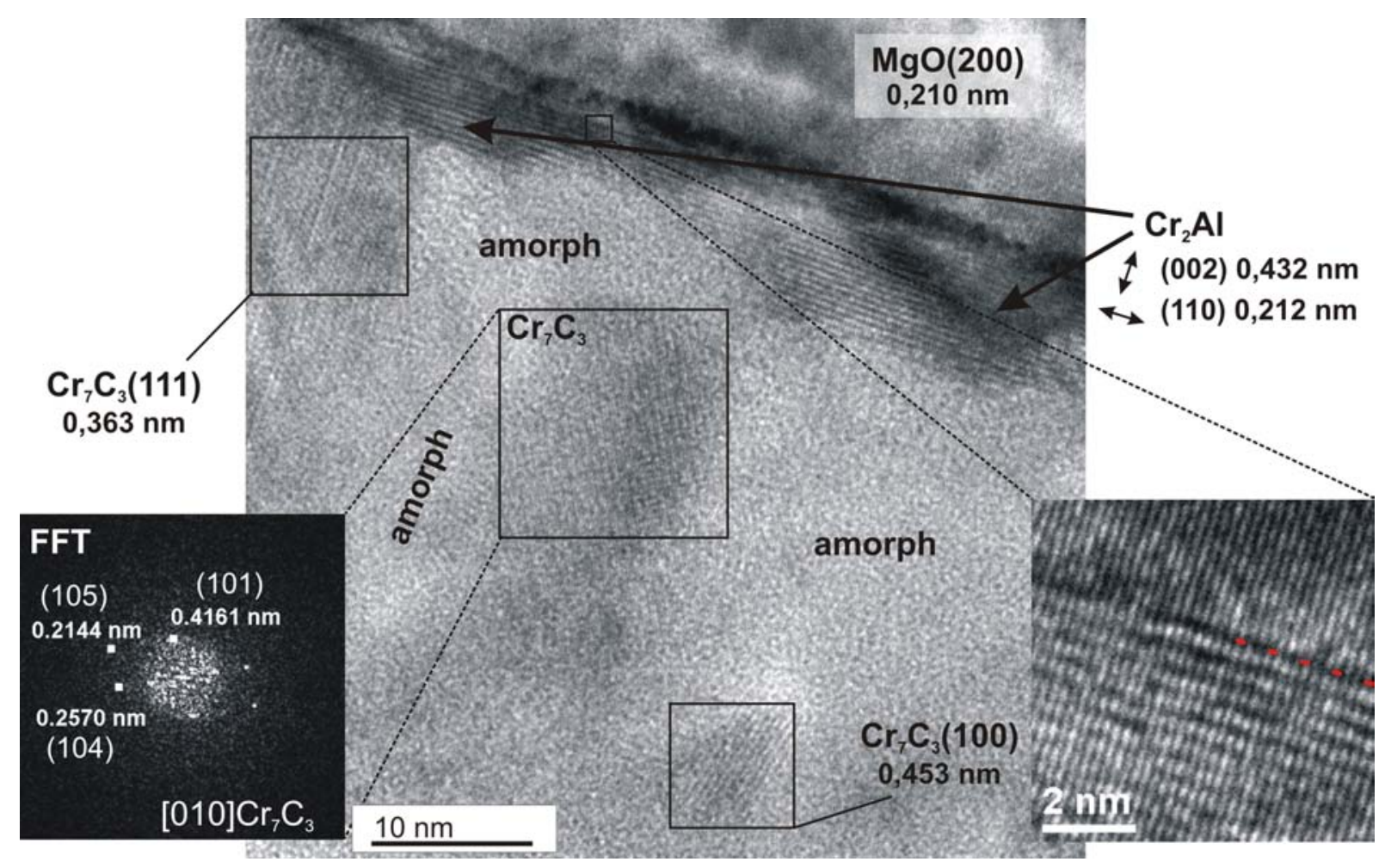

Abbildung 6.30: HR-TEM-Aufnahme des Übergangsbereiches zwischen Substrat und Schicht der bei $450{ }^{\circ} \mathrm{C}$ ohne Ionenstrahl deponierten Probe. Das Gefüge der Probe aus kristallinen und amorphen Bereichen, sowie das epitaktische Wachstum von $\mathrm{Cr}_{2} \mathrm{Al}$ auf dem Substrat sind deutlich zu erkennen. Unten links eingeklinkt: schnelle Fourier-Transformation (FFT) eines kristallinen $\mathrm{Cr}_{7} \mathrm{C}_{3}$-Bereiches, unten rechts eingeklinkt: Ausschnittsvergößerung des Übergangs vom Substrat in die Schicht. Die rote Strichlinie deutet den Verlauf der Substratoberfläche an.

Ein epitaktisches Wachstum an der Grenzfläche zwischen Substrat und Schicht lässt sich tatsächlich auch mit der hochauflösenden Transmissionselektronenmikroskopie (HR- 
TEM) beobachten. Abbildung 6.30 zeigt eine solche HR-TEM-Aufnahme der bei $450{ }^{\circ} \mathrm{C}$ ohne Ionenstrahl hergestellten Probe (großes Bild). Direkt an der Grenzfläche zwischen $\mathrm{MgO}-$ Substrat und Schicht sind in diesem Bild dabei hochgradig geordnete Bereiche zu erkennen, die der Phase $\mathrm{Cr}_{2} \mathrm{Al}$ zuzuordnen sind. Die Atomabstände parallel zur Substratoberfläche betragen dabei $0,212 \mathrm{~nm}$, was dem (110)-Netzebenenabstand von $\mathrm{Cr}_{2} \mathrm{Al}$ $\left(d_{I C S D}=0,2122 \mathrm{~nm}\right)$ entspricht. Senkrecht zur Substratoberfläche beträgt der mittlere Atomabstand 0,432 $\mathrm{nm}$ und stimmt damit mit dem (002)-Ebenenabstand in $\mathrm{Cr}_{2} \mathrm{Al}$ $\left(d_{I C S D}=0,4318 \mathrm{~nm}\right)$ überein. Das kleine, unten rechts eingeklinkte Bild zeigt eine Ausschnittsvergrößerung des Übergangs vom Substrat zur Schicht. Dabei deutet die rote Strichlinie den Verlauf der Substratoberfläche an. Gut zu sehen ist die epitaktische Struktur des $\mathrm{Cr}_{2} \mathrm{Al}$ mit leichten Gitterverzerrungen aufgrund von Fehlanpassungen und Energieminimierungen.

Außerdem in der Aufnahme zu erkennen sind einzelne kristalline Bereiche von $\mathrm{Cr}_{7} \mathrm{C}_{3}$. Im größten dieser Bereiche in der Mitte des Bildes wurde eine schnelle Fourier-Transformation (FFT) durchgeführt, deren Ergebnis in der kleinen, links unten eingeklinkten Abbildung dargestellt wurde. Die markierten diskreten Punkte können dabei den Netzebenenabständen (101), (104) und (105) in $\mathrm{Cr}_{7} \mathrm{C}_{3}$ zugeordnet werden, so dass sich als Zonenachse $\mathrm{Cr}_{7} \mathrm{C}_{3}$ [010] ergibt. In den anderen markierten $\mathrm{Cr}_{7} \mathrm{C}_{3}$-Bereichen wurden lediglich der Abstand der Atomreihen ausgemessen und die Miller-Indizes der entsprechenden Netzebenenscharen $\mathrm{Cr}_{7} \mathrm{C}_{3}$ (100) bzw. $\mathrm{Cr}_{7} \mathrm{C}_{3}$ (111) angegeben.

\subsubsection{Härtemessungen}

Abhängig von den Depositionsparametern weisen die Proben Schichthärten von etwa 9 bis $11 \mathrm{GPa}$ auf. Dabei nimmt die Härte mit steigender Substrattemperatur zu, was sich anhand der in der Röntgenbeugung zu sehenden zunehmenden Kristallinität der Proben erklären lässt. Die mit Ionenstrahl hergestellten Schichten sind unwesentlich härter als die ohne Ionenstrahl deponierten. Unter Betrachtung der XRD-Messungen kann man von etwas kleineren Kristalliten in den mit Ionenstrahl abgeschiedenen Schichten ausgehen. Dabei liegt die Größe der Kristallite aber noch überhalb der kritischen Größe $d_{c}$ der HallPetch-Beziehung, so dass die Proben eine leicht erhöhte Schichthärte aufweisen. Da die $\mathrm{Cr} / \mathrm{Al} / \mathrm{C}-\mathrm{Schichten}$ im Gegensatz zu den Ti/Si/C-Proben kaum Sauerstoff enthalten, tritt keine Verringerung der Schichthärte durch den Einfluss von Sauerstoff auf.

Der Vergleich der hier gemessenen Werte mit anderen Literaturwerten gestaltet sich relativ schwierig, da es nur wenig Veröffentlichungen zu reinen Chromcarbidschichten gibt, und noch seltener deren Schichthärten gemessen wurden. Hirota et al. geben für die Härten der Vollmaterialien von $\mathrm{Cr}_{3} \mathrm{C}_{2}, \mathrm{Cr}_{7} \mathrm{C}_{3}$ und $\mathrm{Cr}_{23} \mathrm{C}_{2}$ folgende Werte an [151]: $H\left(\mathrm{Cr}_{3} \mathrm{C}_{2}\right)=$ 15-19 GPa, $H\left(\mathrm{Cr}_{7} \mathrm{C}_{3}\right)=16-17 \mathrm{GPa}$ und $H\left(\mathrm{Cr}_{23} \mathrm{C}_{6}\right)=14-15 \mathrm{GPa}$. 


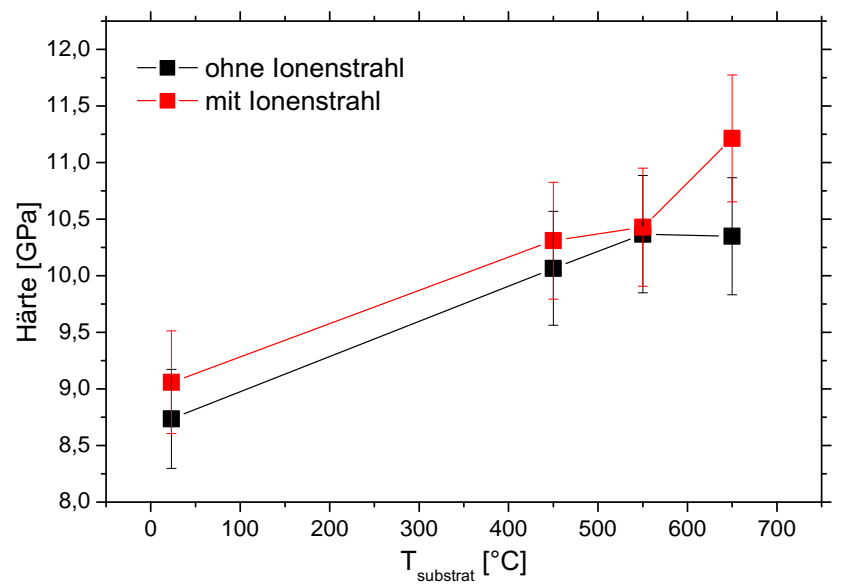

Abbildung 6.31: Schichthärten der auf $\mathrm{MgO}$ deponierten $\mathrm{Cr}_{2} \mathrm{AlC}-$ Proben (schwarz: ohne Ionenstrahl, rot: mit Ionenstrahl).

Die maximalen Werte von 10-11 GPa liegen also etwa 30-35 \% unterhalb der für Vollmaterialien gemessenen Härten. Schichthärten von Chromcarbiden wurden bisher nur für Cr:C-Verhältnisse bestimmt, die sich von denen in dieser Arbeit unterscheiden. An Schichten der Zusammensetzung $\mathrm{Cr}: \mathrm{C} \approx 3: 2$, in denen sich hauptsächlich das Chromcarbid $\mathrm{Cr}_{3} \mathrm{C}_{2}$ bildete, bestimmten Maréchal et al. Schichthärten von 7-12 GPa [152]. Chrom/Kohlenstoff-Schichten mit noch höherem Kohlenstoffanteil ( $\mathrm{Cr}: \mathrm{C} \approx 1: 2$ ), die allerdings kaum noch kristalline Eigenschaften hatten, zeigten Härten von 8-20 GPa [153]. Bei der Untersuchung von dünnen Schichten aus phasenreinem $\mathrm{Cr}_{2} \mathrm{AlC}$ wurden vergleichbare

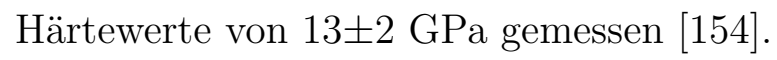

\subsection{2 $\mathrm{SiO}_{2}$-Substrat}

\subsubsection{GDOES-Messungen}

In Abbildung 6.32 sind die mit GDOES gemessenen mittleren Elementkonzentrationen $C_{i}$ aller auf $\mathrm{SiO}_{2}$ deponierten Schichten dargestellt. Wiederum entsprechen diese - aufgrund des selektiven Absputterns - nicht der stöchiometrischen Zusammensetzung des $\mathrm{Cr}_{2} \mathrm{AlC}-$ Targets. In den Filmen findet sich sehr viel mehr Chrom (60-71 at.-\%), aber weniger Kohlenstoff (15-21 at.-\%) und ebenfalls weniger Aluminium (9-24 at.-\%). Außerdem weisen die Schichten eine sehr geringe Sauerstoffkonzentration von 0 bis 2 at.- $\%$ auf. Auch bei der Abscheidung auf $\mathrm{SiO}_{2}$ nimmt, wie bei der Deposition auf $\mathrm{MgO}$, die Kohlenstoffkonzentration durch zunehmende Carbidbildung mit steigender Substrattemperatur leicht zu (s. Abschnitt 6.2.1.1). Unabhängig davon ob der Ionenstrahl während der Deposition 
zugeschaltet wurde oder nicht, zeigen die Schichten bei gleicher Substrattemperatur die gleiche Elementzusammensetzung.

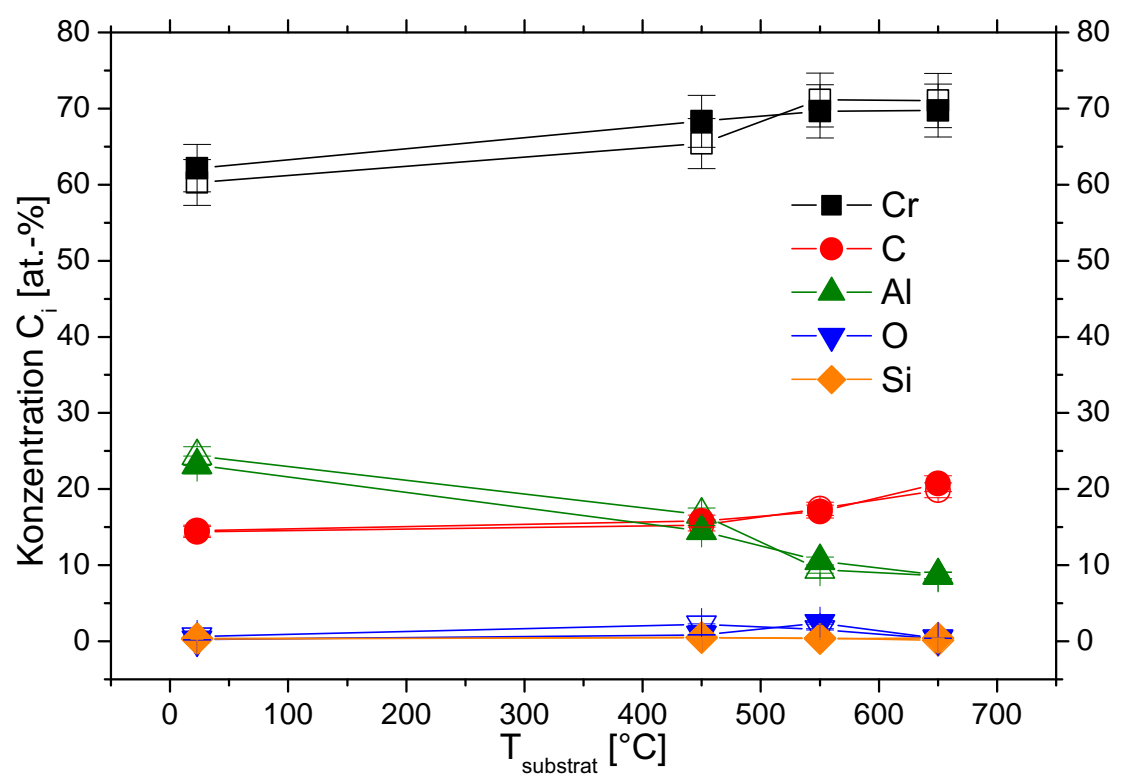

Abbildung 6.32: Mittlere Elementkonzentrationen $C_{i}$ der vom $\mathrm{Cr}_{2} \mathrm{AlC}$-Target auf $\mathrm{SiO}_{2}$ deponierten Schichten in Abhängigkeit der Substrattemperatur während der Ablation. Offene Symbole: ohne Ionenstrahl, geschlossene Symbole: mit zugeschaltetem $200 \mathrm{~V}$ Ar-Ionenstrahl. Die Linien dienen der besseren Orientierung.

Der Aluminiumgehalt der Schichten nimmt mit zunehmender Depositionstemperatur ab. Bei Raumtemperatur ist er am höchsten, und hier kommt es auch wieder zu der bei den MgO-Proben angesprochenen lokalen Erhöhung der Aluminiumkonzentration an der Grenzfläche zwischen Substrat und Film. Diese ist im Falle der Deposition mit Ionenstrahl erneut etwas stärker als bei der Ablation ohne Ionenstrahl. Wie in Abbildung $6.33 \mathrm{zu}$ sehen, ist die lokale Konzentrationserhöhung des Aluminium allerdings sehr viel weniger deutlich ausgeprägt als in den Schichten, die auf $\mathrm{MgO}$ deponiert wurden. Bei Temperaturen größer als Raumtemperatur tritt sie überhaupt nicht auf. Eine Diffusion von Elementen des $\mathrm{SiO}_{2}$-Substrats in die Schicht findet bei Raumtemperatur ebenfalls nicht statt.

Die bei den MgO-Proben beobachtete starke Inhomogenität des Aluminiumkonzentrationsprofils ist bei der Deposition auf $\mathrm{SiO}_{2}$ also nicht zu beobachten. Lediglich bei der Abscheidung bei Raumtemperatur kommt es zu einer leichten Erhöhung der Aluminiumkonzentration am Übergang von Substrat zur Schicht. In diesem Fall lässt sich wieder die in Abschnitt 6.2.1.2 postulierte Bildung der festen Lösung von Aluminium in Chrom 

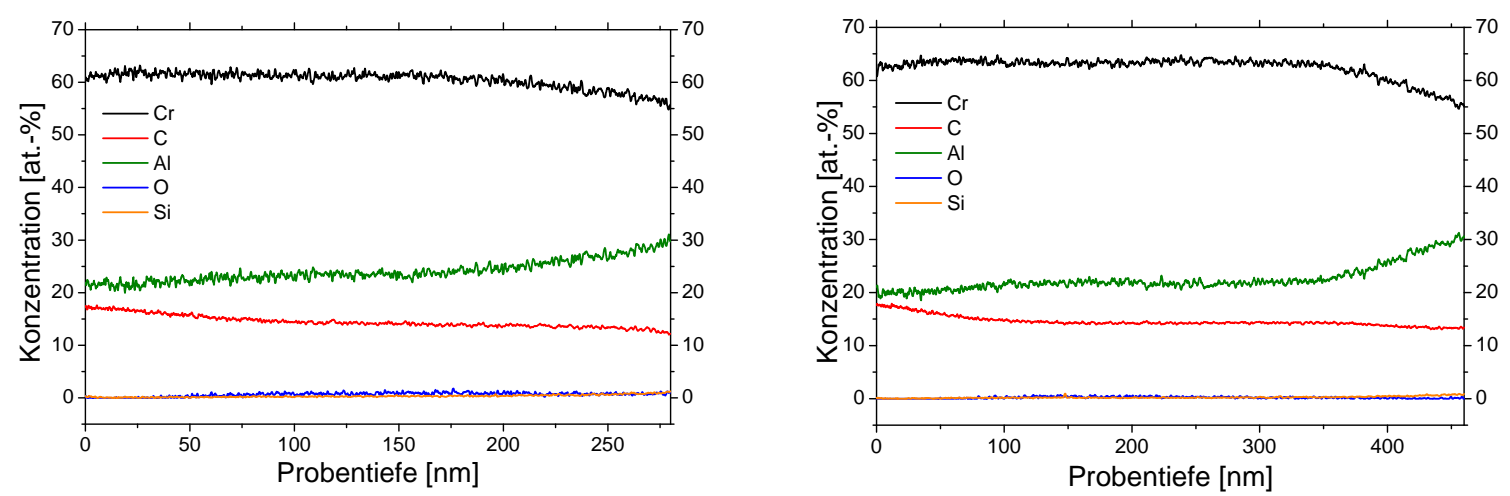

Abbildung 6.33: GDOES-Messungen zweier bei Raumtemperatur auf $\mathrm{SiO}_{2}$ deponierter $\mathrm{Cr} / \mathrm{Al} / \mathrm{C}-$ Schichten. Links: $\mathrm{T}=\mathrm{RT}$, ohne Ionenstrahl, rechts: $\mathrm{T}=\mathrm{RT}$, mit $200 \mathrm{~V}$ ArIonenstrahl. Das inhomogene Aluminium-Konzentrationsprofil mit der lokalen Anreicherung in Richtung der Substratoberfläche ist deutlich weniger ausgeprägt als bei den MgO-Proben.

vermuten, in der das Aluminium besser gebunden ist als in der Chromcarbid-Matrix. Allerdings fehlt im Fall der amorphen $\mathrm{SiO}_{2}$-Substratoberfläche die kristallographische Vorlage zur epitaktischen Bildung der tetragonalen intermetallischen Phase $\mathrm{Cr}_{2} \mathrm{Al}$. Eine durch Epitaxie bevorzugte Bildung von $\mathrm{Cr}_{2} \mathrm{Al}$ tritt folglich nicht auf. Statt dessen kommt es bei Temperaturen größer als RT von Anfang an zur Formation der Chromcarbid-Matrix, in der das Aluminium weniger stark gebunden ist, und die Aluminiumkonzentration ist durch die ganze Probe hindurch nahezu homogen auf einem Wert, der durch die Substrattemperatur gegebenen Gleichgewichtskonzentration aus Deposition und Absputtern entspricht (siehe Abb. 6.34). In der Abbildung ist außerdem zu erkennen, dass es bei hohen Temperaturen zu einer leichten Diffusion von Silizium aus dem Substrat in die Schicht kommt.

\subsubsection{Röntgendiffraktometrie}

Die Ergebnisse der Röntgendiffraktometrie für die $\mathrm{SiO}_{2}$-Schichten ähneln denen der auf $\mathrm{MgO}$ deponierten Proben. Die Beugungsbilder für die Deposition ohne Ionenstrahl sind in Abbildung 6.35 gezeigt. Auch hier sind für tiefere Temperaturen die Filme zunächst röntgenamorph. Bei Temperaturen ab $550{ }^{\circ} \mathrm{C}$ bildet sich dann das Chromcarbid $\mathrm{Cr}_{7} \mathrm{C}_{3}$. Die intermetallische Phase $\mathrm{Cr}_{2} \mathrm{Al}$ konnte im Fall der Deposition auf $\mathrm{SiO}_{2}$ nicht als kristalline Phase beobachtet werden. Wie bereits in Abschnitt 6.2.2.1 erwähnt, fehlt auf dem $\mathrm{SiO}_{2}$ auch die kubische Struktur, die als Vorlage für das epitaktische Wachstum von $\mathrm{Cr}_{2} \mathrm{Al}$ dient. Auffällig ist allerdings, dass die Beugungsbilder der $\mathrm{SiO}_{2}$-Proben bei Temperaturen $\geq 550{ }^{\circ} \mathrm{C}$ definierter sind als die der MgO-Proben. Die Zahl der Reflexe ist größer, 

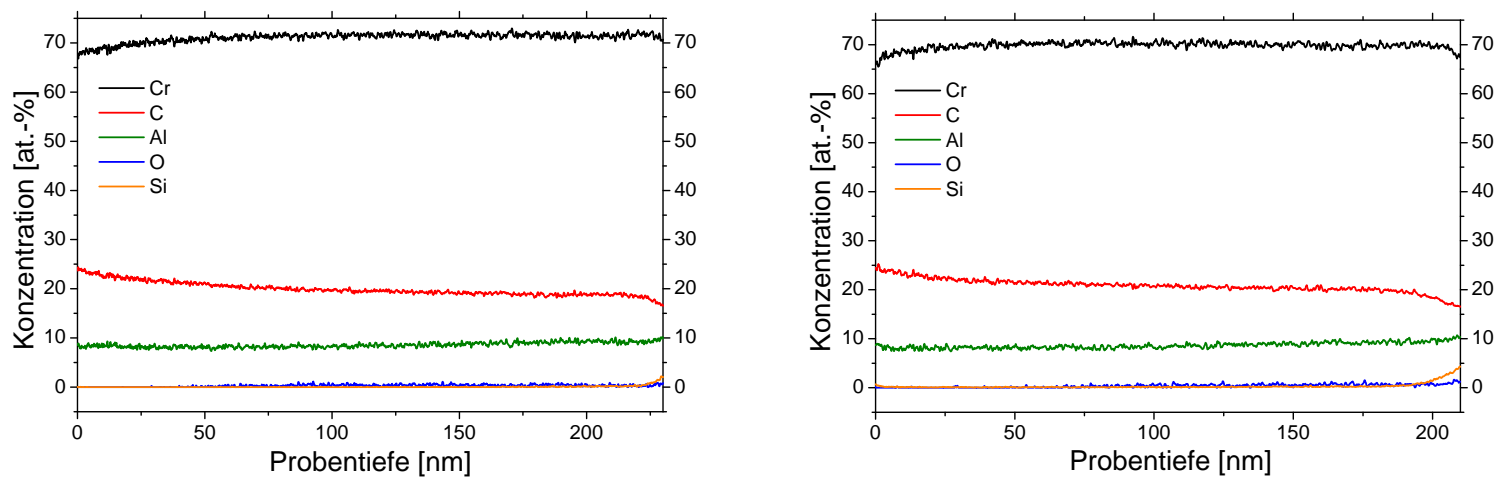

Abbildung 6.34: GDOES-Messungen zweier bei Raumtemperatur auf $\mathrm{MgO}(100)$ deponierter $\mathrm{Cr} / \mathrm{Al} / \mathrm{C}-S c h i c h t e n$. Links: $\mathrm{T}=650^{\circ} \mathrm{C}$, ohne Ionenstrahl, rechts: $\mathrm{T}=650^{\circ} \mathrm{C}$, mit $200 \mathrm{~V}$ Ar-Ionenstrahl. Das Aluminiumkonzentrationsprofil ist nahezu homogen. Es kommt zu keiner lokalen Erhöhung an der Grenzfläche zwischen Substrat und Schicht.

und gleichzeitig sind die Reflexe etwas schmaler als in Fall der Deposition auf MgO.

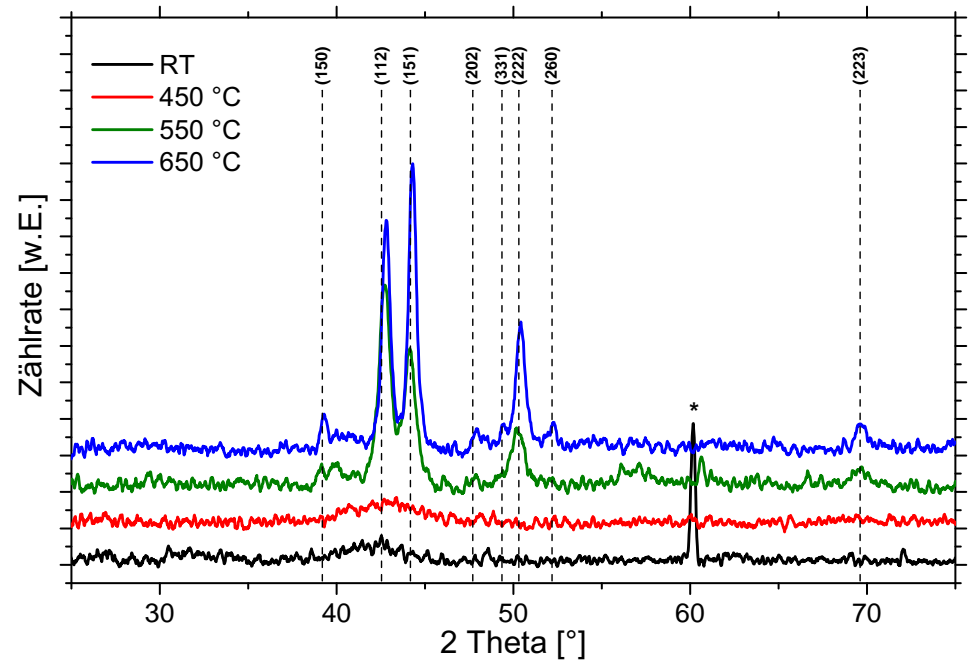

Abbildung 6.35: Röntgendiffraktogramme der $\mathrm{Cr}_{2} \mathrm{AlC}$-Proben auf $\mathrm{SiO}_{2}$ ohne Ionenstrahl. Abhängig von der Substrattemperatur kristallisiert die Phase $\mathrm{Cr}_{7} \mathrm{C}_{3}$, für die auch die (hkl)Indizes angegeben sind. Bei Raumtemperatur und $450{ }^{\circ} \mathrm{C}$ sind die Schichten röntgenamorph. Der scharfe mit einem Stern markierte Reflex ist ein Messartefakt, das vom Substrat stammt.

Bei der Deposition auf $\mathrm{SiO}_{2}$ mit Ionenstrahl (siehe Abb. 6.36) sind die sehr breiten Reflexe des amorphen - oder besser: nanokristallinen $-\mathrm{Cr}_{7} \mathrm{C}_{3}$ bei RT und $450{ }^{\circ} \mathrm{C}$ größer und deutlicher als bei der Abscheidung ohne Ionenstrahl. Bei beiden Depositionsmethoden (mit 
und ohne Ionenstrahl) sind bei Substrattemperaturen von $650{ }^{\circ} \mathrm{C}$ die gemessenen Beugungswinkel etwas größer als die in der Datenbank angegebenen (Strichlinien). Die Gitterebenenabstände $d$ in der Probe sind also kleiner als im $\mathrm{Cr}_{7} \mathrm{C}_{3}$-Vollmaterial im Gleichgewicht. Dies lässt entweder auf eine von $\mathrm{Cr}_{7} \mathrm{C}_{3}$ leint abweichende stöchiometrische Zusammensetzung oder auf intrinsische Spannungen in der Schicht schließen. Spannungen und Stress werden - aufgrund des Ungleichgewichtscharakters - sehr häufig in dünnen Schichten beobachtet. Kok et al. beschrieben bei der Deposition von dünnen Cr/C-Schichten intrinsischen Stress, der mit steigender Depositionstemperatur bis auf Werte von 2 GPa zunahm [153].

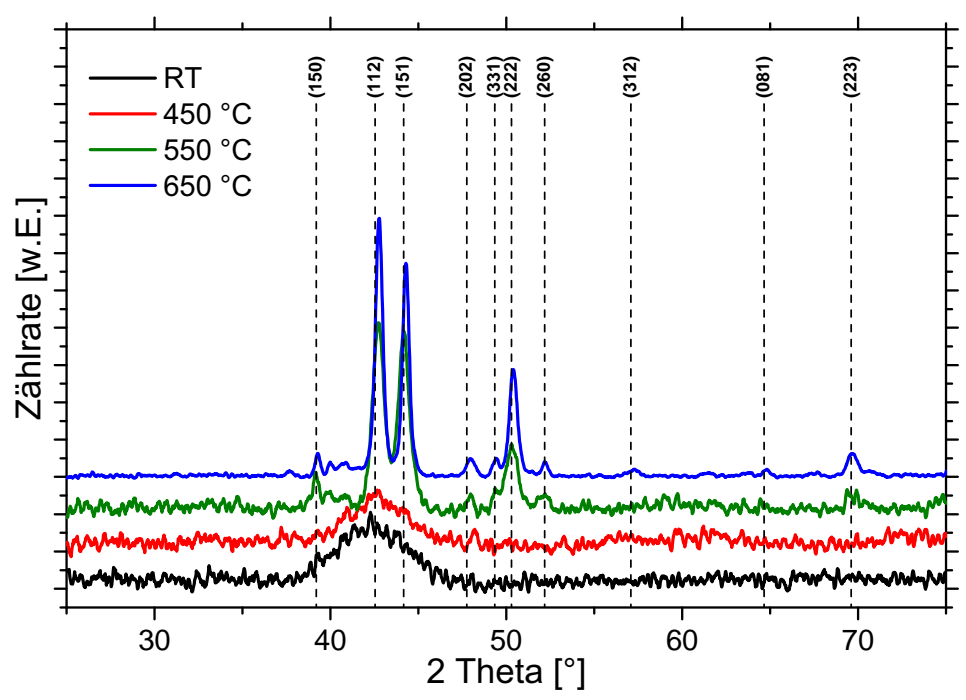

Abbildung 6.36: Röntgendiffraktogramme der $\mathrm{Cr}_{2} \mathrm{AlC}$-Proben auf $\mathrm{SiO}_{2}$ mit Ionenstrahl. In Abhängigkeit von der Substrattemperatur kristallisiert die Phase $\mathrm{Cr}_{7} \mathrm{C}_{3}$, deren (hkl)-Indizes ebenfalls angegeben sind. Bei Raumtemperatur und $450{ }^{\circ} \mathrm{C}$ sind die Schichten röntgenamorph.

\subsubsection{Härtemessungen}

Mit Härten von etwa 5 bis $6,5 \mathrm{GPa}$ sind die auf $\mathrm{SiO}_{2}$ deponierten Schichten weicher als die MgO-Proben. Wie Abbildung 6.37 zeigt, bleiben die Schichthärten als Funktion der Substrattemperatur in einem relativ engen Korridor. Nimmt man die Größe der Messfehler mit in Betracht, lässt sich kein signifikanter Einfluss der Depositionstemperatur auf die gemessenen Härten festellen. Auch die Auswirkung des Ionenstrahlbeschusses ist in diesem Fall nicht signifikant.

In den Ergebnissen der Röntgenbeugung war zu sehen, dass die $\mathrm{SiO}_{2}$-Proben überwiegend besser definierte Beugungsmuster als die MgO-Proben zeigten. Man kann also davon 


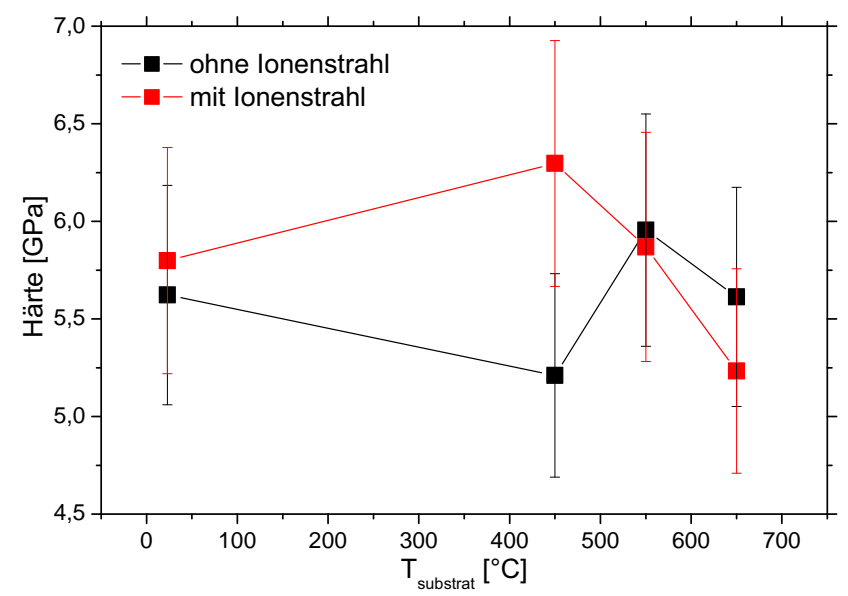

Abbildung 6.37: Schichthärten der auf $\mathrm{SiO}_{2}$ deponierten $\mathrm{Cr}_{2} \mathrm{AlC}-$ Proben (schwarz: ohne Ionenstrahl, rot: mit Ionenstrahl).

ausgehen, dass die Kristallite in den Proben größer sind, als in den auf $\mathrm{MgO}$ deponierten Schichten. Entsprechend der Hall-Petch-Beziehung führt dies zu einer geringeren Schichthärte, wenn die mittlere Kristallitgröße größer als die kritische Größe $d_{c}$ ist. Bei der Deposition auf $\mathrm{SiO}_{2}$ ist dies offensichtlich der Fall. Die deutliche Verringerung der Härte kann dadurch erklärt werden. Des Weiteren lässt sich auch ein - in Abschnitt 6.1.5 für die $\mathrm{Ti}_{3} \mathrm{SiC}_{2}$-Proben diskutierter - Einfluss der Substrathärte $\left(H_{\mathrm{SiO}_{2}} \approx 4 \mathrm{GPa}\right)$ nicht ausschließen. Allerdings sind die verwendeten Edelstahlsubstrate mit etwa $3 \mathrm{GPa}$ weicher als das $\mathrm{SiO}_{2}$-Substrat, während die auf Stahl deponierten Schichten - wie in späteren Abschnitten gezeigt - teilweise härter sind.

\subsubsection{Elektrische Eigenschaften}

Im Gegensatz zum System Ti-Si-C ist die MAX-Phase $\mathrm{Cr}_{2} \mathrm{AlC}$ nicht leitfähiger als die entsprechenden binären Chromcarbide. Die elektrische Leitfähigkeit von $\mathrm{Cr}_{3} \mathrm{C}_{2}$-Vollmaterial beträgt etwa $1,3 \cdot 10^{6}(\Omega \cdot \mathrm{m})^{-1}[152]$, die des $\mathrm{Cr}_{2}$ AlC-Vollmaterials $1,2 \cdot 10^{6}(\Omega \cdot \mathrm{m})^{-1}[155]$. Bei der Messung der elektrischen Leitfähigkeit von dünnen Cr/C-Schichten wurden von Maréchal et al. Werte zwischen $0,1 \cdot 10^{6}(\Omega \cdot \mathrm{m})^{-1}$ und $0,8 \cdot 10^{6}(\Omega \cdot \mathrm{m})^{-1}$ bestimmt. Diese Werte sind abhängig von der Sauerstoffkonzentration in den Schichten. Für nahezu sauerstofffreie Filme wurden die höchsten Leitfähigkeiten von $0,8 \cdot 10^{6}(\Omega \cdot \mathrm{m})^{-1}$ gemessen [152]. In Abbildung 6.38 sind die elektrischen Leitfähigkeiten der auf $\mathrm{MgO}$ und $\mathrm{SiO}_{2}$ deponierten Schichten gezeigt. In Abhängigkeit der Depositionsparameter liegen sie in einem Bereich von 0,4 bis $1,0 \cdot 10^{6}(\Omega \cdot m)^{-1}$. Dabei wurden die jeweils höchsten Werte bei einer Substrattemperatur von $650{ }^{\circ} \mathrm{C}$ gemessen. Die Verbesserung der kristallinen Qualität der Filme 
wirkt sich also positiv auf die elektrische Leitfähigkeit aus. Alle Kurven zeigen außerdem ein mehr oder weniger stark ausgeprägtes lokales Minimum bei $550{ }^{\circ} \mathrm{C}$. Die Röntgenbeugungsmessungen zeigen, dass etwa in diesem Temperaturbereich die Umwandlung von einem amorphen bzw. nanokristallinen Gefüge zu einer Struktur mit kristallinen Bereichen stattfindet. Es liegen folglich viele kleinere Kristallite mit einer erheblichen Anzahl von Korngrenzen vor, die die Leitfähigkeit absenken.

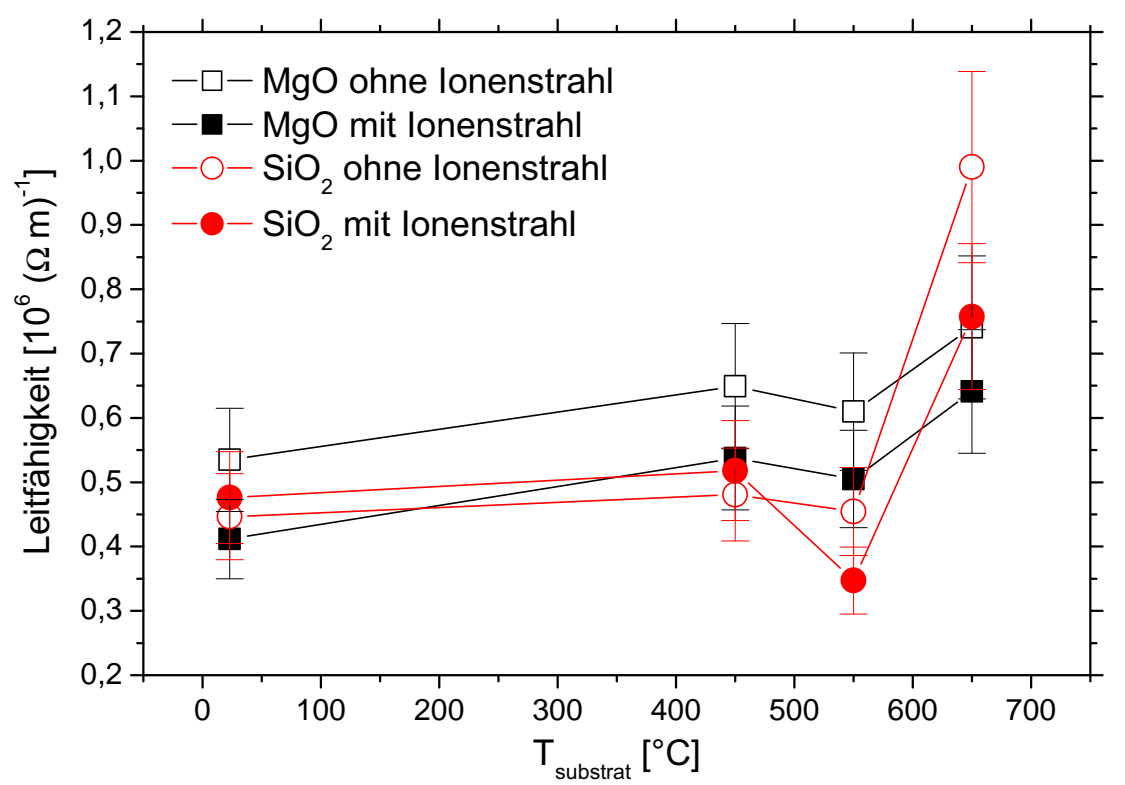

Abbildung 6.38: Leitfähigkeiten der auf $\mathrm{MgO}$ (schwarze Quadrate) und $\mathrm{SiO}_{2}$ (rote Kreise) deponierten $\mathrm{Cr} / \mathrm{Al} / \mathrm{C}-\mathrm{Schichten}$. Offene Symbole: ohne Ionenstrahl, geschlossene Symbole: mit Ionenstrahl.

\subsubsection{Edelstahlsubstrat}

\subsubsection{GDOES-Messungen}

Die Zusammensetzung der Cr/Al/C-Schichten auf den Edelstahlsubstraten ist vergleichbar mit denen auf $\mathrm{MgO}$ beziehungsweise $\mathrm{SiO}_{2}$. Das bedeutet, dass die mittleren Elementkonzentrationen aus den bereits diskutierten Gründen ebenfalls stark von der stöchiometrischen Komposition des $\mathrm{Cr}_{2} \mathrm{AlC}$-Targets abweichen. Der Chromgehalt ist erhöht und liegt abhängig von den Depositionsparametern zwischen 56 und 71 at.- \%. Bei Depositionstemperaturen oberhalb RT liegen die Aluminiumkonzentrationen deutlich unter 25 at.-\% in einem Bereich von etwa 8 bis 12 at.-\%. In den Raumtemperaturproben ist deutlich mehr 
Aluminium mit Anteilen von 18 at.-\% (ohne Ionenstrahl) und 27 at.-\% (mit Ionenstrahl) enthalten. Hier kommt es wiederum zu einer starken lokalen Anreicherung von Aluminium an der Grenzfläche zwischen Substrat und Film. Die Kohlenstoffkonzentration in den Schichten beträgt 13-19 at.-\%. Wie schon bei den $\mathrm{MgO}$ - und $\mathrm{SiO}_{2}$-Proben ist der Sauerstoffgehalt sehr niedrig und schwankt zwischen 0,3 und 1,1 at.-\% (ohne Ionenstrahl) bzw. 2,0 und 3,5 at.-\% (mit Ionenstrahl). Der leicht erhöhte Sauerstoffanteil bei der Deposition mit Ionenstrahl lässt sich durch Verunreinigungen im verwendeten Argongas und einer verstärkten Sauerstoffdiffusion in die Schichten durch die kinetische Energie der Ionen erklären.

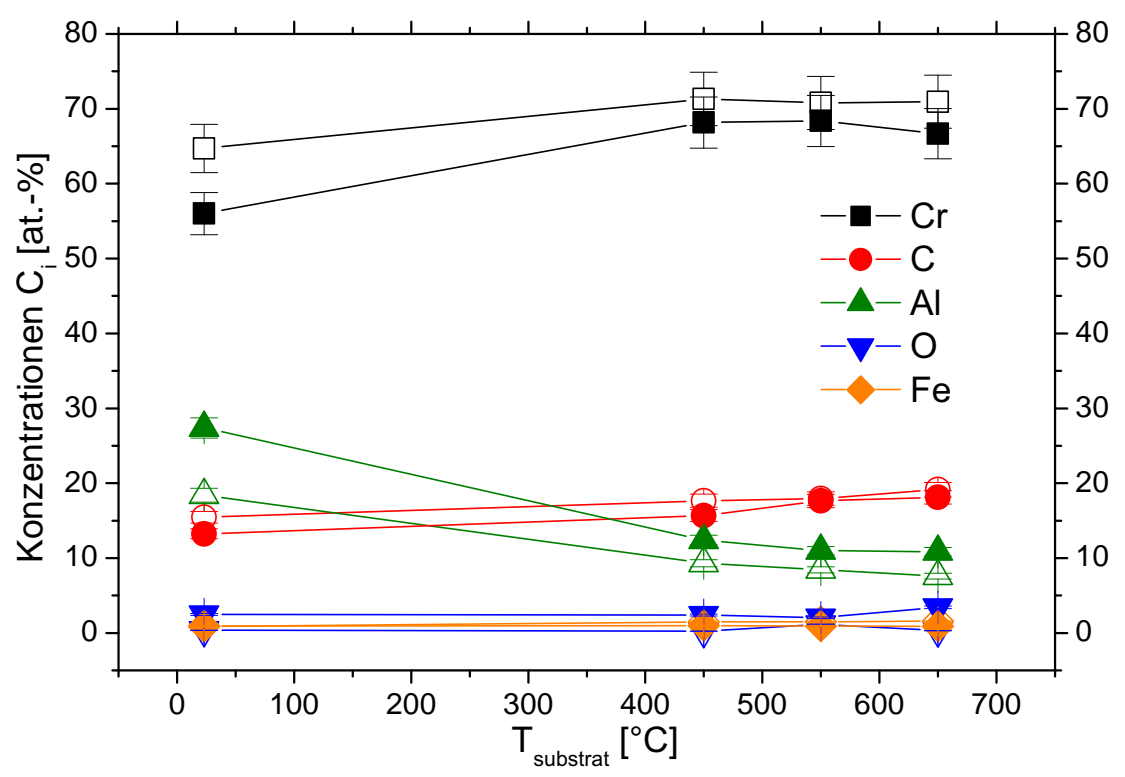

Abbildung 6.39: Mittlere Elementkonzentrationen $C_{i}$ der vom $\mathrm{Cr}_{2} \mathrm{AlC}$-Target auf Edelstahl deponierten Schichten in Abhängigkeit der Substrattemperatur während der Ablation. Offene Symbole: ohne Ionenstrahl, geschlossene Symbole: mit zugeschaltetem $200 \mathrm{~V}$ ArIonenstrahl. Die Linien dienen der besseren Orientierung.

Im Gegensatz zu den auf Edelstahl deponierten Ti/Si/C-Schichten kommt es im Fall der $\mathrm{Cr} / \mathrm{Al} / \mathrm{C}-\mathrm{Schichten}$ auch bei hohen Temperaturen und Ionenbeschuss nicht zu einer starken Diffusion von Eisen aus dem Substrat in den Film. Insgesamt liegen die Eisenkonzentrationen zwischen 0,9-1,5 at.-\% (ohne Ionenstrahl) und 0,9-1,0 at.-\% (mit Ionenstrahl). In Abbildung 6.40 sind die GDOES-Profile der bei $550{ }^{\circ} \mathrm{C}$ mit Ionenstrahl hergestellten Proben aus den Systemen $\mathrm{Cr} / \mathrm{Al} / \mathrm{C}$ und $\mathrm{Ti} / \mathrm{Si} / \mathrm{C}$ gegenüber gestellt. Deutlich zu erkennen ist, dass der Anstieg der Eisenkurve von der Schicht ins Substrat bei der $\mathrm{Cr} / \mathrm{Al} / \mathrm{C}$ Probe steiler ist als bei der Ti/Si/C-Probe. Die Diffusion von Eisen in den Film wird also stärker behindert. Da sowohl Substrate als auch Abscheidungsbedingungen in beiden 
Fällen gleich waren, muss der Grund in der chemischen Natur der Schichten selbst gesucht werden. Tatsächlich lässt sich die Unterdrückung der Eisendiffusion durch die Entstehung des Chromcarbids $\mathrm{Cr}_{7} \mathrm{C}_{3}$ (siehe XRD-Ergebnisse in Abschnitt 6.2.4.2) erklären. Chromcarbide reduzieren die Diffusion von Eisen aus Stahlsubstraten in dünne Schichten sehr effektiv. Dies wurde bereits von Schwarz et al. bei der Beschichtung von Edelstahl mit diamantartigen Schichten beschrieben [156].
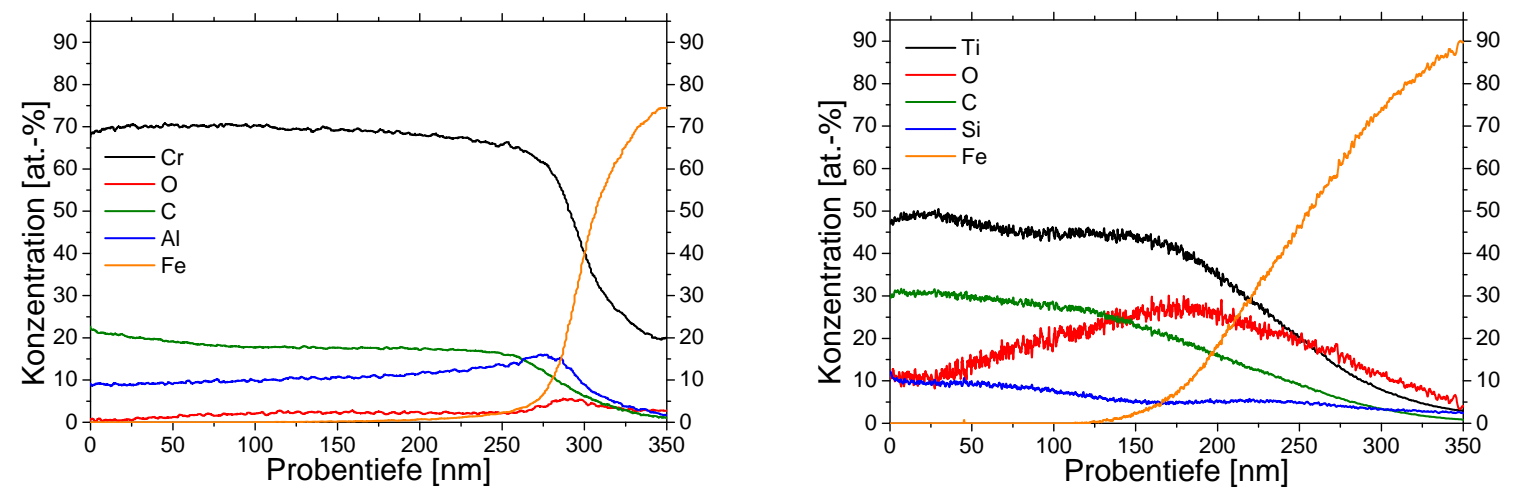

Abbildung 6.40: Vergleich der Eisendiffusion in einer $\mathrm{Cr} / \mathrm{Al} / \mathrm{C}$-Schicht (links) und einer $\mathrm{Ti} / \mathrm{Si} / \mathrm{C}-\mathrm{Schicht}$ (rechts). Beide Schichten wurden bei einer Substrattemperatur von $550{ }^{\circ} \mathrm{C}$ mit Ionenstrahlbeschuss hergestellt. Das Konzentrationsprofil des Eisens zeigt in der $\mathrm{Cr} / \mathrm{Al} / \mathrm{C}-$ Schicht einen deutlich steileren Anstieg. Die Diffusion von Eisen aus dem Substrat in die Schicht wird durch die Bildung des Chromcarbids $\mathrm{Cr}_{7} \mathrm{C}_{3}$ effektiv unterdrückt.

\subsubsection{Röntgendiffraktometrie}

Im Wesentlichen bestätigen die XRD-Ergebnisse der Edelstahlproben (s. Abbildungen 6.41 und 6.42) die Messungen auf den anderen beiden Substraten. Die Schichten sind - bei beiden Depositionsarten (ohne und mit Ionenstrahl) - bis $450{ }^{\circ} \mathrm{C}$ nur schwach kristallin. Bei höheren Temperaturen bildet sich das Chromcarbid $\mathrm{Cr}_{7} \mathrm{C}_{3}$. In den mit Ionenstrahl hergestellten Proben sind die Reflexe weniger deutlich ausgeprägt als in den ohne Ionenstrahl hergestellten. Der Ionenstrahlbeschuss verhindert also die Bildung größerer kristalliner Bereiche. Die intermetallische Phase $\mathrm{Cr}_{2} \mathrm{Al}$ bildet sich wiederum nicht. Grund dafür ist - wie im Fall der Abscheidung auf $\mathrm{SiO}_{2}$ - die fehlende kristallographische Vorlage des Substrates.

Ein wichtiger Unterschied zeigt sich jedoch in der bei $650{ }^{\circ} \mathrm{C}$ ohne Ionenstrahl hergestellten Probe. Hier sind zwar schwach aber dennoch eindeutig Beugungsreflexe eines weiteren Chromcarbids, nämlich $\mathrm{Cr}_{23} \mathrm{C}_{6}$ (ICSD 35-0783) zu sehen. Dieses Carbid stellt im binären 


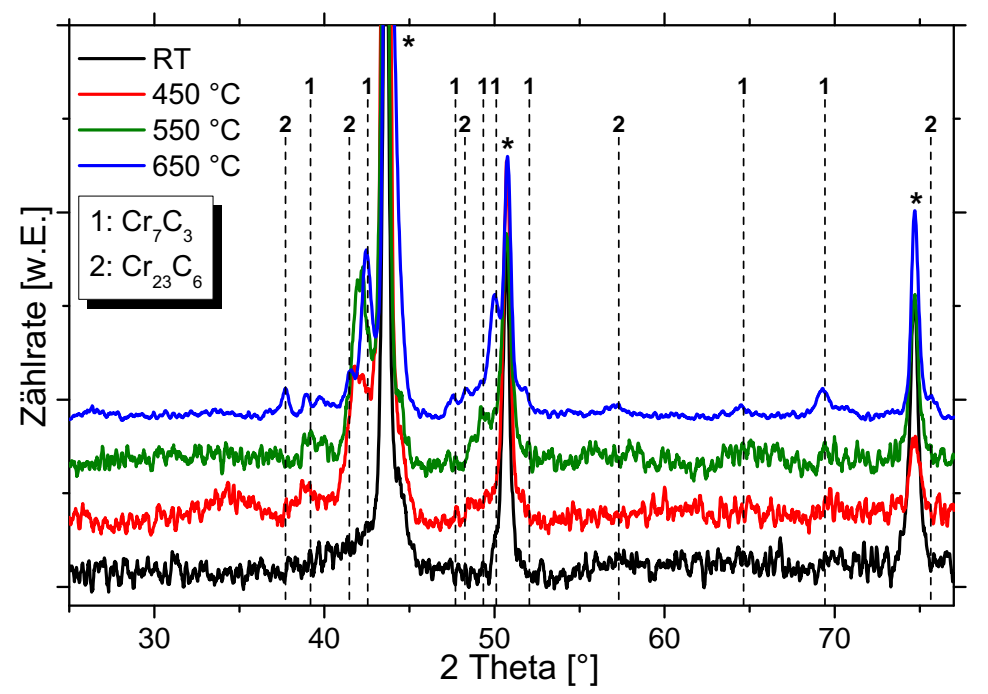

Abbildung 6.41: Röntgendiffraktogramme der $\mathrm{Cr}_{2} \mathrm{AlC}$-Proben auf Edelstahl ohne Ionenstrahl. Abhängig von der Substrattemperatur kristallisieren die Phasen $\mathrm{Cr}_{7} \mathrm{C}_{3}$ (1) und $\mathrm{Cr}_{23} \mathrm{C}_{6}$ (2). Bei Raumtemperatur ist die Schicht röntgenamorph. Die drei starken mit einem Stern markierten Reflexe stammen vom Edelstahlsubstrat.

Cr-C-Phasendiagramm die Nachbarphase $\mathrm{zu} \mathrm{Cr}_{7} \mathrm{C}_{3}$ in Richtung geringerer Kohlenstoffgehalt dar. Da die Chromcarbide einen sehr engen Homogenitätsbereich aufweisen, könnte die Bildung von $\mathrm{Cr}_{23} \mathrm{C}_{6}$ als Ausgleichsphase $\mathrm{zu} \mathrm{Cr}_{7} \mathrm{C}_{3}$ in allen Proben erwartet werden. Allerdings besitzt $\mathrm{Cr}_{23} \mathrm{C}_{6}$ eine sehr viel komplexere Kristallstruktur als $\mathrm{Cr}_{7} \mathrm{C}_{3}$ (s. Abb. 6.43). $\mathrm{Cr}_{7} \mathrm{C}_{3}$ kristallisiert in der orthorhombischen Raumgruppe Pnma mit 40 Atomen in der Elementarzelle. In ihr sind die Kohlenstoffatome von trigonalen Prismen aus sechs Chromatomen koordiniert. $\mathrm{Cr}_{23} \mathrm{C}_{6}$ hingegen bildet die kubische Raumgruppe $F m \overline{3} m$ mit 116 Atomen in der Elementarzelle, wobei sich eine Vielzahl von verschiedenen, miteinander verknüpften Koordinationspolyedern des Chroms um die Kohlenstoffatome bildet.

Zur Bildung dieser Struktur müssen die Atome sehr mobil sein, um die entsprechende Anordnung zu erreichen. So wurde auch in grundlegenden Arbeiten zum binären System Chrom-Kohlenstoff festgestellt, dass sich $\mathrm{Cr}_{23} \mathrm{C}_{6}$ nur bei sehr langsamer Abkühlung aus der Schmelze bildet $[157,158]$. Wird diese Bildung durch schnelles Abkühlen unterdrückt, verläuft die Phasenbildung nach:

$$
\mathrm{L} \leftrightarrow(\mathrm{Cr})+\mathrm{Cr}_{7} \mathrm{C}_{3}
$$

Aus der Schmelze bilden sich also nur das Carbid $\mathrm{Cr}_{7} \mathrm{C}_{3}$ und eine feste Lösung von Kohlenstoff in Chrom [59]. In dieser Hinsicht lassen sich mit PLD hergestellte dünne Schichten 


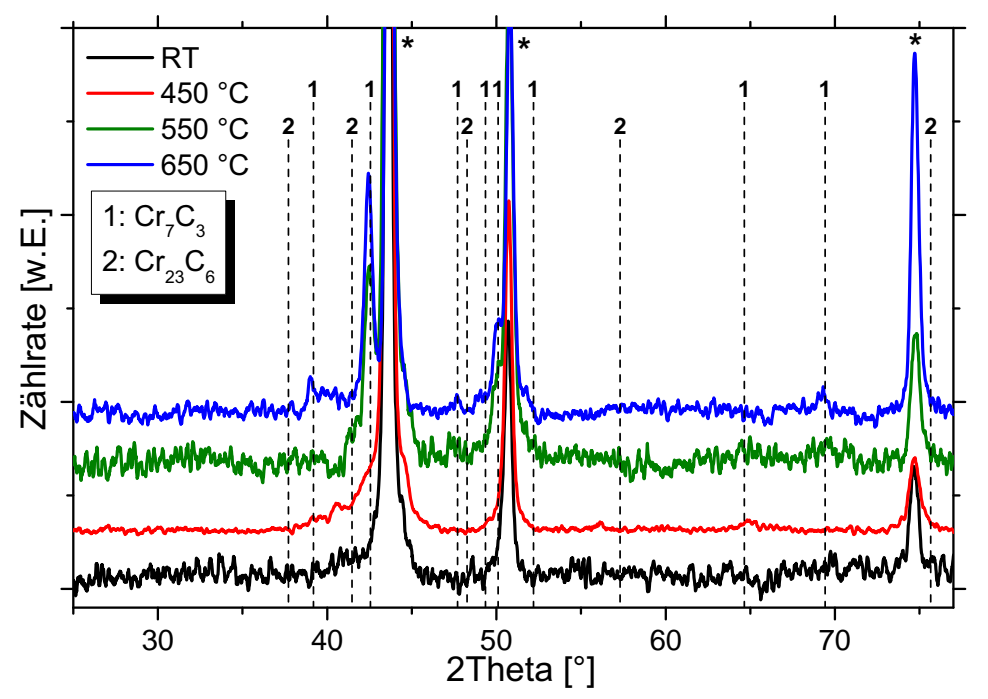

Abbildung 6.42: Röntgendiffraktogramme der $\mathrm{Cr}_{2} \mathrm{AlC}$-Proben auf Edelstahl mit Ionenstrahl. Abhängig von der Substrattemperatur kristallisiert die Phase $\mathrm{Cr}_{7} \mathrm{C}_{3}$ (1). Im Gegensatz zur Deposition ohne Ionenstrahl tritt die Bildung von $\mathrm{Cr}_{23} \mathrm{C}_{6}$ (2) nicht auf. Bei Raumtemperatur und $450{ }^{\circ} \mathrm{C}$ sind die Schichten röntgenamorph. Die drei starken mit einem Stern markierten Reflexe stammen vom Edelstahlsubstrat.

aufgrund ihrer Ungleichgewichtsnatur eher mit schnell abgekühlten Schmelzen vergleichen. Dass das Chromcarbid $\mathrm{Cr}_{23} \mathrm{C}_{6}$ in nahezu allen Proben nicht auftritt, lässt sich somit sehr einfach erklären. Lediglich bei der $650^{\circ} \mathrm{C}$-Deposition ohne Ionenstrahl lagen offensichtlich Bedingungen vor, die die Bildung von wenigstens geringen Mengen kristallinen $\mathrm{Cr}_{23} \mathrm{C}_{6}$ ermöglichten.

(a)

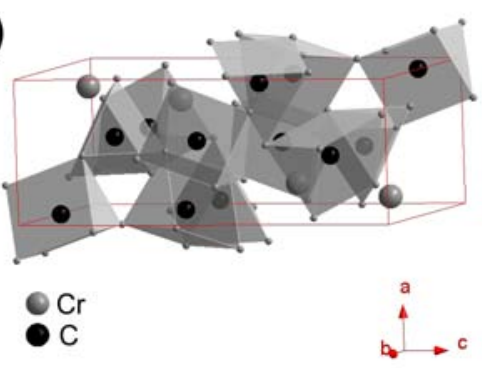

(b)

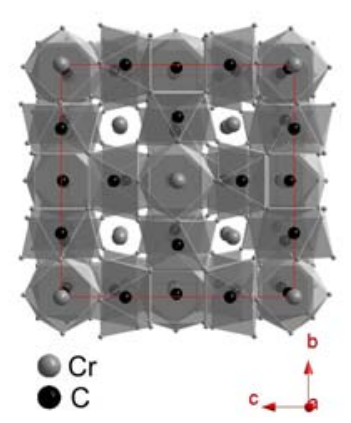

Abbildung 6.43: Vergleich der Elementarzellen von (a) $\mathrm{Cr}_{7} \mathrm{C}_{3}$ und (b) $\mathrm{Cr}_{23} \mathrm{C}_{6}$. In $\mathrm{Cr}_{7} \mathrm{C}_{3}$ sind die Kohlenstoffatome von trigonalen Prismen aus Chromatomen umgeben. $\mathrm{Cr}_{23} \mathrm{C}_{6}$ besitzt eine wesentlich komplexere Elementarzelle mit 116 Atomen und einer Vielzahl verschiedener, miteinander verknüpfter Koordinationspolyeder. 


\subsubsection{Härtemessungen}

Verglichen mit den beiden anderen Substraten sind die gemessenen Schichthärten der $\mathrm{Cr}_{2} \mathrm{AlC}$-Proben geringer als bei der Deposition auf $\mathrm{MgO}$, aber höher als bei der Deposition auf $\mathrm{SiO}_{2}$. Die entsprechenden Messwerte sind in Abbildung 6.44 gezeigt. Im Gegensatz zu den anderen $\mathrm{Cr}_{2} \mathrm{AlC}$-Depositionen treten bei den Edelstahlproben erhebliche Unterschiede in der Härte bei der Abscheidung mit und ohne Ionenstrahl auf. Bei Raumtemperatur beträgt die Härte in beiden Fällen etwa 7 GPa. Wird kein Ionenstrahl zugeschaltet, steigen die Schichthärten mit der Substrattemperatur auf Werte von 8,5 bis 9,5 GPa an. Bei der Verwendung des Ionenstrahls nehmen die gemessenen Härten dagegen kontinuierlich bis auf einen Wert von $4,5 \mathrm{GPa}$ bei $650{ }^{\circ} \mathrm{C}$ ab.

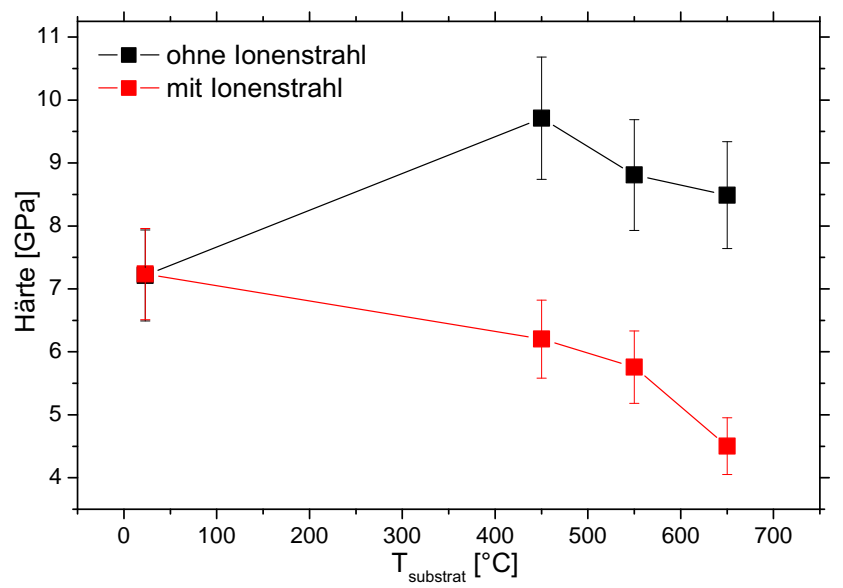

Abbildung 6.44: Schichthärten der auf Edelstahl deponierten $\mathrm{Cr}_{2} \mathrm{AlC}-P r o b e n$ (schwarz: ohne Ionenstrahl, rot: mit Ionenstrahl).

Eine Diskussion entsprechend der Hall-Petch-Beziehung lässt sich in diesem Fall also nicht unbedingt führen. Betrachtet man die Proben, die ohne Ionenstrahl deponiert wurden, steigt die Schichthärte zunächst an, fällt bei Temperaturen $\mathrm{T} \geq 550{ }^{\circ} \mathrm{C}$ aber wieder ab. Wie die XRD-Messungen gezeigt haben, entsteht bei hohen Temperaturen eine zweite kristalline Carbidphase $\mathrm{Cr}_{23} \mathrm{C}_{6}$. Die Entstehung weiterer Phasen in einer dünnen Schicht führt aber zu zusätzlichen Inhomogenitäten, die die Härte absenken [143, 159]. Bei der Deposition mit Ionenstrahl kommt es dagegen zu einer kontinuierlichen Abnahme der Schichthärte, obwohl auch hier die Beugungsreflexe mit zunehmender Substrattemperatur deutlicher werden. Die GDOES-Messungen zeigen bei $650{ }^{\circ} \mathrm{C}$ den höchsten Sauerstoffgehalt von 3,5 at.-\%, was zum Teil zur Verringerung der Schichthärte beiträgt. Aus den XRD-Messungen lässt sich erkennen, dass der Anteil von kristallinen Bereichen geringer ist, als in den ohne Ionenstrahl abgeschiedenen Schichten. Daraus folgt ein relativ 
großer Anteil an amorphen Bereichen in der Schicht. Dies kann aber nur die insgesamt geringere Schichthärte im Vergleich zu den ohne Ionenstrahl deponierten Filmen erklären. Warum es aber zu der kontinuierlichen Abnahme der Härte kommt, ist nicht klar. Weitere Untersuchungen zur Klärung dieses Phänomens sind deswegen notwendig.

\subsubsection{Zusammenfassung - $\mathrm{Cr}_{2}$ AIC-System}

Auch bei der ionenstrahlunterstützten Pulslaserdeposition von einem $\mathrm{Cr}_{2} \mathrm{AlC}$-Target gelang die Herstellung homogener und rissfreier Schichten. Im Gegensatz zu den Ergebnissen im System Ti-Si-C kam es hierbei - trotz gleicher Vakuumbedingungen - nicht zu einer nennenswerten Sauerstoffaufnahme in die Schichten. Die Elemente Chrom und Aluminium zeigten sich unter den Depositionsbedingungen weniger sauerstoffaffin als die Elemente Titan und Silizium. In Abhängigkeit von der Substrattemperatur während der Deposition wiesen alle Proben leicht veränderte Elementzusammensetzungen auf, die allerdings in keinem Fall der stöchiometrischen Zusammensetzung des $\mathrm{Cr}_{2} \mathrm{AlC}$-Targets entsprachen. Die in den Schichten erhaltenen Zusammensetzungen repräsentieren den stationären QuasiGleichgewichtszustand aus Deposition und Absputtern bzw. Abdampfen während des Abscheidungsprozesses. Es kam in aller Regel zu einem hohen Verlust an Aluminium. Die Kohlenstoffkonzentration nahm bei steigender Substrattemperatur leicht zu, da es zu einer vermehrten Carbidbildung in den Schichten kam. Zusätzlich kommt es bei der PLD aus dem $\mathrm{Cr}_{2} \mathrm{AlC}$-Target in vielen Fällen zu einer inhomogenen Verteilung des A-Elementes Aluminium in der Schicht.

Grund dafür ist die Bildung einer festen Lösung von Aluminium in Chrom in der Nähe der Substratoberfläche. In dieser festen Lösung ist das Aluminium besser gebunden als in der sich später bildenden Chromcarbid-Matrix. In einigen Fällen kam es sogar zu einer lokalen Anreicherung des Aluminiums zu Konzentrationen größer als die 25 at.-\% Al im Target. Hier wurde durch Diffusionsprozesse also noch mehr Aluminium im Chrom gelöst. Die Zuschaltung des Argon-Ionenstrahls während der Abscheidung verstärkt diesen lokalen Anreicherungseffekt. Gibt man durch das Substrat eine entsprechenden kristallographische Vorlage vor - in dieser Arbeit ist es das MgO-Substrat mit seiner kubischen

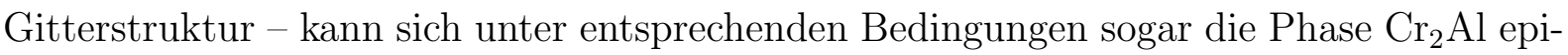
taktisch bilden, was durch Röntgenbeugungs- und TEM-Messungen bestätigt wurde.

Die Bildung der $\mathrm{Cr}_{2}$ AlC-MAX-Phase konnte in keinem Fall nachgewiesen werden. Entsprechend der engen strukturellen Verwandschaft der MAX-Phasen mit den binären MXVerbindungen kam es statt dessen zur Bildung von Chromcarbiden. Da das binäre 1:1Carbid CrC nur metastabil ist, trat in diesem Fall das Chromcarbid $\mathrm{Cr}_{7} \mathrm{C}_{3}$ in Form von kristallinen Bereichen in einer amorphen Umgebung auf. Im thermischen Gleichgewicht sollte als Ausgleichsphase für geringere Kohlenstoffkonzentrationen ebenfalls das Carbid 
$\mathrm{Cr}_{23} \mathrm{C}_{6}$ entstehen. Aufgrund der sehr viel komplexeren Kristallstruktur ist dessen Bildung allerdings kinetisch gehemmt. Statt dessen kommt es - wie auch bei der schnellen Abkühlung aus der Schmelze - lediglich zur Bildung einer festen Lösung von Kohlenstoff in Chrom. Folglich wurden nur in einer Probe geringe Mengen von $\mathrm{Cr}_{23} \mathrm{C}_{6}$ nachgewiesen. Eine interessante Eigenschaft der Chromcarbide - ihre effektive Wirkung als Diffusionsbarriere - ließ sich bei der Schichtherstellung auf Edelstahl beobachten. Während die Edelstahlproben im $\mathrm{Ti}_{3} \mathrm{SiC}_{2}$-System insbesondere bei hohen Temperaturen und der Benutzung des Ionenstrahls sehr stark durch die Diffusion von Eisen aus dem Substrat in die Schicht geprägt waren, trat bei den Schichten im $\mathrm{Cr}_{2} \mathrm{AlC}$-System diese Diffusion kaum auf.

Bei der Betrachtung makroskopischer Eigenschaften wie elektrische Leitfähigkeit und Härte zeigen die Proben ein Verhalten, das dem von reinen Chrom/Kohlenstoff-Schichten entspricht. So liegen die elektrischen Leitfähigkeiten mit Werten von 0,5-1,0 $\cdot 10^{6}(\Omega \cdot \mathrm{m})^{-1}$ etwas unterhalb derer des Chromcarbid-Vollmaterials aber trotzdem in einem Bereich, der auch für andere dünne Schichten aus Chrom und Kohlenstoff gemessen wurde. Die Schichthärten schwanken je nach verwendetem Substrat sehr stark. Auf dem MgO-Substrat mit seiner gut definierten Oberfläche betragen sie bis zu 11,5 GPa. Diese Härten sind in Übereinstimmung mit anderen an dünnen Chromcarbid-Schichten gemessenen Schichthärten. Auf den relativ rauen, aber dennoch über eine kristalline Oberfläche verfügenden Edelstahlsubstraten sind die Schichten etwas weicher. Drastisch verringerte Schichthärten zeigen sich auf der amorphen Oberfläche der $\mathrm{SiO}_{2}$-Substrate. Die Beschaffenheit der Substratoberfläche wirkt sich also stark auf die atomare Struktur der Filme und damit auch auf ihre Härte aus. 


\section{Ergebnisse der reaktiven PLD}

Die in diesem Kapitel dargestellten Ergebnisse beschreiben die ersten Arbeiten zur reaktiven PLD im MAX-Phasen-System Ti-Al-N. Dazu wurde ein aus den reinen Metallen Titan und Aluminium im Verhältnis 2:1 zusammengesetztes Target ablatiert, während gleichzeitig mit der an der Kammer befindlichen Ionenquelle ein Stickstoff-Ionenstrahl zur chemischen Reaktion auf das Substrat gerichtet wurde. Dabei wurden Ionenbeschleunigungsspannungen von 0 bis $1000 \mathrm{~V}$ angelegt. Die Substrate waren in allen Fällen einkristalline $\mathrm{MgO}(100)-$ Scheiben. Die Substrattemperaturen während der Deposition lagen entweder bei $450{ }^{\circ} \mathrm{C}$ oder bei $650{ }^{\circ} \mathrm{C}$. Alle Proben zeigten äußerlich eine mehr oder weniger starke goldene Färbung, was auf die Bildung von Titannitrid TiN hindeutet.

Eine genaue Charakterisierung der Schichten gestaltet sich relativ schwierig. So sind alle Schichten röntgenamorph. Eine Identifikation kristalliner Phasen ist also nicht möglich. Dafür können einige Faktoren als Erklärung herangezogen werden. Wie im Folgenden beschrieben, sind die Schichten sehr inhomogen in ihrer chemischen Zusammensetzung. Durch die massive Inkorporation von Sauerstoff entstehen neben nitridischen auch oxidische Verbindungen. Dieses Gemisch aus verschiedenen chemischen Verbindungen verhindert eine Kristallisation der einzelnen Phasen. Die Hemmung der Kristallisation von Nitriden durch Oxide in der Schicht wurde bei der reaktiven PLD von Titannitrid von Mihailescu et al. bereits gezeigt [102]. Des Weiteren kann auch der Ionenstrahl selbst eine Kristallisierung verhindern. Durch die teilweise hohe kinetische Energie der Stickstoffionen und die mit etwa $15^{\circ}$ ziemlich große Divergenz des Strahls können eher Strukturschäden in der Schicht induziert werden, als dass eine - wie in Abschnitt 2.2.4 geschilderte gezielte Beeinflussung des kristallinen Wachstums auftritt.

Wie bereits erwähnt, zeigen die GDOES-Ergebnisse sehr ungleichmäßige Elementverteilungen in den Schichten. Dabei enthielten die Filme Titan, Aluminium, Stickstoff und relativ große Mengen Sauerstoff. Dazu kommen ein sehr geringer $(\leq 1,5$ at.- $\%)$ Anteil an Kohlenstoff, der als Verunreinigung durch den Ionenstrahl in die Proben gelangte, sowie geringe Mengen Magnesium durch Diffusion an der Grenzfläche zwischen Substrat und Film. Ein genauerer Blick auf die Ergebnisse der GDOES-Messungen soll im folgenden Abschnitt gegeben werden. 


\subsection{GDOES-Ergebnisse}

\subsubsection{Mittlere Elementkonzentrationen $C_{i}$}

In den Abbildungen 7.1 und 7.2 sind für die beiden Depositionstemperaturen $450{ }^{\circ} \mathrm{C}$ bzw. $650{ }^{\circ} \mathrm{C}$ die mittleren Elementkonzentrationen $C_{i}$ als Funktion der Beschleunigungsspannung des Stickstoffionenstrahls angegeben. Bei einer Substrattemperatur von $450{ }^{\circ} \mathrm{C}$ enthalten die Schichten - abhängig von der angelegten Spannung - 24-30 at.-\% Titan, einen zwischen 17 und 18 at.-\% relativ konstanten Anteil von Aluminium, sowie 1939 at.-\% Stickstoff. Der Sauerstoffanteil in den Schichten schwankt zwischen 16 und 29 at.-\%. Zu erklären ist die hohe Sauerstoffkonzentration durch mangelnde Reinheit des verwendeten Stickstoffgases, durch die gleichzeitig der hoch reaktive Sauerstoff in die Kammeratmosphäre eingebracht wird. Außerdem zeigt Titan eine sehr hohe Affinität zu Sauerstoff, wie schon bei den Versuchen zur PLD des $\mathrm{Ti}_{3} \mathrm{SiC}_{2}$-Targets (s. Abschnitt 6.1) gezeigt wurde. Die Kohlenstoffverunreinigungen und die Magnesiumdiffusion sind vernachlässigbar klein. Die größten Unterschiede in der elementaren Zusammensetzung der Schichten treten auf, wenn eine Beschleunigungsspannung größer $0 \mathrm{~V}$ angelegt wird. Das heißt, der Stickstoff strömt nicht nur als Hintergrundgas in die Kammer, sondern wird als Ionenstrahl auf das Substrat gerichtet. In diesem Fall nimmt der Stickstoffgehalt der Proben von 19 auf über 35 at.-\% sprunghaft zu. Gleichzeitig nimmt die Sauerstoffkonzentration in einem vergleichbaren Maß ab. Auch der Titangehalt sinkt von 30 auf etwa 25 at.-\%. Der Anteil an Aluminium hingegen bleibt - bei allen Beschleunigungsspannungen - in etwa gleich. Bereits bei der Deposition ohne Beschleunigung der Ionen wird das im Target vorliegende Mengenverhältnis von Ti:Al = 2:1 nicht mehr in der Schicht erhalten. Es liegt tatsächlich bei 1,8:1. Wird eine Beschleunigungsspannung angelegt, sinkt der Titangehalt in den Schichten, und das Ti:Al-Verhältnis nimmt weiter ab. Das Titan wird also im Vergleich zum Aluminium von den ankommenden Teilchen bevorzugt aus der Schicht herausgesputtert. Des Weiteren kann man feststellen, dass sich die Konzentrationen von Stickstoff und Sauerstoff gegenläufig verhalten. Nimmt an einer Stelle der Stickstoffgehalt zu, nimmt der Sauerstoffgehalt gleichzeitig ab. Offensichtlich kommt es in den Schichten also zu konkurrierenden Oxidations- und Nitridationsprozessen.

Ein ähnliches Verhalten zeigen die bei $650{ }^{\circ} \mathrm{C}$ deponierten Schichten. Auch hier hat die Titankonzentration ihr Maximum von 34 at.-\% bei der Deposition ohne Beschleunigungsspannung und nimmt danach auf Werte von 23-28 at.-\% ab. Der Aluminiumgehalt bleibt - unabhängig von der Beschleunigungsspannung - konstant bei etwa 16-18 at.-\%. Der Stickstoffanteil in den Proben ist mit 18-42 at.-\% ein wenig größer als in den Proben, die bei $450{ }^{\circ} \mathrm{C}$ deponiert wurden, während die $650{ }^{\circ} \mathrm{C}$-Proben mit 13-25 at.- $\%$ etwas weniger Sauerstoff enthalten. Sowohl das deutliche Ansteigen der Stickstoffkonzentration 


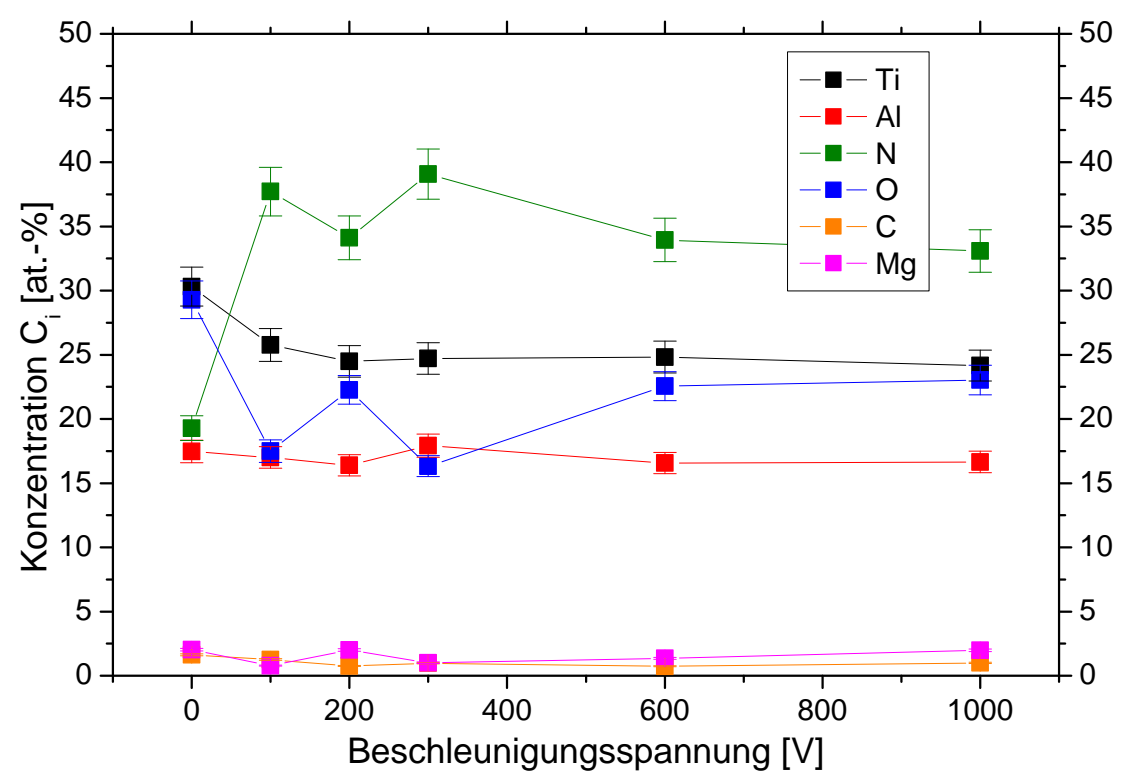

Abbildung 7.1: Mittlere Elementkonzentrationen $C_{i}$ der bei $450{ }^{\circ} \mathrm{C}$ auf $\mathrm{MgO}(100)$ deponierten Ti-Al-N-Schichten als Funktion der Beschleunigungsspannung des Stickstoffionenstrahls. Die Linien dienen der besseren Orientierung.

bei Anlegen der Beschleunigungsspannung als auch die Konkurrenz zwischen Oxidation und Nitridation sind auch bei der Substrattemperatur $\mathrm{T}=650{ }^{\circ} \mathrm{C}$ erkennbar.

\subsubsection{Elementprofile}

Wie bereits weiter oben angedeutet sind die einzelnen Elemente in den Schichten sehr inhomogen verteilt. Besonders deutlich zeigt sich dies in den Filmen, die ohne einen gerichteten Ionenstrahl hergestellt wurden. Die GDOES-Profile dieser beiden Proben $\left(450{ }^{\circ} \mathrm{C}\right.$ und $650{ }^{\circ} \mathrm{C}$ ) sind in Abbildung 7.3 dargestellt. Die Konzentrationskurven aller Elemente zeigen zum Teil starke Sprünge bei etwa zwei Drittel der Schichtdicke. Der Sauerstoffgehalt nimmt in Richtung der Schichtoberfläche drastisch ab, während die Anteile von Titan und Stickstoff zunehmen. Ebenso wie die Sauerstoffkonzentration, aber in geringerem Ausmaß, nimmt auch die Aluminiumkonzentration an dieser kritischen Stelle sprunghaft ab.

Vergleicht man die GDOES-Profile ohne Ionenstrahl mit denen bei angelegter Beschleunigungsspannung, lassen sich gewisse Ähnlichkeiten, aber auch Unterschiede feststellen. Sowohl die Substrattemperatur als auch die Energie der eingestrahlten Ionen haben einen Einfluss auf die Form der Elementverteilungsprofile. In Abbildung 7.4 sind die GDOESProfile für jeweils $450{ }^{\circ} \mathrm{C}$ und $650{ }^{\circ} \mathrm{C}$ bei $200 \mathrm{~V}$ und $1000 \mathrm{~V}$ Beschleunigungsspannung 


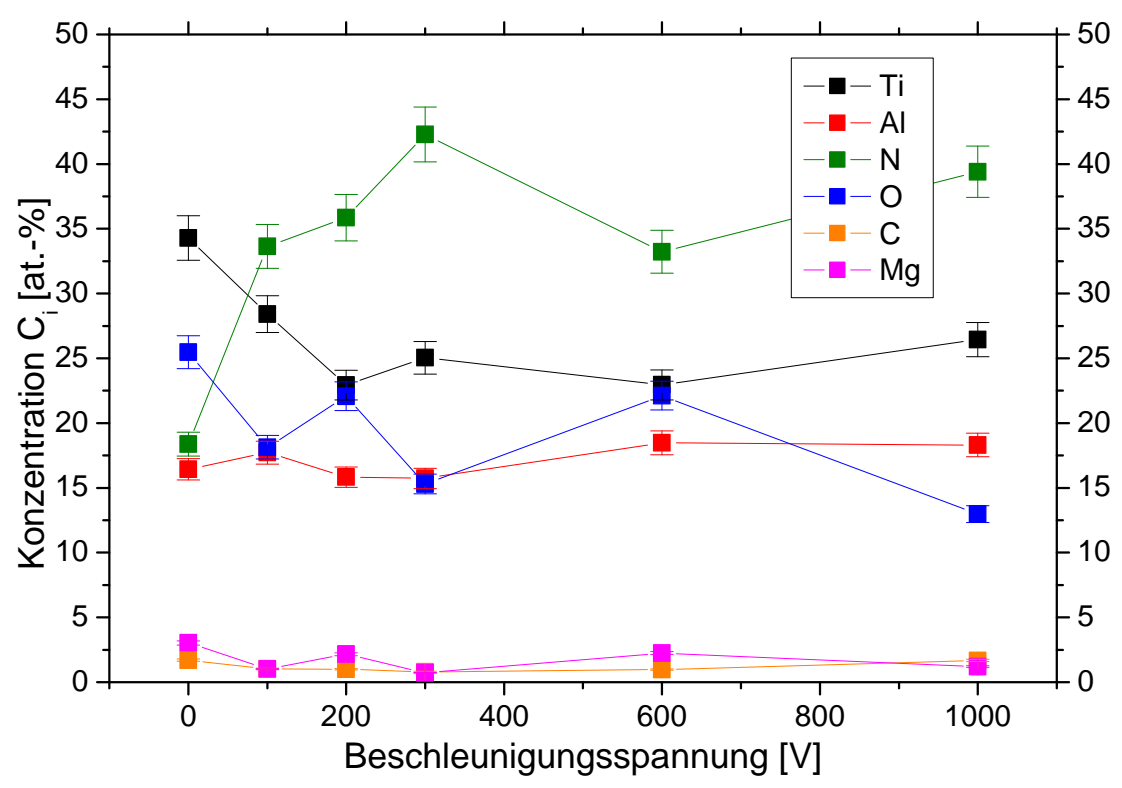

Abbildung 7.2: Mittlere Elementkonzentrationen $C_{i}$ der bei $650{ }^{\circ} \mathrm{C}$ auf $\mathrm{MgO}(100)$ deponierten Ti-Al-N-Schichten als Funktion der Beschleunigungsspannung des Stickstoffionenstrahls. Die Linien dienen der besseren Orientierung.
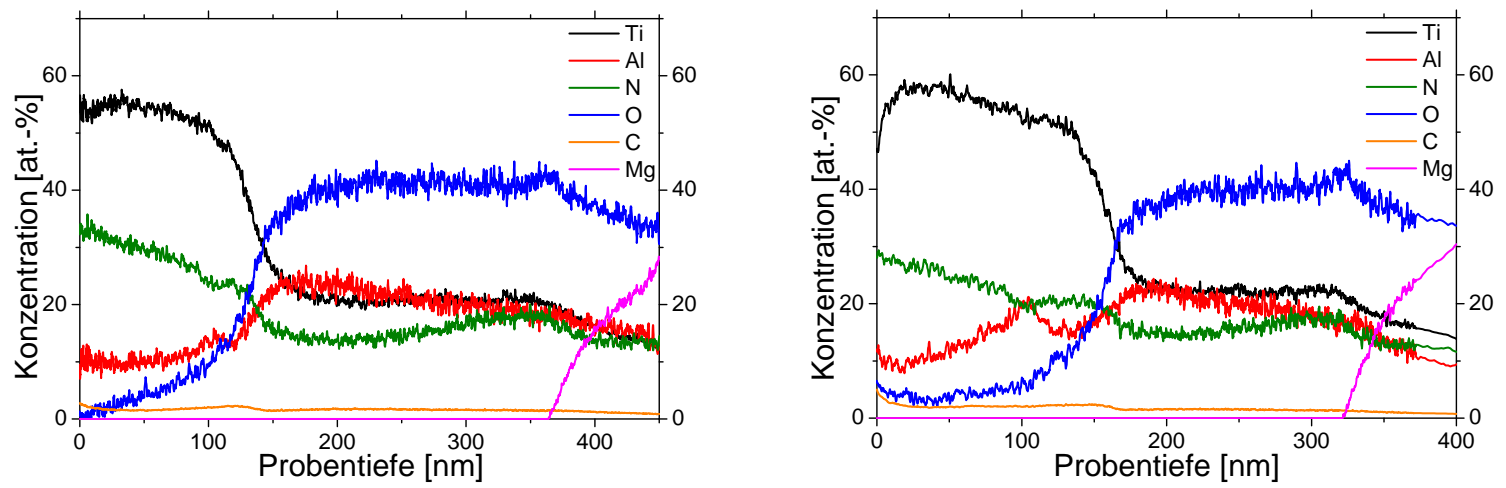

Abbildung 7.3: GDOES-Messungen zweier ohne Beschleunigungsspannung auf $\mathrm{MgO}(100)$ deponierter $\mathrm{Ti} / \mathrm{Al} / \mathrm{N}-\mathrm{Schichten}$. Links: $\mathrm{T}=450{ }^{\circ} \mathrm{C}$, rechts: $\mathrm{T}=650{ }^{\circ} \mathrm{C}$. Durch eine Umschaltung in der Messwertaufnahme des Gerätes werden die Kurven im rechten Teilbild zum Ende hin geglättet.

gegenüber gestellt. Bei $200 \mathrm{~V}$ und $450{ }^{\circ} \mathrm{C}$ (Abb. 7.4 (a)) ist die deutliche Konzentrationsänderung der Elemente noch sehr gut erkennbar. Lediglich das Aluminium ist bereits - wie in den anderen drei Proben auch - homogen in der Schicht verteilt. Erhöht man 
die Temperatur auf $650{ }^{\circ} \mathrm{C}$ (Abb. 7.4 (b)), werden der Anstieg des Sauerstoff- bzw. das Abfallen des Stickstoff- und des Titanprofils flacher. Außerdem fällt die Konzentration des Sauerstoffs an der Schichtoberfläche nicht mehr auf null ab, sondern nähert sich einem endlichen Wert von circa 5 at.-\%. Ein ähnliches Bild ergibt sich, wenn bei $450{ }^{\circ} \mathrm{C}$ die Beschleunigungsspannung auf $1000 \mathrm{~V}$ erhöht wird (Abb. 7.4 (c)). Auch hier werden die Konzentrationskurven flacher, und die Sauerstoffkonzentration an der Schichtoberfläche nähert sich etwa 5 at.-\% an. Wird nun bei der hohen kinetischen Energie des Stickstoffionenstrahls auch noch die Substrattemperatur auf $650{ }^{\circ} \mathrm{C}$ erhöht (Abb. 7.4 (d)), ändert sich die From der Konzentrationsprofile erheblich. Sauerstoffanstieg und Stickstoffabnahme treten nun erst an der Grenzschicht zwischen Substrat und dünner Schicht auf, was aber eher durch die oxidische Zusammensetzung des MgO-Substrates erklärt werden kann. In den übrigen Bereichen der Probe sind sowohl Stickstoff als auch Sauerstoff relativ gleichmäßig verteilt, wobei die Konzentrationen beider Elemente zu größeren Schichttiefen hin leicht zunehmen. Die Verteilung des Titans bleibt allerdings auch im letzten Fall stark inhomogen. An der Probenoberfläche ist sie dabei beinahe doppelt so hoch wie in der Nähe der Substratoberfläche.

Die Betrachtung dieser Ergebnisse legt den Schluss nahe, dass die Zusammensetzung der Schichten stark durch die Bildung verschiedener oxidischer bzw. nitridischer Verbindungen und ihren Einfluss auf Diffusionsprozesse bestimmt wird. Sind Substrattemperatur und/oder kinetische Energie der Stickstoffionen gering, wird bei der Deposition zunächst offensichtlich mehr Sauerstoff als Stickstoff in die Schichten eingebaut. Im weiteren Verlauf des Schichtwachstums wird zunehmend mehr Stickstoff im Film inkorporiert, was gleichzeitig eine deutlich Reduzierung der Sauerstoffaufnahme bewirkt. So fallen - in Richtung des Filmwachstums gesehen - Stickstoffanstieg und Sauerstoffabfall immer zusammen. Bei der $1000 \mathrm{~V} / 650{ }^{\circ} \mathrm{C}-$ Probe wird von Wachstumsbeginn an viel Stickstoff aufgenommen und der Sauerstoffgehalt liegt von Anfang an bei vergleichsweise geringen Werten von etwa 10 at.-\%. Im Zusammenhang mit dem hohen Sauerstoffgehalt der Proben in der Nähe des Substrates kann man eine weitere Feststellung treffen. Und zwar diffundiert Magnesium in beinahe allen Fällen relativ weit (50-100 nm) in die Schicht hinein. Lediglich in einem Fall - der bei $650 \mathrm{C}$ mit $1000 \mathrm{~V}$ deponierten Probe - ist dies nicht der Fall. Gleichzeitig ist auch in den Proben mit der stärkeren Mg-Diffusion der Sauerstoffgehalt in tieferen Schichtbereichen höher als in der Probe mit geringerer Mg-Diffusion. Unter Umständen kann also auch eine stärkere Vermischung dem MgO-Substrat zum Teil für die erhöhte Sauerstoffkonzentration verantwortlich sein. Im Falle der letztgenannten Probe könnte es aufgrund der hohen Temperatur und der gleichzeitig hohen kinetischen Energie der Stickstoffionen zur sehr schnellen Bildung einer stickstoffhaltigen Verbindung kommen, die die Diffusion aus dem Substrat in die Schicht unterdrückt. Kubisches Titannitrid TiN käme dafür in Frage, da sich sein Gitterparameter $a=0,424 \mathrm{~nm}$ nur wenig von dem des kubischen MgO-Substrats $a=0,421 \mathrm{~nm}$ unterscheidet, was eine Bildung des 

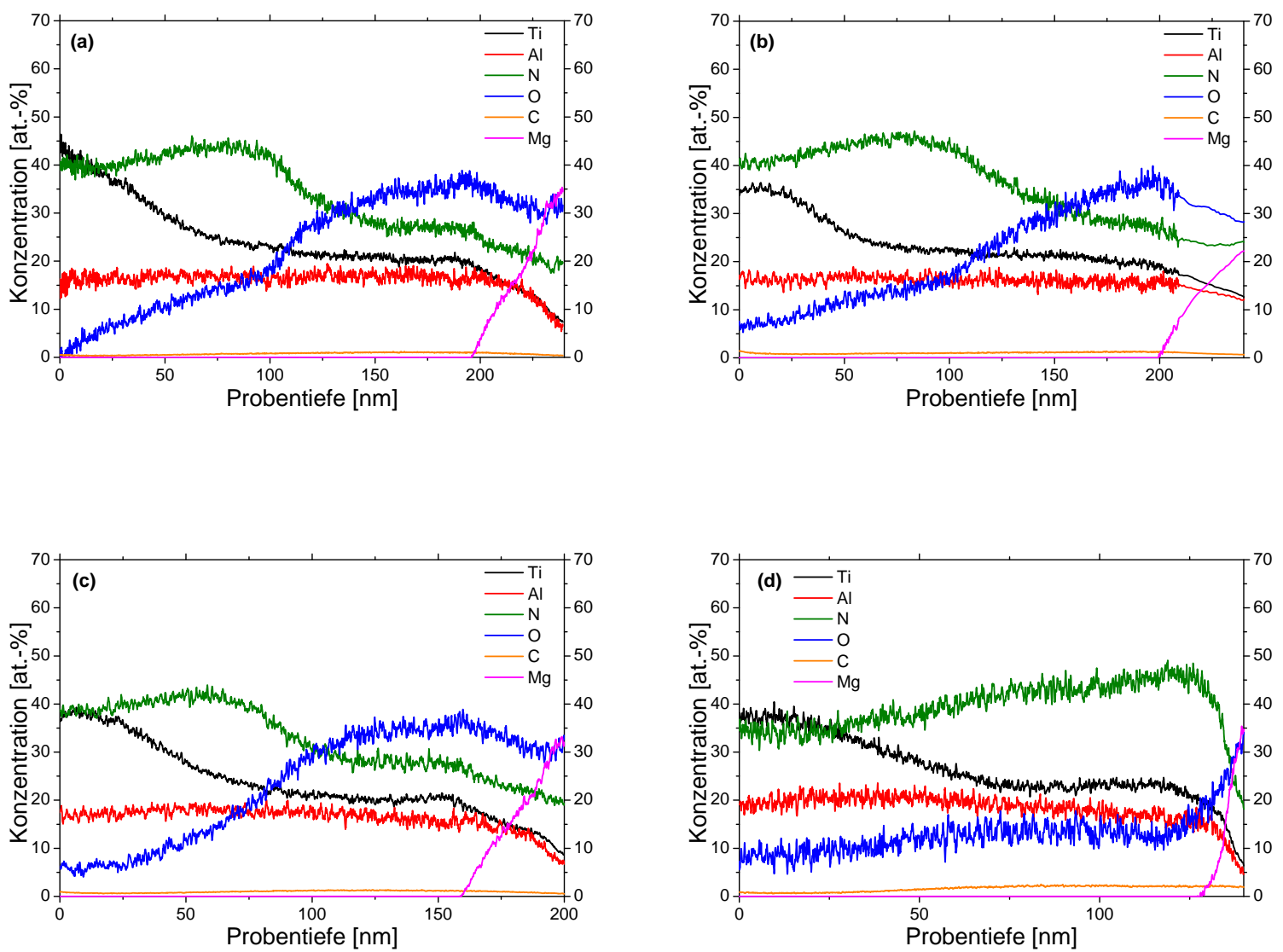

Abbildung 7.4: GDOES-Profile von Ti/Al/N-Schichten bei 200 und $1000 \mathrm{~V}$ Beschleunigungsspannung. (a): $200 \mathrm{~V}, 450{ }^{\circ} \mathrm{C}$; (b): $200 \mathrm{~V}, 650^{\circ} \mathrm{C}$; (c): $1000 \mathrm{~V}, 450{ }^{\circ} \mathrm{C}$; (d): $1000 \mathrm{~V}, 650{ }^{\circ} \mathrm{C}$. Substrattemperatur und kinetische Energie der eingestrahlten Ionen haben einen deutlichen Einfluss auf die Konzentrationsprofile der einzelnen Elemente. Durch eine Umschaltung in der Messwertaufnahme des Gerätes werden die Kurven in Teilbild (b) zum Ende hin geglättet.

TiN begünstigen würde. Trotz der beschriebenen Probleme zeigt sich aber ein Vorteil bei der Verwendung des Stickstoff-Ionenstrahls. Es ist gelungen, nitridhaltige Schichten bei Hintergrunddrücken von etwa $p=10^{-2} \mathrm{~Pa}$ zu deponieren, die etwa eine Größenordnung unterhalb der Drücke bei der konventionellen reaktiven PLD ( $\left.p=10^{-1} \mathrm{~Pa}\right)$ lagen. Bei vergleichbaren Drücken von $p=10^{-2} \mathrm{~Pa}$ wurden bei der reaktiven PLD ohne Ionenstrahl nur Spuren von TiN in den Schichten beobachtet [102]. Auch in den hier vorgestellten Experimenten konnte durch das Anlegen der Beschleunigungsspannung der Stickstoffanteil in den Filmen deutlich erhöht werden.

Aufgrund der Tatsache, dass alle Schichten röntgenamorph sind, lässt sich die Bildung chemischer Phasen in den Schichten nicht nachvollziehen. Die oben aufgestellten Hypothesen sind damit zunächst nicht überprüfbar. Aus den GDOES-Messungen ist jedoch ersichtlich, dass in tieferen Schichtbereichen die Summe der Stickstoff- und Sauerstoffkon- 
zentration die Summe der Titan- und Aluminiumkonzentration übersteigt: $c(\mathrm{~N})+\mathrm{c}(\mathrm{O})>$ $\mathrm{c}(\mathrm{Ti})+\mathrm{c}(\mathrm{Al})$. Es müssen also Verbindungen der Form $\mathrm{Me}_{x}(\mathrm{O}, \mathrm{N})_{y}(\mathrm{Me}=$ Ti oder $\mathrm{Al})$ mit $y>x$ vorliegen. Bei den stabilen Nitriden von Titan und Aluminium handelt es sich jedoch um 1:1-Verbindungen TiN bzw. AlN [59]. Es existieren jedoch die sauerstoffreichen Oxdide $\mathrm{TiO}_{x}(x \leq 2)$ und $\mathrm{Al}_{2} \mathrm{O}_{3}$, sowie zusätzlich so genannte Titanoxynitride der Form $\mathrm{TiN}_{x} \mathrm{O}_{y}$, in denen $x+y>1$ ist [160]. Zu den Schichtoberflächen hin erhöht sich aber der Gehalt an Titan, und es kommt offensichtlich zur Bildung von Titannitrid, was sich an der goldenen Färbung der Proben erkennen lässt. Diese vermutliche Bildung zahlreicher Verbindungen mit relativ großen Homogenitätsbereichen und die oben erläuterten Diffusionsprozesse verhindern offensichtlich die Bildung größerer kristalliner Bereiche in den Schichten, die einer Röntgenbeugungsmessung zugänglich wären. 


\section{Zusammenfassung und Ausblick}

Die Ziele der in dieser Dissertation vorgestellten Arbeiten waren der Aufbau einer experimentellen Anlage zur Pulslaserdeposition und erste Untersuchungen zur Synthese von dünnen Schichten aus MAX-Phasen. Der Aufbau des Experiments war erfolgreich. Dünne Schichten guter makroskopischer Qualität konnten mittels PLD in einem großen Temperaturbereich hergestellt werden, wobei durch die Benutzung der zur Verfügung stehenden Ionenquelle der Einfluss einer Ionenbestrahlung der Schicht während der Deposition untersucht und nachgewiesen werden konnte. Die Untersuchungen zu den MAX-Schichten gliederten sich in zwei Teile. Dies war zum einen die PLD von bereits synthetisierten MAX-Phasen-Targets der Zusammensetzungen $\mathrm{Ti}_{3} \mathrm{SiC}_{2}$ und $\mathrm{Cr}_{2} \mathrm{AlC}$ und zum anderen die PLD der reinen Metalle Titan und Aluminium mit einem auf das Substrat gerichteten Stickstoffionenstrahl, um die nitridische MAX-Phase reaktiv während des Schichtwachstums zu bilden.

Bei der Deposition aus den MAX-Phasen-Targets konnte in keinem Fall die stöchiometrische Zusammensetzung der Targets in den Filmen erhalten werden. Statt dessen kam es zum Verlust der A- und X-Elemente. Im Fall von $\mathrm{Ti}_{3} \mathrm{SiC}_{2}$ also von Silizium und Kohlenstoff, bzw. bei Ablation des $\mathrm{Cr}_{2}$ AlC-Targets zum Verlust von Aluminium und Kohlenstoff. Offensichtlich werden die verschiedenen Elemente durch die kinetische Energie der ankommenden Teilchen unterschiedlich stark wieder aus der Schicht abgesputtert. Die Bildung unterschiedlicher Phasen trägt dabei zu einer mehr oder weniger guten Haftung der Elemente in den Schichten bei, wie die durch die Formation einer festen Lösung von Aluminium in Chrom hervorgerufenen Aluminiuminhomogenitäten bei der Deposition von $\mathrm{Cr}_{2} \mathrm{AlC}$ zeigen. Bei der Ablation von $\mathrm{Ti}_{3} \mathrm{SiC}_{2}$ tritt - durch die höhere Sauerstoffaffinität von Titan und Silizium - zusätzlich noch eine starke Aufnahme von Sauerstoff auf, die zur Bildung von flüchtigem Siliziummonoxid führt. Da die MAX-Verbindungen sich aber nur entsprechend der Summenformel $\mathrm{M}_{n+1} \mathrm{AX}_{n}$ bilden, wurde ihre Bildung in den Schichten folglich nicht beobachtet. Entsprechend der engen strukturellen Verwandschaft von MAX-Phasen mit ihren binären MX-Verbindungen kam es statt dessen zur Bildung von Titancarbid TiC, bzw. zweier Chromcarbide $\mathrm{Cr}_{7} \mathrm{C}_{3}$ und $\mathrm{Cr}_{23} \mathrm{C}_{6}$. Größere kristalline Bereiche treten dabei erst bei Substrattemperaturen $\geq 450{ }^{\circ} \mathrm{C}$ auf, wobei mit steigender Temperatur die Größe der Kristallite zunimmt. Auch das gewählte Substratmaterial hat einen Einfluss auf die Formation der kristallinen Phasen. Einerseits kam es bei der 
Verwendung von einkristallinem $\mathrm{MgO}(100)$ zum epitaktischen Wachstum von $\mathrm{Cr}_{2} \mathrm{Al}$. Andererseits behinderten Diffusionsprozesse auf dem rauhen und inhomogenen Edelstahlsubstrat die Bildung kristalliner Bereiche erheblich. Die A-Elemente Silizium und Aluminium sind amorph in der Schicht verteilt und bilden keine durch Röntgenbeugung nachweisbare Phasen. Im Falle von $\mathrm{Ti}_{3} \mathrm{SiC}_{2}$ konnte aber durch XPS-Messungen gezeigt werden, dass das Silizium sowohl elementar als auch als Siliziumcarbid bzw. -oxid in den Proben vorliegt.

In ihren physikalischen Eigenschaften wie Schichthärte und elektrische Leitfähigkeit sind die $\mathrm{Cr} / \mathrm{Al} / \mathrm{C}-$ Schichten vergleichbar mit Filmen, die ausschließlich die entsprechenden Carbide enthalten. Die $\mathrm{Ti}_{3} \mathrm{SiC}_{2}$-Proben hingegen sind sowohl weicher als auch weniger gut elektrisch leitend als reine Titancarbidschichten, was sich vor allem durch den hohen Sauerstoffgehalt in den Proben erklären lässt.

Die Verwendung des chemisch inerten Argonionenstrahls beeinflusste die Eigenschaften der Schichten ebenfalls. So waren die bestrahlten Proben häufig härter als die unbestrahlten. Des Weiteren bewirkte bei der Deposition von $\mathrm{Ti}_{3} \mathrm{SiC}_{2}$ auf Edelstahl der Ionenbeschuss eine erhebliche Verstärkung der Interdiffusion von Elementen zwischen Substrat und Schicht. Bei der höchsten Substrattemperatur von $650{ }^{\circ} \mathrm{C}$ war bei Zuschaltung des Ionenstrahls eine Deposition von $\mathrm{Ti}_{3} \mathrm{SiC}_{2}$ auf einkristallinem $\mathrm{Si}(100)$ nicht mehr möglich, da die Schicht bereits während der Abscheidung riss und abblätterte. Verglichen mit den in Abschnitt 2.2.4 beschriebenen Möglichkeiten der ionenstrahlunterstützten PLD war der Einfluss allerdings relativ gering. Dies lässt sich mit der vergleichsweise großen Divergenz des Strahls aus der Ionenquelle (ca. $15^{\circ}$ ) erklären.

Bei der reaktiven PLD von Titan und Aluminium mit zugeschaltetem Stickstoffionenstrahl kam es ebenfalls zum Auftreten erheblicher Probleme. Zunächst konnte wiederum nicht das im Target zur Bildung von $\mathrm{Ti}_{2} \mathrm{AlN}$ vorgelegte Verhältnis $\mathrm{Ti}: \mathrm{Al}=2: 1$ in die Schichten übertragen werden. Schon bei den Depositionen ohne Beschleunigungsspannung lag es bei Ti:Al =1,8:1, und bei angelegter Spannung verringerte sich der Titangehalt im Verhältnis zum Aluminium weiter. Zusätzlich kam es durch die mangelnde Reinheit des verwendeten Stickstoffgases zum massiven unerwünschten Einbau von Sauerstoff in die Schichten. Dadurch entstand vermutlich eine Vielzahl von oxidischen und nitridischen Verbindungen in den Filmen, in denen außerdem noch starke Diffusionsvorgänge stattfanden. Diese beiden Effekte verhinderten die Bildung messbarer kristalliner Bereiche in den Schichten, die für eine genauere Untersuchung der Vorgänge während des Schichtwachstums notwendig gewesen wären.

Die größte Herausforderung für weitere Experimente mit MAX-Phasen stellt also die Kontrolle der Stöchiometrie dar. Im Rahmen dieser Arbeit wurde hinlänglich gezeigt, dass die benötigte $\mathrm{M}_{n+1} \mathrm{AX}_{n}$-Zusammensetzung nicht durch die PLD aus den MAX-PhasenTargets erreicht werden kann. Die Lösung dieses Problems ist in der Ablation von Targets aus den reinen Elementen M, A und X zu suchen, wobei durch die computergesteuerte 
Kopplung des Targethaltermotors mit der Lasersteuerung - bei richtiger Wahl der Anzahl Laserpulse pro Target - jeweils die erforderlichen Mengen der einzelnen Bestandteile in Zyklen deponiert werden können. Ein weiteres Problem ist die teilweise auftretende Inkorporation von Sauerstoff. Bei der Ablation von Titan und Silizium ist der in der Kammer herrschende Vakuumdruck von etwa $10^{-5} \mathrm{~Pa}\left(=10^{-7} \mathrm{mbar}\right)$ noch zu hoch, so dass die Oxidation der Elemente auftritt. Die Erweiterung um eine weitere Pumpstufe (Getteroder Kryopumpe) ist folglich notwendig, um die Sauerstoffaufnahme zu unterdrücken. Ähnliches gilt für die reaktive Pulslaserdeposition. Auch hier ist die Verwendung eines einzigen Targets mit einem festen Verhältnis der Elemente $M$ und A nicht zielführend, da sich das M:A-Verhältnis in der Schicht mit der angelegten Beschleunigungsspannung ändert. Eine große Rolle spielt offensichtlich auch die Reinheit der verwendeten reaktiven Gases. Auch in Fall der geringsten Sauerstoffverunreinigung bei der reaktiven PLD wurde mit dem hier verwendeten Stickstoff 5.0-Gas (99,999 \%) noch eine mittlere Sauerstoffkonzentration von etwa 12 at.- $\%$ gemessen. Hier bietet sich eine entsprechende Aufbereitung des Gases (z.B. Trocknung) vor dem Einleiten in die Kammer an.

Schließlich ist es für die Anwendung des Ionenstrahls - sowohl inert als auch reaktiv zur Beeinflussung des kristallinen Schichtwachstums wichtig, die Form des Strahls selbst besser zu kontrollieren und durch eine geeignete Fokussierung vor allem die Divergenz des Strahls zu verringern.

Die in dieser Arbeit vorgelegten Ergebnisse zeigen also die Schwierigkeiten und Probleme, die mit der Pulslaserdeposition von MAX-Phasen verbunden sind. Gleichzeitig liefert sie aber wichtige Anhaltspunkte für eine gezielte Verbesserung des experimentellen Ansatzes, um das ursprüngliche Ziel, die erfolgreiche Herstellung von dünnen MAX-Schichten mittels PLD, erreichen zu können. 


\section{Abbildungsverzeichnis}

2.1 Gruppen von MAX-Phasen . . . . . . . . . . . . . . . . . . . . 4

2.2 Stapelfolgen in MAX-Phasen . . . . . . . . . . . . . . . . . . 4

2.3 Phasenumwandlung von ternären $\mathrm{M}_{n+1} \mathrm{AX}_{n}$-Verbindungen . . . . . . . . 5

2.4 Verhalten von MAX-Phasen unter mechanischer Belastung . . . . . . . . . 7

2.5 Ternäres Phasendiagramm des Systems Ti-Si-C bei $1250{ }^{\circ} \mathrm{C} \quad \ldots . . .9$

2.6 Kristallstrukturen von $\mathrm{Ti}_{3} \mathrm{SiC}_{2}$ und $\mathrm{TiC} \ldots \ldots \ldots$. . . . . . . . 10

2.7 Ti-Si-C-Schicht mit zufälliger Stapelfolge . . . . . . . . . . . . . . . . 12

2.8 Thermische Zersetzung von $\mathrm{Ti}_{3} \mathrm{SiC}_{2}$-Schichten ab $1200{ }^{\circ} \mathrm{C} \ldots \ldots$. . . . . . . 12

2.9 Wachstum einer $\mathrm{Ti}_{3} \mathrm{SiC}_{2}$-Schicht auf einer $\mathrm{TiC}_{x}$-Pufferschicht . . . . . . . . 13

2.10 Kontaktwiderstand einer $n c-\mathrm{TiC} / a$-SiC-Schicht . . . . . . . . . . . . . . . 14

2.11 Ternäres Phasendiagramm des Systems Cr-Al-C bei $1000{ }^{\circ} \mathrm{C}$. . . . . . . 15

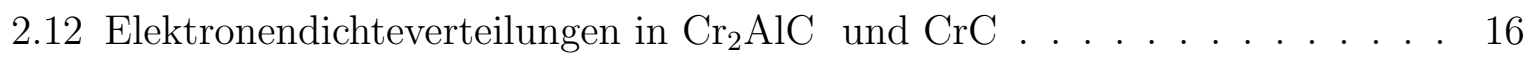

2.13 Isothermale Schnitte durch das Ti-Al-N-Phasendiagramm bei $1325^{\circ} \mathrm{C}$ und $900{ }^{\circ} \mathrm{C} \ldots \ldots \ldots \ldots \ldots \ldots$

2.14 Thermische Zersetzung von $\mathrm{Ti}_{2} \mathrm{AlN}_{1-x}$-Schichten ab $800{ }^{\circ} \mathrm{C}$. . . . . . . . 20

2.15 Modell des Schichtwachstums von $\mathrm{Ti}_{2} \mathrm{AlN}_{1-x}$ auf $\mathrm{MgO}(111) \quad \ldots . . . . .21$

2.16 Schematische Zeichnung einer PLD-Anlage . . . . . . . . . . . . . . . 23

2.17 Droplet in einer TiC/Si-PLD-Schicht . . . . . . . . . . . . . . . 24

2.18 Oberfläche eines $\mathrm{ZrO}_{2}$-Targets nach Laserablation . . . . . . . . . . . . 26

2.19 Schematische Darstellung einer cross-beam PLD-Anlage . . . . . . . . . . . 27

2.20 Verbesserung der Schichtmorphologie bei der PLD . . . . . . . . . . . . . 27

2.21 Winkelabhängigkeiten von Abscheidungsrate und maximaler kinetischer Energie der Ionen bei der PLD . . . . . . . . . . . . . . . . . . . 29

2.22 Optische Emission von $\mathrm{CN}$ - und $\mathrm{C}_{2}$-entlang der Plasmaachse . . . . . . . 30

2.23 IBA-PLD von hexagonalem und kubischem Bornitrid . . . . . . . . . . . . 33

2.24 Verzerrung der Einheitszelle von AlN durch Ionenbestrahlung bei der IBA-

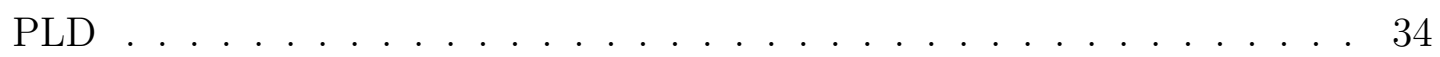

3.1 Strahlengeometrie bei der $\theta / 2 \theta$-Röntgendiffraktometrie . . . . . . . . . 37

3.2 Strahlengeometrie bei streifendem Einfall . . . . . . . . . . . . . . 37 
3.3 Prinzipieller Aufbau der GDOES-Hohlanodenquelle. . . . . . . . . . . . . . 38

3.4 Messoptik eines GDOES-Messgerätes. . . . . . . . . . . . . . . . . . . 40

3.5 Elektronenmikroskopische Aufnahme eines GDOES-Messflecks . . . . . . . 42

3.6 Geräteaufbau zur Photoelektronenspektroskopie . . . . . . . . . . . . . . . 44

3.7 Mittlere freie Weglängen von Elektronen in Metallen . . . . . . . . . . . . 44

3.8 Mikrohärtemessung . . . . . . . . . . . . . . . . . . . . . . 45

3.9 Bestimmung des Schichtwiderstands $R_{s} \ldots \ldots \ldots \ldots$. . . . . 48

4.1 Konstruktionszeichnung der PLD-Kammer . . . . . . . . . . . . . . . 50

4.2 CAD-Zeichnung des Probenaufnahmeflansches . . . . . . . . . . . . . . . 51

4.3 Foto der Probenaufnahme in der PLD-Kammer . . . . . . . . . . . . . . 52

4.4 Konstruktionszeichnung des Substrathalters . . . . . . . . . . . . . 53

4.5 Boralectric ${ }^{\circledR}{ }_{-}$Heizelement. . . . . . . . . . . . . . . . . . . . . 53

4.6 Foto der Ionenquelle . . . . . . . . . . . . . . . . . . . . . 54

4.7 Ionenquelle und Probenhalter im eingebauten Zustand . . . . . . . . . . 55

4.8 Schaltplan der Pumpen und Ventile . . . . . . . . . . . . . . . . . 56

4.9 Konstruktionszeichnung des Targethalters . . . . . . . . . . . . . . . 58

4.10 Foto der gesamten PLD-Anlage . . . . . . . . . . . . . . . . . 59

4.11 Laser, Strahlengang und Fokussierlinse der PLD-Anlage . . . . . . . . . . . 60

5.1 Schematische Darstellung der Probenherstellung . . . . . . . . . . . . . . 62

5.2 Charakterisierung des $\mathrm{Ti}_{3} \mathrm{SiC}_{2}$-Ablationstargets . . . . . . . . . 63

5.3 Charakterisierung des $\mathrm{Cr}_{2} \mathrm{AlC}$-Ablationstargets . . . . . . . . . . . 64

6.1 REM-Aufnahme des Querschnitts durch eine $\mathrm{Ti}_{3} \mathrm{SiC}_{2}$-Schicht . . . . . . . 66

6.2 Zusammensetzungen aller vom $\mathrm{Ti}_{3} \mathrm{SiC}_{2}$-Target auf $\mathrm{MgO}$ deponierten Schich-

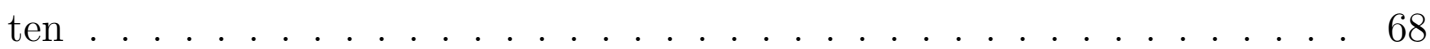

6.3 GDOES-Messungen zweier auf $\mathrm{MgO}(100)$ deponierter Ti/Si/C-Schichten . 69

6.4 Röntgendiffraktogramme der Proben auf $\mathrm{MgO}(100)$ ohne Ionenstrahl . . . 71

6.5 Röntgendiffraktogramme der Proben auf $\mathrm{MgO}(100)$ mit Ionenstrahl . . . . 72

6.6 HR-TEM-Aufnahme einer Ti/Si/C-Schicht. . . . . . . . . . . . . . . . 73

6.7 TEM-SAED-Messung einer Ti/Si/C-Schicht . . . . . . . . . . . . . . . 74

6.8 Schichthärten und Sauerstoffgehalt der auf $\mathrm{MgO}(100)$ deponierten Proben . 75

6.9 Zusammensetzungen aller vom $\mathrm{Ti}_{3} \mathrm{SiC}_{2}$-Target auf $\mathrm{Si}$ deponierten Schichten 79

6.10 GDOES-Messungen zweier auf $\mathrm{Si}(100)$ deponierter Ti/Si/C-Schichten . . . 79

6.11 Röntgendiffraktogramme der Proben auf Si(100) ohne Ionenstrahl . . . . . 80

6.12 Röntgendiffraktogramme der Proben auf Si(100) mit Ionenstrahl . . . . . . 81

6.13 Schichthärten und Sauerstoffgehalt der auf $\operatorname{Si}(100)$ deponierten Proben . . 82

6.14 XPS-Messung des Ti 2p-Orbitals einer Ti/Si/C-Schicht . . . . . . . . . . . 84

6.15 XPS-Messung des Si 2p-Orbitals einer Ti/Si/C-Schicht . . . . . . . . . . . 84 
6.16 XPS-Messung des C 1s-Orbitals einer Ti/Si/C-Schicht . . . . . . . . . . . 85

6.17 Leitfähigkeiten der auf $\mathrm{MgO}$ und $\mathrm{Si}$ deponierten $\mathrm{Ti} / \mathrm{Si} / \mathrm{C}-\mathrm{Schichten}$. . . . 86

6.18 Zusammensetzungen aller vom $\mathrm{Ti}_{3} \mathrm{SiC}_{2}$-Target auf Edelstahl deponierten Schichten . . . . . . . . . . . . . . . . . . . 88

6.19 GDOES-Messungen zweier auf Edelstahl deponierter Ti/Si/C-Schichten . . 89

6.20 Röntgendiffraktogramme der Proben auf Edelstahl ohne Ionenstrahl . . . 90

6.21 Röntgendiffraktogramme der Proben auf Edelstahl mit Ionenstrahl . . . . . 91

6.22 Schichthärten der auf Edelstahl deponierten Proben . . . . . . . . . . . . . 92

6.23 REM-Aufnahme des Querschnitts durch eine $\mathrm{Cr}_{2} \mathrm{AlC}-\mathrm{Schich}$. . . . . . . . 96

6.24 Zusammensetzungen aller vom $\mathrm{Cr}_{2} \mathrm{AlC}$-Target auf $\mathrm{MgO}$ deponierten Schich-

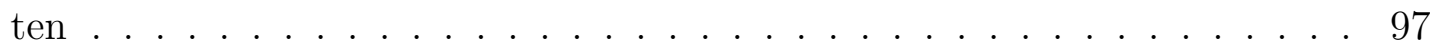

6.25 GDOES-Messungen zweier bei Raumtemperatur auf $\mathrm{MgO}(100)$ deponierter

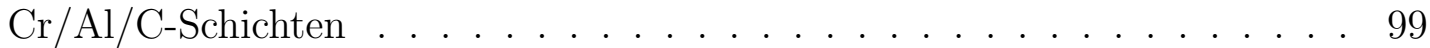

6.26 GDOES-Messungen zweier bei $650^{\circ} \mathrm{C}$ auf $\mathrm{MgO}(100)$ deponierter $\mathrm{Cr} / \mathrm{Al} / \mathrm{C}$ Schichten . . . . . . . . . . . . . . . . . 99

6.27 Röntgendiffraktogramme der $\mathrm{Cr}_{2} \mathrm{AlC}$-Proben auf $\mathrm{MgO}$ ohne Ionenstrahl . . 101

6.28 Röntgendiffraktogramme der $\mathrm{Cr}_{2} \mathrm{AlC}$-Proben auf $\mathrm{MgO}$ mit Ionenstrahl . 101

6.29 Zusammensetzung der kristallinen MgO-Proben im Phasendiagramm . . . 102

6.30 HR-TEM-Aufnahme des Übergangsbereiches zwischen MgO-Substrat und $\mathrm{Cr} / \mathrm{Al} / \mathrm{C}-\mathrm{Film} \ldots \ldots \ldots$. . . . . . . . . . . . . . . . . . . . . . . . . . . .

6.31 Härtemessungen der auf $\mathrm{MgO}$ deponierten $\mathrm{Cr}_{2} \mathrm{AlC}$-Proben . . . . . . . . . 105

6.32 Zusammensetzungen aller vom $\mathrm{Cr}_{2} \mathrm{AlC}$-Target auf $\mathrm{SiO}_{2}$ deponierten Schich-

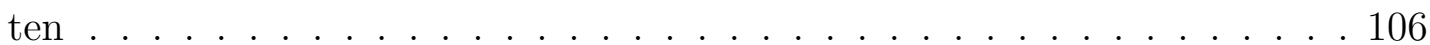

6.33 GDOES-Messungen zweier bei Raumtemperatur auf $\mathrm{SiO}_{2}$ deponierter $\mathrm{Cr} / \mathrm{Al} / \mathrm{C}$ Schichten . . . . . . . . . . . . . . . . . 107

6.34 GDOES-Messungen zweier bei Raumtemperatur auf $\mathrm{MgO}(100)$ deponierter $\mathrm{Cr} / \mathrm{Al} / \mathrm{C}-\mathrm{Schichten} \mathrm{\ldots} \mathrm{.} \mathrm{.} \mathrm{.} \mathrm{.} \mathrm{.} \mathrm{.} \mathrm{.} \mathrm{.} \mathrm{.} \mathrm{.} \mathrm{.} \mathrm{.} \mathrm{.} \mathrm{.} \mathrm{.} \mathrm{.} \mathrm{.} 108$

6.35 Röntgendiffraktogramme der $\mathrm{Cr}_{2} \mathrm{AlC}-\mathrm{Proben}$ auf $\mathrm{SiO}_{2}$ ohne Ionenstrahl . . 108

6.36 Röntgendiffraktogramme der $\mathrm{Cr}_{2} \mathrm{AlC}-\mathrm{Proben}$ auf $\mathrm{SiO}_{2}$ mit Ionenstrahl . . . 109

6.37 Härtemessungen der auf $\mathrm{SiO}_{2}$ deponierten $\mathrm{Cr}_{2} \mathrm{AlC}$-Proben . . . . . . . . . 110

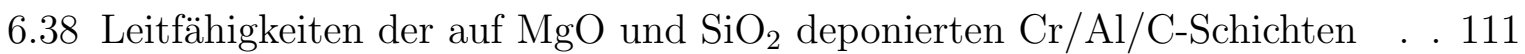

6.39 Zusammensetzungen aller vom $\mathrm{Cr}_{2} \mathrm{AlC}$-Target auf Edelstahl deponierten Schichten . . . . . . . . . . . . . . . . . . . 112

6.40 Vergleich der Eisendiffusion in $\mathrm{Cr} / \mathrm{Al} / \mathrm{C}-$ und Ti/Si/C-Schichten . . . . . . 113

6.41 Röntgendiffraktogramme der $\mathrm{Cr}_{2} \mathrm{AlC}$-Proben auf Edelstahl ohne Ionenstrahl114

6.42 Röntgendiffraktogramme der $\mathrm{Cr}_{2} \mathrm{AlC}$-Proben auf Edelstahl mit Ionenstrahl 115

6.43 Vergleich der Elementarzellen von $\mathrm{Cr}_{7} \mathrm{C}_{3}$ und $\mathrm{Cr}_{23} \mathrm{C}_{6} \ldots \ldots$. . . . . . . 115

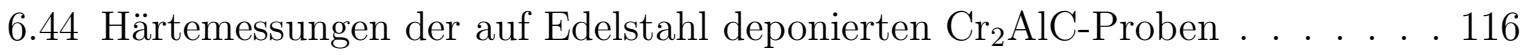


7.1 Zusammensetzungen der Ti-Al-N-Schichten bei $450{ }^{\circ} \mathrm{C}$. . . . . . . . . . . 121

7.2 Zusammensetzungen der Ti-Al-N-Schichten bei $650{ }^{\circ} \mathrm{C}$. . . . . . . . . . . 122

7.3 GDOES-Messungen zweier ohne Beschleunigungsspannung auf $\mathrm{MgO}(100)$ deponierter $\mathrm{Ti} / \mathrm{Al} / \mathrm{N}-\mathrm{Schichten}$. . . . . . . . . . . . . . . . . . . 122

7.4 GDOES-Profile von Ti/Al/N-Schichten bei 200 und $1000 \mathrm{~V}$ Beschleunigungsspannung . . . . . . . . . . . . . . . . . . . . 124 


\section{Tabellenverzeichnis}

2.1 Raumtemperaturleitfähigkeiten von MAX-Phasen . . . . . . . . . . . 6

2.2 Eigenschaften von $\mathrm{Ti}_{2} \mathrm{AlN}_{1-x}$ und $\mathrm{Ti}_{4} \mathrm{AlN}_{3-x} \ldots \ldots \ldots \ldots$

2.3 Mit reaktiver PLD hergestellte Verbindungen . . . . . . . . . . . . . . . . 29

2.4 Mit ionenstrahlunterstützter PLD hergestellte Schichten . . . . . . . . . . 32

5.1 Übersicht aller Ti-Si-C-Proben . . . . . . . . . . . . . . . . . . . . . 65

5.2 Übersicht aller Cr-Al-C-Proben . . . . . . . . . . . . . . . . . . . 65

5.3 Übersicht aller Ti-Al-N-Proben . . . . . . . . . . . . . . . . . . 65

6.1 Netzebenenabstände aus der TEM-FFT-Rechnung . . . . . . . . . . . . 73 


\section{Literaturverzeichnis}

[1] S. Somiya, F. Aldinger, R.M. Spriggs, K. Uchino, K Koumoto, and M. Kaneno, editors. Handbook of Advanced Ceramics Vol. II, Processing and their applications. Academic Press, 2003.

[2] M. W. Barsoum and T. El-Raghy. The MAX phases: Unique new carbide and nitride materials. American Scientist, 89(4):334-343, 2001.

[3] M. W. Barsoum, T. Zhen, A. Zhou, S. Basu, and S. R. Kalidindi. Microscale modeling of kinking nonlinear elastic solids. Physical Review B, 71(13):134101, 2005 .

[4] M. W. Barsoum. The M(n+1)AX(n) phases: A new class of solids; Thermodynamically stable nanolaminates. Progress in Solid State Chemistry, 28(1-4):201-281, 2000 .

[5] W. Jeitschko, H. Nowotny, and F. Benesovsky. Ti2AlN, Eine stickstoffhaltige HPhase. Monatshefte für Chemie, 94(6):1198-1200, 1963.

[6] W. Jeitschko, H. Nowotny, and F. Benesovsky. Kohlenstoffhaltige ternäre Verbindungen (H-Phase). Monatshefte für Chemie, 94(4):672-676, 1963.

[7] W. Jeitschko, H. Nowotny, and F. Benesovsky. Die H-Phasen Ti2InC, Zr2InC, Hf2InC und Ti2GeC. Monatshefte für Chemie, 94(6):1201-1205, 1963.

[8] W Jeitschko and H. Nowotny. Die Kristallstruktur von Ti3SiC2 - ein neuer Komplexcarbid-Typ. Monatshefte für Chemie, 98(2):329-337, 1967.

[9] H. Nowotny. Strukturchemie einiger Verbindungen der Übergangsmetalle mit den Elementen C, Si, Ge, Sn. Progress in Solid State Chemistry, 5:27-70, 1971.

[10] M. W. Barsoum and T. El-Raghy. Synthesis and characterization of a remarkable ceramic: Ti3SiC2. Journal of the American Ceramic Society, 79(7):1953-1956, 1996. 
[11] A.H. Cottrell. Chemical bonding in transition metal carbides (Institute of Materials Book, 613). Ashgate Publishing, 1995.

[12] E. H. Kisi, J. A. A. Crossley, S. Myhra, and M. W. Barsoum. Structure and crystal chemistry of Ti3SiC2. Journal Of Physics And Chemistry Of Solids, 59(9):1437$1443,1998$.

[13] Y. Zhou and Z. Sun. Electronic structure and bonding properties in layered ternary carbide Ti3SiC2. Journal of Physics: Condensed Matter, 12:L457-L462, 2000.

[14] N. Haddad, E. Garcia-Caurel, L. Hultman, M. W. Barsoum, and G. Hug. Dielectric properties of Ti2AlC and Ti2AlN MAX phases: The conductivity anisotropy. Journal Of Applied Physics, 104(2):023531, 2008.

[15] M. W. Barsoum, H. I. Yoo, I. K. Polushina, V. Y. Rud, Y. V. Rud, and T. ElRaghy. Electrical conductivity, thermopower, and Hall effect of Ti3AIC2, Ti4AIN3, and Ti3SiC2. Physical Review B, 62(15):10194-10198, 2000.

[16] W. S. Williams. Scattering of electrons by vacancies in nonstoichiometric crystals of titanium carbide. Physical Review A-General Physics, 135(2A):A505-A510, 1964.

[17] D.R. Lide, editor. CRC Handbook of Chemistry and Physics. CRC Press, 2002.

[18] J. J. Nickl, K.K. Schweitzer, and P. Luxenberg. Gasphasenabscheidung im System Ti-Si-C. Journal Of The Less-Common Metals, 26(3):335, 1972.

[19] R. Pampuch, J. Lis, L. Stobierski, and M. Tymkiewicz. Solid combustion synthesis of Ti3SiC2. Journal of the European Ceramic Society, 5(5):283-287, 1989.

[20] M. W. Barsoum, M. Ali, and T. El-Raghy. Processing and characterization of Ti2AlC, Ti2AlN, and Ti2AlC0.5N0.5. Metallurgical And Materials Transactions A-Physical Metallurgy And Materials Science, 31(7):1857-1865, 2000.

[21] P. Finkel, M. W. Barsoum, and T. El-Raghy. Low temperature dependencies of the elastic properties of Ti4AlN3, Ti3Al1.1C1.8, and Ti3SiC2. Journal Of Applied Physics, 87(4):1701-1703, 2000.

[22] F. C. Frank and A. N. Stroh. On the theory of kinking. Proceedings Of The Physical Society Of London Section B, 65(394):811-821, 1952.

[23] J. M. Molina-Aldareguia, J. Emmerlich, J. P. Palmquist, U. Jansson, and L. Hultman. Kink formation around indents in laminated Ti3SiC2 thin films studied in the nanoscale. Scripta Materialia, 49(2):155-160, 2003. 
[24] S. Myhra, J. W. B. Summers, and E. H. Kisi. Ti3SiC2 - A layered ceramic exhibiting ultra-low friction. Materials Letters, 39(1):6-11, 1999.

[25] A. Crossley, E. H. Kisi, J. W. B. Summers, and S. Myhra. Ultra-low friction for a layered carbide-derived ceramic, Ti3SiC2, investigated by lateral force microscopy (LFM). Journal Of Physics D-Applied Physics, 32(6):632-638, 1999.

[26] T. El-Raghy, A. Zavaliangos, M. W. Barsoum, and S. R. Kalidindi. Damage mechanisms around hardness indentations in Ti3SiC2. Journal of the American Ceramic Society, 80(2):513-516, 1997.

[27] M. W. Barsoum, T. ElRaghy, and L. U. J. T. Ogbuji. Oxidation of Ti3SiC2 in air. Journal Of The Electrochemical Society, 144(7):2508-2516, 1997.

[28] M. W. Barsoum, N. Tzenov, A. Procopio, T. El-Raghy, and M. Ali. Oxidation of Tin+1AlXn $(\mathrm{n}=1-3$ and $\mathrm{X}=\mathrm{C}, \mathrm{N})$ - II. Experimental results. Journal Of The Electrochemical Society, 148(8):C551-C562, 2001.

[29] L. Farber and M. W. Barsoum. Isothermal sections in the Cr-Ga-N system in the 650-1000 degrees C temperature range. Journal Of Materials Research, 14(6):2560$2566,1999$.

[30] R. Radhakrishnan, J. J. Williams, and M. Akinc. Synthesis and high-temperature stability of Ti3SiC2. Journal Of Alloys And Compounds, 285(1-2):85-88, 1999.

[31] N. Tzenov, M. W. Barsoum, and T. El-Raghy. Influence of small amounts of Fe and $\mathrm{V}$ on the synthesis and stability of Ti3SiC2. Journal Of The European Ceramic Society, 20(6):801-806, 2000.

[32] T. El-Raghy and M. W. Barsoum. Diffusion kinetics of the carburization and silicidation of Ti3SiC2. Journal Of Applied Physics, 83(1):112-119, 1998.

[33] W. Tian, P. Wang, G. Zhang, Y. Kan, Y. Li, and D. Yan. Synthesis and thermal and electrical properties of bulk Cr2AlC. Scripta Materialia, 54(5):841-846, 2006.

[34] Z.J. Lin, M.J. Zhuo, M.S. Li, J.Y. Wang, and Y.C. Zhou. Synthesis and microstructure of layered-ternary Ti2AlN ceramic. Scripta Materialia, 56(12):1115-1118, 2007.

[35] Z. F. Zhang, Z. M. Sun, H. Hashimoto, and T. Abe. Application of pulse discharge sintering (PDS) technique to rapid synthesis of Ti3SiC2 from Ti/Si/C powders. Journal of the European Ceramic Society, 22(16):2957-2961, 2002.

[36] H. Li, D. Chen, J. Zhou, J. H. Zhao, and L. H. He. Synthesis of Ti3SiC2 by pressureless sintering of the elemental powders in vacuum. Materials Letters, 58(11):17411744, April 2004. 
[37] J. P. Palmquist, U. Jansson, T. Seppanen, P. O. A. Persson, J. Birch, L. Hultman, and P. Isberg. Magnetron sputtered epitaxial single-phase Ti3SiC2 thin films. Applied Physics Letters, 81(5):835-837, 2002.

[38] J. Emmerlich, H. Hogberg, S. Sasvari, P. O. A. Persson, L. Hultman, J. P. Palmquist, U. Jansson, J. M. Molina-Aldareguia, and Z. Czigany. Growth of Ti3SiC2 thin films by elemental target magnetron sputtering. Journal of Applied Physics, 96(9):48174826, 2004.

[39] O. Wilhelmsson, J. P. Palmquist, T. Nyberg, and U. Jansson. Deposition of Ti2AlC and Ti3AlC2 epitaxial films by magnetron sputtering. Applied Physics Letters, 85(6):1066-1068, 2004.

[40] H. Hogberg, L. Hultman, J. Emmerlich, T. Joelsson, P. Eklund, J. M. MolinaAldareguia, J. P. Palmquist, O. Wilhelmsson, and U. Jansson. Growth and characterization of MAX-phase thin films. Surface and Coatings Technlogy, 193(1-3):6-10, 2005.

[41] T. Joelsson, A. Horling, J. Birch, and L. Hultman. Single-crystal Ti2AlN thin films. Applied Physics Letters, 86(11):111913, 2005.

[42] C. Walter, D. P. Sigumonrong, T. El-Raghy, and J. M. Schneider. Towards large area deposition of Cr2AlC on steel. Thin Solid Films, 515(2):389-393, 2006.

[43] M. Beckers, N. Schell, R. M. S. Martins, A. Mucklich, W. Moller, and L. Hultman. Microstructure and nonbasal-plane growth of epitaxial Ti2AlN thin films. Journal of Applied Physics, 99(3):034902, 2006.

[44] P. Eklund, M. Beckers, J. Frodelius, H. Hogberg, and L. Hultman. Magnetron sputtering of Ti3SiC2 thin films,from a compound target. Journal Of Vacuum Science \& Technology A, 25(5):1381-1388, 2007.

[45] J. Frodelius, M. Sonestedt, S. Björklund, J.P. Palmquist, K. Stiller, H. Högberg, and L. Hultman. Ti2AlC coatings deposited by High Velocity Oxy-Fuel spraying. Surface and Coatings Technology, 202(24):5976-5981, 2008.

[46] C. Lange, M. W. Barsoum, and P. Schaaf. Towards the synthesis of MAX-phase functional coatings by pulsed laser deposition. Applied Surface Science, 254(4):1232$1235,2007$.

[47] S. Acquaviva, E. D’Anna, L. Elia, M. Fernandez, G. Leggieri, A. Luches, M. Martino, P. Mengucci, and A. Zocco. Characterization of TiAlN films deposited by reactive pulsed laser ablation. Thin Solid Films, 379(1-2):45-49, 2000. 
[48] H. D. Lee and W. T. Petuskey. New ternary nitride in the Ti-Al-N system. Journal Of The American Ceramic Society, 80(3):604-608, 1997.

[49] M. W. Barsoum, L. Farber, I. Levin, A. Procopio, T. El-Raghy, and A. Berner. High-resolution transmission electron microscopy of Ti4AlN3, or Ti3Al2N2 revisited. Journal Of The American Ceramic Society, 82(9):2545-2547, 1999.

[50] A. T. Procopio, T. El-Raghy, and M. W. Barsoum. Synthesis of Ti4AlN3 and phase equilibria in the Ti-Al-N system. Metallurgical And Materials Transactions A-Physical Metallurgy And Materials Science, 31(2):373-378, 2000.

[51] J. J. Hu, J. E. Bultman, S. Patton, and J. S. Zabinski. Pulsed laser deposition and properties of M(n-1)AX(n) phase formulated Ti3SiC2 thin films. Tribology Letters, 16(1-2):113-122, 2004.

[52] P. Eklund, J. P. Palmquist, O. Wilhelmsson, U. Jansson, J. Emmerlich, H. Hogberg, and L. Hultman. Comment on "Pulsed laser deposition and properties of $\mathrm{M}(\mathrm{n}+1) \mathrm{AX}(\mathrm{x})$ phase formulated Ti3SiC2 thin films". Tribology Letters, 17(4):977978, 2004 .

[53] J. J. Hu and J. S. Zabinski. Comment on "Pulsed laser deposition and properties of $\mathrm{M}(\mathrm{n}+1) \mathrm{AX}(\mathrm{x})$ phase formulated Ti3SiC2 thin films" - Reply. Tribology Letters, 17(4):979-982, 2004.

[54] D. Bandyopadhyay. The Ti-Si-C system (Titanium-Silicon-Carbon). Journal Of Phase Equilibria And Diffusion, 25(5):415-420, 2004.

[55] J. P. Palmquist, S. Li, P. O. A. Persson, J. Emmerlich, O. Wilhelmsson, H. Hogberg, M. I. Katsnelson, B. Johansson, R. Ahuja, O. Eriksson, L. Hultman, and U. Jansson. $\mathrm{M}(\mathrm{n}+1) \mathrm{AX}(\mathrm{n})$ phases in the Ti-Si-C system studied by thin-film synthesis and ab initio calculations. Physical Review B, 70(16):165401, 2004.

[56] J. Emmerlich, D. Music, P. Eklund, O. Wilhelmsson, U. Jansson, J. M. Schneider, H. Hogberg, and L. Hultman. Thermal stability of Ti3SiC2 thin films. Acta Materialia, 55(4):1479-1488, 2007.

[57] P. Eklund, J. Emmerlich, H. Hogberg, O. Wilhelmsson, P. Isberg, J. Birch, R. O. A. Persson, U. Jansson, and L. Hultman. Structural, electrical, and mechanical properties of nc-TiC/a-SiC nanocomposite thin films. Journal Of Vacuum Science and Technology B, 23(6):2486-2495, 2005.

[58] S. Veprek. The search for novel, superhard materials. Journal Of Vacuum Science and Technology A, 17(5):2401-2420, 1999. 
[59] T.B. Massalski and H. Okamoto, editors. Binary Alloy Phase Diagrams. ASM International, 1990.

[60] K. Bewilogua, H. J. Heinitz, B. Rau, and S. Schulze. A chromium carbide phase with B1 structure in thin films prepared by ion plating. Thin Solid Films, 167(12):233-244, 1988 .

[61] J. M. Schneider, Z. Sun, R. Mertens, F. Uestel, and R. Ahuja. Ab initio calculations and experimental determination of the structure of Cr2AlC. Solid State Communications, 130(7):445-449, 2004.

[62] V. Raghavan. Al-C-Cr (Aluminum-Carbon-Chromium). Journal of Phase Equilibria and Diffusion, 29(1):49-50, 2008.

[63] B. Hallstedt, D. Music, and Z. Sun. Thermodynamic evaluation of the Al-Cr-C system. International Journal Of Materials Research, 97(5):539-542, 2006.

[64] Z. J. Lin, Y. C. Zhou, and M. S. Li. Synthesis, microstructure, and property of Cr2AlC. Journal Of Materials Science and Technology, 23(6):721-746, 2007.

[65] R. Mertens, Z. M. Sun, D. Music, and J. M. Schneider. Effect of the composition on the structure of $\mathrm{Cr}-\mathrm{Al}-\mathrm{C}$ investigated by combinatorial thin film synthesis and ab initio calculations. Advanced Engineering Materials, 6(11):903-907, 2004.

[66] N. Durlu, U. Gruber, M. A. Pietzka, H. Schmidt, and J. Schuster. Phases and phase equilibria in the quaternary system Ti-Cu-Al-N at 850 degrees C. Zeitschrift für Metallkunde, 88(5):390-400, 1997.

[67] M. W. Barsoum and J. C. Schuster. Comment on 'New ternary nitride in the Ti-Al-N system '. Journal Of The American Ceramic Society, 81(3):785-786, 1998.

[68] A. T. Procopio, M. W. Barsoum, and T. El-Raghy. Characterization of Ti4AlN3. Metallurgical and Materials Transactions A-Physical Metallurgy and Materials Science, 31(2):333-337, 2000.

[69] V. Raghavan. Al-N-Ti (Aluminum-Nitrogen-Titanium). Journal Of Phase Equilibria And Diffusion, 27(2):159-162, 2006.

[70] C. J. Rawn, M. W. Barsoum, T. El-Raghy, A. Procipio, C. M. Hoffmann, and C. R. Hubbard. Structure of Ti4AlN3 - a layered M(n+1)AX(n) nitride. Materials Research Bulletin, 35(11):1785-1796, 2000.

[71] W. S. Williams. Transition-metal carbides. Progress in Solid State Chemistry, 6:57-118, 1971. 
[72] P. O. A. Persson, S. Kodambaka, I. Petrov, and L. Hultman. Epitaxial Ti2AlN(0001) thin film deposition by dual-target reactive magnetron sputtering. Acta Materialia, 55(13):4401-4407, 2007.

[73] M. Beckers, N. Schell, R. M. S. Martins, A. Mucklich, and W. Moller. Phase stability of epitaxially grown Ti2AlN thin films. Applied Physics Letters, 89(7):074101, 2006.

[74] M. Beckers, N. Schell, R. M. S. Martins, A. Mucklich, W. Moller, and L. Hultman. Nucleation and growth of Ti2AlN thin films deposited by reactive magnetron sputtering onto MgO(111). Journal Of Applied Physics, 102(7):074916, 2007.

[75] F. Adibi, I. Petrov, L. Hultman, U. Wahlstrom, T. Shimizu, D. McIntyre, J. E. Greene, and J.-E. Sundgren. Defect structure and phase transitions in epitaxial metastable cubic Ti0.5Al0.5N alloys grown on $\mathrm{MgO}(001)$ by ultra-high-vacuum magnetron sputter deposition. Journal of Applied Physics, 69(9):6437-6450, 1991.

[76] C. Hoglund, M. Beckers, N. Schell, J. von Borany, J. Birch, and L. Hultman. Topotaxial growth of Ti2AlN by solid state reaction in $\mathrm{AlN} / \mathrm{Ti}(0001)$ multilayer thin films. Applied Physics Letters, 90(17):174106, 2007.

[77] D.B. Chrisey and G.K. Hubler, editors. Pulsed Laser Deposition of Thin Films. Wiley, New York, 1994.

[78] H. M. Smith and A. F. Turner. Vacuum Deposited Thin Films Using A Ruby Laser. Applied Optics, 4(1):147-148, 1965.

[79] D. Dijkkamp, T. Venkatesan, X. D. Wu, S. A. Shaheen, N. Jisrawi, Y. H. Minlee, W. L. Mclean, and M. Croft. Preparation Of Y-Ba-Cu Oxide Superconductor Thin-Films Using Pulsed Laser Evaporation From High-Tc Bulk Material. Applied Physics Letters, 51(8):619-621, 1987.

[80] K. H. Wu, C. L. Lee, J. Y. Juang, T. M. Uen, and Y. S. Gou. Insitu Growth Of Y1Ba2Cu3O7-X Superconducting Thin-Films Using A Pulsed Neodymium Yttrium-Aluminum-Garnet Laser With CO2-Laser Heated Substrates. Applied Physics Letters, 58(10):1089-1091, 1991.

[81] H. Schwarz and H. A. Tourtellotte. Vacuum Deposition By High-Energy Laser With Emphasis On Barium Titanate Films. Journal Of Vacuum Science and Technology, 6(3):373-378, 1969.

[82] K. H. Wong, S. K. Hau, P. W. Chan, L. K. Leung, C. L. Choy, and H. K. Wong. XeCl Excimer Laser Interaction With Partially-Stabilized Zirconia Target. Journal Of Materials Science Letters, 10(14):801-803, 1991. 
[83] S. Fähler, M. Stormer, and H. U. Krebs. Origin and avoidance of droplets during laser ablation of metals. Applied Surface Science, 110:433-436, 1997.

[84] D. Lubben, S. A. Barnett, K. Suzuki, S. Gorbatkin, and J. E. Greene. Laser-Induced Plasmas For Primary Ion Deposition Of Epitaxial Ge And Si Films. Journal Of Vacuum Science and Technology B, 3(4):968-974, 1985.

[85] H. Dupendant, J. P. Gavigan, D. Givord, A. Lienard, J. P. Rebouillat, and Y. Souche. Velocity Distribution Of Micron-Size Particles In Thin-Film Laser Ablation Deposition (LAD) Of Metals And Oxide Superconductors. Applied Surface Science, 43:369-376, 1989.

[86] A. Tselev, A. Gorbunov, and W. Pompe. Cross-beam pulsed laser deposition: General characteristic. Review of Scientific Instruments, 72(6):2665-2672, 2001.

[87] K. Murakami. Laser Ablation of Electronic Materials: Basic Mechanisms and Applications (European Materials Research Society Monographs, Vol. 4), pages 125-140. Elsevier, 1992.

[88] C. Doughty, A.T. Findikoglu, and T. Venkatesan. Steady state pulsed laser deposition target scanning for improved plume stability and reduced particle density. Applied Physics Letters, 66(10):1276-1278, 1995.

[89] S. Fähler and H. U. Krebs. Calculations and experiments of material removal and kinetic energy during pulsed laser ablation of metals. Applied Surface Science, 968:61-65, 1996.

[90] J. Perriere, E. Millon, W. Seiler, C. Boulmer-Leborgne, V. Craciun, O. Albert, J. C. Loulergue, and J. Etchepare. Comparison between ZnO films grown by femtosecond and nanosecond laser ablation. Journal Of Applied Physics, 91(2):690-696, 2002.

[91] R. Kelly and R.W. Dreyfus. Reconsidering the mechanisms of laser sputtering with Knudsen-layer formation taken into account. Nuclear Instruments and Methods in Physics Research Section B: Beam Interactions with Materials and Atoms, 32(14):341-348, 1988.

[92] J. C. S. Kools, T. S. Baller, S. T. Dezwart, and J. Dieleman. Gas-Flow Dynamics In Laser Ablation Deposition. Journal Of Applied Physics, 71(9):4547-4556, 1992.

[93] T. N. Hansen, J. Schou, and J. G. Lunney. Angle-resolved energy distributions of laser ablated silver ions in vacuum. Applied Physics Letters, 72(15):1829-1831, 1998. 
[94] G. Leggieri, A. Luches, M. Martino, A. Perrone, R. Alexandrescu, A. Barborica, E. Gyorgy, I. N. Mihailescu, G. Majni, and P. Mengucci. Laser reactive ablation deposition of silicon carbide films. Applied Surface Science, 96-8:866-869, 1996.

[95] H. Spillmann and P. R. Willmott. Growth of SiNx and SICx thin films by pulsed reactive crossed-beam laser ablation. Applied Physics A-Materials Science and Processing, 70(3):323-327, 2000.

[96] G. Leggieri, A. Luches, M. Martino, A. Perrone, G. Majni, P. Mengucci, and I. N. Mihailescu. Laser Reactive Ablation Deposition Of Titanium Carbide Films. Thin Solid Films, 258(1-2):40-45, 1995.

[97] T. Szorenyi, J. P. Stoquert, J. Perriere, F. Antoni, and E. Fogarassy. Correlation between hydrogen content and structure of pulsed laser deposited carbon nitride films. Diamond And Related Materials, 10(12):2107-2112, 2001.

[98] T. Szorenyi and E. Fogarassy. Pressure control of properties of pulsed laser deposited carbon and carbon nitride films. Journal Of Applied Physics, 94(3):2097-2101, 2003.

[99] M. L. De Giorgi, G. Leggieri, A. Luches, M. Martino, A. Perrone, A. Zocco, G. Barucca, G. Majni, E. Gyorgy, I. N. Mihailescu, and M. Popescu. Carbon nitride films deposited by reactive laser ablation. Applied Surface Science, 127:481-485, 1998.

[100] S. Acquaviva, E. D’Anna, M. L. De Giorgi, M. Fernandez, G. Leggieri, A. Luches, A. Zocco, and G. Majni. Carbon nitride films deposited by very high-fluence XeCl excimer-laser reactive ablation. Applied Surface Science, 154:369-375, 2000.

[101] M. L. De Giorgi, G. Leggieri, A. Luches, M. Martino, A. Perrone, G. Majni, P. Mengucci, J. Zemek, and I. N. Mihailescu. Laser-Reactive Ablation Deposition Of Silicon-Nitride Films. Applied Physics A-Materials Science $\&$ Processing, 60(3):275283, 1995.

[102] I. N. Mihailescu, E. Gyorgy, N. Chitica, V. S. Teodorescu, G. Mavin, A. Luches, A. Perrone, and M. Martino. A parametric study of the deposition of the TiN thin films by laser reactive ablation of titanium targets in nitrogen: The roles of the total gas pressure and the contaminations with oxides. Journal Of Materials Science, 31(11):2909-2915, 1996.

[103] R. Chowdhury, R. D. Vispute, K. Jagannadham, and J. Narayan. Characteristics of titanium nitride films grown by pulsed laser deposition. Journal Of Materials Research, 11(6):1458-1469, 1996. 
[104] H. D. Gu, K. M. Leung, C. Y. Chung, and X. D. Han. Room-temperature growth of high-purity titanium nitride by laser ablation of titanium in a nitrogen atmosphere. Surface andCoatings Technology, 110(3):153-157, 1998.

[105] A. G. Guidoni, A. Mele, T. M. DiPalma, C. Flamini, and S. Orlando. AlN thin film deposition by pulsed laser ablation of Al in NH3. Thin Solid Films, 295(1-2):77-82, 1997.

[106] J. M. Lackner, W. Waldhauser, R. Ebner, and R. J. Bakker. Chemistry and microstructure of PLD (Ti,Al)CxN1-x coatings deposited at room temperature. Applied Physics A-Materials Science and Processing, 79(4-6):1469-1471, 2004.

[107] D. Berling, A. Del Vecchio, S. Acquaviva, D. Bolmont, G. Leggieri, B. Loegel, M. L. De Giorgi, A. Luches, A. Mehdaoui, and L. Tapfer. Reactive laser deposition of high quality $\mathrm{YBaCuO}$ and $\mathrm{ErBaCuO}$ films. Applied Surface Science, 96-8:739-743, 1996.

[108] A. B. Kaul, T. D. Sands, and T. Van Duzer. High-T-c superconducting NbN films with low particulate density grown at 25 degrees $\mathrm{C}$ using pulsed laser deposition. Journal Of Materials Research, 16(5):1223-1226, 2001.

[109] G. Dinescu, E. Aldea, M. L. De Giorgi, A. Luches, A. Perrone, and A. Zocco. Optical emission spectroscopy of molecular species in plasma induced by laser ablation of carbon in nitrogen. Applied Surface Science, 127:697-702, 1998.

[110] J. Hermann, A. L. Thomann, C. Boulmer-Leborgne, B. Dubreuil, M. L. De Giorgi, A. Perrone, A. Luches, and I. N. Mihailescu. Plasma Diagnostics In Pulsed-Laser TiN Layer Deposition. Journal Of Applied Physics, 77(7):2928-2936, 1995.

[111] E. Carpene, M. Shinn, and P. Schaaf. Synthesis of highly oriented TiNx coatings by free-electron laser processing of titanium in nitrogen gas. Applied Physics AMaterials Science and Processing, 80(8):1707-1710, 2005.

[112] D. Höche, H. Schikora, H. Zutz, R. Queitsch, A. Emmel, and P. Schaaf. Microstructure of TiN coatings synthesized by direct pulsed Nd : YAG laser nitriding of titanium: Development of grain size, microstrain, and grain orientation. Applied Physics A-Materials Science and Processing, 91(2):305-314, 2008.

[113] V. S. Teodorescu, I. N. Mihailescu, E. Gyorgy, A. Luches, M. Martino, L. C. Nistor, J. van Landuyt, and J. Hermann. The study of a crater forming on the surface of a Ti target submitted to multipulse excimer laser irradiation under low pressure N2. Journal Of Modern Optics, 43(9):1773-1784, 1996. 
[114] B. Angleraud, M. Cahoreau, I. Jauberteau, J. Aubreton, and A. Catherinot. Nitrogen ion beam-assisted pulsed laser deposition of boron nitride films. Journal of Applied Physics, 83(6):3398-3403, 1998.

[115] J. P. Zhao, Z. Y. Chen, T. Yano, T. Ooie, and M. Yoneda. Irradiation effect of low energy nitrogen-ion beam during pulsed laser deposition process on the structural and bonding properties of carbon-nitride thin films. Journal Of Applied Physics, 89(3):1580-1587, 2001.

[116] R. Hühne, S. Fähler, and B. Holzapfel. Thin biaxially textured TiN films on amorphous substrates prepared by ion-beam assisted pulsed laser deposition. Applied Physics Letters, 85(14):2744-2746, 2004.

[117] R. Hühne, S. Fähler, L. Schultz, and B. Holzapfel. Thin biaxially textured MgO and TiN films prepared by ion-beam assisted pulsed laser deposition for coated conductor applications. Physica C-Superconductivity And Its Applications, 426:893-898, 2005.

[118] R. Hühne, K. Guth, M. Kidszun, R. Kaltofen, L. Schultz, and B. Holzapfel. Metallic seed layers for ion-beam assisted pulsed laser deposition of highly textured transition metal nitride films. Journal Of Physics D-Applied Physics, 41(24):245404, 2008.

[119] Z. M. Ren, Y. F. Lu, H. Q. Ni, T. Y. F. Liew, B. A. Cheong, S. K. Chow, M. L. $\mathrm{Ng}$, and J. P. Wang. Room temperature synthesis of c-AlN thin films by nitrogenion-assisted pulsed laser deposition. Journal Of Applied Physics, 88(12):7346-7350, 2000 .

[120] Y. F. Lu, Z. M. Ren, T. C. Chong, B. A. Cheong, S. K. Chow, and J. P. Wang. Ionassisted pulsed laser deposition of aluminum nitride thin films. Journal Of Applied Physics, 87(3):1540-1542, 2000.

[121] R. Hühne, C. Beyer, B. Holzapfel, C. G. Oertel, L. Schultz, and W. Skrotzki. Growth of biaxial textured MgO-Layers by ion-beam assisted pulsed laser deposition. Crystal Research And Technology, 35(4):419-425, 2000.

[122] R. Hühne, C. Beyer, B. Holzapfel, C. G. Oertel, L. Schultz, and W. Skrotzki. Formation and destruction of cube texture in $\mathrm{MgO}$ films using ion beam assisted pulsed laser deposition. Journal Of Applied Physics, 90(2):1035-1039, 2001.

[123] V. Betz, B. Holzapfel, and L. Schultz. In situ reflection high energy electron bombardment analysis of biaxially oriented yttria-stabilized zirconia thin film growth on amorphous substrates. Thin Solid Films, 301(1-2):28-34, 1997. 
[124] V. Betz, B. Holzapfel, D. Raouser, and L. Schultz. In-plane aligned Pr6O11 buffer layers by ion-beam assisted pulsed laser deposition on metal substrates. Applied Physics Letters, 71(20):2952-2954, 1997.

[125] S. Six, J. W. Gerlach, and B. Rauschenbach. Ion beam assisted pulsed laser deposition of epitaxial aluminum nitride thin films on sapphire substrates. Surface and Coatings Technology, 142:397-401, 2001.

[126] S. Six and B. Rauschenbach. Stress generation during ion beam-assisted pulsed laser deposition of thin AlN films. Thin Solid Films, 415(1-2):285-289, 2002.

[127] B. Roas, L. Schultz, and G. Saemannischenko. Anisotropy Of The Critical CurrentDensity In Epitaxial YBa2Cu3Ox Films. Physical Review Letters, 64(4):479-482, 1990.

[128] U. C. Oh and J. H. Je. Effects Of Strain-Energy On The Preferred Orientation Of TiN Thin-Films. Journal Of Applied Physics, 74(3):1692-1696, 1993.

[129] R. Hühne, S. Fähler, B. Holzapfel, C. G. Oertel, L. Schultz, and W. Skrotzki. Mechanism of texture formation in $\mathrm{MgO}$ buffer layers using ion-beam assisted laser deposition. Physica C-Superconductivity And Its Applications, 372:825-827, 2002.

[130] M. Birkholz. Thin Film Analysis by X-Ray Scattering. Wiley VCH, 2006.

[131] R. Payling. Glow Discharge Optical Emission Spectroscopy. John Wiley \& Sons, 1997.

[132] D. R. Penn. Electron mean-free-path calculations using a model dielectric function. Physical Review B, 35(2):482-486, 1987.

[133] W. C. Oliver and G. M. Pharr. Measurement of hardness and elastic modulus by instrumented indentation: Advances in understanding and refinements to methodology. Journal Of Materials Research, 19(1):3-20, 2004.

[134] S. Cusenza. Investigation of the Amorphization of iron and austenitic stainless steel films by supersaturation with Boron, Carbon, Nitrogen and Oxygen. PhD thesis, Georg-August-Universität Göttingen, 2008.

[135] A. Holz. Gepulste Laserdeposition von Eisen-Platin-Schichten. Diplomarbeit, GeorgAugust-Universität Göttingen, 2006.

[136] R. Eason, editor. Pulsed Laser Deposition of Thin Films, chapter Pulsed Laser Deposition of Metals, pages 363-382. Wiley-Interscience, 2007. 
[137] S. Fähler, K. Sturm, and H.-U. Krebs. Resputtering during the growth of pulsedlaser-deposited metallic films in vacuum and in an ambient gas. Applied Physics Letters, 75(24):3766-3768, 1999.

[138] D. E. Wolfe and J. Singh. Titanium carbide coatings deposited by reactive ion beamassisted, electron beam-physical vapor deposition. Surface and Coatings Technology, 124(2-3):142-153, 2000.

[139] A. Kumar, H. L. Chan, and J. S. Kapat. Deposition and characterization of titanium carbide coatings using laser ablation method. Applied Surface Science, 127-129:549$552,1998$.

[140] G. De Maria, D. Ferro, L. D’Alessio, R. Teghil, and S. M. Barinov. Hardness of titanium carbide films deposited on silicon by pulsed laser ablation. Journal of Materials Science, 36(4):929-935, 2001.

[141] E. O. Hall. The Deformation And Ageing Of Mild Steel .3. Discussion Of Results. Proceedings Of The Physical Society Of London Section B, 64(381):747-753, 1951.

[142] N. J. Petch. The Cleavage Strength Of Polycrystals. Journal Of The Iron And Steel Institute, 174(1):25-28, 1953.

[143] P. H. Mayrhofer, C. Mitterer, L. Hultman, and H. Clemens. Microstructural design of hard coatings. Progress In Materials Science, 51(8):1032-1114, 2006.

[144] H. Conrad and J. Narayan. On the grain size softening in nanocrystalline materials. Scripta Materialia, 42(11):1025-1030, 2000.

[145] C. E. Carlton and P. J. Ferreira. What is behind the inverse Hall-Petch effect in nanocrystalline materials? Acta Materialia, 55(11):3749-3756, 2007.

[146] P. H. Mayrhofer, G. Tischler, and C. Mitterer. Microstructure and mechanical/thermal properties of $\mathrm{Cr}-\mathrm{N}$ coatings deposited by reactive unbalanced magnetron sputtering. Surface \& Coatings Technology, 142:78-84, 2001.

[147] P. H. Mayrhofer, C. Mitterer, and J. Musil. Structure-property relationships in single- and dual-phase nanocrystalline hard coatings. Surface $\&$ Coatings Technology, 174:725-731, 2003.

[148] J. M. López, F. J. Gordillo-Vázquez, M. Fernández, J. M. Albella, D. Cáceres, and I. Vergara. Investigation of $\mathrm{TiC}$ thin films synthesised by low energy IBAD from electron evaporation of TiC powder. Applied Surface Science, 172(1-2):110-116, 2001.

[149] R. W. M. Kwok. XPS Peak 4.1 for Windows 95/98, 2000. 
[150] C. D. Wagner, A. V. Naumkin, A. Kraut-Vass, J. W. Allison, C. J. Powell, and J. R. Rumble Jr. NIST X-ray Photoelectron Spectroscopy Database (NIST Standard Reference Database 20, Version 3.5), Online accessed: Feb 19, 2009, National Institute of Standards and Technology, Gaithersburg, MD. .

[151] K. Hirota, K. Mitani, M. Yoshinaka, and O. Yamaguchi. Simultaneous synthesis and consolidation of chromium carbides ( $\mathrm{Cr} 3 \mathrm{C} 2, \mathrm{Cr} 7 \mathrm{C} 3$ and $\mathrm{Cr} 23 \mathrm{C} 6)$ by pulsed electric-current pressure sintering. Materials Science And Engineering A-Structural Materials Properties Microstructure And Processing, 399(1-2):154-160, 2005.

[152] N. Marechal, E. Quesnel, and Y. Pauleau. Deposition Process And Characterization Of Chromium-Carbon Coatings Produced By Direct Sputtering Of A Magnetron Chromium Carbide Target. Journal of Materials Research, 9(7):1820-1828, 1994.

[153] Y. N. Kok, P. E. Hovsepian, Q. Luo, D. B. Lewis, J. G. Wen, and I. Petrov. Influence of the bias voltage on the structure and the tribological performance of nanoscale multilayer C/Cr PVD coatings. Thin Solid Films, 475(1-2):219-226, 2005.

[154] J. M. Schneider, D. P. Sigumonrong, D. Music, C. Walter, J. Emmerlich, R. Iskandar, and J. Mayer. Elastic properties of Cr2AlC by nanoindentation and ab initio thin films probed molecular dynamics. Scripta Materialia, 57(12):1137-1140, 2007.

[155] J. D. Hettinger, S. E. Lofland, P. Finkel, T. Meehan, J. Palma, K. Harrell, S. Gupta, A. Ganguly, T. El-Raghy, and M. W. Barsoum. Electrical transport, thermal transport, and elastic properties of $\mathrm{M} 2 \mathrm{AlC}(\mathrm{M}=\mathrm{Ti}, \mathrm{Cr}, \mathrm{Nb}$, and $\mathrm{V})$. Physical Review B, $72(11): 115120,2005$.

[156] S. Schwarz, Y. Musayev, S. M. Rosiwal, C. Schaufler, R. F. Singer, and H. Meerkamm. High temperature diffusion chromizing as a successful method for CVDdiamond coating of steel. Diamond and Related Materials, 11(3-6):757-762, 2002.

[157] E. Friemann and F. Sauerwald. Über Mehrstoffsysteme mit Eisen. IV. Das System Cr-C (und Fe-Cr-C). Zeitschrift für anorganische und allgemeine Chemie, 203(1):64-74, 1931.

[158] F. Sauerwald, W. Teske, and G. Lempert. Über Mehrstoffsysteme mit Eisen. V. Röntgenographische Ergänzungen über die Systeme Cr-C und Fe-Si-P. Zeitschrift für anorganische und allgemeine Chemie, 210(1):21-25, 1933.

[159] P. H. Mayrhofer, A. Horling, L. Karlsson, J. Sjolen, T. Larsson, C. Mitterer, and L. Hultman. Self-organized nanostructures in the Ti-Al-N system. Applied Physics Letters, 83(10):2049-2051, 2003. 
[160] H. Yang and P. G. McCormick. Synthesis of titanium oxynitride by mechanical milling. Journal of Materials Science, 28(20):5663-5667, 1993. 


\section{Publikationen}

P. Schaaf , C. Lange, A. Holz, M. Weisheit, S.Fähler and K. Zhang. Structure and anisotropy of epitaxial fcc FePt films. Applied Surface Science, 253(19):8107-8110, 2007.

C. Lange, M.W. Barsoum and P. Schaaf. Towards the synthesis of MAX-phase functional coatings by pulsed laser deposition. Applied Surface Science, 254(4):1232-1235, 2007.

P. Schaaf, H. Schikora, D. Höche, C. Lange, V. Drescher and J. Wilden. Laser clad surfaces for shark-skin effect by high-temperature activation. Surface $\&$ Coatings Technology, 203(5-7):470-475, 2008.

C. Lange, J. Schawohl, M. Wilke, M.W. Barsoum and P. Schaaf. Ion beam-assisted pulsed laser deposition from a $\mathrm{Ti}_{3} \mathrm{SiC}_{2} \mathrm{MAX}$-Phase target. Surface $\&$ Coatings Technology, submitted.

C. Lange, J. Schawohl, M. Wilke, M.W. Barsoum and P. Schaaf. Ion beam-assisted pulsed laser deposition from a $\mathrm{Cr}_{2} \mathrm{AlC}$ MAX-Phase target. In preparation. 


\section{Dank}

Die hier vorgelegte Arbeit wurde im Zeitraum von Februar 2005 bis zum April 2009 am II. Physikalischen Institut der Georg-August-Universität Göttingen angefertigt. Ohne die Unterstützung zahlreicher Menschen wäre sie nicht zustande gekommen.

Mein Dank gilt deswegen zuallererst Herrn Prof. Dr. Peter Schaaf. Zum einen für die Möglichkeit, dieses Thema zur Promotion zu bearbeiten und den großen Freiraum, den er mir dabei ließ. Zum anderen für wertvolle Denkanstöße und einen geradezu unerschütterlichen Optimismus, der mich die schwierigen Phasen dieser Arbeit durchstehen ließ. Außerdem danke ich Herrn Prof. Dr. Hans-Ulrich Krebs für die Übernahme des Korreferats.

Der Aufbau der PLD-Anlage wäre ohne die Hilfe der im Haus ansässigen Werkstätten nicht möglich gewesen. Deswegen bedanke ich mich besonders bei der Feinmechanikwerkstatt und den E-Werkstätten des II. Physikalischen Instituts, sowie der Zentralwerkstatt der Fakultät für Physik.

Allen aktuellen und ehemaligen MitarbeiterInnen des II. Physikalischen Instituts in Göttingen danke ich für die sehr freundliche und angenehme Arbeitsatmosphäre, sowie für all die großen und kleinen Hilfestellungen in Sachen Wissenschaft und Verwaltung, während meiner Zeit hier im Hause. Besonders hervorheben möchte ich dabei Daniel Höche, Andreas König und Velimir Milinović.

Das Gleiche gilt auch für die MitarbeiterInnen des Fachgebietes Werkstoffe der Elektrotechnik und des Zentrums für Mikro- und Nanotechnologien an der TU Ilmenau. Vielen Dank vor allem an Marcus Wilke, Jens Schawohl, Elvira Remdt und Thomas Kups für all die Hilfe und Unterstützung während der dicht gepackten Messwochen, die ich in Ilmenau verbracht habe.

Dank und Respekt gelten außerdem: Mara Kastein, Daniel Kasten, Melanie Baier, Wiebke Harms, Silke Hampel, Hendrik Schikora, Catharina Wille, Henrike Wagner und Anne Uthoff. 


\section{Lebenslauf}

$\begin{array}{ll}\text { Name: } & \text { Christian Lange } \\ \text { Geburtstag: } & \text { 22. September } 1978 \\ \text { Geburtsort: } & \text { Dresden, Deutschland } \\ \text { Staatsangehörigkeit: } & \text { deutsch } \\ \text { Familienstand: } & \text { ledig }\end{array}$

\section{Ausbildung}

1985-1987

6. POS „Otto Grotewohl“, Dresden

1987-1991

3. POS „Herbert Bochow“ mit erweitertem Russischunterricht, Dresden

1991-1997

Spezialschule mathematisch-naturwissenschaftlich technischer Richtung „Martin Andersen Nexö“, Dresden;

1995 umbenannt in 12. Gymnasium Dresden-Blasewitz, Abschluß Abitur

09/1997 - 09/1998 Zivildienst

ab $10 / 1998$

Studium der Chemie an der Technischen Universität Dresden

$08 / 2003$

Diplom am Institut für Biochemie der TU Dresden

10/2003 - 12/2004 Wissenschaftlicher Mitarbeiter am Institut für Physikalische Chemie der TU Dresden

02/2005 - 09/2008 Wissenschaftlicher Mitarbeiter am II. Physikalischen Institut der Georg-August-Universität Göttingen

seit 10/2008 Wissenschaftlicher Mitarbeiter am Institut für Werkstofftechnik der Technischen Universität Ilmenau 